\title{
APLICAÇÃO DO MÉTODO DOS ELEMENTOS DE CONTORNO À ANÁLISE DE PAVIMENTOS DE EDIFÍCIOS
}

Tese apresentada à Escola de Engenharia de São Carlos, da Universidade de São Paulo, como parte dos requisitos para obtenção do Título de Doutor em Engenharia de Estruturas.

ORIENTADOR: Prof. Dr. Wilson Sérgio Venturini 
À minha amada esposa Júlia Elisa e ao meu filho Gabriel dedico, com carinho, este trabalho. A estes, minha gratidão e apreço. 


\section{AGRADECIMENTOS}

“ Assim diz o SENHOR: não se glorie o sábio na sua sabedoria, nem o forte na sua força, nem o rico nas suas riquezas; mas o que se gloriar, glorie-se nisto: em me conhecer e saber que eu sou o SENHOR, e faço misericórdia, juizo e justiça na terra; porque destas cousas me agrado, diz o SENHOR.” (Jr. 9:23, 24)

A Deus, que tudo criou, a Cristo Jesus, que todas as coisas sustenta; autor e consumador de toda ciência e sabedoria humanas, rendo minha gratidão e louvor por mais esta vitória concedida.

Ao Prof. Dr. Wilson Sérgio Venturini que, com tanta naturalidade e segurança, soube tão bem orientar todas as etapas deste trabalho.

À Universidade Federal de Goiás; À CAPES, através do programa PICDT, pelo apoio financeiro.

Aos professores e funcionários do Departamento de Estruturas da EESC-USP que colaboraram, de forma direta e indireta, a fim de que este trabalho pudesse ser concretizado. À Rosi Aparecida Jordão Rodrigues, à Maria Nadir Minatel pela atenção e apoio a mim dispensados. A Francisco Carlos Guete de Brito pelo trabalho paciente na confecção dos desenhos.

Aos amigos, irmãos em Cristo e familiares, pelo sustento e apoio fundamentais. 


\section{SUMÁRIO}

LISTA DE FIGURAS..........................................................................................

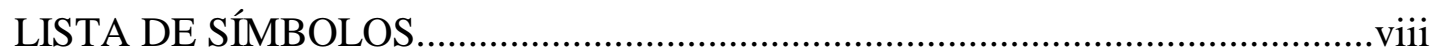

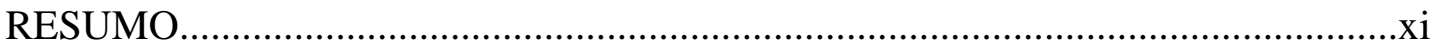

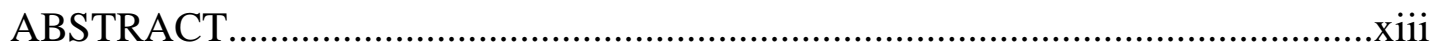

1. INTRODUÇÃO

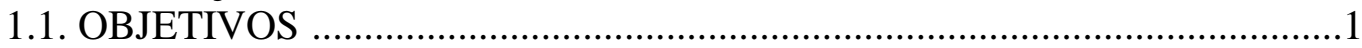

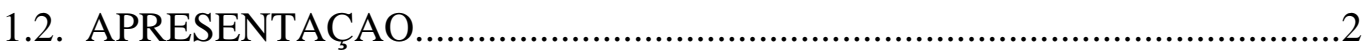

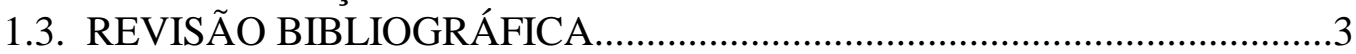

2. O MÉTODO DOS ELEMENTOS DE CONTORNO APLICADO ÀS PLACAS DE REISSNER

2.1. INTRODUÇÃO AO MÉTODO DOS ELEMENTOS DE CONTORNO............13

2.1.1. Equações de Navier ........................................................................18

2.1.2. Solução fundamental.........................................................................18

2.1.3. Teorema da Reciprocidade de Betti..................................................20

2.1.4. Identidade Somigliana..........................................................................

2.2.TEORIA DE PLACAS DE REISSNER_............................................................22

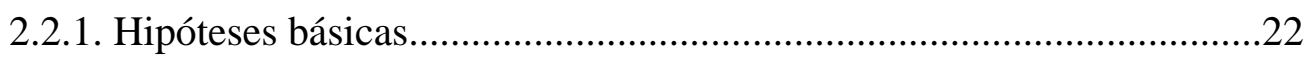

2.2.2. Sistema de Equações de Reissner..........................................................22

2.2.3. Condições de contorno...........................................................................24

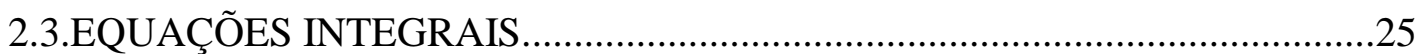

2.3.1. Equações integrais para deslocamentos em pontos do domínio...........25

2.3.2. Equações integrais para deslocamentos em pontos do contorno...........26

2.3.3. Equações integrais para esforços nos pontos do domínio.......................27

2.3.4. Transformação das Integrais das cargas e momentos distribuídos no Domínio em Integrais de Contorno ................................................28

2.3.5. Influência do Campo de Momentos Iniciais no Cálculo dos Esforços nos pontos do Domínio...............................................................32

2.3.6. Influência de cargas concentradas.............................................................33

2.4.DISCRETIZAÇÃO DAS EQUAÇÕES INTEGRAIS.........................................34

2.4.1. Considerações preliminares.................................................................34

2.4.2. Deslocamentos nos pontos do contorno....................................................

2.4.3. Deslocamentos nos pontos do domínio...................................................40

2.4.4. Esforços nos pontos do domínio.........................................................42

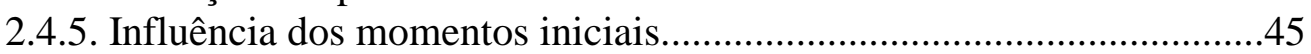

2.4.6. Técnica de solução.................................................................................50

3. ANÁLISE DE PLACAS ENRIJECIDAS

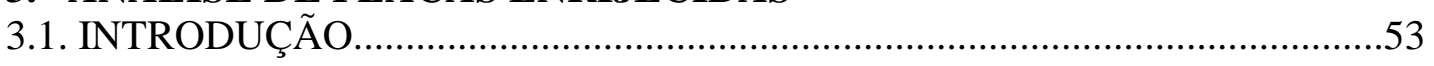

3.2. APOIOS EM LINHAS................................................................................53 


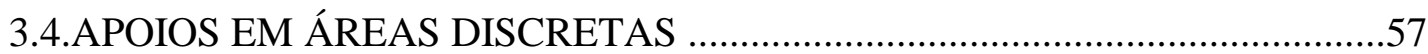

\section{ANÁLISE NÃO-LINEAR}

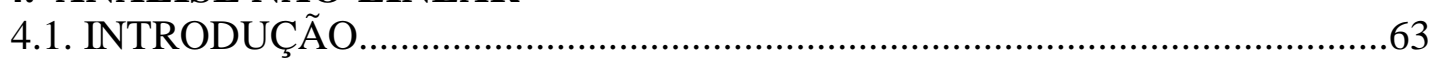

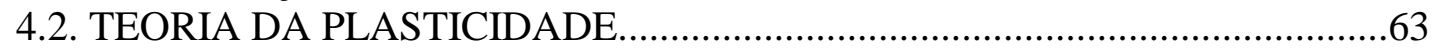

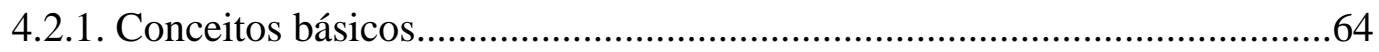

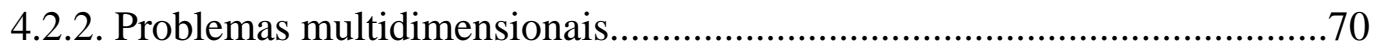

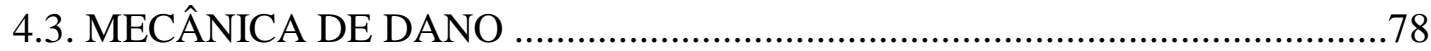

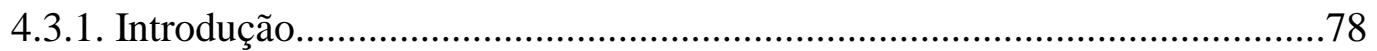

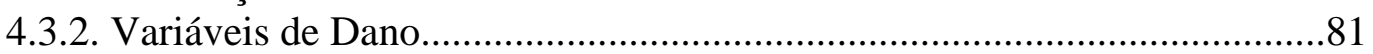

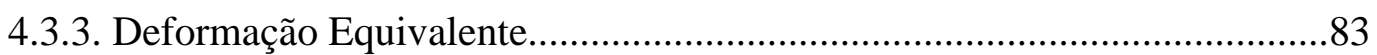

4.3.4. Modelo Constitutivo de Mazars.................................................................8 84

\section{SOLUÇÃO DO PROBLEMA NÃO LINEAR}

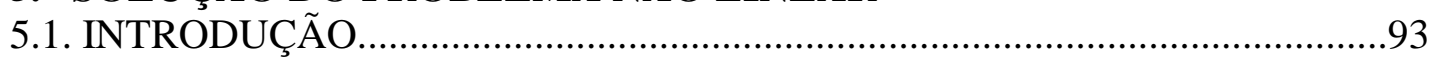

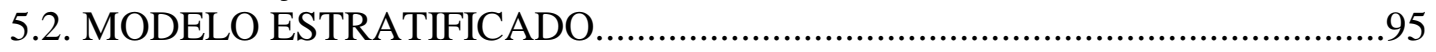

5.3. PROCEDIMENTO PARA ANULAR O ESFORÇO NORMAL.......................97

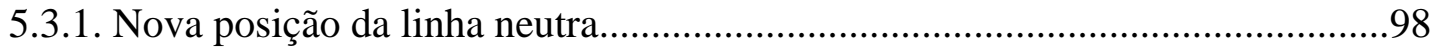

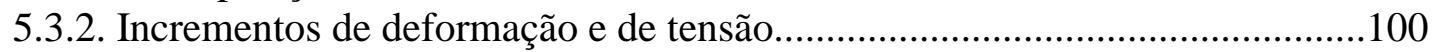

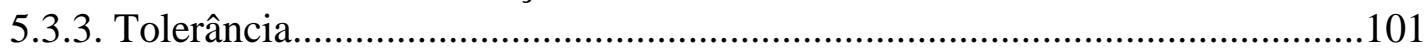

5.4.CONSIDERAÇÃO DAS TENSÕES TANGENCIAIS....................................101

5.5. ABORDAGEM DO PROCESSO DE RESOLUÇÃO …..............................102

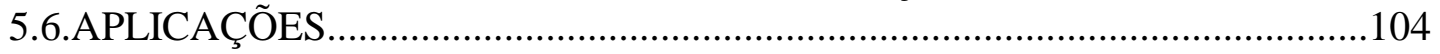

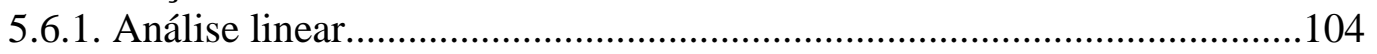

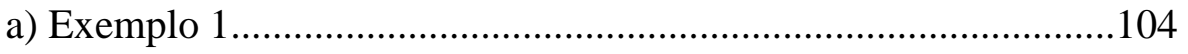

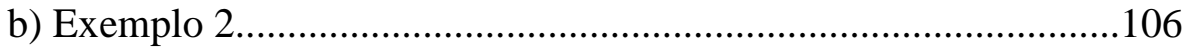

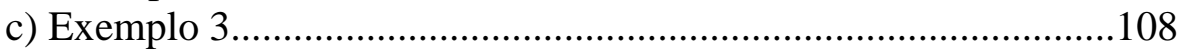

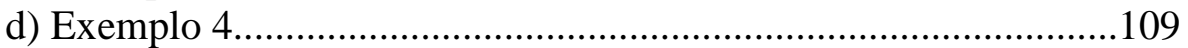

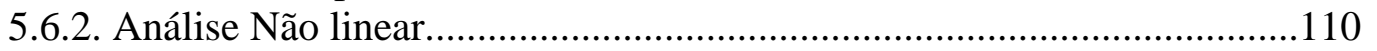

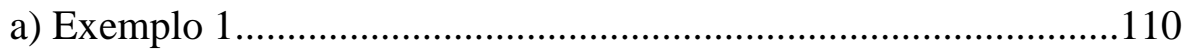

b) Exemplo 2 ...................................................................... 113

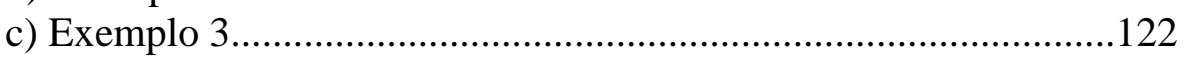

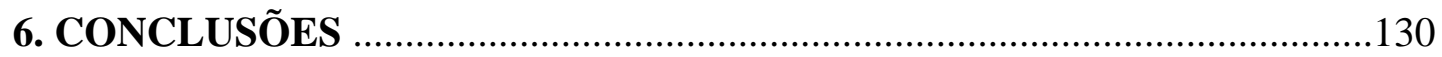

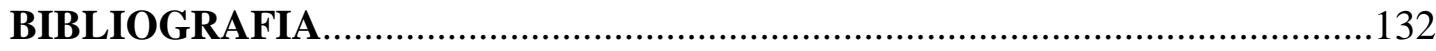

\section{APÊNDICES:}

APÊNDICE A: SOLUÇÕES FUNDAMENTAIS.................................................138

APÊNDICE B: FUNDAMENTOS MATEMÁTICOS ...........................................143

APÊNDICE C: FUNÇÕES DE BESSEL MODIFICADAS $\mathbf{K}_{\mathbf{0}}$ e $\mathbf{K}_{\mathbf{1}} \ldots \ldots \ldots \ldots \ldots \ldots \ldots . . . . . . . . . .154$

APÊNDICE D: FLUXOGRAMA DO PROGRAMA PRINCIPAL ........................157 


\section{LISTA DE FIGURAS}

FIGURA 2.1. Métodos numéricos aplicados aos problemas de Engenharia...............14

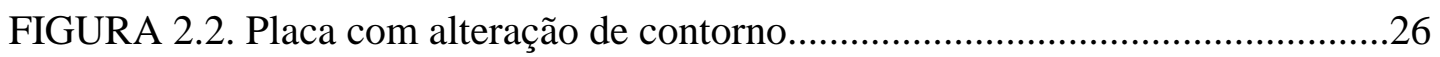

FIGURA 2.3. Cargas ou momentos distribuídos sobre domínios estreitos e

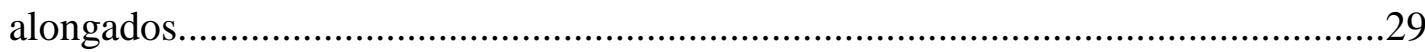

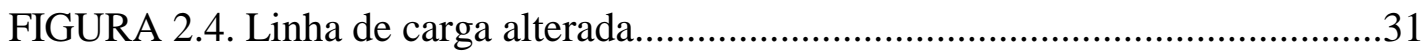

FIGURA 2.5. Elemento de contorno $\Gamma_{\mathrm{j}}$ e aproximação das variáveis do contorno....34

FIGURA 2.6. Pontos fonte $\xi$ colocados fora do domínio da placa.............................38

FIGURA 2.7. Divisão do domínio $\Omega$ em células triangulares....................................45

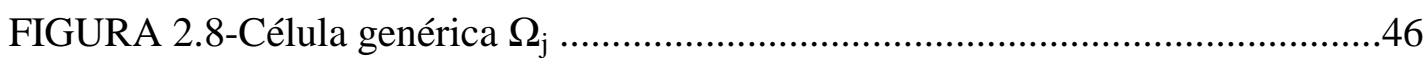

FIGURA 2.9. Subdivisão da célula com ponto singular............................................49

FIGURA 3.1. Associação placa - pilar .......................................................................

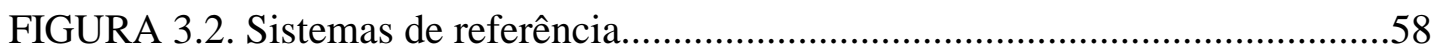

FIGURA 3.3. posições inicial e deformada do conjunto placa-pilar..........................59

FIGURA 3.4. Domínio interno carregado com $\sigma(\mathrm{X})$.................................................60

FIGURA 4.1. Diagramas tensão - deformação .....................................................64

FIGURA 4.2. Ensaio uniaxial: níveis de carregamento, descarregamento

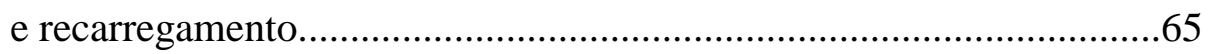

FIGURA 4.3. Modelos idealizados de comportamento..............................................66

FIGURA 4.4. Módulo tangente e módulo plástico...................................................67

FIGURA 4.5. Regras de endurecimento..............................................................69

FIGURA 4.6. Elemento de volume danificado.........................................................82

FIGURA 4.7. Hipótese de deformação equivalente..................................................84

FIGURA 4.8. Diagrama $\sigma \times \varepsilon$ - teste uniaxial de tração: representação de $\varepsilon_{\mathrm{do}} \ldots \ldots . . . . .87$

FIGURA 4.9. Superfície de ruptura no espaço das deformações principais...............88

FIGURA 4.10. Superfícies de ruptura no espaço das tensões principais

$$
\sigma_{1} \text { e } \sigma_{2}
$$

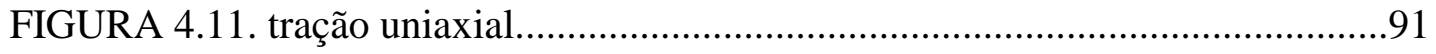

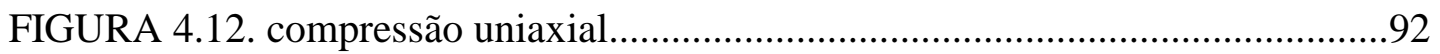

FIGURA 5.1. Distribuição de tensões em uma seção da placa....................................95

FIGURA 5.2. Modelo estratificado para o concreto armado......................................96 
FIGURA 5.3. Estimativa da nova posição da L.N....................................................98

FIGURA 5.4. Distribuição das deformações ao longo da seção transversal..............99

FIGURA 5.5. Incrementos de deformações e deformações totais............................100

FIGURA 5.6. Placa retangular simplesmente apoiada nas bordas

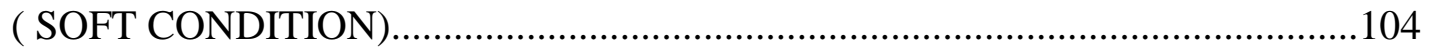

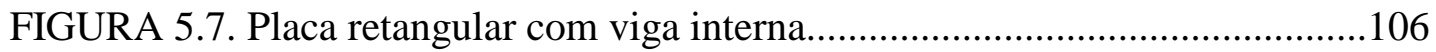

FIGURA 5.8. Placa quadrada engastada nos quatro lados.....................................108

FIGURA 5.9. Placa quadrada sobre quatro vigas elásticas....................................109

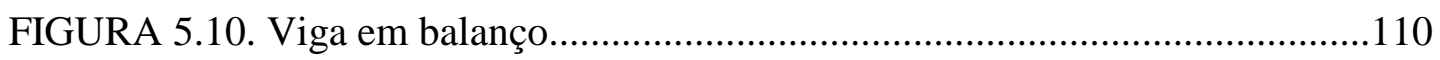

FIGURA 5.11. Curvas carga-deslocamento para os pontos internos.......................111

FIGURA 5.12. Curvas tensão - Deformação para o aço...........................................113

FIGURA 5.13. Curvas Cargas - Deslocamentos 1................................................115

FIGURA 5.14. Curvas carga - deslocamentos 2..................................................117

FIGURA 5.15. Evolução do dano nos pontos de Gauss Ex. 2.................................119

FIGURA 5.16. Curvas Carga - Deformação na armadura.....................................121

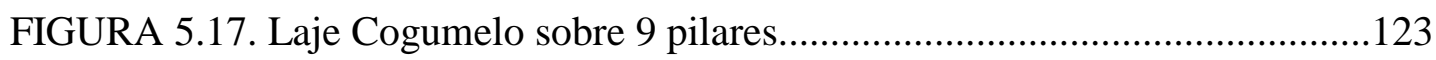

FIGURA 5.18. Curvas Incrementos - Momentos..................................................124

FIGURA 5.19. Evolução do dano nos pontos de Gauss - ex. 3 .................................125

FIGURA 5.20. Curvas incremento - Deformação nas armaduras ..........................126

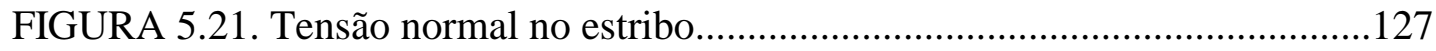

FIGURA 5.22. Absorção do esforço cortante (dir. x)................................................128

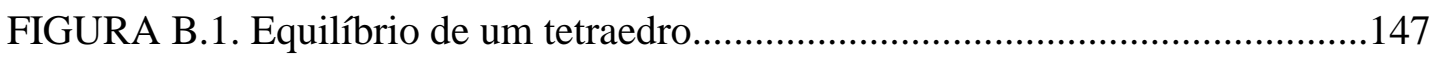

FIGURA B.2. Definição da função Delta de Dirac. Função $F(x, d, a)$.......................148

FIGURA B.3. Definiç̧ão de uma integral de contorno............................................150

FIGURA B.4. curva genérica para definição da condição de Hölder........................152 


\section{LISTA DE SÍMBOLOS}

\section{GREGOS}

$\alpha \quad$ Índice representativo das direções 1 e 2; coeficiente que determina a distância do ponto fonte ao contorno;

$\boldsymbol{\beta} \quad$ Índice representativo das direções 1 e 2;

$\boldsymbol{\beta}_{\mathbf{n}} \quad$ Fator de carga;

$\boldsymbol{\delta}_{\mathrm{ij}} \quad$ Delta de Kronecker;

$\boldsymbol{\varepsilon} \quad$ Raio de um contorno circular;

$\varepsilon_{\mathrm{ij}} \quad$ Componentes de deformação;

$\phi_{1}, \phi_{2} \quad$ Rotações nos planos X-Z e y-z, respectivamente;

$\boldsymbol{\gamma} \quad$ Distorção;

$\bar{\kappa}_{\mathbf{i}}(\varsigma) \quad$ Funções interpoladoras quadráticas;

$\lambda \quad$ Constante característica das equações de Reissner;

v Coeficiente de Poisson;

$\boldsymbol{\theta} \quad$ Ângulo que define a direção de $\mathbf{r}$;

$\rho \quad$ Densidade volumétrica;

$\boldsymbol{\sigma}(\mathbf{x}) \quad$ Tensão na área de interface placa-pilar;

$\sigma_{\mathrm{ij}} \quad$ Componentes de tensão;

$\xi \quad$ Ponto fonte;

$\zeta \quad$ Coordenadas locais homogêneas;

$\Omega \quad$ Domínio da placa; volume

$\boldsymbol{\Omega}_{\mathbf{q}}, \boldsymbol{\Omega}_{\mathbf{m}} \quad$ Subdomínios carregados com a carga $\mathbf{q}(\mathbf{x})$ e momento $\mathbf{m}_{\boldsymbol{\alpha}}(\mathbf{x})$, respectivamente;

$\Gamma \quad$ Contorno da placa; área de superfície;

$\Gamma_{\mathbf{q}}, \Gamma_{\mathrm{m}} \quad$ Contornos dos subdomínios $\boldsymbol{\Omega}_{\mathbf{q}}$, e $\boldsymbol{\Omega}_{\mathrm{m}}$, respectivamente;

$\Psi \quad$ Energia interna por unidade de volume;

$\Psi_{\mathbf{3 \theta}} \quad$ Componentes de deformação por esforço cortante; 
$\underset{\Psi}{\Psi} \quad$ Matriz que contém as coordenadas homogêneas de um ponto sobre uma célula;

ROMANOS:

- MAIÚSCULOS

B Vetor que contém os termos independentes;

$\mathbf{C}_{\mathbf{i j k l}} \quad$ Tensor de quarta ordem que contém as constantes elásticas;

D Rigidez da placa à flexão; variável que caracteriza o estado de dano local;

E Módulo de elasticidade longitudinal;

$\overline{\mathbf{E}} \quad$ Módulo de elasticidade do material danificado;

$\mathbf{E}_{\mathbf{t}} \quad$ Módulo tangente;

G Módulo de elasticidade transversal;

G, H Matrizes de influência dos valores nodais;

$\mathbf{I}_{\mathbf{x x}}, \mathbf{I}_{\mathbf{y y}}$ Momentos de inércia em relação aos eixos $\mathbf{x}$ e $\mathbf{y}$, respectivamente;

J Jacobiano de transformação das variáveis;

L Comprimento do elemento de contorno;

$\mathbf{M}_{\alpha \beta}^{\mathbf{o}} \quad$ Campo de momentos iniciais;

$\mathbf{M}_{\alpha \beta} \quad$ Momentos fletores e volventes por unidade de comprimento;

$\mathbf{P} \quad$ Carga concentrada aplicada à placa;

Q Potencial plástico;

$\mathbf{Q}_{3 \boldsymbol{\alpha}} \quad$ Forças cortantes por unidade de comprimento;

$\mathbf{R} \quad$ Ações do pilar sobre a placa;

S Área da interface placa-pilar; Entropia específica;

T Temperatura absoluta;

U Energia interna;

$\mathbf{W}_{\mathbf{p}} \quad$ trabalho plástico; 


\section{MINÚSCULOS:}

a Vetor de fluxo; vetor aceleração;

b largura de um domínio estreito e alongado;

$\mathbf{b}_{\mathbf{i}} \quad$ forças de volume;

d Distância do ponto fonte ao contorno;

d $\lambda$ Multiplicador plástico;

$\mathbf{f}\left(\sigma_{i j}\right)$ função do estado de tensão atual;

$\mathbf{f}_{\mathbf{i}} \quad$ forças de massa

$\mathbf{g}_{\mathbf{i j}} \quad$ Componentes da matriz $\mathbf{G}$;

h Espessura da placa;

$\mathbf{h}_{\mathrm{ij}} \quad$ Componentes da matriz $\mathbf{H}$;

h Fluxo de calor por unidade de área;

k Parâmetro de endurecimento;

k Matriz de rigidez do elemento;

$\mathbf{m}_{\boldsymbol{\alpha}} \quad$ Momento distribuído aplicado à placa;

$\mathbf{n}_{\boldsymbol{\beta}}$ Componentes da normal ao contorno;

$\mathbf{p}_{\mathbf{i j}} \quad$ Forças de superfície generalizadas;

q Carga transversal aplicada à placa;

r Distância entre o ponto fonte e o ponto campo;

u Energia interna específica;

$\mathbf{u}_{\mathbf{i}} \quad$ Componentes de deslocamento;

V Vetor velocidade;

W Deslocamento transversal da placa;

$\mathbf{X} \quad$ Ponto campo; 


\section{RESUMO}

BACARJI, E. Aplicação do método dos elementos de contorno à análise de pavimentos de edifícios. São Carlos, 2001. Tese (Doutorado) - Escola de engenharia de São Carlos - USP.

Neste trabalho utiliza-se uma formulação do Método dos Elementos de Contorno (MEC) para a análise de pavimentos de edifícios, dando-se particular ênfase à análise de lajes cogumelo feita com a incorporação da não-linearidade física. Nesta formulação são consideradas as tensões normais e cisalhantes possibilitando, assim, a determinação da resistência última da estrutura.

A formulação é inicialmente desenvolvida para a análise de flexão de placas utilizando-se a teoria de Reissner. A seguir, a formulação é estendida de modo a considerar a interação da placa com outros elementos, como vigas e pilares. $\mathrm{Na}$ interação placa-viga, o enrijecimento produzido é computado através de uma combinação com o método dos elementos finitos. Este modelo permite uma avaliação precisa dos momentos e forças cortantes nas interfaces da placa com os elementos lineares.

Admite-se a ocorrência de um campo de momentos iniciais, viabilizando, dentre outros, o estudo de pavimentos com não-linearidade física.

Para a análise do comportamento não-linear, implementa-se um algoritmo incremental-iterativo baseado no método da rigidez inicial.

Visando-se obter uma melhor representação do comportamento do concreto armado, a integração das tensões ao longo da espessura é feita por um esquema numérico tipo gaussiano; a contribuição da armadura é feita de modo discreto considerada concentrada em seu centro geométrico. Pode-se, assim, avaliar separadamente o processo de danificação do concreto e o escoamento das armaduras. Para o concreto adota-se o modelo de dano de Mazars e para as armaduras longitudinais, um modelo elastoplástico uniaxial com endurecimento isotrópico.

Quanto à absorção dos esforços oriundos das tensões cisalhantes, adota-se um modelo semelhante à idealização da treliça clássica de Ritter e Mörsch para vigas de concreto armado. Admite-se ainda que, após o início da fissuração, as tensões 
cisalhantes sejam absorvidas apenas pelas armaduras transversais. Para estas, adotase comportamento elástico linear.

Objetivando-se a comprovação da eficiência da formulação proposta, são analisados alguns exemplos cujos resultados são comparados com resultados experimentais ou resultados de outros métodos de análise.

Palavras chave: elementos de contorno, não-linearidade, análise de pavimentos. 


\section{ABSTRACT}

BACARJI, E. Application of the boundary element method to slab floor analysis. São Carlos, 2001. Tese (Doutorado) - Escola de engenharia de São Carlos - USP.

This work deals with a formulation of the boundary element method applied to slab floor analysis with special emphasis concrete flat slabs exhibiting physical non-linearities. In this formulation normal and shear components of the stress tensor are taken into account to capture more accurately the ultimate strength of the structural element.

The boundary element formulation in the context of Reissner's plate bending theory is initially studied. Then, the formulation is extended to deal with combinations of plate elements with other elements such as beams and columns and also to incorporate internal support effects, for which full contact is assumed over small areas. The plate-beam and plate-column interaction model is based on a combination with the finite element method. Thus, this model allows an accurate evaluation of the internal forces along the plate-linear element interfaces and also over its vicinity.

The presence of possible initial moment fields is also taken into account, which enables us to consider physical non-linear behaviours. The solution of the nonlinear system of algebraic equations is based on an iterative algorithm with constant matrix.

In order to obtain a better modelling of the reinforced concrete slabs, the stress integrals along the thickness are performed with an appropriate gauss scheme; the reinforcement contribution is computed by considering concentrated effects at its geometric centre. Thus, the concrete degradation and the steel yielding can be independently evaluated.

To represent the concrete behaviour the Mazars' damage model has been adopted, while the steel material is governed by a uniaxial elastoplastic criterion with isotropic hardening. After the initial cracking of the concrete the shear stresses are properly transferred to the shear reinforcement using the Mörsch truss concept. 
The accuracy of the proposed formulation is illustrated by the analysing some practical examples. The results obtained are compared with experimental results and other numerical technique solutions.

Key words: boundary elements, physical nonlinearities, and concrete slab floor. 


\section{CAPÍTULO I}

\section{INTRODUÇÃO}

\subsection{OBJETIVOS}

Muitos problemas na engenharia são caracterizados matematicamente por equações diferenciais. Problemas da mecânica dos sólidos e comportamento térmico são alguns exemplos. A dificuldade com estas equações diferenciais é que elas não podem ser resolvidas analiticamente, exceto para um conjunto restrito de casos. Assim, grande parte destes problemas é resolvida pela aplicação dos métodos numéricos de análise. Tais métodos utilizam modelos discretos em substituição aos modelos contínuos dos métodos analíticos.

Com a constante evolução dos computadores pessoais, a aplicação dos métodos numéricos na engenharia estrutural vem crescendo vertiginosamente. Há alguns anos atrás, quando não se dispunha desta importante ferramenta, a análise estrutural era feita de forma bastante simplificada. A estrutura tridimensional era dividida de tal forma que o estudo recaía na análise individual dos elementos constituintes (lajes, vigas e pilares).

Hoje, com os equipamentos computacionais disponíveis, pode-se lançar mão de modelos melhor elaborados que permitem a consideração da interação entre os diversos elementos estruturais. Isto, sem dúvida, vem fornecer cálculos mais precisos, que melhor traduzem o comportamento da estrutura.

Dada tal realidade, tem-se os seguintes objetivos no presente trabalho:

1. Apresentar uma formulação do Método dos Elementos de Contorno que permita a análise de placas combinadas a vigas ou pilares, placas estas, cujo comportamento é governado pela Teoria de Reissner; 
2. Estender a formulação de maneira a permitir a análise de estruturas em que os materiais apresentam comportamento não linear, como é o caso das estruturas executadas em concreto armado. A formulação é direcionada à análise de lajes cogumelo, onde o efeito da concentração de tensões não pode ser negligenciado.

3. Incorporar ao modelo de dano utilizado o efeito das tensões cisalhantes possibilitando uma estimativa da resistência última da estrutura. Incorporar, ainda, um modelo para absorção dos esforços advindos destas tensões, estabelecendo-se as parcelas dos esforços absorvidas pelo concreto e pela armadura transversal.

4. Desenvolver um programa computacional baseado na formulação apresentada, utilizando-se modelos constitutivos baseados nas Teorias da Plasticidade e de Dano para o aço e o concreto, respectivamente.

$\mathrm{O}$ autor deseja observar que a pesquisa ora desenvolvida teve como ponto de partida os trabalhos realizados por RIBEIRO (1992) e SILVA (1996). Ambos trabalhos de doutoramento, defendidos na Escola de Engenharia de São Carlos, da Universidade de São Paulo, sob a orientação do prof. Dr. Wilson Sérgio Venturini.

\subsection{APRESENTAÇÃO}

Dados os objetivos, passa-se à apresentação do conteúdo dos capítulos que compõem este trabalho.

$\mathrm{Na}$ finalização do presente capítulo, faz-se uma revisão bibliográfica das teorias de placas, seguida da bibliografia relativa aos primórdios dos métodos numéricos. Quanto ao Método dos Elementos de Contorno, a revisão é dividida na aplicação às teorias de Kirchhoff e de Reissner. A seguir, é apresentada a revisão bibliográfica dos modelos constitutivos aplicados ao concreto, baseados na Teoria da Plasticidade e na Mecânica do Dano.

No desenvolvimento do capítulo II apresenta-se uma introdução ao Método dos Elementos de Contorno seguida pela apresentação da teoria de placas de Reissner. O mesmo capítulo trata ainda, das equações integrais necessárias à formulação do 
problema de placas, quais sejam: Equações integrais para deslocamentos em pontos do domínio; equações integrais para deslocamentos em pontos do contorno e equações integrais para esforços nos pontos do domínio. Os termos de domínio, relativos ao carregamento, são transformados em integrais de contorno. Finalizando o capítulo, a temática é dirigida para a discretização destas equações integrais.

Objetivando a análise de pavimentos, compõe-se o capítulo III dos tópicos relativos à interação placa-apoios pontuais, placa-apoios discretos, bem como da interação placa-vigas. Neste último caso, o enrijecimento provocado pela presença das vigas, é obtido por uma combinação do Método dos Elementos de Contorno com o Método dos Elementos Finitos.

O capítulo IV explana os temas relativos à análise não linear. Apresenta-se um resumo das teorias da Plasticidade e de Dano, bem como o modelo de dano implementado no programa computacional desenvolvido.

$\mathrm{O}$ processo de solução do problema não linear é tratado no capítulo $\mathrm{V}$; o mesmo capítulo ocupa-se, ainda, da apresentação de exemplos analisados e comparados com os resultados obtidos através de outros métodos de análise e também com resultados experimentais.

No capítulo VI tem-se os tópicos relativos às conclusões, os quais compreendem uma análise da formulação desenvolvida, uma análise do desempenho dos modelos não-lineares implementados e ainda as proposições para a continuidade do trabalho desenvolvido.

No apêndice B são dados alguns fundamentos matemáticos necessários ao melhor entendimento da formulação apresentada.

\subsection{REVISÃO BIBLIOGRÁFICA}

\subsubsection{Teorias de Placas}

KIRCHHOFF (1850) desenvolveu a teoria, hoje conhecida como Teoria Clássica, aplicável a placas delgadas com pequenos deslocamentos. Com as hipóteses adotadas, a formulação leva a uma equação diferencial de quarta ordem. Nesta teoria não se consideram as deformações por cisalhamento transversal.

Posteriormente, REISSNER (1944) apresentou a formulação para placas em que considera o efeito das deformações por cisalhamento transversal, levando a um 
sistema de equações diferenciais de sexta ordem. Com este sistema satisfazem-se três condições de contorno por borda, ao invés de duas, como ocorre na Teoria Clássica.

MINDLIN (1951) apresentou uma teoria semelhante à de Reissner. Sua dedução baseou-se nas equações de equilíbrio da teoria tridimensional da elasticidade para um corpo em movimento, considerando-se a inércia rotacional e o cisalhamento transversal. O sistema de equações diferenciais obtido é, também, de sexta ordem.

SALERNO \& GOLDBERG (1960) reduziram o sistema de três equações diferenciais de Reissner a uma equação diferencial de quarta ordem semelhante à da Teoria Clássica e a uma equação diferencial de segunda ordem para determinação de uma função de tensão.

REISSNER (1976) desenvolveu estudos relativos ao problema da integração do sistema de equações para o caso de ortotropia e à influência de uma faixa limite de condições de contorno reduzidas, em função da flecha e de uma função de tensão, na solução do interior de placas isotrópicas. Com isso conseguiu distinguir efeitos de deformação por cisalhamento associados à faixa de contorno e ao domínio da placa.

LEVINSON (1980) apresentou uma nova teoria permitindo a análise estática e dinâmica de placas de espessura constante, com a consideração das deformações por cisalhamento transversal. Demonstrou também que, no caso dinâmico, sua teoria e a de Mindlin levam a uma mesma equação de onda para deslocamento transversal.

Uma nova formulação foi apresentada por REISSNER (1986) a qual pode ser imaginada como uma generalização das equações para a análise de placas com grandes deformações incluindo a deformação por cisalhamento transversal. O sistema de equações diferenciais obtido é de décima ordem, sendo duas equações simultâneas de quarta ordem, suplementadas por uma equação de segunda ordem.

REISSNER (1986) demonstrou uma teoria na qual aborda o problema de placas moderadamente espessas em que o sistema de equações diferenciais é de décima segunda ordem.

Alguns trabalhos surgiram no sentido de estimar o erro a partir da Teoria Clássica em relação à teoria exata de um problema correspondente na teoria da elasticidade. Nesta linha citam-se os trabalhos de NORDGREN (1971), (1972) e RYCHTER (1987), (1988). 
LADEVĖZE \& PECASTAINGS (1988) propuseram uma versão melhorada da teoria de Reissner para placas homogêneas, isotrópicas, com condições de contorno quaisquer. A diferença em relação à teoria de Reissner está no valor considerado do fator de deformabilidade por cisalhamento transversal e pelas condições de contorno.

BARRET \& ELLIS (1988) apresentaram o problema de placa submetida a um carregamento transversal como uma perturbação singular. No mesmo trabalho mostraram como sua teoria se relaciona com as teorias de Kirchhoff, Mindlin e Reissner.

REISSNER (1991) apresentou uma análise sistemática do problema da influência de faixas de contorno para placas ortópropas; apresentou o conceito de apoio "soft", como uma condição para a transição suave da teoria de sexta ordem para a teoria de quarta ordem.

\subsubsection{Métodos Numéricos de Análise}

O Método das Diferenças Finitas surgiu com o trabalho de SOUTHWELL (1946) sendo ainda utilizado em diversos problemas da engenharia.

O Método dos Elementos Finitos, sendo o mais difundido e utilizado, surgiu nos trabalhos desenvolvidos por TURNER (1956) E ARGYRIS \& KELSET (1960).

O Método dos Elementos de Contorno teve seu início com BETTI (1872) nos estudos de problemas utilizando equações integrais, aplicadas à teoria da elasticidade. Nesta mesma área trabalhos complementares foram desenvolvidos por SOMIGLIANA, por volta de 1880.

MUSKELISHVILI (1953), MIKHLIN (1957) e KUPRADZE (1965) continuaram utilizando equações integrais na elasticidade bidimensional, todavia, não utilizando-se ainda, as variáveis reais do problema (denominando-se, por isso, formulações indiretas). Foi RIZZO (1967) que apresentou, ainda para a elasticidade bidimensional a formulação na sua forma direta. 


\subsubsection{O Método dos Elementos de Contorno Aplicado ao Estudo de Placas}

\section{a) Aplicação à Teoria de Kirchhoff}

JASWON et al. (1967) deram início à analise de placas utilizando o Método dos Elementos de Contorno na sua forma indireta. Sua proposta foi a decomposição da equação bi-harmônica em duas equações harmônicas que, resolvidas por equações integrais e devidamente combinadas, permitem a resolução do problema.

HANSEN (1976) apresentou o método direto para a análise de placas infinitas com furos de contorno não carregado, utilizando duas equações integrais, uma correspondente à expressão do deslocamento transversal e outra à sua derivada, em relação a uma direção qualquer.

BEZINE \& GAMBY (1978) propuseram, também, uma formulação direta. Partindo da identidade de Green e considerando como variáveis os deslocamentos transversais e sua derivada na direção normal ao contorno, ou os valores correspondentes à força cortante equivalente e ao momento fletor normal ao contorno, deduziram duas equações integrais relativas ao deslocamento transversal e à sua derivada na direção normal.

BEZINE (1978) e STERN (1979) desenvolveram outros trabalhos, utilizando a formulação direta .

ALTIERO \& SIKARSKIE (1978) e WU \& ALTIERO (1979) utilizaram a formulação indireta, resolvendo problemas práticos apenas para contornos engastados. Utiliza-se, como contorno auxiliar, o contorno circular de uma placa engastada, cuja solução é conhecida.

TOTTENHAN (1979) apresentou uma discussão sobre as formulações direta e indireta para placas delgadas como também sua extensão ao caso de placas apoiadas sobre base elástica e cascas abatidas.

GUO-SHU (1986) e HARTMANN \& ZOTEMANTEL (1986) apresentaram uma formulação onde foi adotado um esquema de interpolação hermetiana para a flecha e discutidos o tratamento das integrais de domínio, a consideração de vínculos no domínio e as singularidades que ocorrem na formulação direta.

MOSHAIOV \& VORUS (1986) realizaram estudo do comportamento elastoplástico usando um esquema de carregamento incremental e com a 
consideração de momentos fletores plásticos iniciais calculados por um processo iterativo. Para efeito de avaliação das integrais de domínio, neste trabalho a placa foi dividida em células internas, admitindo-se constantes as componentes do momento plástico sobre cada uma delas.

PAIVA (1987) estudou soluções numéricas para diversos casos de carregamento, condições de contorno e posição do nó singular. Apresentou também a extensão do Método dos Elementos de Contorno para a análise de estruturas formadas por placas, vigas e pilares.

HARTLEY et al. (1988), (1989) estudaram as dificuldades relativas às singularidades que aparecem nos integrandos e a determinação de valores nos pontos internos, sugerindo um esquema de integração analítica para evitar os problemas de instabilidade numérica que podem surgir.

KATSIKADELIS \& ARMENAKAS (1989) adotaram a combinação do Método dos Elementos de Contorno com o Método das Diferenças Finitas para a solução simultânea de duas equações integrais e duas equações diferenciais.

CAMP \& GIPSON (1990) Utilizaram diversos tipos de elementos de contorno isoparamétricos, sendo as integrais calculadas analiticamente.

KARAMI et al. (1992) desenvolveram uma formulação utilizando um sistema onde são acopladas a equação bi-harmônica e uma equação harmônica, resolvidas simultaneamente sendo, ainda, as integrais de contorno calculadas analiticamente.

VABLE \& ZHANG (1992) adotaram a formulação indireta para a análise de placas, fazendo uso de um esquema de integração analítica e de funções fictícias, aproximadas por polinômios de Lagrange e Hermite.

CHUEIRI (1994) abordou a análise elastoplástica de placas, usando o método incremental-iterativo, baseado na técnica dos momentos iniciais. As integrais de domínio resultantes destes momentos foram discretizadas através da divisão do domínio em células triangulares, com função aproximadora linear. Foram propostos neste trabalho, modelos para a análise elastoplástica de lajes de concreto armado.

OLIVEIRA NETO (1998) apresentou uma nova formulação admitindo três parâmetros nodais de deslocamentos para sua representação integral: deslocamento transversal e suas derivadas nas direções normal e tangencial ao contorno. Foram usados dois valores nodais para os esforços: momento normal e força cortante 
equivalente. Obtiveram-se, dessa forma, três equações integrais de contorno por nó, obtidas a partir da discretização do contorno da placa.

\section{b) Aplicação à Teoria de Reissner}

A análise elástica de placas utilizando-se a teoria de Reissner teve seu início com WEEËN (1982). Para cada ponto do contorno foi estabelecido um sistema de três equações integrais em termos dos deslocamentos generalizados (rotação normal ao contorno, rotação tangencial e flecha) e das forças correspondentes (momento normal, momento tangencial e esforço cortante), cuja solução é obtida numericamente, sendo o contorno aproximado por elementos isoparamétricos quadráticos.

KARAM (1986), tomando como referência os trabalhos de WEEËN (1982), demonstrou a eficiência do método através de vários exemplos de placas isotrópicas, em regime elástico linear. Para a discretização do contorno foram utilizados os elementos quadrático isoparamétrico contínuo e descontínuo. Quanto a descontinuidade da normal foram utilizadas as idéias de nó duplo e de elemento descontínuo.

BARCELOS \& SILVA (1987) e WESTPHAL \& BARCELOS (1989) identificaram funções livres e funções essenciais como componentes da solução fundamental.

RIBEIRO \& VENTURINI (1989), também seguindo a formulação de WEEËN (1982), escreveram o sistema de equações lineares tomando os pontos fonte fora do domínio, evitando-se, assim, a ocorrência de algumas singularidades.

XIAO-YAN et al. (1990) consideraram a não-linearidade geométrica devido a ocorrência de grandes deslocamentos.

Em seus trabalhos, KARAN(1992) e RIBEIRO (1992) apresentaram formulação para análise não linear física, semelhante àquela proposta por MOSHAIOV \& VORUS (1986) para a Teoria Clássica.

DEBBIH et al. (1995) introduziram uma solução fundamental modificada, apropriada para placas com geometria qualquer, obtida usando-se as transformações integrais de Hankel e funções de deformação. Posteriormente, DEBBIH et al. (1995) modificaram a solução fundamental de forma que as partes das funções núcleo, 
representativas do efeito das tensões transversais, foram separadas permitindo a análise de placas espessas e finas.

SILVA (1996) apresentou uma formulação para a análise de placas com enrijecedores a qual permite a interação com outros elementos estruturais. $\mathrm{Na}$ interação placa-viga, utilizou-se uma combinação do MEC com o MEF.

ALIABADI et al. (1997) trataram da avaliação das tensões no contorno e no domínio da placa. Foram discutidos dois métodos para a avaliação das tensões no contorno. O primeiro foi baseado nas tensões e deformações locais. O segundo foi baseado na avaliação direta do tensor das tensões usando a equação integral de tensão. Avaliaram, também, as tensões internas sobre a espessura da placa comparando os resultados com as soluções dos Elementos de Contorno para o caso tridimensional.

EL-ZAFRANY (1998) discutiu o tratamento numérico das equações integrais de contorno com ênfase nos problemas de canto utilizando elementos isoparamétricos e integrais singulares com elementos de contorno constante. Demonstrou, ainda, a redução das integrais de domínio em integrais de contorno para carregamento uniforme e linearmente distribuídos, forças concentradas e momentos fletores.

PALERMO (2000) escrevendo as equações diferenciais da teoria de Mindlin com termos equivalentes aos utilizados na análise de estados planos através de dilatações e rotações, demonstrou uma conexão nas formulações do Método dos Elementos de Contorno entre esta teoria e a teoria clássica. Com isso apresentou uma formulação simples para a análise de placas, a qual permitiu a obtenção de uma solução fundamental para a teoria de Reissner/Mindlin igual à obtida por WEEËN (1982). Obteve ainda, a solução fundamental da teoria clássica quando considera-se a porção irrotacional da solução sem a correção do efeito do esforço cortante.

\subsubsection{Modelos constitutivos}

Nas últimas décadas intensivas pesquisas têm sido feitas no sentido de se formular modelos constitutivos que bem representem o comportamento do concreto armado. Modelos baseados na Teoria da Plasticidade e na Mecânica do Dano tem sido propostos nos últimos anos e se constituem em grandes avanços para a análise estrutural. 
A consideração do efeito da fissuração no concreto teve seu início no final da década de 60 e início de 70, como demonstram os trabalhos de RASHID (1968) e POPOVICS (1970).

CERVENKA (1970) propôs a aplicação da teoria da plasticidade no concreto sob compressão, usando critérios análogos aos utilizados para materiais metálicos.

JOFRIET \& McNEICE (1971) apresentaram uma formulação para análise de lajes, via Elementos Finitos, em que consideraram o efeito da fissuração. Neste trabalho assumiram uma relação momento - curvatura bilinear para representar os vários estágios de comportamento do concreto.

SCHNOBRICH et al. (1973) num estudo sobre cascas e placas de concreto armado assumiram relações tensão - deformação para o aço e o concreto. Para o aço foi considerado o comportamento elastoplástico perfeito e para o concreto elastoplástico com endurecimento na compressão, limitando-se sua tensão na tração. A placa foi considerada como uma superposição de camadas de concreto e aço e foram impostas as condições de compatibilidade entre as deformações dos materiais.

Estudo semelhante foi realizado posteriormente por SUIDAN \& SCHNOBRICH (1973) para a análise de vigas, adotando-se comportamento elastoplástico perfeito para o aço e para o concreto na compressão, limitada sua resistência à tração.

RAO \& SUBRAHMANYAN (1973) sugeriram um método para obtenção de uma relação momento - curvatura trilinear, onde a resistência do concreto tracionado entre as fissuras é considerada.

CHEN \& CHEN (1975) propuseram uma relação tensão - deformação para o concreto sob estado tridimensional de tensão. O concreto foi considerado um material elastoplástico com endurecimento e ruptura. Foram obtidas a superfície inicial de escoamento, superfícies de carregamento e superfície de ruptura e obtidas as relações incrementais tensão - deformação elastoplásticas. Tal formulação permitiu a análise mais refinada de elementos e estruturas de concreto. Modelo semelhante foi apresentado por DAMJANIC et al. (1983) os quais utilizaram a regra de fluxo associativa, fazendo aplicações ao concreto armado e protendido.

Os modelos de CHEN \& CHEN (1975) e DAMJANIC et al. (1983) diferem entre si quanto à superfície de ruptura, superfície de carregamento e regra de 
endurecimento. Na mesma linha de pesquisa, cita-se ainda o modelo proposto por CHEN \& HAN (1985) os quais adotaram uma regra de fluxo não associativa.

FIGUEIRAS (1983) desenvolveu uma formulação adotando um modelo estratificado, na qual considera a resistência do concreto tracionado entre fissuras e a capacidade de transferência de esforço cortante do concreto fissurado por encaixe dos agregados.

PROENÇA (1988) fez uma abordagem crítica dos principais modelos constitutivos aplicados ao concreto. Desenvolveu um modelo de fraturamento para o concreto, utilizando uma formulação variacional.

HU \& SCHNOBRICH (1989) propuseram um modelo onde abordaram aspectos relativos a função de escoamento, regras de endurecimento, regras de fluxo, curva tensão-deformação equivalente e módulo de endurecimento plástico. Um modelo mais refinado no qual se considera o efeito do amolecimento do concreto comprimido, a degradação das tensões no concreto paralelo à direção da fissura, dentre outros, foi proposto pelos mesmos autores em 1990.

KACHANOV (1958) foi quem introduziu o conceito de Dano a fim de modelar o efeito da fissuração distribuída na ruptura frágil em metais após um período de deformação lenta.

Comenta-se a seguir, de forma resumida, o desenvolvimento dos modelos de dano direcionados ao concreto.

Os modelos relativos ao concreto podem ser classificados como escalares (ou isótropos) e anisótropos, em função da natureza da variável de dano utilizada.

KRAJCINOVIC \& FONSEKA (1981) propuseram um modelo no qual a variável de dano é um campo vetorial expresso como uma função de coordenadas do ponto e das variáveis do estado. Esta formulação não considera as deformações permanentes devidas ao processo de dano.

LADEVÈZE (1983) propôs um modelo racional que permite a determinação da natureza matemática da variável de dano interna. Neste caso a variável de dano é um tensor de quarta ordem.

MAZARS (1984) apresentou o modelo escalar, indicado para estudo de processo de dano do concreto submetido a um carregamento proporcional ou cíclico. Identifica-se a variável de dano em função de uma deformação equivalente, que 
caracteriza o estado local de extensões do material. Esta variável é definida de tal forma a recuperar, como um caso particular, os resultados experimentais dos testes de tração e compressão uniaxiais: os mesmos servem de base para identificar os cinco parâmetros do modelo.

O modelo anisótropo de ORTIZ (1985) permite a representação de algumas características do comportamento do concreto associado ao dano. Neste modelo, o concreto é tratado como um material composto formado por uma fase argamassa e uma fase agregado, cada uma das quais com uma lei constitutiva particular. Assumese para o agregado um modelo elastoplástico não-associativo; para a argamassa prevalece o modelo de dano.

PAPA (1990) estendeu as proposições de Mazars objetivando reproduzir a resposta do material em presença de carregamento de fadiga, incluindo a presença de deformações permanentes.

ÁLVARES (1993) estudou o modelo proposto por Mazars para análise do comportamento de elementos estruturais lineares em concreto. Realizou a identificação dos parâmetros os quais se mostraram próximos àqueles originalmente sugeridos. Foi realizada a implementação numérica via elementos finitos, cujos resultados foram comparados com os resultados experimentais realizados. Tal análise demonstrou o bom desempenho do modelo em estudo.

FLÓREZ-LÓPEZ (1993) apresentou um modelo para a simulação do colapso em pórticos planos considerando-se os efeitos de dano e plasticidade localizados. Neste modelo os efeitos da não linearidade concentram-se apenas nas extremidades dos elementos. Nesta mesma linha, ÁLVARES (1998) propôs um novo modelo, onde considerou que os processos de danificação, associados à flexão e restritos às extremidades dos elementos, são interdependentes e afetam os coeficientes de transmissão de esforços ao longo do elemento. 


\section{CAPÍTULO II}

\section{O MÉTODO DOS ELEMENTOS DE CONTORNO APLICADO ÀS PLACAS DE REISSNER}

\subsection{INTRODUÇÃO AO MÉTODO DOS ELEMENTOS DE CONTORNO}

Há muitos problemas na Engenharia que podem ser caracterizados matematicamente por equações diferenciais, com as devidas condições de contorno. Problemas da mecânica dos sólidos e fluidos e comportamento térmico são alguns destes problemas. A dificuldade com estas equações diferenciais é que elas não podem ser resolvidas analiticamente, exceto para um conjunto restrito de casos.

Para a obtenção da solução destes problemas faz-se necessário o uso dos métodos numéricos. Tais métodos podem ser classificados, segundo BECKER (1992), em: Método das Diferenças Finitas (MDF), Método dos Elementos Finitos (MEF) e Método dos Elementos de Contorno (MEC), como representado na figura 2.1, cada um dos quais com suas particularidades, vantagens e desvantagens. 


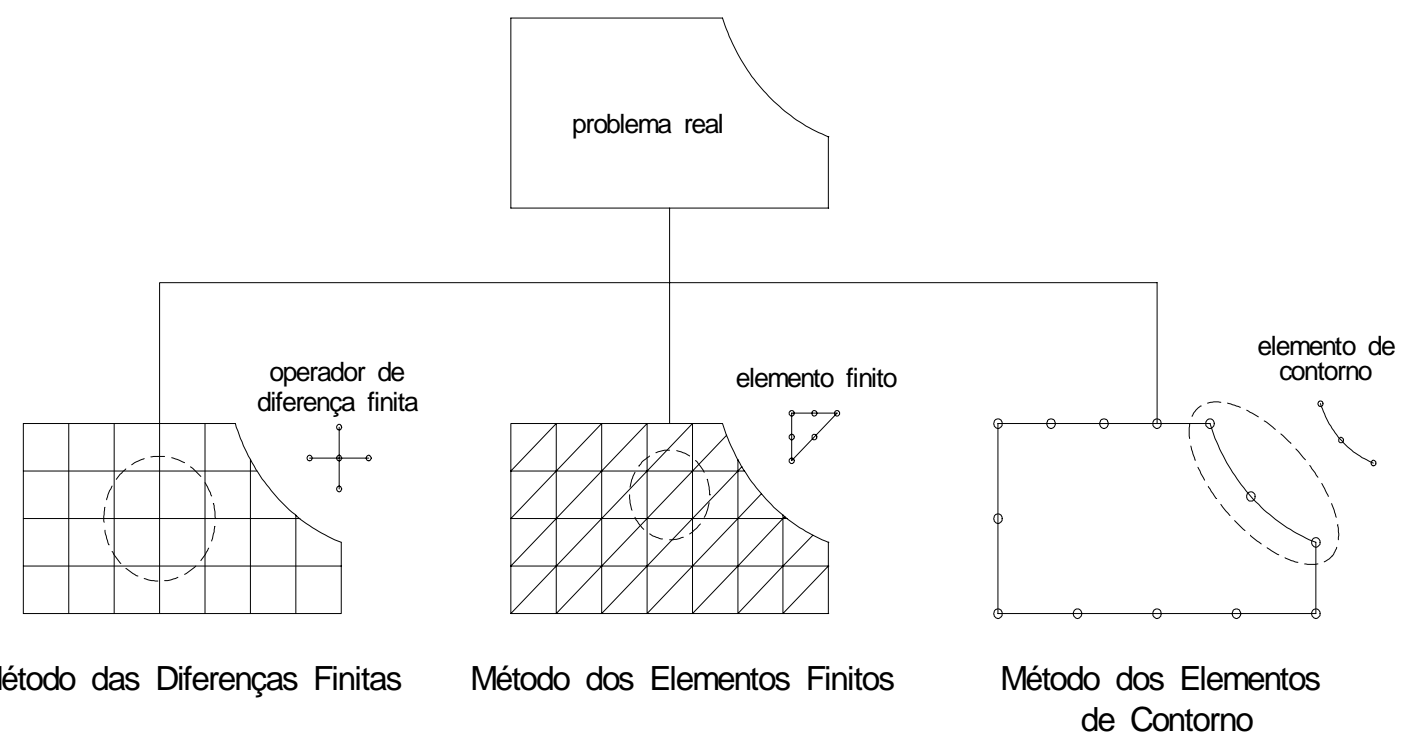

FIGURA 2.1. Métodos numéricos aplicados aos problemas de Engenharia. (KANE, 1994)

\section{a) Método das Diferença Finita}

O conceito básico deste método consiste da transformação das equações diferenciais que governam o problema em um sistema de equações algébricas, pela aplicação do operador de diferença. Este operador transforma todas as derivadas contínuas em variáveis sobre uma célula. Os pontos nodais onde as incógnitas são associadas devem ser prescritos e especificadas as funções interpoladoras que governam as derivadas. O sistema final de equações é constituído por uma matriz banda pequena e um vetor independente, que é computado de acordo com as condições de contorno prescritas.

O Método das Diferenças Finitas é o mais simples dos três métodos e relativamente fácil de programar. Sua principal desvantagem em problemas práticos é que ela não é apropriada para geometrias irregulares, e em problemas cujas incógnitas variam rapidamente, como problemas de concentração de tensões. 


\section{b) Método dos Elementos Finitos}

Este método consiste da subdivisão do domínio (discretização física) em elementos (daí o nome de 'Elementos Finitos'), cada um dos quais tratados como um contínuo menor. Associam-se nós a cada elemento e escolhem-se funções interpoladoras que aproximam a resposta real do domínio. Uma vez que estas funções são definidas em função dos valores nodais, as equações do elemento finito são facilmente obtidas através do princípio variacional ou pela técnica dos resíduos ponderados. O uso de qualquer um destes procedimentos produz um sistema simétrico de equações.

O Método dos Elementos Finitos é muito apropriado para problemas de formas geométricas complexas. Para obterem-se bons resultados em regiões onde as variáveis mudam rapidamente, deve-se usar um maior número de elementos.

\section{c) Método dos Elementos de Contorno}

No Método dos Elementos de Contorno, as equações diferenciais governantes são transformadas em equações integrais equivalentes. Através do Teorema Divergente, ou de Gauss-Green, estas equações integrais, envolvendo equações integrais de volume e de superfície, são transformadas em equações integrais de superfície apenas (equações integrais de contorno). Esta última transformação envolve certas soluções conhecidas (soluções fundamentais) para a equação diferencial original. As equações integrais são então substituídas por um conjunto similar de equações integrais discretizadas, onde são usadas as incógnitas do contorno em um conjunto finito de nós. Entre estes nós estão os elementos de contorno, onde a solução dentro de cada elemento é dada em função dos valores nodais e por uma função de interpolação simples.

O Método dos Elementos de Contorno pode ser facilmente ajustável a contornos geometricamente complexos. Além do mais, uma vez que todas as aproximações estão restritas ao contorno, pode-se modelar regiões com gradientes elevados com melhor exatidão em relação ao Método dos Elementos Finitos.

$\mathrm{Na}$ obtenção do Método dos Elementos de Contorno, tomando-se como exemplo um problema elástico, segue-se, em linhas gerais, a seguinte itemização (BECKER, 1992): 
1. Obtêm-se as equações diferenciais de deslocamentos sobre o domínio (equações de Navier). Para tanto, substituem-se as relações deformação-deslocamento nas equações constitutivas (lei de Hooke) e estas nas equações diferenciais de equilíbrio.

2. Obtém-se a solução fundamental para as equações diferenciais. Esta é uma solução que deve ser aplicável a qualquer geometria e é baseada na solução de uma carga pontual em um meio infinito. A solução fundamental é da ordem de (1/r) ou $\ln (1 / \mathbf{r})$, onde $\mathbf{r}$ é a distância física entre o ponto de aplicação da carga (definido como "ponto de carga", $\xi$ ) e qualquer outro ponto sobre o contorno (definido como "ponto campo", $\mathbf{x}$ ).

3. Utiliza-se o Teorema da Reciprocidade de Betti. Este teorema estabelece que se dois estados de tensão (a) e (b) existem que satisfaçam o equilíbrio, então o trabalho realizado pelas forças do sistema (a) sobre os deslocamentos do sistema (b) é igual ao trabalho realizado pelas forças de (b) sobre os deslocamentos de (a):

$$
\sum_{\mathbf{p}} \mathbf{F}_{\mathbf{p}}^{(\mathbf{a})} \mathbf{u}_{\mathbf{p}}^{(\mathbf{b})}=\sum_{\mathbf{p}} \mathbf{F}_{\mathbf{p}}^{(\mathbf{b})} \mathbf{u}_{\mathbf{p}}^{(\mathbf{a})}
$$

onde p é qualquer ponto submetido a uma força $\mathbf{F}$. Se as forças externas estiverem apenas sobre a superfície $\Gamma$, então é possível obter-se a seguinte equação integral:

$$
\int_{\Gamma} \mathbf{t}_{\mathbf{i}}^{(\mathbf{a})} \mathbf{u}_{\mathbf{i}}^{(\mathbf{b})} \mathbf{d} \Gamma=\int_{\Gamma} \mathbf{t}_{\mathbf{i}}^{(\mathbf{b})} \mathbf{u}_{\mathbf{i}}^{(a)} d \Gamma
$$

onde $\mathbf{i}=\mathbf{1}, \mathbf{2}, \mathbf{3}$ (correspondentes às coordenadas cartesianas $\mathbf{x}, \mathbf{y}, \mathbf{z}$ ). Escolhemse o sistema (a) como solução fundamental (conhecida) e o sistema (b) como o problema real (incógnito), o resultado é uma equação integral de contorno relacionando os deslocamentos e tensões na superfície.

4. Divide-se a superfície em segmentos ou elementos. Usam-se funções interpoladoras para descrever a geometria e variáveis sobre cada elemento. Tais funções podem ser lineares, quadráticas ou de ordem superior. Dada a complexidade das funções integrais, a integração analítica é substituída pela integração numérica, utilizando-se a técnica da quadratura de Gauss. Esquemas especiais são necessários para integrar os termos singulares quando os pontos 
nodais estão muito próximos uns dos outros ou quando o ponto de carga, $\boldsymbol{\xi}$, coincide com o ponto do contorno, $\mathbf{x}$. Isto ocorre em função de que a solução fundamental contém termos da ordem 1/r. Somando-se as integrais sobre cada elemento, tem-se a avaliação de toda a superfície.

5. Forma-se a solução matricial repetindo-se o processo de integração com o ponto de carga, $\xi$, localizado em cada ponto nodal da superfície. O ponto de carga $\xi$, (solução fundamental) é colocado primeiro no nó 1, produzindo apenas um conjunto de equações relacionando todas as $\mathbf{N}$ variáveis sobre a superfície. Um conjunto de equações em problemas tri-dimensionais contém três equações. A seguir o ponto de carga é colocado no nó $\mathbf{2}$, produzindo outro conjunto de equações, e assim sucessivamente, até que todos os $\mathbf{N}$ conjuntos de equações estejam formados. O sistema resultante de equações lineares tem a seguinte forma:

$$
[\mathrm{A}][\mathbf{u}]=[\mathbf{B}][\mathbf{t}]
$$

6. Aplicam-se as condições de contorno. Estas assumem a forma de deslocamentos prescritos ou forças prescritas. Re-arranjam-se as equações lineares de tal forma que as variáveis incógnitas estejam do lado esquerdo e todos os valores conhecidos, do lado direito. Obtém-se a seguinte equação matricial modificada:

$$
\left[\mathrm{A}^{*}\right][\mathrm{x}]=\left[\mathrm{B}^{*}\right][\mathrm{y}]=[\mathrm{c}]
$$

onde o vetor $[\mathrm{x}]$ incógnito contém uma mistura de deslocamentos e tensões, enquanto que [y] contém todos os valores prescritos de deslocamentos e tensões.

7. Resolve-se o sistema geral de equações lineares. Como a matriz dos coeficientes é assimétrica e povoada com termos não nulos, pode-se usar técnicas diretas de solução (tal como eliminação de Gauss).

8. Obtêm-se informações adicionais. Determinados os deslocamentos e tensões no contorno, pode-se calcular deformações, deslocamentos e forças nos pontos internos. 


\subsubsection{Equações de Navier}

Seja um elemento infinitesimal de um corpo em equilíbrio. Para este elemento as equações de equilíbrio podem ser expressas por:

$$
\sigma_{i j, j}+f_{i}=0
$$

As relações deformação - deslocamento são dadas por:

$$
\varepsilon_{\mathrm{ij}}=\frac{1}{2}\left(u_{\mathrm{i}, \mathrm{j}}+\mathrm{u}_{\mathrm{j}, \mathrm{i}}\right)
$$

A lei de Hooke, relacionando tensões a deformações, pode ser escrita:

$$
\sigma_{i j}=\frac{2 G v}{1-2 v} \delta_{i j} \varepsilon_{m m}+2 G \varepsilon_{i j}
$$

Fazendo-se a substituição da equação (2.6) em (2.7) e o resultado em (2.5), obtém-se a equação diferencial em termos de deslocamentos:

$$
\mathbf{G u}_{\mathrm{i}, \mathrm{jj}}+\left(\frac{G}{1-2 v}\right) \mathbf{u}_{j, \mathrm{ij}}=-\mathbf{f}_{\mathrm{i}}
$$

A equação (2.8) é conhecida como equação de Navier, que na sua forma vetorial é dada por:

$$
\nabla^{2} \mathbf{u}+\frac{1}{1-2 v} \nabla(\nabla \cdot \mathbf{u})=-\frac{\mathbf{f}}{\mathbf{G}}
$$

\subsubsection{Solução fundamental}

a) Vetor de Galerkin

A vantagem das equações (2.8) é que as 15 relações mostradas foram manipuladas, resultando em apenas 3 equações diferenciais que têm como incógnitas apenas deslocamentos $\mathbf{u}_{\mathbf{i}}$. O problema com estas equações é que cada uma delas está em função das 3 componentes de deslocamentos $\mathbf{u}_{1}, \mathbf{u}_{2}$ e $\mathbf{u}_{3}$, tornando difícil a solução analítica. Uma forma de resolver este problema é expressar o vetor deslocamento em termos de outro vetor, o vetor de Galerkin, $\mathbf{G}_{\mathbf{i}}$ :

$$
2 G_{i}=2(1-v) G_{i, j j}-G_{i, j i}
$$


Substituindo-se a eq.(2.10) na eq. (2.8), obtém-se as equações diferenciais de equilíbrio em termos do vetor de Galerkin:

$$
(1-v) G_{i, k k j j}+f_{i}=0
$$

Observe-se que cada equação representada por (2.11) está relacionada com apenas uma componente do vetor de Galerkin, podendo-se obter soluções analíticas.

Encontradas as componentes do vetor de Galerkin que satisfaçam a eq. (2.11), determinam-se as componentes do vetor dos deslocamentos $\mathbf{u}_{\mathbf{i}}$, utilizando-se a eq. (2.10).

A forma vetorial da eq. (2.11) é:

$$
\nabla^{4} G=\nabla^{2}\left(\nabla^{2} G\right)=-\frac{1}{(1-v)} f
$$

conhecida como equação bi-harmônica quando a força de massa é nula.

A expressão das componentes de tensão em função do vetor de Galerkin é obtida substituindo-se a relação deformação-deslocamento dada pela eq. (2.6) na relação tensão-deformação dada pela eq. (2.7) fornecendo:

$$
\sigma_{i j}=\frac{2 G v}{1-2 v} \delta_{i j} u_{k, k}+G\left(u_{i, j}+u_{j, i}\right)
$$

Substituindo-se a eq.(2.10) na eq.(2.13), chega-se a:

$$
\sigma_{i j}=(1-v)\left[G_{i, k k j}+G_{j, k k i}\right]-G_{k, k i j}+v \delta_{i j} G_{1, k k k}
$$

\section{b) Problema de Kelvin}

O problema de uma carga concentrada aplicada no interior de um domínio infinito é conhecido como problema de Kelvin. Suponha que uma força unitária é aplicada sobre um ponto interior $\xi$; procura-se o efeito desta força em outro ponto $\mathbf{x}$, qualquer do domínio. Simplificadamente, procura-se saber como $\mathbf{x}$ "sente" o efeito da força em $\xi$. A solução deve satisfazer duas condições:

1. as tensões devem ser nulas quando a distância entre $\xi$ e $\mathbf{x}$ tender ao infinito;

2. as tensões devem ser 'singulares' no próprio ponto $\xi$, ou seja, tendem ao infinito quando a distância entre $\xi$ e $\mathbf{x}$ tende a zero. 
Verifica-se que o vetor de Galerkin dado a seguir é uma solução da eq.(2.12) e satisfaz ambas as condições físicas mencionadas:

$$
G_{i}=\frac{1}{8 \pi(1-v)} r(\xi, x)
$$

A função $\mathbf{r}(\boldsymbol{\xi}, \mathbf{x})$ é a distância física entre os pontos $\boldsymbol{\xi}$ e $\mathbf{x}$.

$\mathrm{O}$ vetor deslocamento, $\mathbf{u}_{\mathbf{i}}$, é obtido substituindo-se a eq.(2.15) na eq.(2.10):

$$
u_{i}=\frac{1}{16 \pi G(1-v)} r(\xi, x)^{-1}\left[(3-4 v) \delta_{i k}+r(\xi, x)_{, i} r(\xi, x)_{, k}\right]
$$

Substituindo-se a eq.(2.15) na eq.(2.14) obtém-se as componentes de tensão:

$$
\begin{aligned}
& \sigma_{i j}=\frac{(1-2 v)}{8 \pi(1-v)} \mathbf{r}(\xi, x)^{-2}\left[\delta_{i j} r(\xi, x)_{, k}-\delta_{j k} r(\xi, x)_{, i}-\delta_{k i} r(\xi, x)_{, j}-\right. \\
& \left.+\frac{3}{1-2 v} \mathbf{r}(\xi, \mathbf{x})_{, \mathbf{i}} \mathbf{r}(\xi, \mathbf{x})_{, j} \mathbf{r}(\xi, x)_{, \mathbf{k}}\right]
\end{aligned}
$$

Finalmente, as componentes do vetor de tensão sobre uma superfície com normal $\mathbf{n}$ podem ser obtidas utilizando-se a transformação de tensão de Cauchy:

$$
\begin{aligned}
\mathbf{t}_{\mathrm{i}}= & \frac{-1}{8 \pi(1-v)} \mathbf{r}(\xi, x)^{-2}\left\{\mathbf { r } ( \xi , x ) _ { , j } \mathbf { n } _ { j } \left[(1-2 v) \delta_{k i}+\right.\right. \\
& \left.\left.+3 r(\xi, x)_{, i} \mathbf{r}(\xi, x)_{, k}\right]+(1-2 v)\left[r(\xi, x)_{, i} n_{k}-r(\xi, x)_{, k} n_{i}\right]\right\}
\end{aligned}
$$

\subsubsection{Teorema da Reciprocidade de Betti}

Considere um corpo de domínio $\boldsymbol{\Omega}$ e superfície $\Gamma$, submetido a dois sistemas diferentes de carregamento e com dois correspondentes conjuntos de deformações em equilíbrio: sistema (1) de tensões aplicadas $\boldsymbol{\sigma}_{\mathbf{i j}}{ }^{(\mathbf{1})}$, que dá origem a um conjunto de deformações $\boldsymbol{\varepsilon}_{\mathbf{i j}}{ }^{(\mathbf{1})}$; sistema (2) de tensões aplicadas $\boldsymbol{\sigma}_{\mathrm{ij}}{ }^{(\mathbf{2})}$ que dá origem a um conjunto de deformações $\boldsymbol{\varepsilon}_{\mathrm{ij}}{ }^{(2)}$. O teorema da reciprocidade de Betti estabelece que o trabalho realizado pelo sistema de tensões (1) sobre as deformações do sistema (2) é igual ao trabalho realizado pelo sistema de tensões (2) sobre as deformações do sistema (1), ou seja:

$$
\int_{\Omega} \sigma_{\mathrm{ij}}^{(1)} \varepsilon_{\mathrm{ij}}^{(2)} \mathrm{d} \Omega=\int_{\Omega} \sigma_{\mathrm{ij}}^{(2)} \varepsilon_{\mathrm{ij}}^{(1)} \mathrm{d} \Omega
$$

Desenvolvendo a eq.(2.19) é possível obter a seguinte expressão para o teorema de Betti: 


$$
\int_{\Gamma} p_{i}^{(1)} u_{i}^{(2)} d \Gamma+\int_{\Omega} f_{i}^{(1)} u_{i}^{(2)} d \Omega=\int_{\Gamma} p_{i}^{(2)} u_{i}^{(1)} d \Gamma+\int_{\Omega} f_{i}^{(2)} u_{i}^{(1)} d \Omega
$$

\subsubsection{Identidade Somigliana}

A eq.(2.20) pode ser transformada em uma equação integral de contorno, apenas. O sistema (1) pode ser entendido como o problema real em consideração; o sistema (2) pode ser entendido como o problema de Kelvin, para a carga unitária na posição $\xi$, de um meio infinito. Abandonando-se os superscritos, pode-se escrever:

$$
\int_{\Gamma} p_{i} u_{i k} d \Gamma+\int_{\Omega} f_{i} u_{i k} d \Omega=\int_{\Gamma} p_{i k} u_{i} d \Gamma+\int_{\Omega} \delta_{i k}(x-\xi) u_{i} d \Omega
$$

Observe-se que os valores da solução fundamental, em deslocamento u e forças de superfície p, têm um subscrito extra. As quantidades com subscritos simples referem-se ao problema real. Observe-se ainda, que a carga concentrada no problema de Kelvin foi suposta aplicada como uma força de massa na forma de uma função Delta. Utilizando-se a propriedade seletiva da função Delta de Dirac, tem-se:

$$
\int_{\Gamma} p_{i} u_{i k} d \Gamma+\int_{\Omega} f_{i} u_{i k} d \Omega=\int_{\Gamma} p_{i k} u_{i} d \Gamma+C_{i k} u_{i}(\xi)
$$

O símbolo $\mathbf{C}_{\mathbf{i k}}$ foi introduzido para computar os casos onde a localização $\boldsymbol{\xi}$ da carga concentrada esteja dentro ou fora de $\Omega$ :

$$
\mathbf{C}_{\mathrm{ik}}=\left\{\begin{array}{l}
\mathbf{0} \text { para } \xi \text { fora de } \boldsymbol{\Omega} ; \\
\boldsymbol{\delta}_{\mathrm{ik}} \text { para } \xi \text { dentro de } \Omega ; \\
\frac{\mathbf{1}}{\mathbf{2}} \boldsymbol{\delta}_{\mathrm{ik}} \text { para } \xi \text { sobre uma parte suave de } \Gamma
\end{array}\right.
$$

No caso da não consideração das forças de massa, a eq.(2.22) torna-se:

$$
\int_{\Gamma} p_{i} u_{i k} d \Gamma=\int_{\Gamma} p_{i k} u_{i} d \Gamma+C_{i k} u_{i}(\xi)
$$

A eq.(2.24) é conhecida como Identidade Somigliana e estabelece que a componente de deslocamento de um corpo num ponto $\xi$ é dada pelas integrações das tensões e deslocamentos multiplicados pelas soluções fundamentais, com o ponto de carga localizado em $\xi$. 


\subsection{TEORIA DE PLACAS DE REISSNER}

A teoria de Reissner, comentada de forma sucinta neste item, conduz a um problema de integração de sexta ordem que deve satisfaz a três condições de contorno por borda.

\subsubsection{Hipóteses básicas}

As hipóteses através das quais a Teoria de Reissner se fundamenta são:

a) a espessura da placa é pequena, quando comparada com as outras dimensões;

b) a placa é constituída de material homogêneo e isótropo, e de comportamento elástico-linear;

c) em detrimento da consideração do efeito das deformações por esforço cortante, uma reta inicialmente normal ao plano médio da placa, após a deformação permanece reta, porém, não necessariamente normal à superfície média;

d) as componentes tangenciais do tensor das tensões são nulas nas faces da placa;

e) as componentes normais às superfícies externas da placa são $\boldsymbol{\sigma}_{\mathbf{3 3}}=\mathbf{\pm q} / \mathbf{2}$, para $\mathbf{x}_{\mathbf{3}}$ $= \pm \mathbf{h} / \mathbf{2}$, sendo $\mathbf{q}$ o carregamento transversal aplicado à placa e $\mathbf{h}$ sua espessura.

\subsubsection{Sistema de Equações de Reissner}

A base da teoria de Reissner é um sistema composto por três equações de equilíbrio dadas por:

$$
\mathbf{M}_{\alpha \beta, \beta}-\mathbf{Q}_{\alpha}=\mathbf{0} ; \quad \mathbf{Q}_{\alpha, \alpha}+\mathbf{q}=\mathbf{0} \quad(\alpha, \beta=1,2)
$$

e pelas cinco equações dos esforços em função dos deslocamentos generalizados:

$$
\begin{gathered}
M_{\alpha \beta}=\mathbf{D} \frac{(1-v)}{2}\left(\phi_{\alpha, \beta}+\phi_{\beta, \alpha}+\frac{2 v}{1-v} \phi_{\gamma, \gamma} \delta_{\alpha \beta}\right)+\frac{v q}{(1-v) \lambda^{2}} \delta_{\alpha \beta} \\
Q_{\alpha}=D \frac{(1-v)}{2} \lambda^{2}\left(\phi_{\alpha}+w_{, \alpha}\right)
\end{gathered}
$$

onde $\lambda=\sqrt{\mathbf{1 0}} / \mathbf{h}$ é uma constante característica das equações de Reissner; $\mathbf{v}$ é o coeficiente de Poisson; $\mathbf{D}=\mathbf{E} \mathbf{h}^{3} /\left(\mathbf{1 2}\left(\mathbf{1}-\mathbf{v}^{\mathbf{2}}\right)\right)$ é a rigidez à flexão da placa e $\mathbf{E}$ é o módulo de elasticidade longitudinal.

Estas equações formam um sistema de oito equações diferenciais de primeira ordem, onde os oito incógnitos são os momentos $\mathbf{M}_{11}, \mathbf{M}_{12}$ e $\mathbf{M}_{22}$, os esforços cortantes $\mathbf{Q}_{1}$ e $\mathbf{Q}_{2}$, o deslocamento $\mathbf{w}$ e as rotações $\boldsymbol{\Phi}_{\mathbf{1}}$ e $\boldsymbol{\Phi}_{\mathbf{2}}$. 
É possível condensar essas oito equações, obtendo-se um outro sistema de três equações diferenciais parciais de ordem superior:

$$
\begin{gathered}
Q_{1}-\frac{1}{\lambda^{2}} \nabla^{2} Q_{1}+\frac{1}{\lambda^{2}(1-v)} \frac{\partial q}{\partial x_{1}}=-D \frac{\partial}{\partial x_{1}} \nabla^{2} w \\
Q_{2}-\frac{1}{\lambda^{2}} \nabla^{2} Q_{2}+\frac{1}{\lambda^{2}(1-v)} \frac{\partial q}{\partial x_{2}}=-D \frac{\partial}{\partial x_{2}} \nabla^{2} w \\
D \nabla^{4} w=q-\frac{(2-v)}{\lambda^{2}(1-v)} \nabla^{2} q
\end{gathered}
$$

onde $\nabla^{2}=\partial^{2} /(\partial \mathbf{x} \partial \mathbf{x})$ é o operador de Laplace.

Deve-se salientar que, admitida a ocorrência de momentos distribuídos na superfície média da placa de intensidade $\mathbf{m}_{\boldsymbol{\alpha}}$, as equações de equilíbrio passam a ser:

$$
\mathbf{M}_{\alpha \beta, \beta}-\mathbf{Q}_{\alpha}+\mathbf{m}_{\alpha}=\mathbf{0} ; \quad \mathbf{Q}_{\alpha, \alpha}+\mathbf{q}=\mathbf{0}
$$

Admitindo-se a possibilidade de ocorrência de ações devidas a campos de deformações iniciais (efeitos de temperatura, por exemplo), o tensor de deformações pode ser entendido como a soma das seguintes parcelas:

$$
\varepsilon_{\mathrm{ij}}=\varepsilon_{\mathrm{ij}}^{\mathrm{e}}+\varepsilon_{\mathrm{ij}}^{\mathrm{o}}
$$

onde $\varepsilon_{\mathrm{ij}}^{\mathbf{e}}$ é a parcela elástica devida ao carregamento direto e às condições de contorno prescritas. A parcela $\boldsymbol{\varepsilon}_{\mathbf{i j}}^{\mathbf{o}}$ representa o campo de deformações iniciais. No caso de placas, esta parcela corresponde a um campo de curvaturas iniciais, o qual é transformado em um campo de momentos iniciais da seguinte forma:

$$
\mathbf{M}_{\alpha \beta}^{\mathbf{o}}=\mathbf{C}_{\alpha \beta \gamma \theta} \chi_{\gamma \theta}^{\mathbf{o}}
$$

$\mathbf{C}_{\boldsymbol{\alpha} \beta \boldsymbol{\gamma} \boldsymbol{\theta}}$ são as componentes do tensor constitutivo de Quarta ordem e $\boldsymbol{\chi}_{\boldsymbol{\gamma} \boldsymbol{\theta}}^{\mathbf{0}}$ são as componentes do campo de curvaturas iniciais. Isto posto, tem-se a seguinte relação entre os momentos:

$$
\mathbf{M}_{\alpha \beta}=\mathbf{M}_{\alpha \beta}^{\mathrm{e}}-\mathbf{M}_{\alpha \beta}^{\mathbf{o}}
$$

sendo $\mathbf{M}_{\boldsymbol{\alpha} \beta}^{\mathrm{e}}$ o momento total calculado elasticamente e, $\mathbf{M}_{\alpha \beta}$, o momento verdadeiro, computando-se o carregamento direto e o campo de curvaturas iniciais. Logo, tendose em vista a eq.(2.26), pode-se escrever: 
$M_{\alpha \beta}=D \frac{(1-v)}{2}\left(\phi_{\alpha, \beta}+\phi_{\beta, \alpha}+\frac{2 v}{(1-v)} \phi_{\gamma, \gamma} \delta_{\alpha \beta}\right)+\frac{v q}{(1-v) \lambda^{2}} \delta_{\alpha \beta}-M_{\alpha \beta}^{0}$

Fazendo-se: $\quad \overline{\mathbf{M}}_{\alpha \beta}^{\mathrm{e}}=\mathbf{D} \frac{1-v}{2}\left(\phi_{\alpha, \beta}+\phi_{\beta, \alpha}+\frac{2 v}{1-v} \phi_{\gamma, \gamma} \delta_{\alpha \beta}\right)$

$$
\mathrm{e} \quad \overline{\mathbf{M}}_{\alpha \beta}^{\mathrm{e}}=\mathbf{C}_{\alpha \beta \gamma \theta} \chi_{\gamma \theta}
$$

com:

$$
\chi_{\gamma \theta}=\phi_{\gamma, \theta}
$$

obtém-se, para a eq.(2.32):

$$
M_{\alpha \beta}=\bar{M}_{\alpha \beta}^{\mathrm{e}}+\frac{v q}{(1-v) \lambda^{2}} \delta_{\alpha \beta}-M_{\alpha \beta}^{0}
$$

O esforço cortante pode ser expresso por:

$$
\mathbf{Q}_{\alpha}=\mathrm{C}_{3 \alpha 3 \theta} \Psi_{3 \theta}
$$

onde $\Psi_{3 \theta}$ são as deformações por esforço cortante. $\chi_{\gamma \theta}$ são as componentes de curvatura devidas ao carregamento.

\subsubsection{Condições de contorno}

O sistema de equações diferenciais envolvido na teoria da Reissner satisfaz a três condições físicas em cada ponto do contorno.

As condições de contorno usuais para placas retangulares são: borda simplesmente apoiada, borda engastada e borda livre.

No caso de borda simplesmente apoiada, pode-se prescrever duas formas distintas de condições de contorno:

$$
\begin{array}{llllll}
\mathbf{w}=\mathbf{0}, & \mathbf{M}_{\mathbf{n}}=\mathbf{0} & \text { e } & \boldsymbol{\Phi}_{\mathrm{s}}=\mathbf{0} & \text { ("hard condition") } \\
\mathbf{w}=\mathbf{0}, & \mathbf{M}_{\mathbf{n}}=\mathbf{0} & \text { e } & \mathbf{M}_{\mathrm{ns}}=\mathbf{0} & \text { ("soft condition") }
\end{array}
$$

onde $\mathbf{n}$ e $\mathbf{s}$ são os eixos normal e tangencial ao contorno, respectivamente.

A primeira condição prescreve a rotação tangencial nula na borda, que é normalmente usada na teoria clássica de Kirchhoff. A segunda já prescreve o momento de torção, $\mathbf{M}_{\mathrm{n}}$, nulo no contorno.

Para borda engastada, tem-se: $\mathbf{w}=\mathbf{0}, \quad \boldsymbol{\Phi}_{\mathbf{n}}=\mathbf{0}$ e $\quad \boldsymbol{\Phi}_{\mathbf{s}}=\mathbf{0}$.

Para borda livre, tem-se: $\mathbf{M}_{\mathbf{n}}=\mathbf{0}, \quad \mathbf{M}_{\mathrm{ns}}=\mathbf{0} \quad$ e $\quad \mathbf{Q}_{\mathbf{n}}=\mathbf{0}$. 


\subsection{EQUAÇÕES INTEGRAIS}

Apresentam-se neste item as equações integrais de placas pela Teoria de Reissner, necessárias à formulação do problema do Método dos Elementos de Contorno.

\subsubsection{Equações integrais para deslocamentos em pontos do domínio.}

Considere-se uma placa de espessura h, constante, em equilíbrio, sob as ações de um carregamento transversal distribuído, q, um campo de tensões iniciais generalizadas, caracterizado pelas componentes de momentos $\mathbf{M}_{\alpha \beta}^{\mathbf{0}}$, e momentos externos distribuídos, $\mathbf{m}_{\boldsymbol{\alpha}}$, todos aplicados no domínio. Considere-se, ainda, uma placa infinita de domínio $\Omega^{*}$ e contorno $\Gamma^{*}$, também em equilíbrio e que contém a placa finita.

Utilizando-se o Teorema de Reciprocidade de Betti ou o Método dos Resíduos Ponderados, deduzem-se as seguintes equações integrais para deslocamentos em pontos do domínio:

$$
\begin{aligned}
& \mathbf{u}_{\mathrm{i}}(\xi)=\int_{\Gamma}\left[\mathbf{u}_{\mathrm{ij}}^{*}(\xi, \mathbf{x}) \mathbf{p}_{\mathrm{j}}(\mathbf{x})-\mathbf{p}_{\mathrm{ij}}^{*}(\xi, \mathbf{x}) \mathbf{u}_{\mathrm{j}}(\mathbf{x})\right] \mathrm{d} \Gamma(\mathbf{x})+\int_{\Omega_{\mathrm{q}}}\left[\mathbf{u}_{\mathrm{i} 3}^{*}(\xi, \mathbf{x})-\right. \\
& \left.+\frac{v}{(1-v) \lambda^{2}} \mathbf{u}_{\mathrm{i} \alpha, \alpha}^{*}(\xi, \mathbf{x})\right] \mathbf{q}(\mathbf{x}) \mathrm{d} \Omega(\mathbf{x})+\int_{\Omega} \mathbf{u}_{\mathrm{i} \alpha, \beta}^{*}(\xi, \mathbf{x}) \mathbf{M}_{\alpha \beta}^{\mathbf{o}}(\mathbf{x}) \mathrm{d} \Omega(\mathbf{x})+ \\
& +\int_{\Omega_{\mathrm{m}}} \mathbf{u}_{\mathrm{i} \alpha}^{*}(\xi, \mathbf{x}) \mathbf{m}_{\alpha}(\mathbf{x}) \mathrm{d} \Omega(\mathbf{x})
\end{aligned}
$$

onde

$\xi$ : é o ponte fonte onde são aplicadas as cargas concentradas generalizadas; $\mathbf{x}$ : ponto campo, onde são observados os efeitos das cargas unitárias aplicadas; $\mathbf{u}_{\mathbf{i j}}^{*}(\boldsymbol{\xi}, \mathbf{x})$ : deslocamento generalizado na direção $\mathbf{i}$ do ponto $\mathbf{x}$, correspondente a uma força generalizada concentrada unitária aplicada na direção $\mathbf{j}$ do ponto $\xi ; \mathbf{p}_{\mathbf{i j}}^{*}(\boldsymbol{\xi}, \mathbf{x})$ : força de superfície generalizada na direção $\mathbf{i}$ de ponto $\mathbf{x}$, correspondente a uma força generalizada concentrada unitária aplicada na direção $\mathbf{j}$ do ponto $\xi$.

Assim, a eq.(2.38) representa os deslocamentos dos pontos $\xi$ do domínio $\Omega$, em função dos valores dos deslocamentos e das forças de superfície para pontos $\mathbf{x}$ do 
contorno, quando a placa está submetida ao campo de momentos iniciais, $\mathbf{M}_{\alpha \beta}^{\mathbf{0}}$, à carga distribuída, $\mathbf{q}(\mathbf{x})$, e aos momentos distribuídos, $\mathbf{m}_{\boldsymbol{\alpha}}$, aplicados ao domínio $\boldsymbol{\Omega}$.

\subsubsection{Equações integrais para deslocamentos em pontos do contorno}

Seja a fig.2.2, onde se apresenta um ponto $\xi$, inicialmente no contorno da placa. Acrescentando-se um contorno em arco de circulo, $\Gamma_{\varepsilon}$ com centro em $\xi$ e raio $\boldsymbol{\varepsilon}$, aquele ponto torna-se pertencente ao domínio e a eq.(2.38) pode ser aplicada. Para tanto, o contorno $\Gamma$ é acrescido de um contorno em arco de circulo $\Gamma_{\varepsilon}$ e suprimido da parcela $\bar{\Gamma}$.
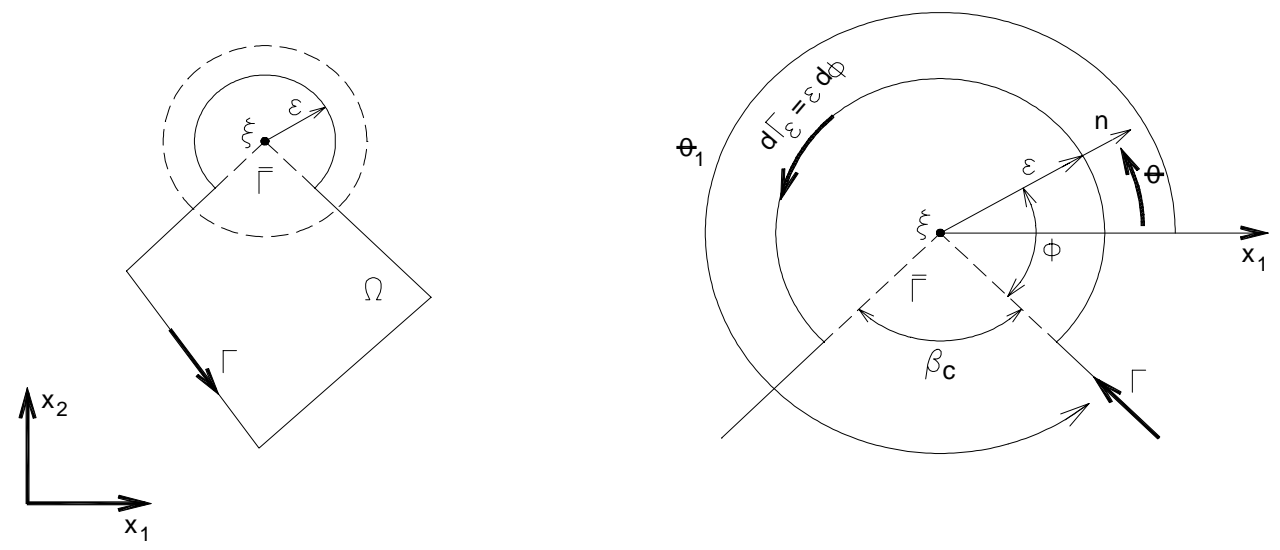

FIGURA 2.2. Placa com alteração de contorno

No contorno $\Gamma_{\varepsilon}$, o ângulo $\phi$ varia de zero a $\left(2 \pi-\beta_{c}\right)$, enquanto o ângulo $\theta$ varia de $\theta_{1}$ a $\left(\theta_{1}-\beta_{c}\right)$.

Assim, pela aplicação da eq.(2.38) e pelo estudo de cada parcela na situação limite, quando o raio $\boldsymbol{\varepsilon}$ tende a zero, chega-se à seguinte equação para deslocamentos nos pontos do contorno, conforme RIBEIRO (1992): 


$$
\begin{aligned}
& \mathbf{C}_{\mathrm{ij}}(\xi) \mathbf{u}_{\mathrm{j}}(\xi)=\int_{\Gamma}\left[\mathbf{u}_{\mathrm{ij}}^{*}(\xi, \mathbf{x}) \mathbf{p}_{\mathbf{j}}(\mathbf{x})-\mathbf{p}_{\mathrm{ij}}^{*}(\xi, \mathbf{x}) \mathbf{u}_{\mathbf{j}}(\mathbf{x})\right] \mathrm{d} \Gamma(\mathbf{x})+ \\
& +\int_{\Omega_{\mathrm{q}}} \mathbf{q}(\mathbf{x})\left[\mathbf{u}_{\mathrm{i} 3}^{*}(\xi, \mathbf{x})-\frac{\mathrm{v}}{(1-v) \lambda^{2}} \mathbf{u}_{\mathrm{i} \alpha, \alpha}^{*}(\xi, \mathbf{x})\right] \mathrm{d} \Omega_{\mathrm{q}}(\mathbf{x})+ \\
& \quad+\int_{\Omega} \mathbf{u}_{\mathrm{i} \alpha, \beta}^{*}(\xi, \mathbf{x}) \mathbf{M}_{\alpha \beta}^{\mathbf{o}}(\mathbf{x}) \mathrm{d} \Omega(\mathbf{x})+\int_{\Omega_{\mathrm{m}}} \mathbf{u}_{\mathrm{i} \alpha}^{*}(\xi, x) \mathbf{m}_{\alpha}(\mathbf{x}) \mathrm{d} \Omega_{\mathrm{m}}(\mathbf{x})
\end{aligned}
$$

Os valores de $\mathbf{C}_{\mathbf{i j}}$ são dados na eq.(2.23), para alguns casos específicos. Para uma situação geral, veja-se RIBEIRO (1992).

\subsubsection{Equações integrais para esforços nos pontos do domínio}

As resultantes de tensão (momentos fletores, torçores e força cortante) são dadas pelas eqs. (2.26) e (2.32). A representação integral para os deslocamentos generalizados nos ponto internos é dada pela eq.(2.38). Assim, para se obter a equação integral dos esforços nos pontos internos, deve-se aplicar as derivações envolvidas nas eqs.(2.26) ou (2.32) na integral dos deslocamentos, eq.(2.38). Logo, faz-se necessária a derivação das funções $\mathbf{u}_{\alpha \gamma, \theta}^{*}$, sob o sinal de integração, as quais contém singularidades. Para a resolução deste problema adotou-se o procedimento utilizado por RIBEIRO (1992), o qual foi baseado nos trabalhos de MIKHLIN (1962), BUI (1978) e TELLES e BREBBIA (1980). Assim, pode-se escrever que:

Para momentos fletores e torçor:

$$
\begin{aligned}
& \mathbf{M}_{\alpha \beta}(\xi)=\int_{\Gamma} \mathbf{u}_{\alpha \beta \mathrm{k}}^{*}(\xi, x) \mathbf{p}_{\mathbf{k}}(\mathbf{x}) \mathrm{d} \Gamma(\mathbf{x})-\int_{\Gamma} \mathbf{p}_{\alpha \beta \mathrm{k}}^{*}(\xi, x) \mathbf{u}_{\mathbf{k}}(\mathbf{x}) \mathrm{d} \Gamma(\mathbf{x})+ \\
& +\int_{\Omega_{1}} \mathbf{r}_{\alpha \beta}^{*}(\xi, x) q(x) d \Omega(x)+\frac{v}{(1-v) \lambda^{2}} q(x) \delta_{\alpha \beta}+\int_{\Omega_{m}} t_{\alpha \beta \gamma}^{*}(\xi, x) m_{\gamma}(x) d \Omega_{m}(x)+ \\
& +\int_{\Omega} \mathbf{e}_{\alpha \beta \gamma \theta}^{*}(\xi, x) M_{\gamma \theta}^{0}(x) d \Omega(x)+g_{\alpha \beta \gamma \theta}^{*}(\xi) M_{\gamma \theta}^{o}(\xi)-M_{\alpha \beta}^{o}(\xi)
\end{aligned}
$$

A expressão $\int_{\Omega} \mathbf{e}_{\alpha \beta \gamma \theta}^{*}(\xi, \mathbf{x}) \mathbf{M}_{\gamma \theta}^{\mathbf{o}}(\mathbf{x}) \mathbf{d} \Omega(\mathbf{x})+\mathbf{g}_{\alpha \beta \gamma \theta}^{*}(\xi) \mathbf{M}_{\gamma \theta}^{\mathbf{o}}(\xi)$ contida na eq. (2.40) originou-se da derivação do termo $\int_{\Omega} \mathbf{u}_{\mathbf{i} \alpha, \beta}^{*}(\xi, \mathbf{x}) \mathbf{M}_{\mathbf{\alpha} \boldsymbol{\beta}}^{\mathbf{o}}(\mathbf{x}) \mathbf{d} \boldsymbol{\Omega}(\mathbf{x})$ da eq.(2.38) em relação às coordenadas do ponto fonte $\xi$. 
Para esforços cortantes:

$$
\begin{aligned}
& \mathbf{Q}_{3 \beta}(\xi)=\int_{\Gamma} \mathbf{u}_{3 \beta \mathbf{k}}^{*}(\xi, x) \mathbf{p}_{\mathbf{k}}(x) \mathrm{d} \Gamma(x)-\int_{\Gamma} \mathbf{p}_{3 \beta \mathbf{k}}^{*}(\xi, x) \mathbf{u}_{\mathbf{k}}(x) \mathrm{d} \Gamma(x)+ \\
& +\int_{\Omega_{\mathrm{q}}} \mathbf{r}_{3 \beta}^{*}(\xi, x) q(x) d \Omega(x)+\int_{\Omega_{\mathrm{m}}} \mathbf{t}_{3 \beta \gamma}^{*}(\xi, x) \mathbf{m}_{\gamma}(x) d \Omega_{\mathbf{m}}(x)+ \\
& +\int_{\Omega} \mathbf{e}_{3 \beta \gamma \theta}^{*}(\xi, x) M_{\gamma \theta}^{o}(x) d \Omega(x)
\end{aligned}
$$

A expressão $\int_{\Omega} \mathbf{e}_{3 \beta \gamma \theta}^{*}(\xi, x) \mathbf{M}_{\gamma \theta}^{\mathbf{o}}(\mathbf{x}) \mathbf{d} \Omega(\mathbf{x})$ que aparece na eq. (2.41) surgiu em função da derivação do termo $\int_{\Omega} \mathbf{u}_{\mathbf{i} \alpha, \beta}^{*}(\xi, \mathbf{x}) \mathbf{M}_{\alpha \beta}^{\mathbf{o}}(\mathbf{x}) \mathbf{d} \Omega(\mathbf{x})$ da eq.(2.38) em relação às coordenadas do ponto fonte $\xi$ e substituída na eq. (2.26.b).

O estudo detalhado das soluções fundamentais contidas na formulação ora apresentada pode ser encontrado em WEEËN (1982), RIBEIRO (1992) e SILVA (1996). As expressões destas soluções estão explicitadas no Apêndice A.

\subsubsection{Transformação das Integrais das cargas e momentos distribuídos no Domínio em Integrais de Contorno}

Considerando-se as equações de deslocamentos (2.38) e (2.39) observa-se que os efeitos das cargas e momentos distribuídos, $\mathbf{q}(\mathbf{x})$ e $\mathbf{m}_{\boldsymbol{\alpha}}(\mathbf{x})$, são calculados através de integrais sobre os subdomínios $\boldsymbol{\Omega}_{\mathbf{q}}$ e $\boldsymbol{\Omega}_{\mathbf{m}}$, respectivamente. Estas integrais deverão ser transformadas em integrais de contorno.

Utilizando-se as transformações apresentadas por WEEËN (1982), a integral da carga distribuída pode ser transformada numa integral sobre o contorno $\Gamma_{\mathbf{q}}$. Segundo o mesmo autor, admitindo-se a carga q(x) uniformemente distribuída, podese demonstrar que para a representação integral dos deslocamentos tem-se:

$$
\begin{aligned}
& \int_{\Omega_{q}}\left[u_{i 3}^{*}(\xi, x)-\frac{v}{(1-v) \lambda^{2}} u_{i \alpha, \alpha}^{*}(\xi, x)\right] q(x) d \Omega(x)= \\
& =q(x) \int_{\Gamma_{q}}\left[v_{i, \alpha}^{*}(\xi, x)-\frac{v}{(1-v) \lambda^{2}} u_{i \alpha}^{*}(\xi, x)\right] n_{\alpha} d \Gamma_{q}(x)
\end{aligned}
$$


A influência do carregamento uniformemente distribuído, q(x), no cálculo dos esforços nos pontos internos, que aparecem nas eqs.(2.40) e (2.41), transformada na integral sobre o contorno $\Gamma_{\mathbf{q}}$, é dada por:

para momentos:

$$
\int_{\Gamma_{\mathbf{q}}} \mathbf{w}_{\alpha \beta}^{*}(\xi, x) q(x) d \Gamma_{q}(x)
$$

para esforços cortantes:

$$
\int_{\Gamma_{q}} w_{3 \beta}^{*}(\xi, x) q(x) d \Gamma_{q}(x)
$$

A carga distribuída pode atuar sobre subdomínios retangulares, estreitos e alongados, conforme mostra a fig.2.3. Neste caso, para efeito de análise numérica, é conveniente considerar uma distribuição de carga equivalente, sobre a linha média do subdomínio.

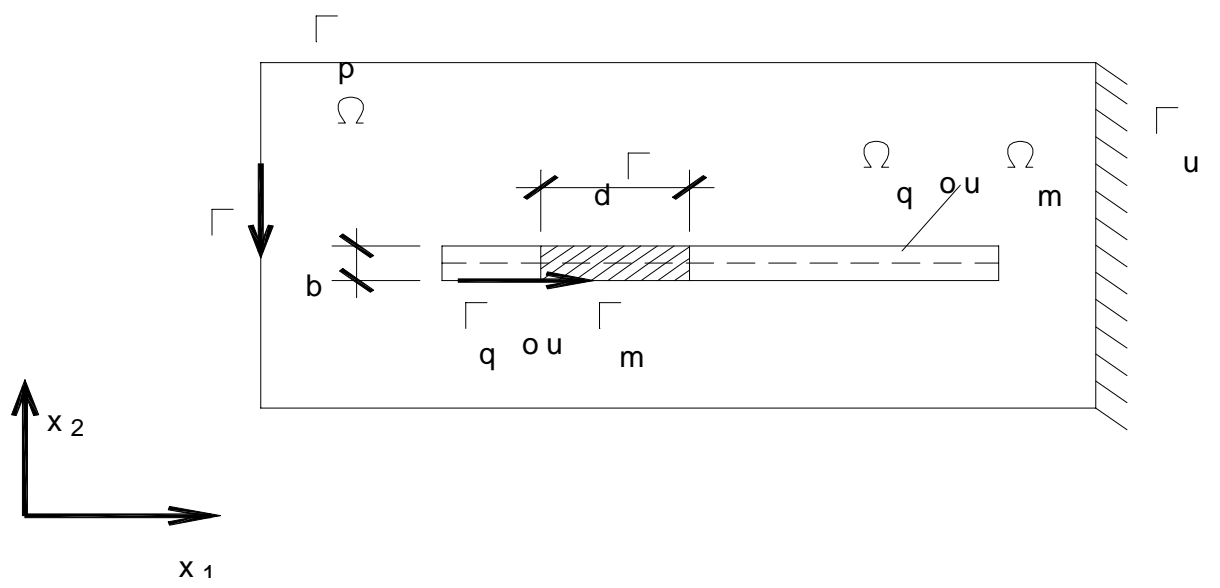

FIGURA 2.3. Cargas ou momentos distribuídos sobre domínios estreitos e alongados.

Para tal, considera-se que a carga distribuída, q, seja constante ao longo da largura $\mathbf{b}$.

Então: 


$$
\begin{aligned}
& \mathrm{d} \Omega_{\mathrm{q}}=\mathbf{b} \cdot \mathrm{d} \Gamma_{\mathrm{q}} \\
& \bar{q}=\mathbf{b} \cdot \mathbf{q}
\end{aligned}
$$

Desta forma, a parcela das equações integrais (2.38) e (2.39) referentes ao carregamento distribuído e expressa por:

$$
\mathbf{I}_{\mathrm{i}}=\int_{\Omega_{\mathrm{q}}}\left[\mathbf{u}_{\mathrm{i} 3}^{*}(\xi, x)-\frac{v}{(1-v) \lambda^{2}} \mathbf{u}_{\mathrm{i} \alpha, \alpha}^{*}(\xi, x)\right] \mathbf{q}(\mathbf{x}) \mathbf{d} \Omega_{\mathrm{q}}(\mathbf{x})
$$

pode ser escrita da seguinte forma:

$$
\mathbf{I}_{\mathrm{i}}=\int_{\Gamma_{\mathrm{q}}}\left[\mathbf{u}_{\mathrm{i} 3}^{*}(\xi, \mathbf{x})-\frac{\mathbf{v}}{(1-v) \lambda^{2}} \mathbf{u}_{\mathrm{i} \alpha, \alpha}^{*}(\xi, x)\right] \overline{\mathbf{q}}(\mathbf{x}) \mathbf{d} \Gamma_{\mathrm{q}}(\mathbf{x})
$$

A influência da carga distribuída em linha $\bar{q}$ nas expressões dos esforços internos, eqs.(2.40) e (2.41), é dada na eq.(2.43) e pode ser escrita da seguinte forma:

$$
\mathbf{I}_{\mathrm{i} \alpha}=\int_{\Gamma_{\mathrm{q}}} \mathbf{r}_{\mathrm{i} \alpha}^{*}(\xi, \mathbf{x}) \overline{\mathbf{q}}(\mathbf{x}) \mathbf{d} \Gamma_{\mathbf{q}}(\mathbf{x})
$$

Quando o ponto fonte estiver situado sobre a linha de carga, deve-se estudar a integral (2.47) uma vez que a função $\mathbf{r}_{\mathbf{i} \alpha}^{*}(\boldsymbol{\xi}, \mathbf{x})$ contida no integrando apresenta singularidades quando o raio tende para zero. Neste caso, a linha de carga é alterada como mostrado na fig. 2.4 . 


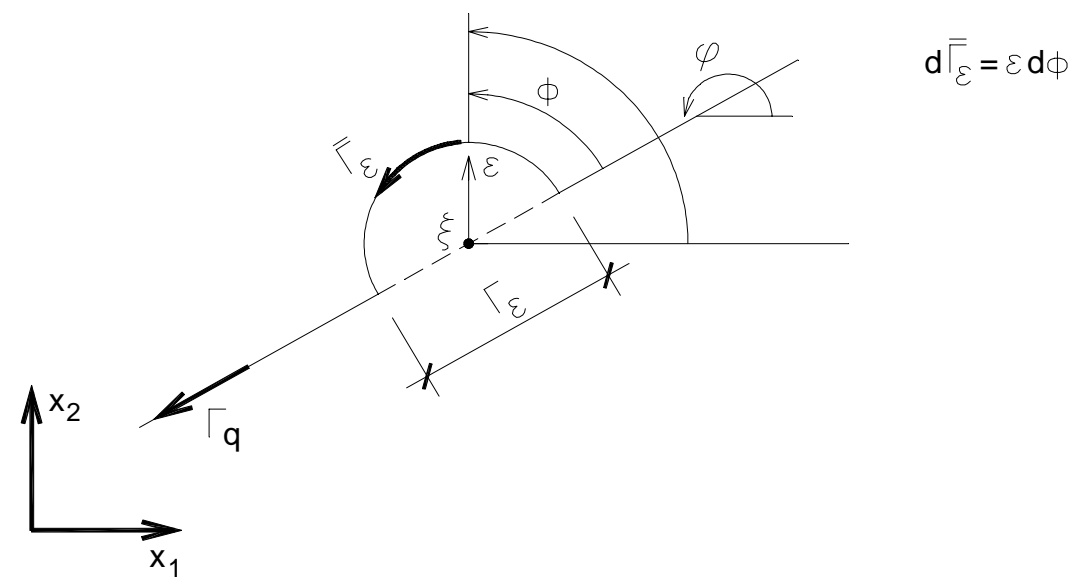

\section{FIGURA 2.4. Linha de carga alterada}

Reescrevendo-se a integral (2.47) para a linha de carga alterada, estudando-se o limite quando $\boldsymbol{\varepsilon}$ tende a zero, para cada parcela envolvida, conclui-se, segundo RIBEIRO (1992), que a influência do carregamento distribuído em linha no cálculo dos momentos, quando o ponto fonte estiver situado sobre a referida linha é dada por:

$$
\int_{\Gamma_{q}} \mathbf{r}_{\alpha \beta}^{*}(\xi, x) \bar{q}(x) d \Gamma_{q}(x)
$$

Procedendo-se de maneira semelhante para os esforços cortantes, a contribuição de $\bar{q}$, quando o ponto fonte $\xi$ estiver situado sobre a linha de carregamento, é determinada por:

$$
\bar{q}(\xi) \frac{n_{\beta}}{\pi}+\int_{\Gamma_{q}} r_{3 \alpha}^{*} \bar{q}(x) d \Gamma_{q}(x)
$$

sendo $\mathbf{n}_{\boldsymbol{\beta}}$ os cossenos diretores do vetor normal ao contorno $\Gamma_{\mathbf{q}}$.

A consideração de momentos $\mathbf{m}_{\mathbf{\alpha}}(\mathbf{x})$, distribuídos num domínio $\boldsymbol{\Omega}_{\mathbf{m}}$, não tem aplicação para os casos práticos que são considerados no presente trabalho. Como é visto mais à frente, na determinação dos esforços e deslocamentos internos, é importante a consideração da aplicação de linhas de momentos. Eis 
porque nesta seção é analisada apenas a aplicação de momentos distribuídos em subdomínios estreitos e alongados (fig.2.3).

Pode-se, então, escrever:

$$
\begin{aligned}
& d \Omega_{m}=b \cdot d \Gamma_{m} \\
& \bar{m}_{\alpha}=b \cdot m_{\alpha}
\end{aligned}
$$

A influência dos momentos distribuídos em linha $\overline{\mathbf{m}}_{\boldsymbol{\alpha}}$, nas expressões dos deslocamentos, eqs.(2.38) e (2.39), pode ser escrita como:

$$
I_{I}=\int_{\Gamma_{\mathrm{m}}} \mathbf{u}_{\mathrm{i} \alpha}^{*}(\xi, \mathbf{x}) \overline{\mathbf{m}}_{\alpha}(\mathbf{x}) \mathrm{d} \Gamma_{\mathrm{m}}(\mathbf{x})
$$

Para a determinação dos esforços internos, $\mathbf{M}_{\alpha \beta}$ e $\mathbf{Q}_{\alpha}$, desenvolve-se um estudo análogo àquele feito para a linha de carga $\overline{\mathrm{q}}$. Assim, a contribuição da linha de momentos, $\overline{\mathbf{m}}_{\boldsymbol{\alpha}}$, é considerada tomando-se a parcela correspondente nas eqs.(2.40) e (2.41) e escrevendo-se:

$$
\int_{\Omega_{\mathrm{m}}} \mathrm{t}_{\mathrm{i} \beta \gamma}^{*}(\xi, x) \mathbf{m}_{\gamma}(x) \mathrm{d} \Omega_{\mathrm{m}}(\mathbf{x})=\int_{\Gamma_{\mathrm{m}}} \mathrm{t}_{\mathrm{i} \beta \gamma}^{*}(\xi, x) \overline{\mathbf{m}}_{\gamma}(\mathrm{x}) \mathrm{d} \Gamma_{\mathrm{m}}(\mathbf{x})
$$

No caso do ponto fonte estar situado sobre a linha de momentos, considera-se o contorno alterado, como mostrado na fig.(2.4). A integral do segundo membro da eq.(2.52), para a determinação dos momentos, no limite quando $\varepsilon$ tende a zero, pode ser determinada a partir da equação:

$$
\begin{aligned}
& \lim _{\varepsilon \rightarrow 0} \int_{\Gamma-\Gamma_{\varepsilon}+\mathbf{T}_{\varepsilon}} \mathbf{t}_{\alpha \beta \gamma}^{*}(\xi, x) \overline{\mathbf{m}}_{\gamma}(\mathrm{x}) \mathrm{d} \Gamma(\mathrm{x})=\lim _{\varepsilon \rightarrow 0} \int_{\Gamma-\Gamma_{\varepsilon}} \mathrm{t}_{\alpha \beta \gamma}^{*}(\xi, x) \overline{\mathbf{m}}_{\gamma}(\mathrm{x}) \mathrm{d} \Gamma(\mathrm{x})+ \\
& +\lim _{\varepsilon \rightarrow 0} \int_{\Gamma_{\varepsilon}} t_{\alpha \beta \gamma}^{*}(\xi, x) \bar{m}_{\gamma}(x) d \bar{\Gamma}_{\varepsilon}(x)
\end{aligned}
$$

A primeira parcela do segundo membro da eq. (2.53) é calculada no sentido do valor principal de Cauchy, admitida a hipótese de que $\overline{\mathbf{m}}_{\gamma}(\mathbf{x})$ satisfaça a condição de Hölder. Do estudo da segunda parcela do segundo membro da eq.(2.53), pode-se escrever:

$$
\begin{aligned}
& \overline{\mathbf{m}}_{\gamma}(\xi) \lim _{\varepsilon \rightarrow 0} \int_{\Gamma_{\varepsilon}} t_{\alpha \beta \gamma}^{*}(\xi, x) d \bar{\Gamma}_{\varepsilon}(x)=\bar{m}_{\gamma}(\xi) \lim _{\varepsilon \rightarrow 0} \frac{1}{4 \pi \varepsilon} \int_{0}^{\pi}[(1-v) \\
& \left.\left(\mathbf{r}_{, \alpha} \delta_{\beta \gamma}+\mathbf{r}_{, \beta} \delta_{\alpha \gamma}-r_{, \gamma} \delta_{\alpha \beta}\right)+2(1+v) r_{, \alpha} \mathbf{r}_{, \beta} \mathbf{r}_{, \gamma}\right] \varepsilon d \phi
\end{aligned}
$$


Os valores explícitos dos limites dados na eq. (2.54) são dados pelas eqs.(a.11) do apêndice A.

A contribuição da linha de momentos distribuídos $\overline{\mathrm{m}}_{\gamma}$ no cálculo das forças cortantes, para pontos situados sobre tal linha, é desenvolvida semelhantemente ao que foi feito para os momentos. Assim, pode-se escrever:

$$
\int_{\Omega_{\mathrm{m}}} \mathrm{t}_{3 \beta \gamma}^{*}(\xi, x) \mathbf{m}_{\gamma}(x) d \Omega_{m}(x)=\int_{\Gamma_{\mathrm{m}}} \mathrm{t}_{3 \beta \gamma}^{*}(\xi, x) \bar{m}_{\gamma}(x) d \Gamma_{m}(x)
$$

Seja o caso do ponto fonte situar-se sobre a linha de momentos. Considere-se o contorno alterado (fig.2.4) e a integral do segundo membro da eq.(2.55); estudando-se o limite quando $\boldsymbol{\varepsilon}$ tende a zero, pode-se escrever:

$$
\begin{aligned}
& \lim _{\varepsilon \rightarrow 0} \int_{\Gamma-\Gamma_{\varepsilon}+\mathbf{\Gamma}_{\varepsilon}} \mathbf{t}_{3 \beta \gamma}^{*}(\xi, x) \overline{\mathbf{m}}_{\gamma}(\mathbf{x}) \mathrm{d} \Gamma(\mathbf{x})=\lim _{\varepsilon \rightarrow 0} \int_{\Gamma-\Gamma_{\varepsilon}} \mathbf{t}_{3 \beta \gamma}^{*}(\xi, x) \overline{\mathbf{m}}_{\gamma}(\mathbf{x}) \mathrm{d} \Gamma(\mathbf{x})+ \\
& +\lim _{\varepsilon \rightarrow 0} \int_{\Gamma_{\varepsilon}} \mathbf{t}_{3 \beta \gamma}^{*}(\xi, x) \overline{\mathbf{m}}_{\gamma}(\mathbf{x}) \mathrm{d} \Gamma(\mathbf{x})
\end{aligned}
$$

Pode-se demonstrar que a contribuição dos momentos em linha no cálculo das forças cortantes é dada apenas pela primeira parcela do segundo membro da eq.(2.56), a qual é interpretada no sentido do valor principal de Cauchy.

\subsubsection{Influência de cargas concentradas}

O termo de domínio dado pela eq. (2.45) pode representar também a influência de cargas concentradas. Desta forma, $\mathbf{I}_{\mathbf{i}}$ transforma-se em:

$$
I_{i}(\xi)=P\left[u_{i 3}^{*}(\xi, x)-\frac{v}{(1-v) \lambda^{2}} u_{i \alpha, \alpha}^{*}(\xi, x)\right]
$$

A expressão (2.57) representa a influência da carga concentrada $\mathbf{P}$ aplicada em $\mathbf{x}$, na equação integral de deslocamentos, para o ponto $\xi$. A influência da carga concentrada no cálculo dos esforços internos $\mathbf{M}_{\alpha \beta}$ e $\mathbf{Q}_{\alpha}$, no ponto $\xi$ é dada diretamente, sem integração, pelas expressões de $\mathbf{r}_{\alpha \beta}^{*}$ e $\mathbf{r}_{\mathbf{3} \beta}^{*}$, respectivamente. Ou seja: 
Para momentos:

$$
\mathbf{I}_{\alpha \beta}=\mathbf{P} \mathbf{r}_{\alpha \beta}^{*}
$$

Para esforço cortante:

$$
\mathbf{I}_{3 \beta}=\mathbf{P} \mathbf{r}_{3 \beta}^{*}
$$

\subsection{DISCRETIZAÇÃO DAS EQUAÇÕES INTEGRAIS}

\subsubsection{Considerações preliminares}

As contribuições das integrações sobre o contorno, dadas no item 2.3, podem ser avaliadas sobre partes (ou elementos) daquele contorno e somadas subseqüentemente. Esta discretização do processo de integração é exata. Todavia, em função das dificuldades de integração analítica destas partes, o contorno da placa é aproximado por uma série de elementos de contorno de geometria pré-estabelecida. No presente trabalho são utilizados elementos de contorno retos. A geometria destes elementos é definida por dois pontos (nós) situados sobre o contorno real da placa. Para facilitar a integração numérica, as coordenadas de cada ponto do elemento são parametrizadas em função das coordenadas dos nós extremos, pelas coordenadas locais homogêneas $\zeta$ (fig.2.5).

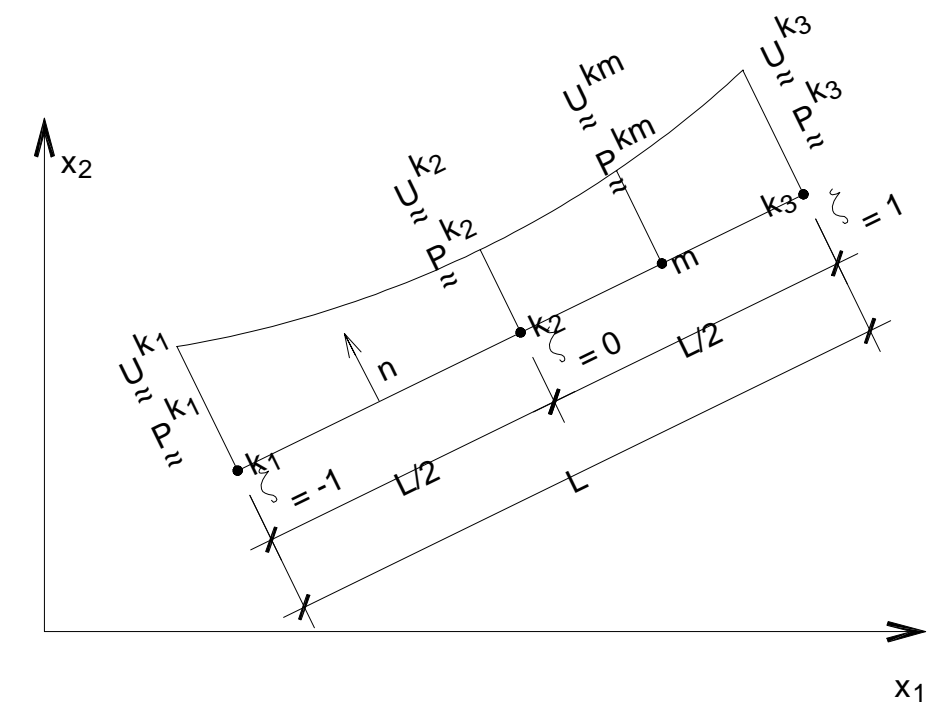

FIGURA 2.5. Elemento de contorno $\Gamma_{\mathbf{j}}$ e aproximação das variáveis do contorno. 
Os valores de deslocamentos e forças de superfícies são aproximados sobre os elementos por funções de interpolação quadráticas, em função dos respectivos valores nodais das extremidades (nós $\mathbf{k}_{\mathbf{1}}$ e $\mathbf{k}_{\mathbf{3}}$ ) e do nó intermediário $\left(\mathbf{k}_{\mathbf{2}}\right)$.

\subsubsection{Deslocamentos nos pontos do contorno}

Mudando-se a variável de integração de $\Gamma$ para $\zeta$ e transcrevendo-se $\mathbf{u}_{\mathbf{j}}$ e $\mathbf{p}_{\mathbf{j}}$ em função dos valores nodais dos deslocamentos e forças de superfície, pode-se escrever a eq.(2.39) como:

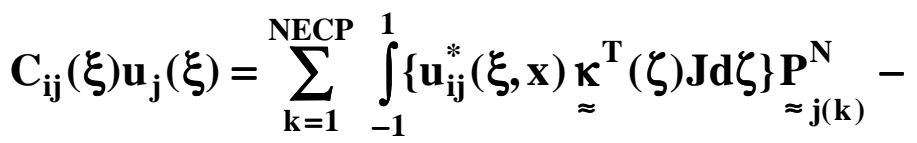

$$
\begin{aligned}
& +\sum_{k=1}^{\operatorname{NECP}} \int_{-1}^{1}\left\{p_{i j}^{*}(\xi, x) \underset{\approx}{\kappa^{T}}(\zeta) J d \zeta\right\} \underset{\approx j(k)}{\mathbf{U}^{N}}+\int_{\Omega_{q}} \mathbf{q}(x)\left[u_{i 3}^{*}(\xi, x)-\right. \\
& \left.+\frac{v}{(1-v) \lambda^{2}} \mathbf{u}_{i \alpha, \alpha}^{*}(\xi, x)\right] d \Omega_{q}(x)+\int_{\Omega} u_{i \alpha, \beta}^{*}(\xi, x) M_{\alpha \beta}^{o}(x) d \Omega(x)+ \\
& +\int_{\Omega_{\mathrm{m}}} \mathbf{u}_{\mathrm{i} \alpha}^{*}(\xi, x) \mathbf{m}_{\alpha}(\mathbf{x}) \mathrm{d} \Omega(\mathbf{x})
\end{aligned}
$$

Onde NECP é o número de elementos de contorno da placa e $\mathbf{J}$ o jacobiano de transformação das variáveis dado por:

$$
J=\frac{d \Gamma}{d \zeta}=\frac{L_{k}}{2}
$$

uma vez que a relação entre as variáveis $\Gamma$ e $\zeta$ é dada por:

$$
\Gamma=\frac{\mathbf{L}}{2} \zeta
$$

A eq.(2.59), quando escrita para todos os pontos nodais do contorno (NNCP), representa um sistema de 3(NNCP) equações algébricas lineares, onde as incógnitas são os valores nodais de deslocamentos $\mathbf{U}_{\mathbf{J}(\mathbf{k})}^{\mathbf{N}}$ ou forças de superfície $\mathbf{P}_{\mathbf{j}(\mathbf{k})}^{\mathbf{N}}$. Observe-se que estes valores nodais foram postos fora das integrais em (2.59), uma vez que não são função da variável de integração $\zeta$. Estas integrações envolvem o produto de soluções fundamentais, funções interpoladoras e jacobianos. As soluções 
fundamentais são funções de $\mathbf{r}$, que depende da posição do ponto $\boldsymbol{\xi}$, agora função de $\zeta$. Dessa forma, pode-se efetuar a integração numérica a qual é simples e eficiente para o tratamento computacional.

As integrais sobre os elementos traduzem a influência dos valores nodais de deslocamentos e forças no contorno, sobre os deslocamentos nos pontos fonte $\xi$. Estas integrais podem ser escritas para cada um dos três pontos nodais do elemento $\Gamma_{\mathbf{k}}$ da seguinte forma:

$$
\begin{aligned}
& \mathbf{g}_{\mathrm{ij}}^{\mathbf{n}}(\xi)=\frac{\mathbf{l}_{\mathrm{k}}}{2} \int_{-1}^{1} \mathbf{u}_{\mathrm{ij}}^{*}(\xi, x) \kappa_{\mathrm{n}}(\zeta) \mathrm{d} \zeta \\
& \mathbf{h}_{\mathrm{ij}}^{\mathbf{n}}(\xi)=\frac{\mathbf{l}_{\mathrm{k}}}{2} \int_{-1}^{1} \mathbf{p}_{\mathrm{ij}}^{*}(\xi, x) \kappa_{\mathrm{n}}(\zeta) \mathrm{d} \zeta
\end{aligned}
$$

onde:

$\mathbf{u}_{\mathbf{i j}}^{*}$ e $\mathbf{p}_{\mathbf{i j}}^{*}$ são as soluções fundamentais generalizadas em deslocamentos e forças de superfície; n: representa o nó local do elemento onde se mede a resposta `a carga unitária aplicada; $\xi$ : ponto fonte, onde é aplicada a carga unitária ou para o qual se escrevem as equações; $\mathbf{i}$ : indica qual equação está sendo gerada para o deslocamento no ponto $\boldsymbol{\xi}$. Se $\mathbf{i}=1,2$, são geradas as equações para as rotações $\boldsymbol{\phi}_{\mathbf{n}}$ e $\boldsymbol{\phi}_{\mathbf{s}}$, respectivamente; se $\mathbf{i}=3$, gera-se a equação do deslocamento transversal $\mathbf{w}$. Em outras palavras, $\mathbf{i}$ indica a ação unitária aplicada em $\boldsymbol{\xi}$. j: indica a natureza da resposta medida em $\mathbf{x}$. Se $\mathbf{j}=1$, a resposta fundamental é relativa ao momento $\mathbf{M}_{\mathbf{n}}^{*}$; se $\mathbf{j}=2$, a resposta é relativa ao momento $\mathbf{M}_{\mathbf{n s}}^{*}$; se $\mathbf{j}=3$, a resposta é relativa à força cortante $\mathbf{Q}_{\mathbf{n}}^{*}$

Observe-se que para os nós extremos, os coeficientes dados pela eq.(2.62) recebem a contribuição dos dois elementos adjacentes.

Gerando-se os coeficientes dados pela eq. (2.62) para os três pontos nodais de todos os NECP elementos, endereçando-os e acumulando-os convenientemente, obtêm-se as matrizes $\underset{\approx}{\mathbf{G}}$ e $\underset{\approx}{\mathbf{H}}$, que são as matrizes de influência dos valores nodais do Método dos Elementos de Contorno. A geração dos coeficientes dados pelas eqs. 
(2.62) é feita utilizando-se um esquema de integração numérica através da quadratura de Gauss.

Assim, com a discretização do contorno e a aproximação das variáveis sobre os elementos, utilizando-se a eq.(2.59), obtém-se um sistema com 3(NNCP) equações e 3(NNCP) incógnitas representado matricialmente da seguinte forma:

$$
\underset{\approx \mathbf{C U}}{\mathbf{C}}+\underset{\approx}{\mathbf{H}}=\underset{\approx}{\mathbf{G}} \mathbf{P}+\mathbf{B}+\underset{\approx}{\mathbf{E} \mathbf{M}^{0}}
$$

onde: $\underset{\approx}{\mathbf{H}}$ e $\mathbf{G}$ são as matrizes de influência; $\underset{\approx}{\mathbf{U}}$ e $\underset{\approx}{\mathbf{P}}$ são os vetores dos deslocamentos e forças de superfície, respectivamente, para todos os pontos nodais do contorno; $\mathbf{B}$ é o vetor que contém a influência de todo o carregamento de domínio, exceto os momentos iniciais $\mathbf{M}^{\mathbf{0}}$; $\mathbf{E}$ é a matriz de influência do campo de momentos iniciais, $\mathbf{M}^{\mathbf{0}}$ sobre os valores das incógnitas do contorno; sua determinação é dada no item 2.4.5.

A matriz $\underset{\approx}{\mathbf{C}}$ depende apenas da geometria do contorno em cada ponto fonte considerado e pode ser incorporada à matriz $\underset{\approx}{\hat{H}}$, resultando:

$$
\underset{\approx}{\mathbf{H}} \underset{\approx}{\mathbf{G}} \mathbf{\mathbf { P }}+\underset{\approx}{\mathbf{B}}+\underset{\approx}{\mathbf{E}} \mathbf{M}^{\mathbf{0}}
$$

O sistema de equações (2.64) deve ser reordenado pela troca de colunas entre as matrizes $\mathbf{G}$ e $\underset{\approx}{\mathbf{H}}$, de forma que todas as incógnitas de contorno sejam acumuladas no lado esquerdo da eq.(2.64).

Quando o ponto fonte está localizado sobre o contorno da placa, diz-se que a formulação é singular, haja vista que quando o ponto de deslocamento coincidir com aquele, $\mathbf{r}$ será zero e haverá singularidades na determinação da eq.(2.59). Por outro lado, quando o ponto fonte é colocado fora do domínio, a uma pequena distância do contorno, diz-se que a formulação é regular. Esta é a formulação utilizada no presente trabalho. 
A distância d do ponto fonte ao contorno é dada por:

$$
\mathbf{d}=\alpha \mathbf{L}_{\mathbf{m}}
$$

onde $\boldsymbol{\alpha}$ é um coeficiente positivo e $\mathbf{L}_{\mathbf{m}}$ é o comprimento médio dos elementos adjacentes, caso o ponto fonte esteja próximo do nó extremo, ou o próprio comprimento do elemento, caso o ponto fonte esteja próximo do nó intermediário, conforme ilustra a fig. 2.6.

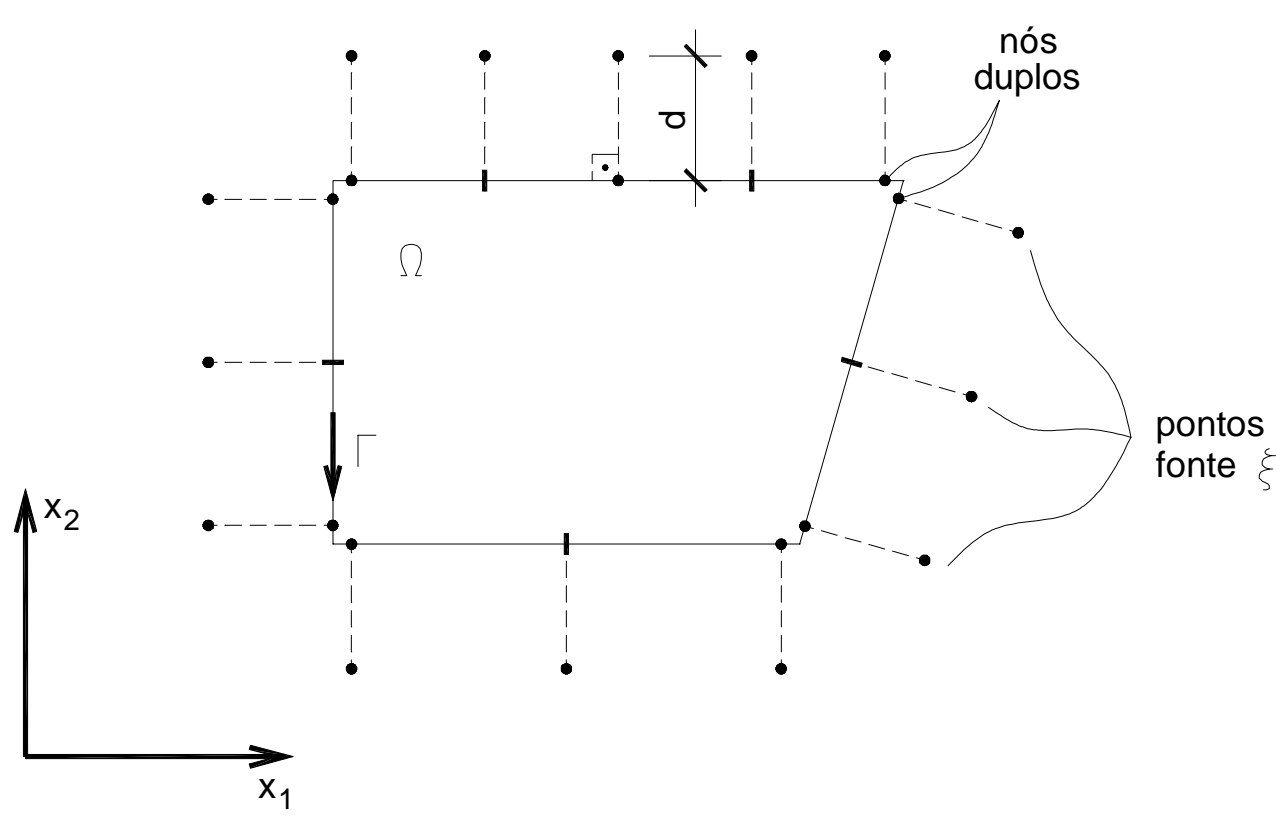

FIGURA 2.6. Pontos fonte $\xi$ colocados fora do domínio da placa

As descontinuidades no contorno decorrentes da presença de pontos angulosos, mudança de vinculação e de forças prescritas, são resolvidas no presente trabalho através da adoção de nós duplos. Tais nós, assim denominados por possuírem as mesmas coordenadas, tornam independentes os valores nodais, antes e depois do ponto considerado.

Observe-se que ao se colocar o ponto fonte fora do domínio da placa, a matriz $\underset{\approx}{\mathbf{C}}$ na eq.(2.63) torna-se nula. 


\section{a) Influência dos carregamentos de domínio nas incógnitas do contorno}

Discretizando-se o contorno da região carregada em NECC elementos retos de contorno, pode-se escrever a influência da carga $\mathbf{q}$ da seguinte forma:

$$
I_{i}=q(x) \sum_{j=1}^{N E C C} \int_{\Gamma_{q_{j}}}\left[v_{i, \alpha}^{*}(\xi, x)-\frac{v}{(1-v) \lambda^{2}} u_{i \alpha}^{*}(\xi, x)\right] n_{\alpha} d \Gamma_{q}(x)
$$

A influência da carga $\mathbf{q}$, na representação integral dos deslocamentos é obtida integrando-se a eq.(2.66), para todos os NECC elementos do contorno $\Gamma_{\mathrm{q}}$, em relação a cada ponto fonte $\xi$. Como estes pontos foram tirados do domínio, eliminam-se todas as singularidades logarítmicas, mesmo quando o contorno $\Gamma_{\mathbf{q}}$ da área da carga uniformemente distribuída for coincidente com o contorno da placa. Os valores das integrais são endereçados e armazenados convenientemente no vetor $\underset{\approx}{\mathbf{B}}$.

Para carga distribuída em linha, sua influência sobre os valores de contorno é dada pela integral (2.46) que é efetuada no contorno $\Gamma_{\mathbf{q}}$, também discretizado em elementos retos $\Gamma_{\mathbf{q} j}$. Admite-se que a carga $\overline{\mathbf{q}}$ varia na forma de parábola do segundo grau sobre cada elemento. Assim, cada elemento necessitará de três pontos, dois extremos e um intermediário. Os valores de $\overline{\mathbf{q}}(\mathbf{x})$ sobre o elemento $\mathbf{j}$ são dados por:

$$
\overline{\mathbf{q}(x)}=\underset{\approx 2}{\kappa_{2}^{T}} \overline{\bar{q}}_{\approx}^{N}=\left[\begin{array}{lll}
\bar{\kappa}_{1} & \bar{\kappa}_{2} & \bar{\kappa}_{3}
\end{array}\right]\left\{\begin{array}{l}
\bar{q} \\
-k 2 \\
\bar{q} \\
-k 3 \\
q
\end{array}\right\}
$$

onde $\bar{\kappa}_{1}, \overline{\mathbf{K}}_{2}, \overline{\mathbf{K}}_{3}$ são dados por:

$$
\bar{\kappa}_{1}(\zeta)=\frac{1}{2} \zeta(\zeta-1) ; \quad \bar{\kappa}_{2}(\zeta)=(1+\zeta)(1-\zeta) ; \quad \bar{\kappa}_{3}(\zeta)=\frac{1}{2} \zeta(1+\zeta)
$$

$\underset{\approx}{\overline{\mathbf{q}}^{\mathbf{N}}}$ representa o vetor que contém os valores nodais da carga distribuída em linha. $\mathbf{k}_{\mathbf{1}}, \mathbf{k}_{\mathbf{2}}$ e $\mathbf{k}_{\mathbf{3}}$, são os pontos nodais do elemento. Pode-se, então, reescrever a eq.(2.46) da seguinte forma:

$$
I_{i}=\sum_{j=1}^{N E L C} \frac{l_{j}}{2} \int_{-1}^{1}\left[u_{i 3}^{*}(\xi, x)-\frac{v}{(1-v) \lambda^{2}} u_{i \alpha, \alpha}^{*}(\xi, x)\right] \underset{\approx 2}{\underset{*}{T} d \zeta \bar{q}^{-N}}
$$


onde NELC é o número de elementos de linha de carga.

Integrando-se numericamente a eq.(2.69) para todos os NELC elementos de linha de carga em relação a todos os pontos do contorno, multiplicando-se pelo vetor $\overline{\mathbf{q}}^{\mathbf{N}}$ correspondente, obtém-se a contribuição da linha de carga no cálculo dos valores de contorno. Tal contribuição é endereçada e armazenada no vetor $\mathbf{B}$.

A contribuição dos momentos distribuídos em linha, no cálculo das incógnitas do contorno, é avaliada de forma semelhante à da carga distribuída em linha. Dividindo-se a linha de distribuição em elementos retos e admitindo-se variação parabólica de $\overline{\mathbf{m}}_{\boldsymbol{\alpha}}$ sobre cada elemento, tem-se:

$$
\bar{m}_{\alpha}(x)=\left\{\begin{array}{l}
\bar{m}_{1}(x) \\
\bar{m}_{2}(x)
\end{array}\right\}=\underset{\approx 3}{\kappa^{T}} \bar{m}_{\alpha}^{N}=\left[\begin{array}{cccccc}
\kappa_{1} & 0 & \kappa_{2} & 0 & \kappa_{3} & 0 \\
0 & \kappa_{1} & 0 & \kappa_{2} & 0 & \kappa_{3}
\end{array}\right]\left\{\begin{array}{l}
\bar{m}_{1} 1 \\
\bar{m}_{2} \\
\bar{m}_{1} 2 \\
\bar{m}_{2} 2 \\
\bar{m}_{1} 3 \\
\bar{m}_{2} 3 \\
\mathrm{~m}_{2}
\end{array}\right\}
$$

$\boldsymbol{\kappa}_{\mathbf{1}}, \boldsymbol{\kappa}_{\mathbf{2}}$, e $\boldsymbol{\kappa}_{\mathbf{3}}$ são dados na eq.(2.68). Os valores $\overline{\mathbf{m}}_{\boldsymbol{\alpha}}^{\mathbf{k}_{\mathbf{i}}}$ representam os valores nodais dos momentos distribuídos $\overline{\mathbf{m}}_{\boldsymbol{\alpha}}$. Com a distretização do contorno $\Gamma_{\mathbf{m}}$ e utilizando-se a expressão de $\overline{\mathbf{m}}_{\boldsymbol{\alpha}}(\mathbf{x})$ dada em (2.70) pode-se escrever a eq.(2.51) como:

$$
I_{i}=\sum_{j=1}^{N E L C} \frac{I_{j}}{2} \int_{-1}^{1} u_{i \alpha}^{*}(\xi, x) \underset{\approx 3}{\kappa^{T}} d \zeta(x) \underset{\approx \alpha}{\bar{m}_{\approx}^{N}}
$$

Integrando-se numericamente a eq.(2.71) para cada elemento de linha de momentos em relação a todos os pontos nodais do contorno, e multiplicando-se pelo vetor nodal correspondente $\underset{\approx \alpha}{\overline{\mathbf{m}}}$, obtém-se a contribuição da linha de momentos no cálculo dos valores de contorno.

Com relação às cargas concentradas, sua contribuição no cálculo dos valores de contorno é feita diretamente com a eq. (2.57). 


\subsubsection{Deslocamentos nos pontos do domínio}

Os deslocamentos nos pontos de domínio expressos pela eq.(2.38) podem ser obtidos diretamente a partir do conhecimento dos valores incógnitos no contorno. A eq.(2.38) é discretizada em função da divisão do contorno da placa em NECP elementos que, escrita para os NPI pontos internos, onde haja interesse na determinação dos deslocamentos, dá origem ao seguinte sistema:

$$
\underset{\approx i}{U}=\underset{\approx i}{G} \underset{\approx}{P}-\underset{\approx i}{H} \underset{\approx}{U}+\underset{\approx i}{B}+\underset{\approx i}{\mathbf{E}} \mathbf{M}^{0}
$$

onde: $\underset{\approx \mathbf{i}}{\mathbf{U}}$ é o vetor de deslocamentos nos pontos do domínio; $\underset{\approx \mathbf{i}}{\mathbf{G}} \underset{\approx \mathbf{i}}{\mathbf{H}}$ são as matrizes de influência para pontos do domínio, obtidas de forma análoga às matrizes $\underset{\approx}{\mathbf{G}}$ e $\underset{\approx}{\mathbf{H}}$ para pontos do contorno; $\underset{\approx \mathbf{i}}{\mathbf{B}}$ é o vetor que contém a influência dos carregamentos de domínio, exceto $\mathbf{M}^{\mathbf{0}}$. Sua obtenção se faz de maneira análoga à do vetor $\underset{\approx}{\mathbf{B}} \underset{\approx \mathbf{i}}{\mathbf{E}}$ é a matriz que exprime a influência dos momentos iniciais $\mathbf{M}^{\mathbf{0}}$, nos deslocamentos dos pontos internos. Sua determinação é dada no item 2.4.5.

Os deslocamentos nos pontos do domínio são obtidos em relação ao sistema de referência global $\mathbf{x}_{\mathbf{1}}, \mathbf{x}_{\mathbf{2}}$ e $\mathbf{x}_{\mathbf{3}}$. Assim, para um ponto interno qualquer $\mathbf{k}$, tem-se:

$$
\left.\underset{\approx \mathrm{i}}{\left[\mathbf{U}^{\mathrm{k}}\right.}\right]^{\mathrm{T}}=\left\{\begin{array}{lll}
\phi_{\mathrm{x}_{1}}^{\mathrm{k}} & \phi_{\mathrm{x}_{2}}^{\mathrm{k}} & \mathbf{w}^{\mathrm{k}}
\end{array}\right\}
$$

Na eq.(2.72), os coeficientes de $\underset{\approx \mathbf{i}}{\mathbf{G}} \underset{\approx \mathbf{i}}{\mathbf{H}}$ são determinados numericamente, sem ocorrência de singularidades, uma vez que $\xi$ é interno e $\mathbf{x}$ pertence ao contorno. O mesmo não acontece na determinação do vetor $\underset{\approx \mathbf{i}}{\mathbf{B}}$; quando o ponto interno $\xi$, para o qual são escritas as equações dos deslocamentos, coincide com a linha de carga, linha de momentos ou com a carga concentrada, surgirão singularidades.

Caso haja carga concentrada $\mathbf{P}$, quando o ponto $\xi$ coincidir com o ponto de aplicação da carga, a contribuição para os coeficientes $\mathbf{B}_{\mathbf{i}}$, na eq.(2.72), é obtida através da expressão dada na eq.(2.57). Analisando-se as soluções fundamentais envolvidas nesta expressão, vê-se que na situação limite, quando $\mathbf{r}$ tende a zero, pode-se escrever: 


$$
\lim _{\mathbf{r} \rightarrow 0} \mathbf{P}\left[\mathbf{u}_{\mathrm{i} 3}^{*}-\frac{v}{(1-v) \lambda^{2}} \mathbf{u}_{\mathrm{i} \alpha, \alpha}^{*}\right]=\mathbf{0}
$$

Na ocorrência de cargas e/ou momentos distribuídos em linha, o ponto fonte $\xi$ pode coincidir com um dos três pontos nodais do elemento de carga. Cada uma destas situações é analisada em SILVA (1996). O mesmo procedimento é adotado no presente trabalho.

\subsubsection{Esforços nos pontos do domínio}

Na determinação dos momentos e forças cortantes para os pontos do domínio onde haja interesse, utilizam-se as equações (2.40) e (2.41), respectivamente. Nestas equações percebe-se que os esforços são dados em função dos valores dos deslocamentos e forças de superfície para os pontos do contorno $\Gamma$ da placa e dos carregamentos de domínio. Estas equações são discretizadas segundo o raciocínio contido nos itens 2.4.1. e 2.4.2., e escritas para todos os NPI pontos internos sendo, então, representadas matricialmente da seguinte forma:

$$
\begin{aligned}
& \left.\underset{\approx}{\mathbf{M}}=\underset{\approx}{\mathbf{G}} \mathbf{\sim} \mathbf{\sim}-\underset{\approx}{\mathbf{H}^{\prime}} \underset{\approx}{\mathbf{U}}+\underset{\approx}{\mathbf{B}^{\prime}}+\underset{\approx}{\left(\mathbf{E}^{\prime}\right.}-\underset{\approx}{\mathbf{I}}\right) \mathbf{M}^{\mathbf{0}} \\
& \underset{\approx}{\mathbf{Q}} \underset{\approx}{\mathbf{G}} \underset{\approx}{\mathbf{P}}-\underset{\approx}{\mathbf{H}} \underset{\approx}{\mathbf{U}}+\underset{\approx}{\mathbf{B}}+\underset{\approx}{\mathbf{E}} \underset{\approx}{\mathbf{M}^{0}}
\end{aligned}
$$

onde:

$\underset{\approx}{\mathbf{M}, \mathbf{Q}}$ são os vetores dos momentos e forças cortantes, respectivamente; $\underset{\approx}{\mathbf{G}}, \underset{\approx}{\mathbf{H}}$,

$\underset{\approx}{\mathbf{G}} \underset{\approx}{\mathbf{H}}$ " são matrizes de influência; $\underset{\approx}{\mathbf{P}} \underset{\approx}{\mathbf{U}}$ são os vetores dos valores nodais de forças de superfície e deslocamentos; $\underset{\approx}{\mathbf{B}}, \underset{\approx}{\mathbf{B}}$ ” são os vetores de influência do carregamento de domínio, exceto $\mathbf{M}^{\mathbf{0}}$, sobre os momentos e forças cortantes, respectivamente; $\underset{\approx}{\mathbf{E}}, \underset{\approx}{\mathbf{E}}$ ” são as matrizes que exprimem a influência dos momentos iniciais sobre os momentos e forças cortantes, respectivamente; $\underset{\approx}{\mathbf{I}}$ é a matriz identidade; $\mathbf{M}^{\mathbf{0}}$ vetor dos momentos iniciais.

O cálculo das matrizes $\mathbf{E}$ ' e E” é detalhado no item 2.4.5. 
A influência dos diversos tipos de carregamento na determinação dos esforços é estudada a seguir.

a) influência da carga distribuída

A influência da carga distribuída $\mathbf{q}(\mathbf{x})$, atuando no domínio $\boldsymbol{\Omega}_{\mathbf{q}}$, no cálculo dos esforços nos pontos do domínio deve ser transformada numa integral sobre o contorno $\Gamma_{\mathbf{q}}$. Considerando-se este contorno discretizado em elementos retos, segundo os itens 2.4.1 e 2.4.2, e carga $\mathbf{q ( x )}$ uniformemente distribuída, podem-se escrever:

$$
b_{i}^{\prime}(\xi)=q \sum_{j=1}^{N E C C} \frac{L_{j}}{2} \int_{-1}^{1} w_{\alpha \beta}^{*} d \zeta ; \quad b_{\beta}^{\prime \prime}(\xi)=q \sum_{j=1}^{N E C C} \frac{L_{j}}{2} \int_{-1}^{1} w_{3 \beta}^{*} d \zeta
$$

onde NECC é o número de elementos de contorno da região carregada.

Os vetores B' e B” dados nas eqs.(2.75) e (2.76) são formados pelas contribuições dos coeficientes $\mathbf{b}_{\mathbf{i}}^{\prime}$ e $\mathbf{b}_{\boldsymbol{\beta}}$, respectivamente.

b) Influência da carga concentrada

A influência da aplicação de uma carga concentrada $\mathbf{P}$ no ponto $\mathbf{x}$, no cálculo dos esforços no ponto interno $\xi$, é obtida diretamente pelas eqs.(2.58).

No caso em que $\boldsymbol{\xi}=\mathbf{x}$, ou seja, quando o ponto onde se deseja conhecer os esforços, coincidir com o ponto de aplicação da carga $\mathbf{P}$, os valores dados nas eqs. (2.77) são considerados na situação limite, quando a distância $\mathbf{r}$ tende a zero. Assim:

$$
\mathbf{b}_{\mathbf{i}}^{\prime}(\xi)=\mathbf{P} \lim _{\mathbf{r} \rightarrow 0} \mathbf{r}_{\alpha \beta}^{*}=-\frac{v \delta_{\alpha \beta}}{\lambda^{2}} \mathbf{P} ; \quad \mathbf{b}_{\beta}^{\prime}(\xi)=\mathbf{P} \lim _{\mathbf{r} \rightarrow 0} \mathbf{r}_{3 \beta}^{*}=\mathbf{0}
$$

Os valores de $\mathbf{b}_{\mathbf{i}}^{\prime}$ e $\mathbf{b}_{\boldsymbol{\beta}}$ convenientemente endereçados e armazenados, levam a contribuição da carga concentrada $\mathbf{P}$ na formação dos vetores $\underset{\approx}{\mathbf{B}}$, e $\underset{\approx}{\mathbf{B}}$ ”, respectivamente. 
c) Influência da linha de carga

A contribuição da linha de carga distribuída $\overline{\mathbf{q}}(\mathbf{x})$, dada na eq.(2.47), na forma discretizada, fica expressa por:

$$
b_{i}^{\prime}(\xi)=\sum_{j=1}^{N E L C} \frac{L_{j}}{2} \int_{-1}^{1} r_{\alpha \beta}^{*} \underset{\approx 2}{\kappa^{T}} d \underset{\approx}{\bar{q}} ; \quad b_{\beta}^{\prime}(\xi)=\sum_{j=1}^{N E L C} \frac{L_{j}}{2} \int_{-1}^{1} r_{3 \beta}^{*} \underset{\approx 2}{\kappa^{T} d \zeta^{-q^{N}}}
$$

onde $\underset{\approx 2}{\kappa}$ é dado nas eqs.(2.67) e (2.68), e $\underset{\approx}{\overline{\mathbf{q}}^{\mathbf{N}}}$ são os valores nodais da carga $\overline{\mathbf{q}}$.

Os coeficientes dados da eq.(2.79) dão a contribuição da carga distribuída em linha, $\overline{\mathbf{q}}$ no cálculo dos momentos e cortantes nos pontos internos $\xi$, e são endereçados e armazenados convenientemente nos vetores $\underset{\approx}{\mathbf{B}}$ e $\underset{\approx}{\mathbf{B}}$ ”, respectivamente.

Caso o ponto $\boldsymbol{\xi}$ coincida com um dos pontos $\mathbf{k}_{\mathbf{1}}, \mathbf{k}_{\mathbf{2}}$ ou $\mathbf{k}_{\mathbf{3}}$ do elemento $\mathbf{j}$, as integrais dadas nas eqs.(2.79) deverão ser calculadas analiticamente sobre o elemento j, conforme procedimento apresentado em SILVA (1996).

\section{d) Influência da linha de momentos}

A contribuição da linha de momentos $\overline{\mathbf{m}}_{\boldsymbol{\alpha}}(\mathbf{x})$ no cálculo dos esforços nos pontos do domínio, dada na eq.(2.52) e escrita na forma discretizada torna-se:

$$
\begin{aligned}
& \mathbf{b}_{i}^{\prime}(\xi)=\sum_{j=1}^{\operatorname{NECC}} \frac{\mathbf{L}_{j}}{2} \int_{-1}^{1} t_{\alpha \beta \gamma}^{*}(\xi, x) \underset{\approx 3}{\kappa^{T}}(\zeta) d \zeta_{\approx \gamma}^{-\mathbf{m}^{N}} \\
& \text { para momentos, e: } \\
& b_{\beta}^{\prime \prime}(\xi)=\sum_{j=1}^{\operatorname{NECC}} \frac{L_{j}}{2} \int_{-1}^{1} t_{3 \beta \gamma}^{*}(\xi, x) \underset{\approx 3}{\kappa^{T}}(\zeta) d \zeta_{\approx \gamma}^{-m^{N}} \\
& \text { para forças cortantes }
\end{aligned}
$$

onde $\underset{\approx 3}{\mathbf{K}}(\zeta)$ é dada pelas eqs. (2.68) e (2.70) e $\underset{\approx \gamma}{\mathbf{m}_{\boldsymbol{\gamma}}^{\mathbf{N}}}$ são os valores nodais da linha de momentos $\overline{\mathbf{m}}_{\boldsymbol{\gamma}}(\mathbf{x})$. 
Os coeficientes $\mathbf{b}_{\mathbf{i}}^{\prime} \mathbf{e} \mathbf{b}_{\boldsymbol{\beta}}$ dados na eq.(2.80) para os pontos internos $\xi$ são endereçados e armazenados convenientemente nos vetores $\mathbf{B}$ ' e $\mathbf{B}$ ”.

\subsubsection{Influência dos momentos iniciais}

$\mathrm{Na}$ formulação apresentada para as equações integrais considerou-se a possibilidade de ocorrência de um campo de momentos iniciais $\mathbf{M}_{\alpha \beta}^{\mathbf{0}}$, atuante no domínio da placa. Tal campo de momentos, em geral, é conhecido apenas em alguns pontos discretos, fazendo-se necessária a divisão do domínio em subdomínios menores sobre os quais admite-se uma determinada variação para $\mathbf{M}^{\mathbf{0}}{ }_{\alpha \beta}$.

No presente trabalho o domínio da placa é dividido em células triangulares, considerando-se variação linear de $\mathbf{M}^{\mathbf{0}}{ }_{\alpha \beta}$ sobre as mesmas. Cada célula é definida pelos vértices $\mathbf{k}_{\mathbf{1}}, \mathbf{k}_{\mathbf{2}}$ e $\mathbf{k}_{\mathbf{3}}$, onde são conhecidos os valores das componentes de $\mathbf{M}_{\boldsymbol{\alpha} \boldsymbol{\beta}}^{\mathbf{0}}$, conforme ilustra a fig.2.7.

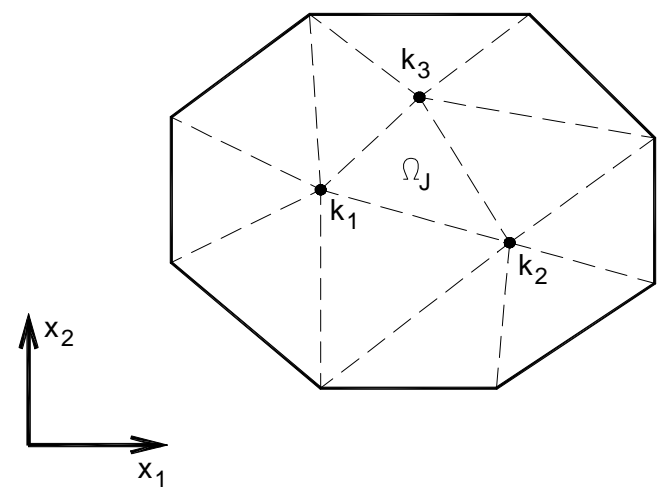

\section{FIGURA 2.7. Divisão do domínio $\Omega$ em células triangulares.}

Na fig.2.8.a apresenta-se uma célula genérica, $\boldsymbol{\Omega}_{\mathbf{j}}$, com variação linear de $\mathbf{M}^{\mathbf{0}}{ }_{\alpha \beta}$.

Dada a necessidade de integração numérica bidimensional para calcular as integrais de domínio sobre as células, as coordenadas de um ponto qualquer de $\boldsymbol{\Omega}_{\mathbf{j}}$ são parametrizadas em função das coordenadas dos vértices, estabelecendo-se sobre a célula um sistema de coordenadas homogêneas, como indica a fig. 2.8.b. 


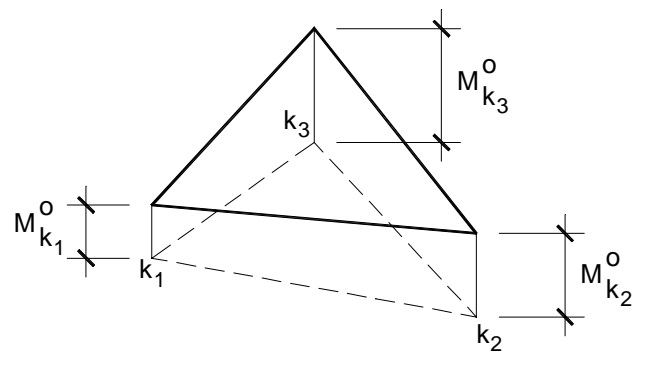

a) Variação linear de $\mathbf{M}^{\mathbf{0}}{ }_{\alpha \beta}$

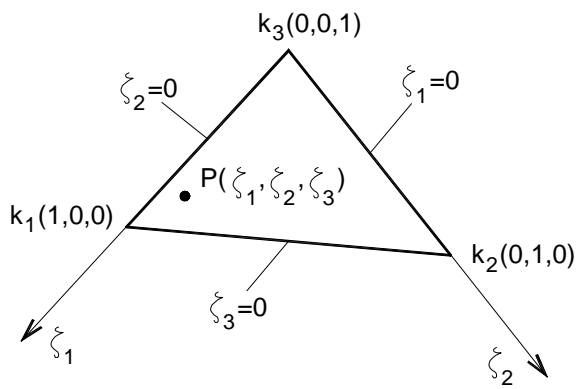

b) Sistema de coordenadas homogêneas

\section{FIGURA 2.8-Célula genérica $\Omega_{\mathbf{j}}$}

As coordenadas cartesianas de um ponto genérico $\mathbf{p}$, pertencente a $\boldsymbol{\Omega}_{\mathbf{j}}$, podem ser expressas por:

onde

$$
\begin{aligned}
& \underset{\approx}{\mathbf{x}^{p}}=\left\{\begin{array}{l}
\mathbf{x}_{1} \\
\mathbf{x}_{2}
\end{array}\right\}^{\mathbf{p}}=\underset{\approx}{\phi^{T}} \underset{\mathbf{X}^{N}}{ } \\
& \underset{\approx}{\phi^{\mathbf{T}}}=\left[\begin{array}{llllll}
\zeta_{1} & 0 & \zeta_{2} & 0 & \zeta_{3} & 0 \\
0 & \zeta_{1} & 0 & \zeta_{2} & 0 & \zeta_{3}
\end{array}\right] \\
& \underset{\approx}{\mathrm{X}^{\mathrm{N}^{\mathrm{T}}}}=\left\{\begin{array}{llllll}
\mathrm{x}_{1}^{\mathrm{k} 1} & \mathbf{x}_{2}^{\mathrm{k} 1} & \mathbf{x}_{1}^{\mathrm{k} 2} & \mathbf{x}_{2}^{\mathrm{k} 2} & \mathbf{x}_{1}^{\mathrm{k3}} & \mathbf{x}_{2}^{\mathrm{k} 3}
\end{array}\right\}
\end{aligned}
$$

ou ainda:

$$
\mathbf{x}_{\mathrm{i}}^{\mathrm{p}}=\zeta_{1}^{\mathrm{p}} \mathbf{x}_{\mathrm{i}}^{\mathrm{k} 1}+\zeta_{2}^{\mathrm{p}} \mathbf{x}_{\mathrm{i}}^{\mathrm{k} 2}+\zeta_{3}^{\mathrm{p}} \mathbf{x}_{\mathrm{i}}^{\mathrm{k} 3}
$$

As coordenadas homogêneas do ponto $\mathbf{p}$, escritas em função das coordenadas cartesianas, ficam:

$$
\zeta_{{ }_{j}}^{p}=\frac{1}{2 A}\left(2 A_{0}^{j}+b^{j} x_{1}^{p}+a^{j} x_{2}^{p}\right)
$$

sendo:

$$
\begin{aligned}
& \mathbf{a}^{\mathrm{j}}=\mathbf{x}_{1}^{\mathrm{l}}-\mathbf{x}_{1}^{\mathrm{k}} ; \quad \mathbf{b}^{\mathrm{j}}=\mathbf{x}_{2}^{\mathrm{k}}-\mathbf{x}_{2}^{\mathrm{l}} \\
& 2 A_{0}^{j}=x_{1}^{k} x_{2}^{l}-x_{1}^{l} x_{2}^{k} ; \quad A=1 / 2\left(b^{1} a^{2}-b^{2} a^{1}\right) \\
& \mathrm{j}=1,2,3 ; \quad \mathrm{k}=2,3,1 ; \quad \mathrm{l}=3,1,2
\end{aligned}
$$

com: 
Aproximando-se o campo de momentos iniciais $\mathbf{M}_{\boldsymbol{\alpha} \boldsymbol{\beta}}^{\mathbf{0}}$ sobre a célula em função dos valores nodais dos vértices, tem-se:

$$
\underset{\approx}{\mathbf{M}^{0^{p}}}=\left\{\begin{array}{l}
M_{1}^{o} \\
M_{2}^{0} \\
M_{3}^{0}
\end{array}\right\}=\underset{\approx}{\underset{\Psi^{T}}{\mathbf{M}^{0^{N}}}}
$$

onde:

$$
\begin{aligned}
& \underset{\Psi^{T}}{\approx}=\left\{\begin{array}{ccccccccc}
\zeta_{1} & 0 & 0 & \zeta_{2} & 0 & 0 & \zeta_{3} & 0 & 0 \\
0 & \zeta_{1} & 0 & 0 & \zeta_{2} & 0 & 0 & \zeta_{3} & 0 \\
0 & 0 & \zeta_{1} & 0 & 0 & \zeta_{2} & 0 & 0 & \zeta_{3}
\end{array}\right\} \\
& \underset{\approx}{\mathbf{M}^{\mathbf{0}^{\mathrm{N}}}}=\left\{M_{1}^{0, \mathrm{k} 1} \mathbf{M}_{2}^{0, \mathrm{k} 1} \mathbf{M}_{3}^{0, \mathrm{k} 1} \mathbf{M}_{1}^{0, \mathrm{k} 2} \mathbf{M}_{2}^{0, \mathrm{k} 2} \mathbf{M}_{3}^{0, \mathrm{k} 2} \mathbf{M}_{1}^{0, \mathrm{k} 3} M_{2}^{0, \mathrm{k} 3} M_{3}^{0, \mathrm{k} 3}\right\}
\end{aligned}
$$

Como visto, a contribuição do campo de momentos iniciais sobre os deslocamentos no contorno, é dada por:

$$
\mathbf{I}_{\mathbf{i}}(\xi)=\int_{\Omega} \mathbf{u}_{\mathrm{i} \alpha, \beta}^{*}(\xi, x) \mathbf{M}_{\alpha \beta}^{\mathbf{0}}(\mathbf{x}) \mathrm{d} \Omega(\mathbf{x})
$$

Assim, com a discretização do domínio em células e a aproximação adotada para $\underset{\approx \alpha \beta}{\mathbf{M}^{\mathbf{0}}}$, pode-se escrever a eq.(2.88) da seguinte forma:

$$
I_{i}(\xi)=\sum_{j=1}^{N C E L} 2 A_{j} \int_{0}^{1}\left[\int_{0}^{1-\zeta_{2}} u_{i \alpha, \beta}^{*}(\xi, x) \underset{\approx}{\Psi} d \zeta_{1}\right] d \underset{\approx}{\Psi_{2}} \underset{\mathbf{M}^{0^{N}}}{\mathbf{s}^{\mathrm{T}}}
$$

onde $\mathbf{A}_{\mathbf{j}}$ é a área da célula, NCEL é o número de células adotado.

A integral sobre cada célula:

$$
\mathbf{e}_{\mathrm{ik}}=2 \mathrm{~A}_{\mathrm{j}} \int_{0}^{1}\left[\int_{0}^{1-\zeta_{2}} \mathbf{u}_{\mathrm{i} \alpha, \beta}^{*}\left(\xi_{,}\right) \underset{\approx}{\Psi^{T}} \mathrm{~d} \zeta_{1}\right] \mathrm{d} \zeta_{2}
$$

é efetuada numericamente, usando-se a quadratura de Gauss para domínio triangular.

A influência de uma célula nos três deslocamentos generalizados de um ponto do contorno, eq.(2.89), é dada pela submatriz e de dimensões (3x9). Esta submatriz 
devidamente calculada é endereçada na matriz global $\underset{\approx}{\mathbf{E}}$. Assim, para um ponto i do contorno e para a célula $\mathbf{j}$ com vértices $\mathbf{k}_{\mathbf{1}}, \mathbf{k}_{\mathbf{2}}$ e $\mathbf{k}_{\mathbf{3}}$, tem-se:

$$
\underset{\approx}{\mathbf{e}}=\left[\left[\begin{array}{c}
\mathbf{e}_{1} \\
\approx
\end{array}\right]\left[\begin{array}{c}
\mathbf{e}_{2} \\
\approx
\end{array}\right]\left[\begin{array}{c}
\mathbf{e}_{3} \\
\approx
\end{array}\right]\right]
$$

Onde as submatrizes $\mathbf{e}_{1}, \mathbf{e}_{2}$ e $\mathbf{e}_{3}$ são de dimensão (3x3). As três colunas da submatriz $\mathbf{e}_{\mathbf{1}}$ representam a influência das três componentes de $\mathbf{M}_{\alpha \beta}^{\mathbf{0}}$ do ponto $\mathbf{k}_{\mathbf{1}}$ sobre os valores de contorno do ponto $\mathbf{i}$; as três colunas de $\mathbf{e}_{2}$ são a influência das componentes de $\mathbf{M}^{\mathbf{0}}{ }_{\alpha \beta}$ do ponto $\mathbf{k}_{2}$ e as três colunas de $\mathbf{e}_{3}$ representam a influência das componentes de $\mathbf{M}_{\boldsymbol{\alpha} \beta}^{\mathbf{0}}$ do ponto $\mathbf{k}_{\mathbf{3}}$, sobre os valores de contorno do ponto $\mathbf{i}$.

A matriz $\underset{\approx}{\mathbf{E}}$ é gerada calculando-se as submatrizes $\underset{\approx}{\mathbf{e}}$ para todas as células em relação a cada ponto do contorno.

A consideração da influência do campo de momentos iniciais $\mathbf{M}_{\boldsymbol{\alpha} \beta}^{\mathbf{0}}$ sobre os valores dos deslocamentos nos pontos internos é feita semelhantemente ao que foi desenvolvido para a obtenção da matriz $\underset{\approx}{\mathbf{E}}$. A matriz gerada é a matriz $\underset{\approx}{\overline{\mathbf{E}}}$.

Os coeficientes das matrizes $\underset{\sim}{\mathbf{E}}$ e $\underset{\sim}{\overline{\mathbf{E}}}$ são calculados usando-se integração numérica de Gauss para domínios triangulares e tabelas de COWPER apud RIBEIRO (1992). Observa-se que nestes casos não não ocorrem singularidades.

Para os valores dos esforços nos pontos internos, a influência do campo de momentos iniciais é dada por:

$$
I_{\alpha \beta}(\xi)=\int_{\Omega} \mathbf{e}_{\alpha \beta \gamma \theta}^{*}(\xi, x) M_{\gamma \theta}^{0}(x) d \Omega(x) ; \quad I_{3 \beta}(\xi)=\int_{\Omega} \mathbf{e}_{3 \beta \gamma \theta}^{*}(\xi, x) M_{\gamma \theta}^{0}(x) d \Omega(x)
$$

para momentos e esforços cortantes, respectivamente.

Dada a divisão do domínio em células e a aproximação adotada para $\mathbf{M}^{\mathbf{0}}{ }_{\boldsymbol{\gamma}}$ segundo a eq. (2.87), estas integrais ficam expressas por:

$$
\begin{aligned}
& I_{\alpha \beta}(\xi)=\sum_{j=1}^{N C E L} \int_{\Omega_{J}} e_{\alpha \beta \gamma \theta}^{*}(\xi, x) \underset{\approx d}{\Psi} \Omega_{j}(x) \underset{\approx}{M^{0^{N}}} \\
& I_{3 \beta}(\xi)=\sum_{j=1}^{N C E L} \int_{\Omega_{J}} e_{3 \beta \gamma \theta}^{*}(\xi, x) \underset{\approx}{\Psi} \Omega_{j}(x) \underset{\approx}{M^{0^{N}}}
\end{aligned}
$$


As integrais dadas nas eqs.(2.91.a,b) são calculadas numericamente, utilizando-se a quadratura de Gauss para domínios triangulares e as tabelas de COWPER, exceto quando o ponto fonte $\xi$ coincidir com um dos vértices da célula; neste caso ocorrem singularidades fortes do tipo $\mathbf{1} / \mathbf{r}$ nas integrais (2.91.a). Utiliza-se, então, integração analítica em $\mathbf{r}$ e integração numérica em $\boldsymbol{\theta}$. A integração analítica é efetuada sobre as expansões polinomiais de $\mathbf{K}_{\mathbf{0}}(\mathbf{z}), \mathbf{K}_{\mathbf{1}}(\mathbf{z})$ e $\mathbf{A}(\mathbf{z})$. Como estas expansões têm expressões distintas para $\mathbf{0} \leq \mathbf{z} \leq \mathbf{2}$ e para $\mathbf{z}>\mathbf{2}$, a célula que contém 0 ponto singular deve ser dividida em três sub-células triangulares, como ilustra a fig.2.9. Assim, a sub-célula adjacente ao nó singular $\xi$ possui os dois lados concorrentes neste ponto com comprimentos iguais a $2 / \lambda$ definindo-se, portanto, dois pontos auxiliares $\mathbf{k}_{\mathbf{4}}$ e $\mathbf{k}_{\mathbf{5}}$. Com este procedimento ficam caracterizadas três células: a primeira, $\mathrm{J}_{\mathbf{1}}$, formada pelos vértices $\mathbf{k}_{\mathbf{1}}, \mathbf{k}_{\mathbf{4}}$ e $\mathbf{k}_{5}$, que contém o ponto singular $\boldsymbol{\xi}$ e duas não singulares, $\mathbf{J}_{\mathbf{2}}$ e $\mathbf{J}_{\mathbf{3}}$ definidas, respectivamente, pelos vértices $\mathbf{k}_{\mathbf{2}}, \mathbf{k}_{\mathbf{3}}, \mathbf{k}_{\mathbf{4}}$ e $\mathbf{k}_{\mathbf{3}}, \mathbf{k}_{\mathbf{5}}$, $\mathbf{k}$.

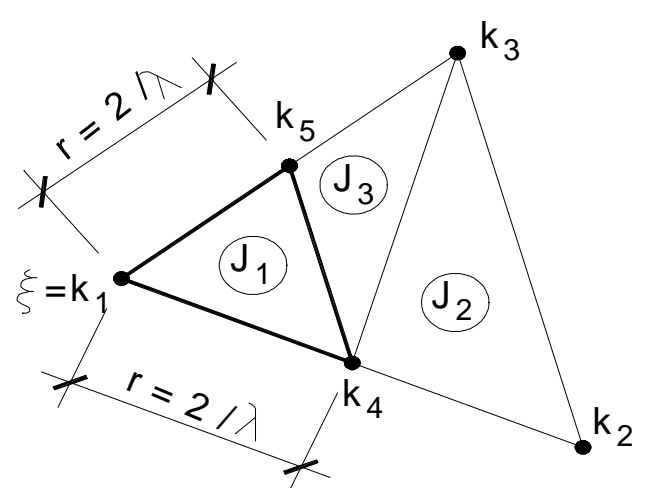

Figura 2.9. Sub-divisão da célula com ponto singular

Para a sub-célula $\mathrm{J}_{1}$, com o nó singular $\xi$, efetua-se primeiramente a integração analítica em $\mathbf{r}(\boldsymbol{\xi}, \mathbf{x})$ e em seguida efetua-se a integração numérica em $\boldsymbol{\theta}$, considerando-se um sistema de coordenadas cilíndricas com origem em $\xi$. A integração sobre as outras duas sub-células é efetuada numericamente, da mesma maneira que a utilizada para as células que não contém o nó singular.

Calculando-se a integral (2.91.a) para cada ponto interno i e cada célula $\mathbf{j}$, gera-se a sub-matriz $\mathbf{e}^{\prime}$, semelhante àquela dada pela eq.(2.90). Esta sub-matriz 
contém a influência dos momentos iniciais da célula $\mathbf{j}$, sobre os momentos fletor e torçor no ponto $\mathbf{i}$, dada pelos pontos nodais $\mathbf{k}_{\mathbf{1}}, \mathbf{k}_{\mathbf{2}}$ e $\mathbf{k}_{\mathbf{3}}$.

Armazenando-se todos os coeficientes da sub-matriz e' de todas as células sobre todos os pontos internos, gera-se a matriz $\underset{\approx}{\mathbf{E}^{\prime}}$.

A influência do termo livre $\mathbf{g}_{\alpha \beta \gamma \theta}^{*}(\boldsymbol{\xi}) \mathbf{M}_{\gamma \theta}^{\mathbf{0}}(\boldsymbol{\xi})$, na eq.(2.40), é calculada diretamente e também armazenada na matriz $\underset{\approx}{\mathbf{E}^{\prime}}$.

Na determinação da influência dos momentos iniciais $\mathbf{M}_{\alpha \beta}^{\mathbf{0}}$ sobre os valores dos esforços cortantes em cada ponto interno, segue-se o mesmo raciocínio anterior. Calcula-se a integral dada pela eq.(2.91.b) para cada ponto interno $\mathbf{i}$ e cada célula $\mathbf{j}$,

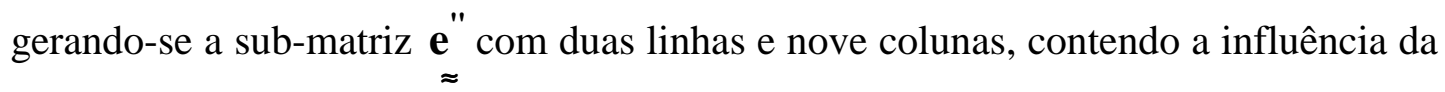
célula sobre os valores dos esforços cortantes no ponto $\mathbf{i}$.

Integrando-se a eq.(2.91.b) de todas as células para todos os pontos internos, gera-se a matriz $\mathbf{E}^{\prime \prime}$.

\subsubsection{Técnica de solução}

A discretização da equação integral de contorno apresentada no item 2.4.2. deu origem à equação matricial (2.64), a qual é reescrita a seguir:

$$
\underset{\approx \approx}{\mathbf{H}}=\underset{\approx}{\mathbf{G}} \mathbf{P}+\mathbf{B}+\underset{\approx}{\mathbf{E}} \mathbf{M}^{\mathbf{0}}
$$

Os vetores $\underset{\approx}{\mathbf{U}} \mathrm{e} \underset{\approx}{\mathbf{P}}$ contém valores conhecidos e incógnitos. Trocando-se as colunas entre as matrizes $\underset{\approx}{\mathbf{H}}$ e $\underset{\approx}{\mathbf{G}}$, de tal forma que as incógnitas do problema estejam todas num vetor $\mathbf{X}$, obtém-se:

$$
\mathbf{A X}=\mathbf{F}+\mathbf{E M}^{\mathbf{0}}
$$

Assim, o vetor $\mathbf{F}$ contém os efeitos dos deslocamentos e forças prescritas no contorno, e dos demais carregamentos atuantes no domínio, exceto o campo de 
momentos iniciais $\underset{\approx}{\mathbf{M}^{\mathbf{0}}}$. A matriz $\underset{\approx}{\mathbf{A}}$ é a matriz dos coeficientes das incógnitas, sendo em geral não simétrica e cheia.

A solução do sistema (2.92) pode ser obtida pré-multiplicando-se o primeiro e o segundo membros por $\underset{\approx}{\mathbf{A}^{-1}}$, obtendo-se:

$$
\underset{\approx}{\mathbf{X}}=\underset{\approx}{\mathbf{L}}+\underset{\mathbf{R}}{\mathbf{R}} \mathbf{M}^{\mathbf{0}}
$$

onde:

$$
\underset{\approx}{\mathbf{R}}=\underset{\mathbf{A}}{\mathbf{- 1}} \mathbf{E} ; \quad \mathbf{L}=\underset{\approx}{\mathbf{A}^{-1} \mathbf{F}}
$$

Determinadas as incógnitas do contorno, tem-se todos os valores dos vetores $\underset{\approx}{\mathbf{U}} \mathrm{e} \mathbf{P}$. A partir destes, determinam-se os valores dos deslocamentos nos pontos internos, utilizando-se o sistema dado pela eq.(2.72).

A discretização dada no item 2.4.4 para a representação dos esforços nos pontos internos conduziu às seguintes expressões:

$$
\begin{aligned}
& \mathbf{M}=\underset{\approx}{ }=\mathbf{G}^{\prime} \mathbf{P}-\mathbf{H}^{\prime} \mathbf{U}+\mathbf{B}^{\prime}+\left(\mathbf{E}^{\prime}-\mathbf{I}\right) \mathbf{M}^{\mathbf{0}} \\
& \mathbf{Q}=\mathbf{G}^{\prime} \mathbf{P}-\mathbf{H}^{\prime \prime} \mathbf{U}+\mathbf{B}^{\prime \prime}+\mathbf{E}^{\prime \prime} \mathbf{M}^{\mathbf{0}} \\
& \approx \approx \approx \approx \approx
\end{aligned}
$$

Com o mesmo procedimento utilizado na obtenção da eq.(2.93), obtém-se, para as eqs.(2.75) e (2.76):

$$
\begin{gathered}
\mathbf{M}=\mathbf{F}^{\prime}-\mathbf{A}^{\prime} \mathbf{X}+\left(\mathbf{E}^{\prime}-\mathbf{I}\right) \mathbf{M}^{\mathbf{0}} \\
\approx \approx \approx \mathbf{F}^{\prime \prime}-\mathbf{A}^{\prime \prime} \mathbf{X}+\mathbf{E}^{\prime \prime} \mathbf{M}^{\mathbf{0}} \\
\quad \approx \approx \approx \approx
\end{gathered}
$$

A eq. (2.94) dá o momento total como uma função do campo de momentos iniciais $\underset{\approx}{\mathbf{M}^{\mathbf{0}}}$, dos demais carregamentos de domínio e dos valores de contorno. Como é visto no capítulo 4, para as aplicações em problemas não-lineares, convém escrever a componente elástica do tensor momento, fazendo-se $\underset{\approx}{\mathbf{M}}=\underset{\approx}{\mathbf{M}^{\mathbf{e}}-\mathbf{M}^{\mathbf{0}}}$, onde $\mathbf{M}^{\mathbf{e}}$ é a componente elástica do momento, e $\mathbf{M}$, o momento total. Logo, podese escrever: 


$$
\underset{\approx}{\mathbf{M}^{\mathrm{e}}}=\underset{\approx}{\mathbf{F}^{\prime}-\mathbf{A}^{\prime} \mathbf{X}+\mathbf{E}^{\prime} \mathbf{M}^{\mathbf{0}}}
$$

Substituindo-se o valor de $\underset{\approx}{\mathbf{X}}$ dado pela eq.(2.93.a) na eq.(2.96), obtém-se:

ou:

$$
\begin{aligned}
& \mathbf{M}^{\mathrm{e}}=\mathbf{F}^{\prime}-\mathbf{A}^{\prime}\left(\mathbf{L}+\mathbf{R} \mathbf{M}^{\mathbf{0}}\right)+\underset{\approx}{\mathbf{E}^{\prime} \mathbf{M}^{\mathbf{0}}} \\
& \mathbf{M}^{\mathrm{e}}=\mathbf{F}^{\prime}-\mathbf{A}^{\prime} \mathbf{L}+\left(\mathbf{E}^{\prime}-\mathbf{A}^{\prime} \mathbf{R}\right) \mathbf{M}^{\mathbf{0}} \\
& \approx \approx \approx \approx \approx \approx
\end{aligned}
$$

fazendo-se:

$$
\underset{\approx}{\mathbf{N}}=\underset{\approx}{\mathbf{F}^{\prime}-\mathbf{A}^{\prime} \mathbf{L} ; \quad} \quad \mathbf{T}=\underset{\approx}{\mathbf{E}}-\mathbf{\mathbf { A } ^ { \prime }} \mathbf{R}
$$

e levando-se na eq.(2.98), obtém-se:

$$
\underset{\approx}{\mathbf{M}^{\mathrm{e}}}=\underset{\approx}{\mathbf{N}}+\underset{\mathbf{T}}{\mathbf{M}^{\mathbf{0}}}
$$

Deve-se observar que os vetores $\mathbf{L}$ e $\mathbf{N}$ representam as soluções para as incógnitas de contorno e momentos, respectivamente, quando da ausência de momentos iniciais. Na presença destes, seus efeitos são considerados pelas matrizes $\mathbf{R}$ e $\mathbf{T}$, sendo a primeira a solução para as incógnitas de contorno, e a segunda, a solução em termos de momentos nos pontos internos.

Desenvolvimento semelhante pode ser feito para os esforços cortantes, resultando em:

$$
\underset{\approx}{\mathbf{Q}} \underset{\approx}{\mathbf{N}^{\prime}}+\mathbf{T}^{\prime} \mathbf{M}^{\mathbf{0}}
$$

sendo $\underset{\approx}{\mathbf{N}^{\prime}}$ a resposta em termos de esforços cortantes na ausência do campo de momentos iniciais; na ocorrência dos mesmos, seu efeito é considerado pela matriz $\underset{\approx}{\mathbf{T}}$, onde:

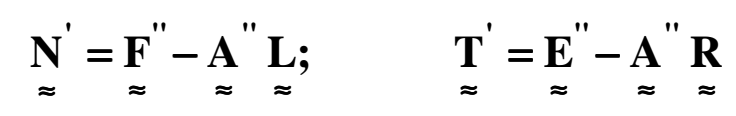




\section{CAPÍTULO III}

\section{PLACAS COM ENRIJECEDORES}

\subsection{INTRODUÇÃO}

$\mathrm{O}$ vetor $\underset{\approx}{\mathbf{B}}$ na eq.(2.64) armazena a influência de todo o carregamento externo aplicado à placa, exceto o campo de momentos iniciais. Este carregamento prescrito, que pode estar atuando em áreas internas, em pontos ou em linhas, pode também ser interpretado como reações incógnitas da interface placa-viga ou placa-pilar. Assim, para a análise de placas com enrijecedores, introduz-se uma alteração na eq.(2.64), conforme SILVA (1996), como é visto a seguir.

\subsection{APOIOS EM LINHAS}

No estudo da associação de placa-vigas, considera-se que os novos elementos estejam ligados à placa por linhas e pontos discretos. Estas linhas representam as vigas associadas à placa, com rigidez à torção e à flexão introduzindo, portanto, um enrijecimento ao sistema estrutural. Os esforços que surgem na interface das duas estruturas são interpretados como linhas de carga vertical e linhas de momentos aplicados à placa. Dessa forma, a eq.(2.64) pode ser reescrita como:

$$
\begin{aligned}
& \mathbf{H} \mathbf{U}=\mathbf{G P}+\mathbf{B}+\mathbf{E} \mathbf{M}^{\mathbf{0}}+\mathbf{S P}^{\mathbf{N}} \\
& \approx \approx \approx \approx \approx
\end{aligned}
$$

$\underset{\approx}{\text { onde }} \mathrm{p}^{\mathrm{N}}$ é o vetor que contém todos os valores nodais das linhas de carga vertical e de momentos, interpretadas como reações das vigas sobre a placa. $\underset{\approx}{S}$ é a matriz cujos coeficientes representam a influência das linhas de carga vertical e de momentos no cálculo dos deslocamentos; seus valores são obtidos a partir das eqs.(2.46) e (2.51), 
respectivamente. Como o vetor ${\underset{\sim}{N}}^{N}$ não se constitui em valores incógnitos normais à análise de placas, o mesmo deve ser transformado em deslocamentos. Para tanto, utiliza-se a seguinte expressão, válida para cada elemento de linha de carga:

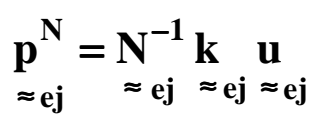

onde $\mathrm{N}_{\mathrm{j}}$ é a matriz que transforma cargas nodais distribuídas em forças nodais equivalentes $\underset{\approx \mathbf{j}}{\mathbf{F}}$, posto que $\underset{\approx}{\mathbf{F}=\mathbf{k}} \underset{\approx \mathrm{ej}}{\mathbf{u}} \underset{\approx \mathrm{ej}}{;} \underset{\approx \mathbf{j}}{\mathbf{N}}$ é função apenas das aproximações adotadas para os deslocamentos e para as cargas distribuídas. $\underset{\approx \text { ej }}{\mathbf{k}}$ é a matriz de rigidez do elemento $\mathbf{j} ; \underset{\approx \text { ej }}{\mathbf{u}}$ é o vetor dos deslocamentos nodais do elemento $\mathbf{j}$.

Resolve-se a eq.(3.2) para cada elemento de linha de carga, multiplica-se pela respectiva matriz $\underset{\approx \mathbf{j}}{\mathbf{S}}$ e armazenam-se os coeficientes de todos os deslocamentos $\underset{\approx \mathbf{e}}{\mathbf{u}}$, na matriz $\underset{\approx N K}{S}$. A eq. (3.1) pode, então, ser escrita na seguinte forma:

$$
\underset{\approx}{\mathbf{H U}}=\underset{\approx}{\mathbf{G P}}+\underset{\approx}{\mathbf{B}}+\underset{\approx \mathbf{M}^{0}}{\mathbf{E}}+\underset{\approx \mathrm{NK}}{\mathbf{S}} \mathbf{\mathrm { u }}
$$

Como o vetor dos deslocamentos $\underset{\approx \mathbf{e}}{\mathbf{u}}$ pode conter tanto deslocamentos internos como deslocamentos do contorno, a eq.(3.3) fica expressa por:

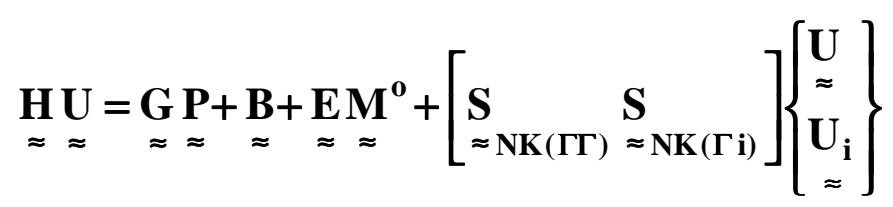

Como na eq.(3.4) introduziram-se novas incógnitas $\underset{\approx}{\mathbf{U}_{\mathbf{i}}}$, necessitam-se novas equações de deslocamentos para os pontos internos relativos ao vetor $\mathbf{U}_{\mathbf{i}}$. Agrupando-se essas novas equações à eq.(3.4), e rearranjando-se convenientemente cada termo, obtém-se:

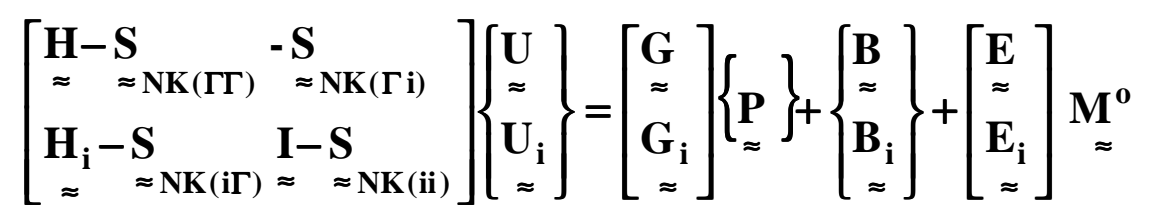


Esta equação representa a equação matricial dada pela eq.(2.64), considerando, agora, o enrijecimento produzido pelas vigas, traduzido pela aplicação das linhas de carga.

Resolvendo-se o sistema de equações dado em (3.5), obtém-se os deslocamentos nos pontos do contorno e nos pontos internos. Com isso é possível determinar o vetor $\mathbf{p}_{\approx}^{\mathbf{N}}$ para cada elemento de linha de carga. Aplicando-se essas cargas à placa, obtém-se os esforços e deslocamentos nos outros pontos internos de interesse.

\subsection{APOIOS PONTUAIS}

$\mathrm{Na}$ associação placa-pilar, quando a área de contato é pequena em relação à área da placa, pode-se admiti-la como pontual. Assim, as reações são interpretadas como cargas concentradas aplicadas no ponto de interface $\mathbf{j}$, cujo valor $\mathbf{R}_{\mathbf{j}}$ é incógnito.

Para uma placa com NAP apoios internos, a eq.(2.64) pode ser escrita da seguinte forma:

$$
\underset{\approx}{\mathbf{H}} \underset{\approx}{\mathbf{G}} \underset{\approx}{\mathbf{P}}+\underset{\approx}{\mathbf{B}}+\underset{\approx}{\mathbf{E}} \mathbf{M}^{0}+\underset{\approx}{\mathbf{S}} \mathbf{R e}
$$

Sendo $\underset{\approx}{\mathbf{R e}}$ o vetor das reações com 3 (NAP) elementos e $\underset{\approx}{\mathbf{S}}$ a matriz de dimensão 3(NNCP)x3(NAP) contendo a influência dos seguintes termos:

$$
\begin{gathered}
S_{\alpha \beta}=u_{\alpha \beta}^{*}(\xi, x) ; \quad S_{3 \alpha}=u_{3 \alpha}^{*}(\xi, x) \\
S_{i 3}=u_{i 3}^{*}(\xi, x)-\frac{v}{(1-v) \lambda^{2}} u_{i \alpha, \alpha}^{*}(\xi, x)
\end{gathered}
$$

Para cada apoio interno tem-se três reações $\mathbf{R}_{\mathbf{M}_{\boldsymbol{\alpha}}}$ e $\mathbf{R}_{\mathbf{w}}$, as quais representam os momentos nas direções $\alpha(\boldsymbol{\alpha}=1,2)$ e reação vertical, respectivamente.

Prescrevendo-se os deslocamentos nos NAP apoios internos, surgem na eq.(3.6), 3(NAP) reações incógnitas. Para que o sistema seja resolvido, são necessárias mais 3(NAP) novas equações, as quais são obtidas escrevendo-se as 
equações integrais referentes aos deslocamentos internos $\boldsymbol{\Phi}_{\mathbf{1}}, \boldsymbol{\Phi}_{\mathbf{2}}$ e w para os NAP apoios internos. Essas novas equações podem ser agrupadas e escritas como:

$$
\underset{\approx i}{U}=\underset{\approx i}{G} \underset{\approx i}{\mathbf{P}}-\underset{\approx i}{\mathbf{H}}+\underset{\approx i}{\mathbf{B}}+\underset{\approx i}{\mathbf{E}} \mathbf{M}^{\mathbf{H}}+\underset{\mathbf{S}}{\mathbf{R}}
$$

A eq.(3.8) pode ser reescrita na forma:

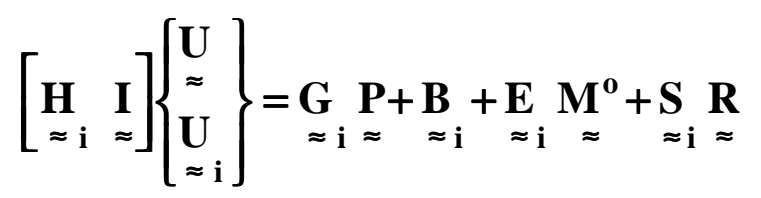

a qual pode ser agrupada à eq.(2.64) resultando:

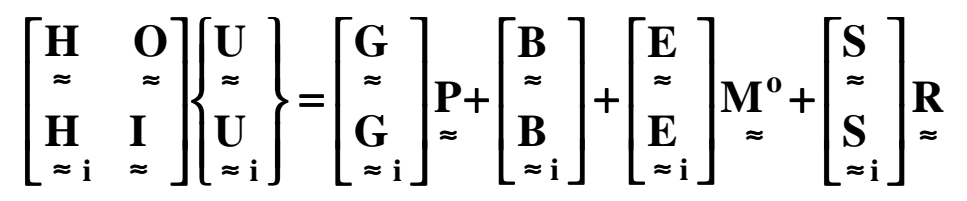

Com os valores de $\underset{\approx \mathbf{i}}{\mathbf{U}}$ prescritos, a eq.(3.10) transforma-se em:

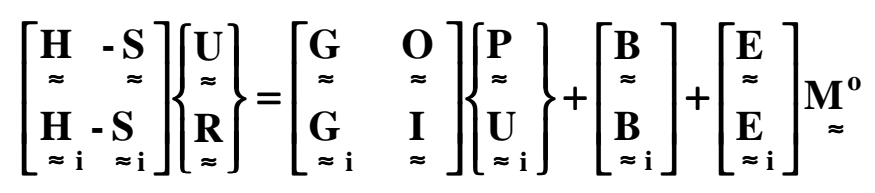

O sistema dado pela eq.(3.11) é resolvido impondo-se as condições de contorno. Assim, determinam-se as incógnitas do contorno e as reações internas $\mathbf{R}$. Com esses valores calculam-se os deslocamentos e esforços nos pontos internos de interesse.

A análise de lajes cogumelo pode ser feita considerando-se os pilares com rigidez axial infinita e rigidez à flexão nula. Assim, os deslocamentos $\mathbf{w}_{\mathbf{j}}$ e as reações $\mathbf{R}_{\mathbf{M} \boldsymbol{\alpha}}$ serão nulas, tendo-se como incógnitas apenas as reações verticais.

Escrevendo-se as equações correspondentes aos deslocamentos transversais $\mathbf{w}_{\mathbf{j}}$ para os NAP apoios internos, a eq.(3.11) fica reduzida a:

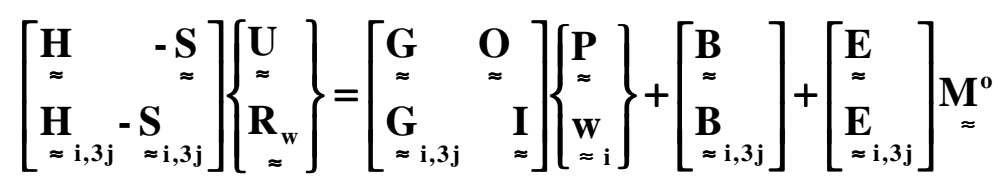




\subsection{APOIOS EM ÁREAS DISCRETAS}

Ainda quanto a associação placa-pilar, pode-se considerar o caso onde a interface é constituída por uma área. Admite-se que, após a deformação do conjunto, a área da interface permanece plana. Com isso, tem-se uma distribuição linear de tensões na interface.

Considere-se a fig. 3.1, onde estão representados uma área genérica da interface placa-pilar, o sistema de referência global $\mathbf{x}_{\mathbf{1}} \mathbf{x}_{\mathbf{2}} \mathbf{x}_{\mathbf{3}}$ e o sistema local do pilar $\overline{\mathbf{x}}_{1} \overline{\mathbf{x}}_{2} \overline{\mathbf{x}}_{3}$.

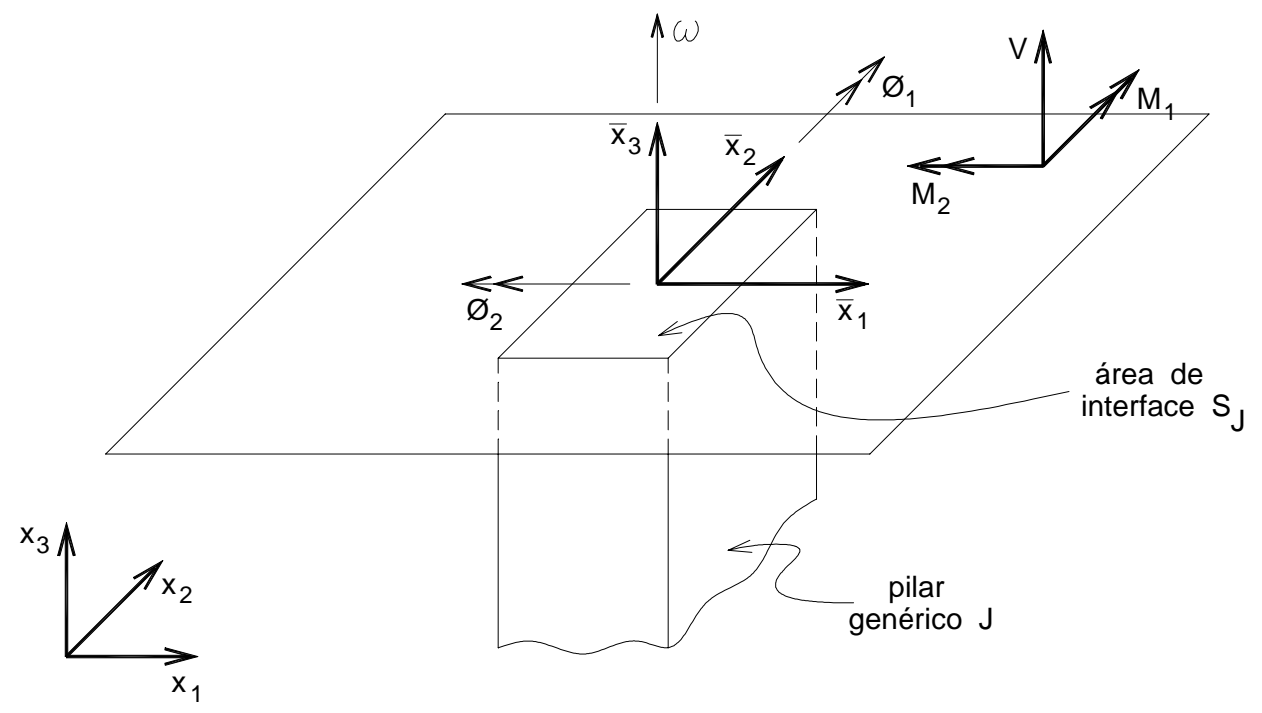

FIGURA 3.1. Associação placa - pilar A tensão num ponto $\mathbf{X}$ pertencente à área de interface é dada por:

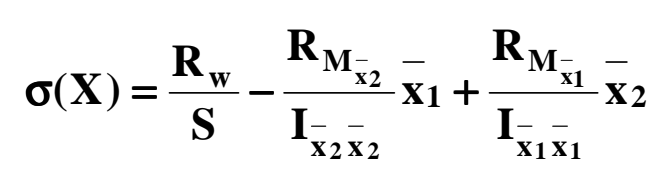

O sistema de coordenadas $\overline{\mathbf{x}}_{1} \overline{\mathbf{x}}_{2}$ representa os eixos principais de inércia da área de interface. A relação deste sistema com o sistema $\mathbf{x}_{1} \mathbf{x}_{2}$ pode ser escrita como:

$$
\begin{aligned}
& \mathbf{x}_{1}(X)=\mathbf{x}_{1}^{\mathbf{0}}+\overline{\mathbf{x}}_{1}(X) \\
& \mathbf{x}_{2}(X)=\mathbf{x}_{2}^{\mathbf{0}}+\overline{\mathbf{x}}_{2}(X)
\end{aligned}
$$


sendo $\mathbf{x}_{\mathbf{1}}^{\mathbf{0}}$ e $\mathbf{x}_{\mathbf{2}}^{\mathbf{0}}$ as coordenadas do centro da interface.

Mas, da fig.3.2., tem-se:

$$
\begin{aligned}
& \mathbf{x}_{1}(X)=x_{1}(\xi)+r \cos \theta \\
& x_{2}(X)=x_{2}(\xi)+r \operatorname{sen} \theta
\end{aligned}
$$

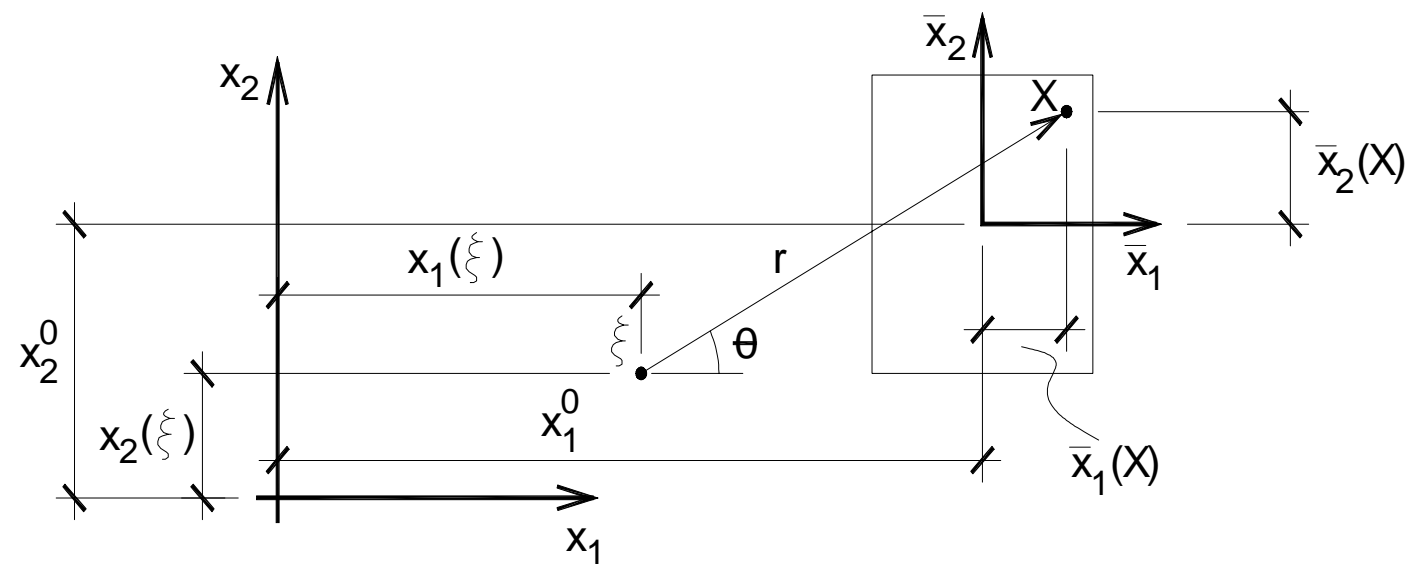

FIGURA 3.2. Sistemas de referência

Substituindo-se a eq.(3.15) na eq.(3.14) e esta na eq.(3.13) chega-se à seguinte expressão para a tensão no ponto $\mathbf{X}$ :

$$
\sigma(X)=\sigma(\xi)-\mathbf{r}\left(A_{1} \cos \theta+B_{1} \operatorname{sen} \theta\right)
$$

onde:

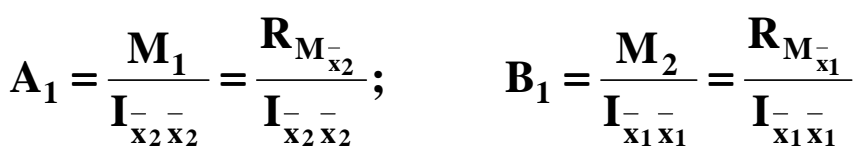

$$
\begin{aligned}
& \sigma(\xi)=\frac{\mathbf{R}_{\mathbf{w}}}{\mathrm{S}}+\mathbf{A}_{1}\left[\mathbf{x}_{1}^{\mathbf{o}}-\mathbf{x}_{1}(\xi)\right]+\mathbf{B}_{1}\left[\mathbf{x}_{2}^{\mathbf{o}}-\mathbf{x}_{2}(\xi)\right]
\end{aligned}
$$

Assim, a eq.(3.16) que representa a distribuição de tensões nos pontos pertencentes à área de interface placa-pilar, pode ser interpretada como uma carga distribuída linearmente, aplicada à placa naquela mesma área. 
Considere-se a fig.3.3, na qual são apresentadas as posições inicial e deformada para um conjunto placa-pilar.
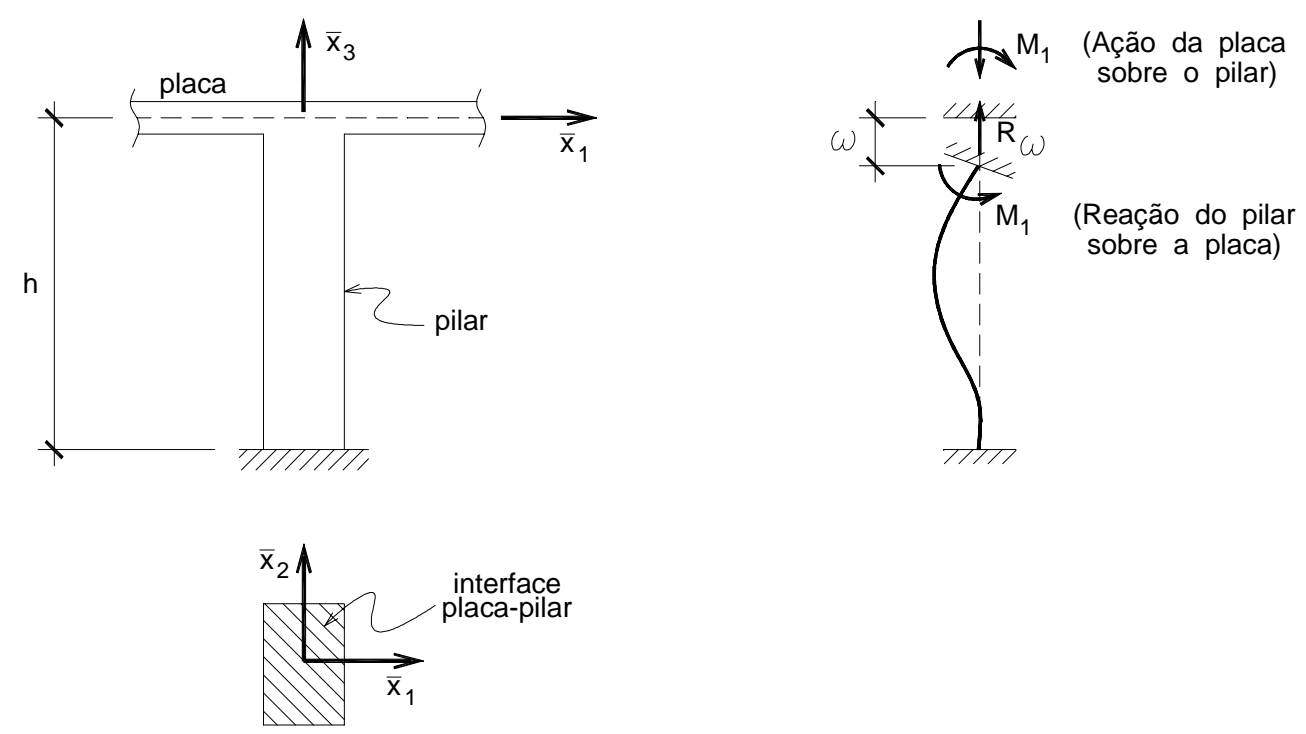

FIGURA 3.3. posições inicial e deformada do conjunto placa-pilar

Na interface, os deslocamentos $\phi_{1}, \phi_{2}$ e $\mathbf{w}$ são os mesmos, tanto para a placa quanto para o pilar.

Como deslocamentos e reações são incógnitas, deve-se relacionar tais deslocamentos com seus respectivos esforços. Como no caso em estudo os pilares formam um conjunto de barras independentes, basta considerar cada pilar isoladamente, obtendo-se a relação deslocamento-reação para cada interface. Com isso pode-se escrever:

$$
\left\{\begin{array}{l}
M_{1} \\
M_{2} \\
R_{w}
\end{array}\right\}=-\left[\begin{array}{ccc}
k_{11} & 0 & 0 \\
0 & k_{22} & 0 \\
0 & 0 & k_{33}
\end{array}\right]\left\{\begin{array}{l}
\phi_{1} \\
\phi_{2} \\
w
\end{array}\right\}
$$

onde:

$$
k_{11}=k_{p} \frac{E I_{\mathbf{x}_{2} \bar{x}_{2}}}{h} ; \quad \mathbf{k}_{22}=k_{p} \frac{E I_{\mathbf{x}_{1} \bar{x}_{1}}}{h} ; \quad k_{33}=\frac{E S}{h}
$$




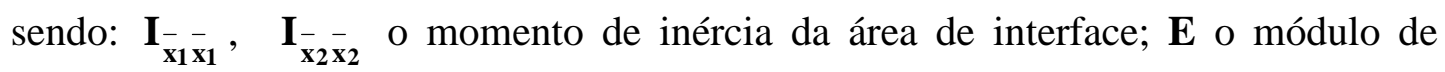
elasticidade do material do pilar; $\mathbf{S}$ é a área de interface; $\mathbf{h}$ é a altura do pilar e $\mathbf{k}_{\mathbf{p}}$ é um coeficiente que depende das condições de vinculação da base do pilar: $\mathbf{k}_{\mathbf{p}}=3$ para pilar articulado na base; $\mathbf{k}_{\mathbf{p}}=4$ para pilar engastado.

O sinal negativo na eq.(3.18) deve-se ao fato de que para deslocamentos positivos, tem-se reações negativas.

A substituição da eq.(3.18) nas eqs.(3.17) conduz a:

$$
\begin{gathered}
A_{1}=-\frac{\mathbf{k}_{\mathbf{p}} \mathbf{E}}{\mathbf{h}} \phi_{1} ; \quad \mathbf{B}_{1}=-\frac{\mathbf{k}_{\mathbf{p}} \mathbf{E}}{\mathbf{h}} \phi_{2} \\
\sigma(\xi)=-\frac{\mathbf{E}}{\mathbf{h}} \mathbf{w}+\mathbf{A}_{1}\left[\mathbf{x}_{1}^{\mathbf{o}}-\mathbf{x}_{1}(\xi)\right]+\mathbf{B}_{1}\left[\mathbf{x}_{2}^{\mathbf{o}}-\mathbf{x}_{2}(\xi)\right]
\end{gathered}
$$

Escrevendo-se a eq.(3.16) em função dos deslocamentos $\phi_{1}, \phi_{2}$ e w a partir das eqs.(3.20), obtém-se:

$$
\sigma(X)=\frac{k_{p} E}{h}\left[\left[x_{1}(\xi)-x_{1}^{0}+r \cos \theta\right] \phi_{1}+\left[x_{2}(\xi)-x_{2}^{0}+r \operatorname{sen} \theta\right] \phi_{2}\right]-\frac{E}{h} w
$$

A influência da carga distribuída $\sigma(\mathbf{X})$ na representação integral dos deslocamentos é dada pela eq.(2.45), a qual é aqui reescrita como:

$$
I_{i}=\int_{\Omega_{\mathrm{q}_{\mathrm{s}}}}\left[\mathbf{u}_{\mathrm{i} 3}^{*}(\xi, X)-\frac{v}{(1-v) \lambda^{2}} \mathbf{u}_{\mathrm{i} \alpha, \alpha}^{*}(\xi, X)\right] \sigma(X) d \Omega_{\mathrm{q}_{\mathrm{s}}}(X)
$$

Considerando a fig.3.4, a integral no domínio interno $\boldsymbol{\Omega}_{\mathbf{q}_{\mathbf{s}}}$ é transformada em uma integral de contorno sobre $\Gamma_{\mathbf{q}_{\mathrm{s}}}$.

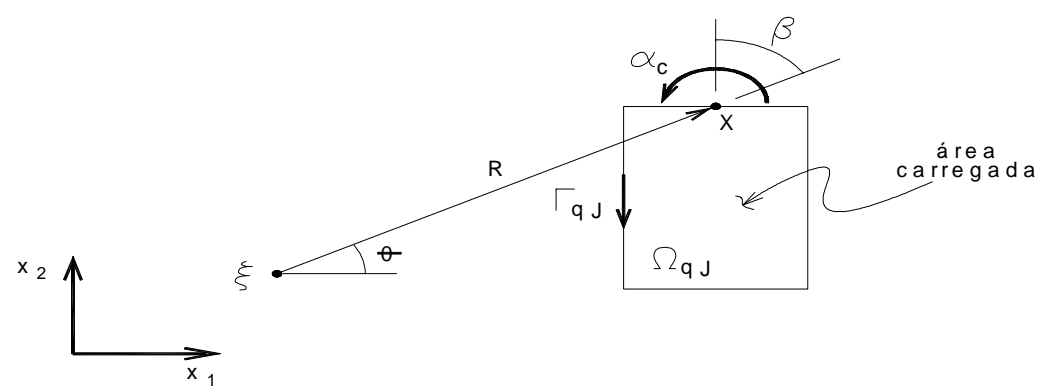

FIGURA 3.4. Domínio interno carregado com $\sigma(X)$

Esta transformação conduz às seguintes expressões: 


$$
\begin{aligned}
& I_{\alpha}=\frac{\mathbf{k}_{\mathrm{p}} \mathbf{E}}{\mathbf{h}}\left\{\left[\mathbf{x}_{1}(\xi)-\mathbf{x}_{1}^{0}\right] \mathbf{I}_{\alpha_{\mathrm{c}}}+\mathbf{I}_{\alpha_{\mathrm{r} 1}}\right\}_{1}+ \\
& +\frac{\mathbf{k}_{\mathrm{p}} \mathbf{E}}{\mathbf{h}}\left\{\left[\mathbf{x}_{2}(\xi)-\mathbf{x}_{2}^{0}\right] \mathbf{I}_{\alpha_{\mathrm{c}}}+\mathbf{I}_{\alpha_{\mathrm{r} 2}}\right\}_{2}+\frac{\mathbf{E}}{\mathbf{h}} \mathbf{I}_{\alpha_{\mathrm{c}}} \mathbf{w} \\
& \mathbf{I}_{3}=\frac{\mathbf{k}_{\mathrm{p}} \mathbf{E}}{\mathbf{h}}\left\{\left[\mathbf{x}_{1}(\xi)-\mathbf{x}_{1}^{0}\right] \mathbf{I}_{3_{\mathrm{c}}}+\mathbf{I}_{3_{\mathrm{r} 1}}\right\}_{1}+ \\
& +\frac{\mathbf{k}_{\mathrm{p}} \mathbf{E}}{\mathbf{h}}\left\{\left[\mathbf{x}_{2}(\xi)-\mathbf{x}_{2}^{0}\right] \mathbf{I}_{3_{\mathrm{c}}}+\mathbf{I}_{3_{\mathrm{r} 2}}\right\}_{2}+\frac{\mathbf{E}}{\mathbf{h}} \mathbf{I}_{3_{\mathrm{c}}} \mathbf{w}
\end{aligned}
$$

onde:

$$
\begin{aligned}
& I_{\alpha_{\mathrm{c}}}=\frac{1}{4 \pi \mathrm{D}} \int_{\Gamma_{\mathrm{q}_{\mathrm{s}}}}\left[\mathbf{R}^{2}\left(2 \ln \mathrm{z}-\frac{5}{3}\right)+\frac{12 v}{(1-v) \lambda^{2}}\right] \mathbf{r}_{, \alpha} \cos \beta d \Gamma_{\mathrm{q}_{\mathrm{s}}}(\mathrm{x}) \\
& I_{\alpha_{\mathrm{r} \beta}}=\frac{1}{32 \pi D} \int_{\Gamma_{\mathrm{q}_{\mathrm{s}}}}\left[\mathbf{R}^{3}\left(2 \ln \mathrm{z}-\frac{3}{2}\right)+\frac{8 v}{(1-v) \lambda^{2}} \mathbf{R}\right] \mathbf{r}_{, \alpha} \mathbf{r}_{, \beta} \cos \beta d \Gamma_{\mathrm{q}_{\mathrm{s}}}(\mathbf{x}) \\
& I_{3_{c}}=\frac{1}{8 \pi D(1-v) \lambda^{2}} \int_{\Gamma_{\mathrm{q}_{\mathrm{s}}}} R\left[\frac{(1-v)}{4} z^{2}\left(\ln z-\frac{5}{4}\right)+2(v-2)\left(\ln z-\frac{1}{2}\right)\right] \\
& {\left[\cos \beta d \Gamma_{q_{s}}(x)\right]} \\
& I_{3_{\mathrm{r} \alpha}}=\frac{1}{8 \pi \mathrm{D}(1-v) \lambda^{2}} \int_{\Gamma_{\mathrm{qs}}} R^{2}\left[\frac{(1-v)}{5} z^{2}\left(\ln \mathrm{z}-\frac{6}{5}\right)+\frac{4}{3}(v-2)\left(\ln \mathrm{z}-\frac{1}{3}\right)\right] \\
& {\left[\mathbf{r}_{, \alpha} \cos \beta d \Gamma_{q_{s}}(x)\right]} \\
& \mathbf{r}_{, 1}=\cos \theta ; \quad \mathbf{r}_{, 2}=\operatorname{sen} \theta \\
& \cos \beta=r_{, n}=\cos \theta \operatorname{sen} \alpha_{c}-\operatorname{sen} \theta \cos \alpha_{c}
\end{aligned}
$$

A equação matricial em termos dos deslocamentos, considerando-se os NAP apoios internos, fica:

$$
\underset{\approx}{\mathbf{H}} \underset{\approx}{\mathbf{G}} \mathbf{\mathbf { P }}+\underset{\approx}{\mathbf{B}}+\underset{\approx}{\mathbf{E}} \mathbf{M}^{\mathbf{0}}+\underset{\approx}{\mathbf{Q}} \mathbf{U}_{\mathrm{s}}
$$

sendo a matriz $\mathbf{Q}$ composta pelos coeficientes dados nas eqs.(3.23) e (3.24); o vetor $\mathbf{U}_{\mathrm{s}}$ contém os deslocamentos $\phi_{1}, \phi_{2}$ e $\mathbf{w}$ para os NAP apoios internos. Os deslocamentos na interface são os mesmos para a placa e para o pilar. Logo, pode-se escrever que $\mathbf{U}_{\mathbf{s}}=\mathbf{U}_{\mathbf{i}}$. 
Para que o sistema dado pela eq.(3.27) seja resolvido, geram-se 3(NAP) novas equações, que são aquelas correspondentes aos $\mathbf{U}_{\mathbf{i}}$ deslocamentos internos. Assim, pode-se escrever:

$$
\underset{\approx i}{U}=\underset{\approx i}{G} \underset{\approx}{P}-\underset{\approx i}{H} \underset{\approx}{U}+\underset{\approx i}{B}+\underset{\approx i}{\mathbf{E}} \underset{\approx}{M^{0}}+\underset{\approx i}{Q} \underset{\approx i}{U}
$$

Note-se que a matriz $\mathbf{Q}_{\boldsymbol{i}}$, representante da matriz $\underset{\sim}{\mathbf{Q}}$ para os pontos internos, não apresenta singularidades, uma vez que os deslocamentos nos pontos internos são calculados para o centro de gravidade da área de interface, cujas coordenadas globais são $\mathbf{x}_{\mathbf{1}}^{\mathbf{0}} \mathbf{x}_{\mathbf{2}}^{\mathbf{0}}$, e a integração no domínio $\boldsymbol{\Omega}_{\mathbf{q}_{\mathrm{s}}}$ é realizada sobre o contorno $\Gamma_{\mathbf{q}_{\mathrm{s}}}$.

A eq.(3.28) agrupada à eq.(3.27) conduz ao seguinte sistema:

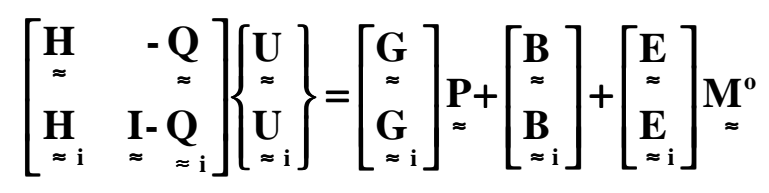

Pela imposição das condições de contorno, resolve-se o sistema dado pela eq.(3.29), obtendo-se os valores de contorno e os deslocamentos nos pontos para os NAP apoios internos. As reações nos pilares são obtidas pelo emprego da eq.(3.18). Com estes valores, obtém-se as distribuições de tensão sobre todas as áreas de interface, utilizando-se a eq.(3.16).

Para a obtenção dos deslocamentos nos outros pontos internos de interesse, utiliza-se a eq.(2.72). O vetor $\mathbf{B}_{\mathbf{i}}$ conterá a influência de todo o carregamento de domínio, incluindo as NAP regiões carregadas com as cargas $\sigma(\mathbf{X})$.

Quanto aos esforços nos pontos internos, sua obtenção se faz pela utilização das eqs. (2.75) e (2.76). Os vetores $\underset{\tilde{B}}{\mathbf{B}^{\prime}}$ e $\underset{\tilde{\mathbf{B}}}{\mathbf{B}}$ conterão a influência de todo o carregamento externo, inclusive das regiões carregadas com a carga $\boldsymbol{\sigma}(\mathbf{X})$. Neste caso, sua contribuição no cálculo dos esforços, na forma discretizada, é dada por:

$$
\begin{aligned}
& \mathbf{b}_{i}^{\prime}(\xi)=\sum_{j=1}^{\operatorname{NECC}} \frac{l_{j}}{2} \int_{-1}^{1} \sigma(X) I_{\alpha \beta}^{*}(\xi, X) d \zeta \\
& b_{i}^{\prime \prime}(\xi)=\sum_{j=1}^{\operatorname{NECC}} \frac{l_{j}}{2} \int_{-1}^{1} \sigma(X) I_{3 \beta}^{*}(\xi, X) d \zeta
\end{aligned}
$$

Onde NECC é o número de elementos de contorno da região carregada. 


\section{CAPÍTULO IV}

\section{ANÁLISE NÃO-LINEAR}

\subsection{INTRODUÇÃO}

Neste capítulo são abordados, de forma resumida, conceitos da teoria da plasticidade e da mecânica do dano, bem como suas aplicações à análise de placas. Com a aplicação dos conceitos aqui introduzidos à abordagem feita nos capítulos anteriores, é possível reproduzir, com boa aproximação, o comportamento de lajes cogumelo.

\subsection{TEORIA DA PLASTICIDADE}

A teoria da plasticidade objetiva estabelecer relações entre tensões e deformações para um determinado estado de tensão de um ponto material, que possam descrever adequadamente as deformações (elásticas e plásticas) observadas. Ocupa-se, ainda, do desenvolvimento de técnicas numéricas para implementação destas relações na análise estrutural. 


\subsubsection{CONCEITOS BÁSICOS}

\section{a) comportamento sob tração e compressão simples}

considere-se um ensaio uniaxial de tração de um corpo de prova de determinado material. Para alguns aços, as curvas tensão - deformação são como as mostradas na fig.4.1.a.

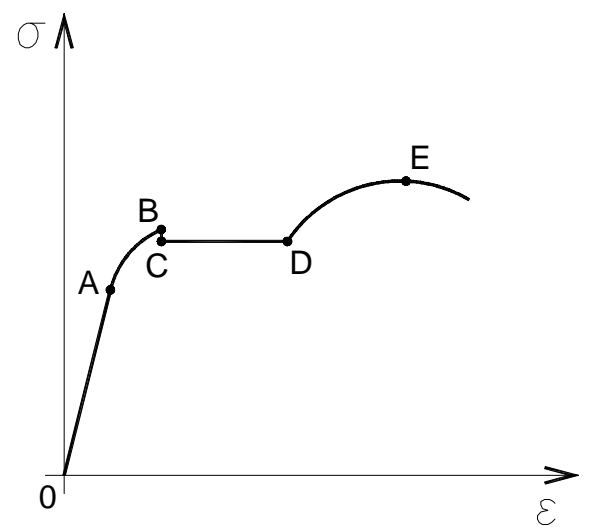

a) com patamar de escoamento

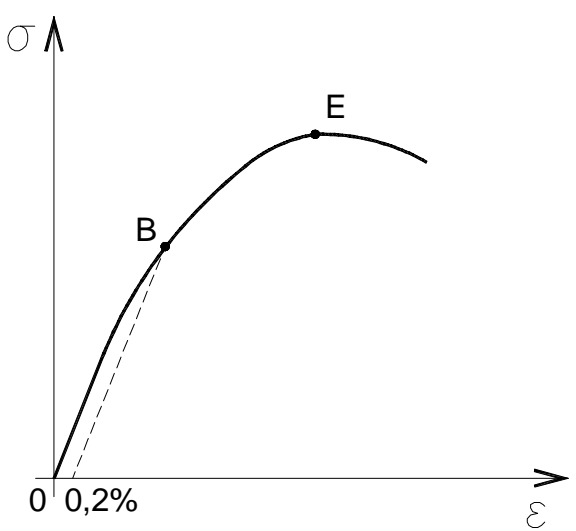

b) sem patamar de escoamento

\section{FIGURA 4.1. Diagramas tensão - deformação}

Analisando-se a fig.4.1.a., observam-se as seguintes características: um trecho reto $\mathbf{O A}$, caracterizando a região elástica linear; o ponto $\mathbf{A}$ define o limite de proporcionalidade. O trecho $\mathbf{A B}$, caracterizando a região elástica não-linear, ou seja, retirado o carregamento, o corpo de prova volta à posição inicial; o ponto $\mathbf{B}$ define o limite elástico, também chamado ponto de escoamento, uma vez que neste ponto iniciam-se as deformações plásticas irreversíveis. Observam-se, ainda, um ponto de escoamento superior $\mathbf{B}$ e um ponto de escoamento inferior C. Após o ponto $\mathbf{C}$ há uma extensão em carregamento aproximadamente constante. O comportamento na região $\mathbf{C D}$ é denominado fluxo plástico.

Para outros materiais, os pontos $\mathbf{A}, \mathbf{B}$ e o fluxo plástico $\mathbf{C D}$ não são bem definidos. A tensão de escoamento é definida por uma tensão convencional de escoamento $\sigma_{\mathrm{ys}}$, correspondente a uma deformação plástica de $0,2 \%$ (fig.4.1.b). É chamada, também, tensão inicial de escoamento. Após o ponto de escoamento, ocorrerão deformações elásticas e plásticas. A inclinação da curva decresce e, no ponto $\mathbf{E}$, ocorre a ruptura do corpo de prova. 
Há materiais em que, depois que o ponto inicial de escoamento foi alcançado, a curva tensão - deformação continua a subir, com sua inclinação tornando-se progressivamente menor, até cair a zero, quando ocorre a ruptura. As tensões de escoamento subsequentes aumentam com maiores deformações. Este efeito é denominado endurecimento. Em outras palavras, o material é capaz de resistir a maiores tensões depois de ocorridas as deformações plásticas. Outros materiais, como o concreto, num teste de compressão simples, apresentam uma região além do pico em que a inclinação da curva é negativa. Este comportamento é denominado amolecimento.

Considere-se, agora, o ensaio no qual o corpo de prova é inicialmente carregado a um valor de tensão superior à tensão inicial de escoamento, $\sigma_{\mathrm{ys}}$, e então descarregado completamente, como mostra a fig.4.2. Quando a tensão é reduzida, a

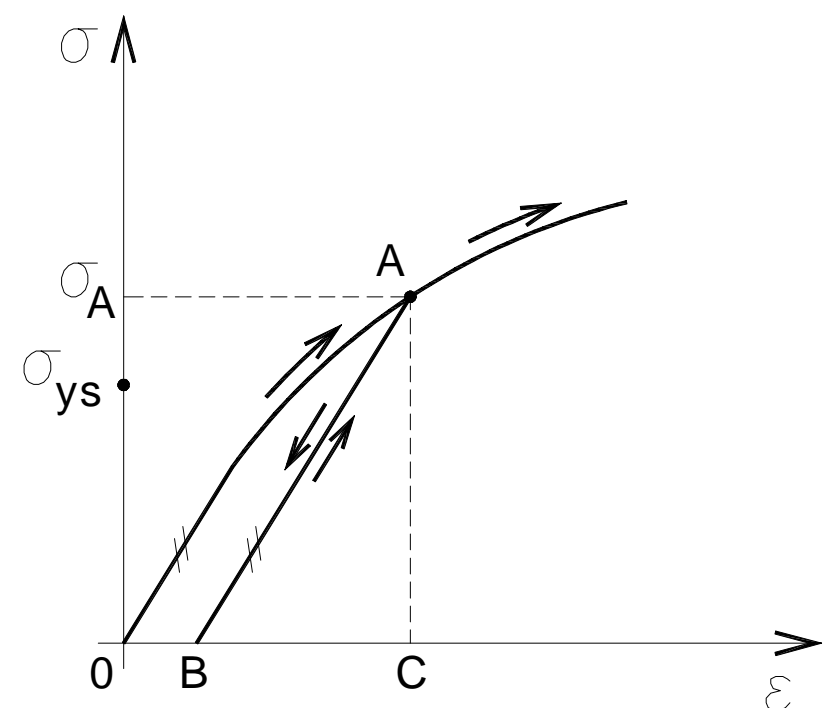

FIGURA 4.2. Ensaio uniaxial: níveis de carregamento, descarregamento e recarregamento.

deformação decresce seguindo a linha de descarregamento AB paralela ao trecho inicialmente linear da curva OA. No final do descarregamento, quando a tensão é nula, permanece uma deformação residual, ou deformação plástica OB. A deformação reversível BC é a deformação elástica. Recarregando-se o corpo de prova, a curva tensão - deformação segue a linha $\mathbf{B A}$, idêntica à linha $\mathbf{A B}$, de descarregamento. O material tem, portanto, comportamento elástico até que a tensão $\sigma_{\mathrm{A}}$ seja novamente alcançada. Esta tensão é denominada tensão de escoamento 
subsequente, acima da qual ocorrem novas deformações plásticas, seguindo a curva o mesmo trajeto do carregamento inicial.

Assim, pode-se concluir que, para o material em estudo, fixado um nível de tensão, para se determinar a deformação correspondente, é necessário conhecer a "história" do carregamento. Isto pode ser feito medindo-se a deformação plástica acumulada ou através do trabalho plástico, ou energia dissipada durante a realização de deformações irreversíveis.

\section{b) Modelos de comportamento uniaxial}

Dois modelos simplificados para o comportamento tensão - deformação do material estão representados na fig.4.3 e comentados a seguir.

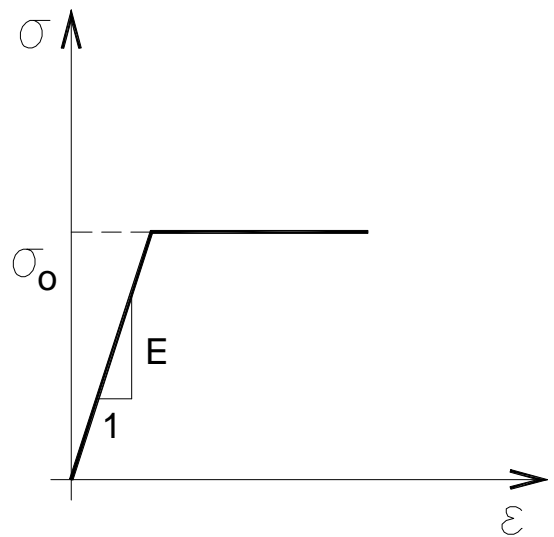

a) elastoplástico perfeito

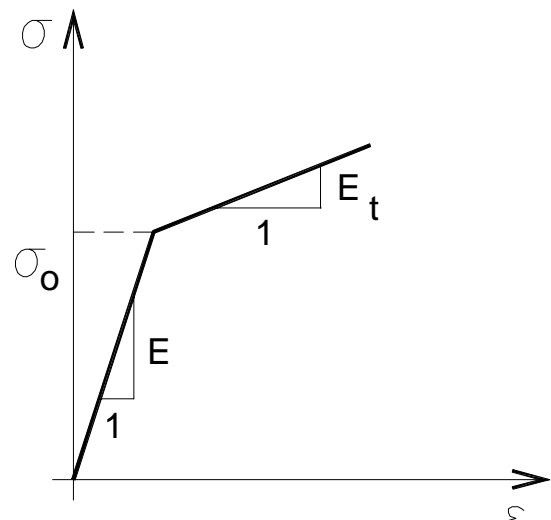

b) elastoplástico com

endurecimento

FIGURA 4.3. Modelos idealizados de comportamento

\section{b.1.) Modelo elastoplástico perfeito (fig.4.3.a)}

Neste modelo ignora-se o efeito de endurecimento e admite-se que o fluxo plástico ocorre quando a tensão alcança a tensão de escoamento $\boldsymbol{\sigma}_{\mathbf{0}}$. Até este valor, as deformações são puramente elásticas. Admite-se, ainda, a mesma resposta do material à tração e à compressão. 


\section{b.2.) Modelo elastoplástico com endurecimento (fig.4.3.b)}

Neste modelo a curva real, contínua, é aproximada por duas linhas retas, sendo a curva de transição substituída por um ponto, a ordenada do qual corresponde ao limite elástico $\sigma_{0}$. A primeira linha reta do diagrama tem uma inclinação igual ao módulo de elasticidade E. A segunda linha, representando o endurecimento, tem a inclinação $\mathbf{E}_{\mathbf{t}}<\mathbf{E}$.

\section{c) Módulo Tangente $\left(E_{t}\right)$ e módulo plástico $\left(E_{p}\right)$}

$\mathrm{Na}$ formulação elastoplástica para pequenos deslocamentos, deformação total $\boldsymbol{\varepsilon}$ pode ser expressa como a soma das parcelas elástica, $\boldsymbol{\varepsilon}^{\mathbf{e}}$, e plástica, $\boldsymbol{\varepsilon}^{\mathbf{p}}$, ou seja:

$$
\varepsilon=\varepsilon^{\mathrm{e}}+\varepsilon^{\mathrm{p}}
$$

Da mesma forma, um incremento de deformação, $\mathbf{d} \boldsymbol{\varepsilon}$, consiste de duas partes: um incremento de deformação elástica, $\mathbf{d} \boldsymbol{\varepsilon}^{\mathbf{e}}$, e um incremento de deformação plástica, $d \varepsilon^{p}$ :

$$
d \varepsilon=d \varepsilon^{e}+d \varepsilon^{p}
$$

O incremento de tensão, $\mathbf{d} \boldsymbol{\sigma}$, está relacionado ao incremento de deformação, $\mathbf{d} \varepsilon$, por:

$$
d \sigma=E_{t} d \varepsilon
$$

onde $\mathbf{E}_{\mathbf{t}}$ é o módulo tangente, que varia durante as deformações plásticas. No caso de carregamento uniaxial, $\mathbf{E}_{\mathbf{t}}$ é a inclinação corrente da curva tensão - deformação, como indica a fig.4.4.a.

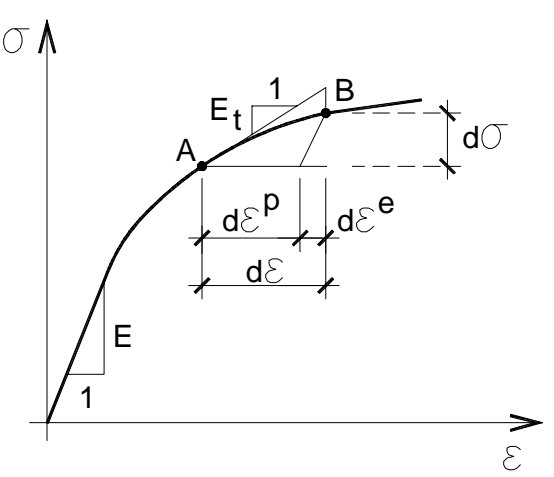

a) Módulo tangente $E_{t}$

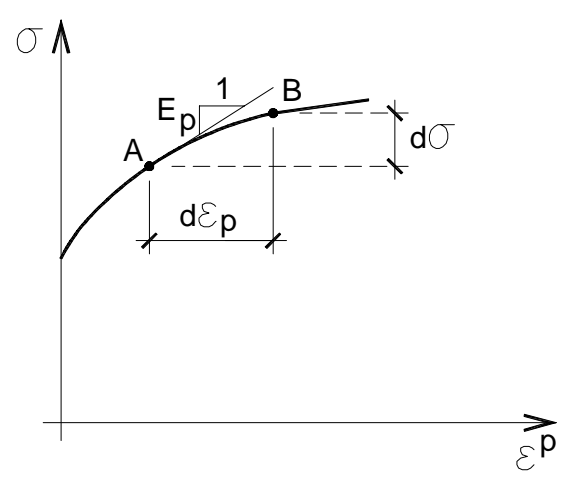

b) Módulo plástico $\mathrm{E}_{\mathrm{p}}$ 
Separando-se a deformação plástica, $\boldsymbol{\varepsilon}^{\mathbf{p}}$, da deformação total, $\boldsymbol{\varepsilon}$, o incremento de deformação plástica, $\mathbf{d} \boldsymbol{\varepsilon}^{\mathbf{p}}$, relaciona-se com o incremento de tensão, $\mathbf{d} \boldsymbol{\sigma}$, da seguinte forma:

$$
d \sigma=E_{p} d \varepsilon^{p}
$$

onde $\mathbf{E}_{\mathbf{p}}$ é o módulo plástico que, no caso de carregamento uniaxial é a inclinação da curva tensão - deformação plástica, como indica a fig.(4.4.b). Para o incremento de deformação elástica, $\mathbf{d} \boldsymbol{\varepsilon}^{\mathbf{e}}$, pode-se escrever:

$$
\mathrm{d} \sigma=\mathbf{E d} \varepsilon^{\mathrm{e}}
$$

onde $\mathbf{E}$ é o módulo elástico.

Substituindo-se $\mathbf{d} \varepsilon$ na eq.(4.3), $\mathbf{d} \varepsilon^{\mathbf{p}}$ na eq.(4.4) e $\mathbf{d} \boldsymbol{\varepsilon}^{\mathbf{e}}$ na eq.(4.5) na eq.(4.2), obtém-se a seguinte relação entre os módulos elástico, tangente e plástico:

$$
\frac{1}{E_{t}}=\frac{1}{E}+\frac{1}{E_{p}}
$$

\section{d) Regras de endurecimento}

O módulo plástico, $\mathbf{E}_{\mathbf{p}}$, pode ser escrito como uma função de um certo parâmetro de endurecimento, $\mathbf{k}$, ou seja:

$$
\mathbf{E}_{\mathbf{p}}=\mathbf{E}_{\mathbf{p}}(\mathbf{k})
$$

onde $\mathbf{k}$ pode ser tomado igual ao trabalho plástico, $\mathbf{W}_{\mathbf{p}}$, sendo:

$$
W_{p}=\int \sigma d \varepsilon^{p}
$$

ou igual a deformação plástica acumulada, $\boldsymbol{\varepsilon}^{\mathbf{p}}$, dada por:

$$
\varepsilon_{\mathrm{p}}=\int \sqrt{\mathbf{d} \varepsilon^{\mathbf{p}} \mathbf{d} \varepsilon^{\mathbf{p}}}
$$


Considere-se um elemento submetido a um teste uniaxial de carregamento reversível. A tensão de escoamento subsequente é usualmente determinada por uma das regras a seguir, como ilustra a fig.4.5.

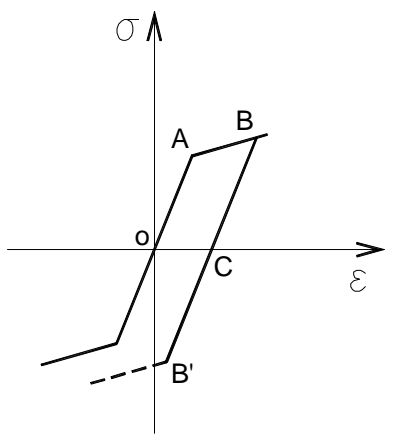

a) endurecimento isotrópico

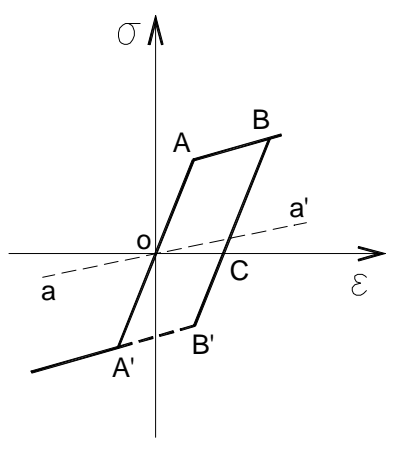

b) endurecimento cinemático

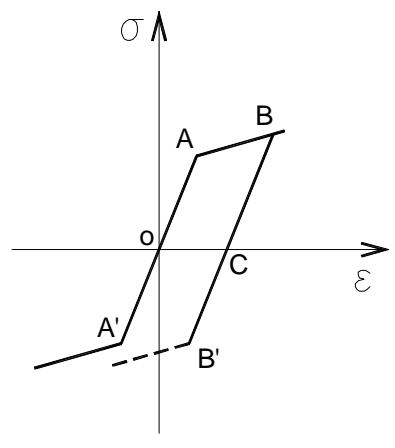

c) endurecimento independente

\section{FIGURA 4.5. Regras de endurecimento}

\section{d.1) Endurecimento isotrópico}

Esta regra assume que a tensão de escoamento de compressão na reversão do carregamento é igual à tensão de escoamento à tração antes da reversão. Como ilustra a fig.4.5.a, $\left|\overline{\mathbf{B}^{\prime} \mathbf{C}}\right|=|\overline{\mathbf{B C}}|$. Assim, a regra de endurecimento isotrópico ignora o efeito Bauschinger ${ }^{1}$. Esta regra pode ser matematicamente expressa por:

$$
|\sigma|=\mid \sigma(\mathbf{k})
$$

onde $\sigma(\mathbf{k})$ é uma função do parâmetro de endurecimento $\mathbf{k}$, e este definido como um escalar sempre positivo, tal como o trabalho plástico ou a deformação plástica acumulada.

\section{d.2) endurecimento cinemático}

Neste caso assume-se que a amplitude elástica não varia durante o endurecimento. Como se observa da fig.4.5.b, $|\overline{\mathbf{B B}}|=\left|\overline{\mathbf{A A}^{\prime}}\right|$. O centro da região elástica move-se ao longo da linha reta aa'. Esta regra é expressa matematicamente por:

\footnotetext{
${ }^{1}$ Efeito Bauschinger é o fenômeno no qual, após a primeira deformação plástica, a curva $\sigma$ - $\varepsilon$ perde a simetria e os níveis de tensão de escoamento à tração e à compressão apresentam valores distintos.
} 


$$
|\sigma-\mathbf{c}(\mathbf{k})|=\sigma_{\mathbf{o}}
$$

onde c é uma função do parâmetro de endurecimento $\mathbf{k}$.

\section{d.3) endurecimento independente}

Nesta regra considera-se que o material apresenta endurecimento independente na tração e na compressão. Da fig.4.5.c, observa-se que $\overline{\mathbf{B C}}>\overline{\mathbf{O A}}$ e $\left|\overline{\mathbf{C B}^{\prime}}\right|=\left|\overline{\mathbf{O A}^{\prime}}\right|$. O material apresenta endurecimento apenas na tração, comportandose como material virgem na condição de tensão de compressão reversa. Isto pode ser expresso como:

$$
\begin{array}{lll}
\sigma=\sigma_{t}\left(k_{t}\right) & \text { se } & \sigma>0 ; \\
\sigma=\sigma_{c}\left(k_{c}\right) & \text { se } & \sigma<0
\end{array}
$$

onde $\mathbf{k}_{\mathbf{t}}$ e $\mathbf{k}_{\mathbf{c}}$ são os parâmetros de endurecimento acumulados durante o carregamento de tração e compressão, respectivamente.

\subsubsection{Problemas multidimensionais}

A deformação total, $\varepsilon_{\mathrm{ij}}$, no caso multiaxial, pode ser expressa como a soma das componentes elástica e plástica, como no caso uniaxial, ou seja:

$$
\varepsilon_{i j}=\varepsilon_{i j}^{e}+\varepsilon_{i j}^{p}
$$

Da mesma forma, um incremento de deformação, $\mathbf{d} \boldsymbol{\varepsilon}_{\mathrm{ij}}$, pode ser decomposto nas componentes elástica e plástica:

$$
d \varepsilon_{i j}=d \varepsilon_{i j}^{e}+d \varepsilon_{i j}^{p}
$$

Para a modelagem do comportamento elastoplástico de um material, devemse especificar as seguintes relações entre tensões e deformações:

- uma relação explícita entre tensões e deformações que descreva o comportamento elástico;

- um critério de escoamento indicando o nível de tensão no qual tem início o fluxo plástico e o fim do comportamento elástico; e 
- uma relação tensão - deformação para o comportamento pós escoamento em que se obtém a componente de deformação plástica.

$\mathrm{Na}$ formulação que se segue, onde pertinente, subentende-se regra de endurecimento isotrópico.

$\mathrm{Na}$ fase elástica de comportamento, tem-se a seguinte relação entre as componentes de tensão e deformação:

$$
\sigma_{\mathrm{ij}}=\mathrm{C}_{\mathrm{ijkl}} \varepsilon_{\mathrm{kl}}^{\mathrm{e}}
$$

onde $\mathbf{C}_{\mathbf{i j k}}$ é o tensor de constantes elásticas dado pela expressão:

$$
\mathrm{C}_{\mathrm{ijkl}}=\lambda \delta_{\mathrm{ij}} \delta_{\mathrm{kl}}+\mu \delta_{\mathrm{ik}} \delta_{\mathrm{jl}}+\mu \delta_{\mathrm{il}} \delta_{\mathrm{jk}}
$$

sendo $\boldsymbol{\lambda}$ e $\boldsymbol{\mu}$ as constantes de Lamé.

Um critério de escoamento que determina a transição entre o regime elástico e plástico, para estados multiaxiais de tensão, pode ser escrito na forma:

$$
\mathbf{F}\left(\sigma_{\mathrm{ij}}, \mathbf{k}\right)=\mathbf{f}\left(\sigma_{\mathrm{ij}}\right)-\mathbf{Y}(\mathbf{k})=\mathbf{0}
$$

sendo $\mathbf{f}\left(\sigma_{\mathrm{ij}}\right)$ função do estado de tensão atual $\sigma_{\mathrm{ij}}$, podendo ser interpretada como uma tensão equivalente uniaxial $\overline{\boldsymbol{\sigma}}$. A função $\mathbf{Y}(\mathbf{k})$, que depende do parâmetro $\mathbf{k}$, associado à regra de endurecimento do material, é a tensão convencional de escoamento, obtida a partir de ensaios uniaxiais, ou a nova tensão de escoamento, caso já se tenha iniciado o processo de endurecimento do material.

De modo parecido ao que ocorre no caso uniaxial, deve-se observar a existência de uma superfície inicial de escoamento, que, no caso, é função apenas do estado de tensão. Assim, da eq.(4.17) pode-se escrever:

$$
\mathbf{F}\left(\sigma_{\mathrm{ij}}\right)=\mathbf{0}
$$

Por esta representação, acréscimos do estado de tensão que conduzam a valores negativos de $\mathbf{F}$ indicam situação de descarga ou entrada no regime elástico; acréscimos de tensão nos quais $\mathbf{F = 0}$, indicam uma situação limite ou de carregamento neutro; por outro lado, acréscimos de tensão que conduzam a valores positivos de $\mathbf{F}$ são ditos inacessíveis, e indicam o aparecimento de deformações permanentes. Nesta situação, a superfície inicial de escoamento deve evoluir no espaço das tensões de forma que o ponto que representa o novo estado de tensão resulte, ainda, sobre a superfície. Logo, um incremento no estado de tensão que produza deformação plástica denomina-se carregamento; incremento que tende a 
levar o ponto representativo do estado de tensão para o interior da superfície é chamado descarregamento. Este incremento é sempre elástico. Portanto, a superfície de escoamento em determinado estágio só sofre alteração, no espaço de tensões, quando há um processo de carregamento.

Assim, para materiais que apresentam endurecimento, após o escoamento inicial, ocorre aumento no limite elástico; o nível de tensão para o qual ocorre uma deformação plástica adicional depende do grau da deformação atual. Como conseqüência, a superfície de escoamento varia a cada estágio de deformações plásticas, gerando superfícies subsequentes. O processo de evolução das superfícies é, ainda, definido pela regra de endurecimento, que indica como a função $\mathbf{F}\left(\sigma_{i \mathbf{i j}}, \mathbf{k}\right)$ se altera.

Como no caso uniaxial, o parâmetro $\mathbf{k}$ pode ser definido de duas maneiras: a primeira, conhecida como work hardening, define $\mathbf{k}$ como uma função do trabalho plástico, $\mathbf{W}_{\mathbf{p}}$, acumulado durante as deformações, ou seja:

$$
k=W_{p}=\int \sigma_{i j} d \varepsilon_{i j}^{p}
$$

sendo $\mathbf{d} \varepsilon_{\mathbf{i j}}^{\mathbf{p}}$ a parcela plástica das deformações ocorridas durante um incremento de deformação. Na segunda hipótese, o parâmetro $\mathbf{k}$ é relacionado à uma medida de deformação total, denominada deformação plástica efetiva ou equivalente, definida por:

$$
d \bar{\varepsilon}^{-p}=\sqrt{\frac{2}{3} d \varepsilon_{i j} d \varepsilon_{i j}}
$$

Assim, o parâmetro de endurecimento $\mathbf{k}$ fica definido como:

$$
\begin{gathered}
\mathbf{k}=\overline{\boldsymbol{\varepsilon}} \\
\text { onde: } \quad \bar{\varepsilon}^{-\mathbf{p}}=\int \mathbf{d} \bar{\varepsilon}^{-\mathbf{p}}
\end{gathered}
$$

Esta hipótese é denominada strain hardening.

O caso multiaxial pode ser associado ao estado de tensão uniaxial, através da tensão equivalente, $\overline{\boldsymbol{\sigma}}$, e da deformação equivalente, $\overline{\boldsymbol{\varepsilon}}$, que reproduzem a tensão $\boldsymbol{\sigma}$ e a deformação plástica $\varepsilon^{\mathbf{p}}$, do caso uniaxial. Assim, a relação incremental pode ser expressa por: 


$$
\mathbf{d} \overline{\boldsymbol{\sigma}}=\mathbf{H}^{\prime} \mathbf{d} \bar{\varepsilon}^{-\mathbf{p}}
$$

sendo H' o parâmetro de endurecimento definido como:

$$
H^{\prime}=\frac{E_{t}}{1-\frac{E_{t}}{E}}
$$

sendo $\mathbf{E}$ o módulo de elasticidade e $\mathbf{E}_{\mathbf{t}}$ o módulo tangente.

Considere-se a definição da função de escoamento dada na eq.(4.17). Observa-se que, se $\mathbf{f}<\mathbf{Y}(\mathbf{k})$, tem-se um comportamento elástico do material; se $\mathbf{f}=\mathbf{Y}(\mathbf{k})$, inicia-se o escoamento e uma mudança incremental na função de escoamento, devida a um incremento de tensão, é dada por:

$$
\mathbf{d f}=\frac{\partial \mathbf{f}}{\partial \sigma_{\mathrm{ij}}} \mathbf{d} \sigma_{\mathrm{ij}}
$$

Assim, têm-se três situações possíveis:

a) df < 0: ocorre descarregamento (comportamento elástico) e o ponto representativo do estado de tensões retorna para o interior da superfície de escoamento;

b) $\mathrm{df}=0$ : ocorre carregamento neutro (materiais de comportamento elastoplástico perfeito), ficando o ponto sobre a superfície inicial, que é fixa;

c) df > 0: ocorre carregamento plástico (materiais de comportamento elastoplástico com endurecimento) e o ponto permanece na superfície subsequente.

As relações constitutivas para a fase pós escoamento podem ser obtidas, utilizando-se uma hipótese adicional da teoria da plasticidade segundo a qual o incremento de deformação plástica é proporcional ao gradiente de uma grandeza denominada potencial plástico, $\mathbf{Q}$, da seguinte forma:

$$
\mathbf{d} \varepsilon_{\mathrm{ij}}^{\mathrm{p}}=\mathbf{d} \lambda \frac{\partial \mathrm{Q}}{\partial \sigma_{\mathrm{ij}}}
$$

sendo $\mathbf{d} \boldsymbol{\lambda}$ uma constante denominada multiplicador plástico.

A condição dada pela eq.(4.26) é denominada regra de fluxo, uma vez que indica o fluxo plástico depois do escoamento. Quando se considera o potencial plástico, $\mathbf{Q}$, coincidente com a função de escoamento $\mathbf{F}\left(\sigma_{\mathbf{i j}}, \mathbf{k}\right)$, diz-se que a lei de fluxo é associativa. Assim, a eq.(4.26) pode ser escrita da seguinte forma: 


$$
\mathbf{d} \varepsilon_{\mathrm{ij}}^{\mathrm{p}}=\mathbf{d} \lambda \frac{\partial \mathbf{F}\left(\sigma_{\mathrm{ij}}, \mathbf{k}\right)}{\partial \sigma_{\mathrm{ij}}}=\mathbf{d} \lambda \frac{\partial \mathbf{f}\left(\sigma_{\mathrm{ij}}\right)}{\partial \sigma_{\mathrm{ij}}}
$$

uma vez que $\mathbf{Y}(\mathbf{k})$ não depende do estado de tensão.

A eq.(4.27) é denominada condição de normalidade, dado que o vetor $\partial \mathbf{F} / \partial \sigma_{\mathrm{ij}}$ é normal à superfície de escoamento no ponto representativo do estado de tensão considerado.

É possível demonstrar que tanto a superfície inicial de escoamento quanto às superfícies subsequentes, devem ser convexas. Isto se deve à lei associativa de escoamento e à condição de irreversibilidade das deformações plásticas, que implica na realização de trabalho plástico positivo durante um ciclo completo de tensão, onde as variações de tensão e de deformação devem ser de mesmo sinal.

A condição de normalidade do fluxo plástico e convexidade da superfície de escoamento são condizentes aos materiais de comportamento elastoplástico perfeito ou com endurecimento, o que garante a unicidade da solução do problema.

Substituindo-se o incremento de deformação elástica da eq.(4.14) na eq.(4.15), obtém-se:

$$
d \sigma_{i j}=C_{i j k l}\left(d \varepsilon_{k l}-d \varepsilon_{k l}^{p}\right)
$$

Tendo em vistas o incremento de deformação plástica dado na eq.(4.27), pode-se escrever:

$$
d \sigma_{i j}=C_{i j k l}\left(d \varepsilon_{k l}-a_{k l} d \lambda\right)
$$

onde:

$$
\mathbf{a}_{\mathrm{kl}}=\frac{\partial \mathbf{F}\left(\sigma_{\mathrm{kl}}, \mathbf{k}\right)}{\partial \sigma_{\mathrm{kl}}}=\frac{\partial \mathbf{f}\left(\sigma_{\mathrm{kl}}\right)}{\partial \sigma_{\mathrm{kl}}}
$$

Durante a ocorrência de deformações plásticas, $\mathbf{d F}=\mathbf{0}$, ou seja:

$$
\mathbf{d F}=\frac{\partial \mathbf{F}}{\partial \sigma_{i j}} \mathbf{d} \sigma_{i j}+\frac{\partial F}{\partial k} \mathbf{d k}=\mathbf{0}
$$

ou:

$$
\mathbf{a}_{\mathrm{ij}} \mathbf{d} \sigma_{\mathrm{ij}}-\frac{\partial \mathbf{Y}}{\partial \mathbf{k}} \mathbf{d k}=\mathbf{0}
$$


Da eq.(4.19) pode-se escrever:

$$
\mathbf{d k}=\sigma_{\mathrm{ij}} \mathbf{d} \varepsilon_{\mathrm{ij}}^{\mathrm{p}}=\sigma_{\mathrm{ij}} \mathrm{d} \lambda \frac{\partial \mathbf{f}\left(\sigma_{\mathrm{ij}}\right)}{\partial \sigma_{\mathrm{ij}}}
$$

Substituindo-se a eq.(4.33) na eq.(4.32), chega-se a:

$$
\mathbf{a}_{\mathrm{ij}} \mathrm{d} \sigma_{\mathrm{ij}}-\frac{\partial \mathrm{Y}(\mathrm{k})}{\partial \mathrm{k}} \sigma_{\mathrm{ij}} \mathbf{a}_{\mathrm{ij}} \mathrm{d} \lambda=\mathbf{0}
$$

O segundo termo desta equação pode ser reescrito na forma:

$$
\frac{\partial Y(k)}{\partial k} \sigma_{i j} a_{i j} d \lambda=\frac{\partial Y(k)}{\partial k} \sigma_{i j} \frac{d f\left(\sigma_{i j}\right)}{d \sigma_{i j}} d \lambda=0
$$

Sendo $\mathbf{f}$ uma função homogênea de grau unitário, aplicando-se o Teorema de Euler para funções homogêneas na eq.(4.35), obtém-se:

$$
\frac{\partial \mathbf{Y}(\mathbf{k})}{\partial \mathbf{k}} \boldsymbol{\sigma}_{\mathrm{ij}} \mathbf{a}_{\mathrm{ij}} \mathbf{d} \lambda=\frac{\partial \mathbf{Y}(\mathbf{k})}{\partial \mathbf{k}} \mathbf{f}\left(\boldsymbol{\sigma}_{\mathrm{ij}}\right) \mathbf{d} \lambda
$$

Através da definição do parâmetro de endurecimento em função do trabalho plástico, definido na eq.(4.19) e considerando-se $\mathbf{f}\left(\sigma_{\mathrm{ij}}\right)$ como uma tensão equivalente $\overline{\boldsymbol{\sigma}}$ para o caso uniaxial, pode-se escrever:

$$
\frac{\partial \mathbf{Y}(\mathbf{k})}{\partial \mathbf{k}} \mathbf{f}\left(\sigma_{\mathrm{ij}}\right) \mathbf{d} \lambda=\mathbf{H}^{\prime} \mathbf{d} \lambda
$$

Substituindo-se a eq.(4.37) na eq.(4.36) e esta na eq.(4.34), tem-se:

$$
\mathbf{a}_{\mathrm{ij}} \mathbf{d} \sigma_{\mathrm{ij}}-H^{\prime} \mathbf{d} \lambda=\mathbf{0}
$$

$\mathrm{O}$ valor de $\mathbf{d} \boldsymbol{\lambda}$ pode ser determinado em função do incremento total de deformação, utilizando-se as eqs.(4.29) e (4.38), obtendo-se:

$$
d \lambda=\frac{a_{i j} C_{i j k l}}{\left(a_{i j} d_{i j}+H^{\prime}\right)} d \varepsilon_{k l}
$$

onde: 


$$
\mathbf{d}_{\mathrm{ij}}=\mathbf{C}_{\mathrm{ijkl}} \mathbf{a}_{\mathrm{kl}}
$$

Substituindo-se a eq.(4.39) na eq.(4.29), obtém-se, finalmente:

$$
d \sigma_{i j}=\left(C_{i j k l}-\frac{d_{i j} a_{m n} C_{m n k l}}{d_{m n} a_{m n}+H^{\prime}}\right) d \varepsilon_{k l}
$$

ou:

$$
d \sigma_{\mathrm{ij}}=C_{\mathrm{ijkl}}^{\mathrm{ep}} \mathrm{d} \varepsilon_{\mathrm{kl}}
$$

onde:

$$
C_{i j k l}^{e p}=C_{i j k l}-\frac{d_{i j} a_{m n} C_{m n k l}}{d_{m n} a_{m n}+H^{\prime}}
$$

O incremento de tensão dado na eq.(4.41) pode ser expresso através das componentes elástica e plástica como:

$$
d \sigma_{i j}=d \sigma_{i j}^{e}-d \sigma_{i j}^{p}
$$

sendo:

$$
d \sigma_{i j}^{e}=C_{i j k l} d \varepsilon_{k l}
$$

a componente plástica pode ser escrita por:

$$
d \sigma_{i j}^{p}=\frac{d_{i j} a_{m n}}{d_{m n} a_{m n}+H^{\prime}} d \sigma_{m n}^{e}
$$

em que $\mathbf{d} \boldsymbol{\sigma}_{\mathbf{m n}}^{\mathbf{e}}$ representa o incremento de tensão, devido ao incremento total de deformação, supondo o comportamento elástico. Sua determinação é dada pela eq.(4.45).

Pode-se escrever as relações constitutivas na forma matricial. Assim, a eq.(4.41), que relaciona os incrementos de tensão e deformação após o escoamento, fica expressa por:

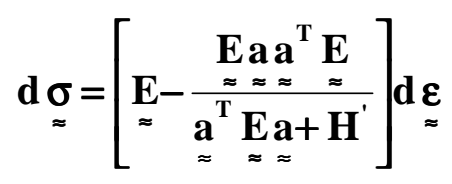

sendo $\underset{\approx}{\mathbf{E}}$ a matriz de constantes elásticas e $\underset{\approx}{\mathbf{a}}$, o vetor de fluxo, dado por: 


$$
\underset{\approx}{\mathbf{a}}=\frac{\partial \mathbf{F}}{\partial \sigma}
$$

e:

$$
\underset{\approx}{\sigma^{\mathrm{T}}}=\left\{\begin{array}{llllll}
\sigma_{11} & \sigma_{22} & \sigma_{33} & \sigma_{23} & \sigma_{31} & \sigma_{12}
\end{array}\right\}
$$

Assim, para a determinação da matriz elastoplástica indicada na eq.(4.47), é suficiente o conhecimento do vetor de fluxo para cada função de escoamento.

NAYAK \& ZIENKIEWICZ (1972), expressam o vetor de fluxo $\underset{\approx}{\mathbf{a}}$ para todos os critérios de escoamento na seguinte forma geral:

$$
\underset{\approx}{\mathbf{a}}=\mathrm{C}_{1} \underset{\approx 1}{\mathbf{a}}+\mathrm{C}_{2} \underset{\approx 2}{\mathbf{a}}+\mathrm{C}_{3} \underset{\approx 3}{\mathbf{a}}
$$

onde:

$$
\underset{\approx 1}{\mathbf{a}^{\mathrm{T}}}=\frac{\partial \mathrm{J}_{1}}{\partial \underset{\approx}{\sigma} ;} \quad \underset{\approx 2}{\mathbf{a}^{\mathrm{T}}}=\frac{\partial\left(\mathbf{j}_{2}\right)^{1 / 2}}{\partial \underset{\approx}{\sigma}} ; \quad \underset{\approx 3}{\mathbf{a}^{\mathrm{T}}}=\frac{\partial \mathbf{J}_{3}}{\partial \sigma}
$$

Dessa forma, apenas as constantes $\mathbf{C}_{\mathbf{1}}, \mathbf{C}_{\mathbf{2}}$ e $\mathbf{C}_{\mathbf{3}}$ dependem da função de escoamento. Para o critério de Tresca, tem-se:

$$
C_{1}=0 ; \quad C_{2}=2 \cos \theta(1+\operatorname{tg} \theta \operatorname{tg} 3 \theta) ; \quad C_{3}=\frac{\sqrt{3}}{J_{2}} \frac{\operatorname{sen} \theta}{\cos 3 \theta}
$$

Para o critério de von Mises estas constantes valem:

$$
\mathrm{C}_{1}=0 ; \quad \mathrm{C}_{2}=\sqrt{3} ; \quad \mathbf{C}_{3}=\mathbf{0}
$$




\subsection{MECÂNICA DO DANO CONTÍNUO}

\subsubsection{Introdução}

Apresenta-se aqui, de forma sucinta, aspectos básicos da mecânica do dano contínuo aplicada ao concreto, bem como o modelo de dano de Mazars, utilizado no presente trabalho. Maiores detalhes podem ser encontrados nos trabalhos de MAZARS (1984), PEREGO (1990), ÁLVARES (1993), e BUSSAMRA (1993).

O dano pode ser definido como o processo de aparecimento e crescimento de microfissuras levando a um deterioramento do material e conseqüente alteração de suas características mecânicas.

O objetivo final da mecânica do dano contínuo pode ser apresentado como sendo a formulação de leis constitutivas que permitam prever, com suficiente precisão, a resposta não linear de estruturas em correspondência a ações externas.

Vários modelos constitutivos baseados na mecânica do dano, dentre eles o modelo de Mazars, têm como fundamento a termodinâmica dos processos irreversíveis.

Posto que na termodinâmica clássica os sistemas são estudados através de equações de estado onde as hipóteses são a reversibilidade dos processos e que a danificação de materiais sólidos é um processo claramente irreversível, faz-se necessária a generalização dos conceitos da termodinâmica para que seja, assim, incluída a irreversibilidade.

As teorias termodinâmicas estudam processos que podem ser definidos como quaisquer alterações em um sistema. Segundo Pimenta apud Bussamra ${ }^{\text {a }}$ termodinâmica dos sólidos deformáveis trata de processos irreversíveis em sistemas sólidos.

Segundo Bussamra (1993), uma alteração em um sistema pode ser identificada a partir de um estado termodinâmico em cada instante bem definido. Para tanto, definem-se as variáveis termodinâmicas e os seguintes postulados:

\footnotetext{
${ }^{2}$ PIMENTA, P.M. Análise de sólidos elastoplásticos, São Paulo, 1987. Tese (Livre Docência). Escola Politécnica da Universidade de São Paulo.

${ }^{3}$ BUSSAMRA, F.L.S. Equações constitutivas do concreto baseadas na mecânica do dano contínuo. São Paulo, 1993. Dissertação (Mestrado). Escola Politécnica da Universidade de São Paulo.
} 
$1^{\mathrm{o}}$. qualquer parte do sólido pode ser considerada um sistema;

$2^{\circ}$. todo sistema possui uma energia interna $\mathbf{U}$.

Sendo a energia interna $\mathbf{U}$ uma propriedade extensiva de um sistema, ou seja, que depende de sua massa. Isto posto, denomina-se u como a energia interna por unidade de massa.

Seja um meio contínuo genérico de área de superfície $\Gamma$ e volume $\Omega$, sujeito a uma força de superfície $\underset{\approx}{\mathbf{P}}$ e uma força de volume $\underset{\approx}{\mathbf{b}}$, por unidade de superfície e de volume, respectivamente.

A primeira lei da termodinâmica, que expressa o princípio da conservação da energia, pode ser escrita na seguinte forma integral (PEREGO, 1990):

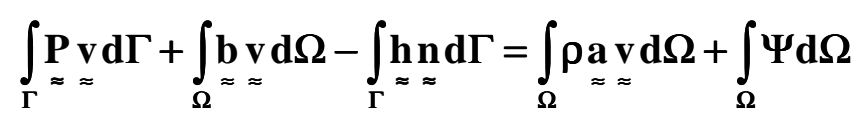

onde:

$\underset{\approx}{\mathbf{v}}=$ vetor velocidade; $\underset{\approx}{\mathbf{a}}=$ vetor aceleração; $\rho=$ densidade volumétrica; $\underset{\approx}{\mathbf{h}}=$ fluxo de calor por unidade de área; $\Psi=$ energia interna por unidade de volume.

Segundo Pimenta (1987) apud Bussamra (1993), um sistema é reversível se a sua energia interna específica $\mathbf{u}$ e o tensor das tensões $\boldsymbol{\sigma}$ são funções de estado das variáveis de estado $\boldsymbol{\varepsilon}$ (tensor das deformações) e de $\mathbf{S}$ (entropia específica), ou seja:

$$
\mathbf{u}=\mathbf{u}(\underset{\approx}{\varepsilon}, \mathbf{S}) ; \quad \underset{\approx}{\sigma} \underset{\approx}{\sigma(\varepsilon, S)}
$$

Se assim não for, o sistema é dito irreversível, o que requer a introdução de novas variáveis para definir tal estado termodinâmico.

Seja o axioma básico da termodinâmica dos processos irreversíveis: "é sempre possível encontrar um conjunto de variáveis de estado que defina completamente o estado termodinâmico de um sistema irreversível." Assim, pode-se resolver o problema introduzindo-se novas variáveis termodinâmicas, denominadas "variáveis internas" e designadas por $\pi_{\mathrm{k}}$ ou pelo tensor $\prod_{\boldsymbol{\tau}}$. Logo, tem-se para as eqs. (4.58):

$$
\mathbf{u}=\mathbf{u}(\underset{\approx}{\varepsilon}, \mathbf{S}, \underset{\approx}{\pi}) ; \quad \underset{\approx}{\sigma}=\underset{\approx}{\sigma} \underset{\approx}{\varepsilon}, \mathbf{S}, \underset{\approx}{\pi})
$$

Considere-se, ainda, a inequação: 


$$
\dot{S}+\frac{1}{\rho} \operatorname{div}\left(\frac{h}{\frac{\hat{T}}{T}}\right) \geq 0
$$

conhecida como "desigualdade de Clausius - Duhen". T é a temperatura absoluta. Esta desigualdade é válida para processos irreversíveis e a igualdade só é válida para processos reversíveis.

A conservação da quantidade de movimento, admitida a hipótese de pequenos deslocamentos, pode ser expressa por:

$$
\int_{\Gamma} \underset{\approx}{\mathbf{v}} \mathrm{d} \Gamma+\int_{\Omega} \mathbf{b v} \mathbf{v} \mathrm{d} \Omega=\int_{\Omega} \underset{\approx}{\sigma \varepsilon} \mathrm{d} \Omega+\int_{\Omega} \underset{\approx}{\operatorname{av}} \boldsymbol{v} \mathrm{d} \Omega
$$

A partir da aplicação do teorema divergente na eq.(4.57) e utilizando-se a eq.(4.61) é possível demonstrar que:

$$
\left(\underset{\approx}{\sigma-\frac{\partial \Psi}{\partial \varepsilon}}\right) \underset{\approx}{\varepsilon}+\left(\rho \mathrm{T}-\frac{\partial \Psi}{\partial S}\right) \dot{S}-\frac{\partial \Psi}{\partial \prod_{\approx}} \underset{\approx}{\dot{\Pi}}-\left(\frac{1}{T}\right) \underset{\approx}{h} \operatorname{divT} \geq 0
$$

Dado que $\dot{\mathbf{S}}$ e $\underset{\approx}{\dot{\varepsilon}}$ podem ser quaisquer, visto que são independentes, a validade da eq.(4.62) é garantida sob as seguintes condições:

$$
\underset{\approx}{\sigma}=\frac{\partial \Psi}{\partial \varepsilon} ; \quad \rho T=\frac{\partial \Psi}{\partial S}
$$

de onde obtém-se:

$$
-\frac{\partial \Psi}{\partial \prod_{\approx}} \underset{\approx}{\dot{\Pi}}-\left(\frac{1}{T}\right) \underset{\approx}{h \operatorname{div} T} \geq 0
$$

Uma vez que:

$$
\underset{\sim}{\operatorname{hivT}} \leq \mathbf{0}
$$

cuja interpretação física é de que o fluxo de calor ocorre do ponto de maior temperatura para o ponto de menor temperatura. Logo, tem-se que:

$$
\frac{\partial \Psi}{\partial \Pi_{\approx}} \dot{\Pi} \leq \mathbf{0}
$$


Observando-se as eqs.(4.63) vê-se que $\sigma$ e $\mathbf{T}$ são variáveis termodinâmicas relacionadas, respectivamente, a $\underset{\approx}{\boldsymbol{\varepsilon}}$ e $\mathbf{S}$. Analogamente, pode-se definir uma variável termodinâmica, relacionada a cada variável interna $\pi_{\mathbf{k}}$, tal que:

$$
-Y_{k}=\frac{\partial \Psi}{\partial \pi_{k}}
$$

Interpretando-se o dano local no concreto como um conjunto de variáveis $\mathbf{D}_{\mathbf{k}}$ e considerando que a energia interna local diminui com o dano, pode-se escrever:

$$
\frac{\partial \Psi}{\partial \pi_{\mathrm{k}}}=\frac{\partial \Psi}{\partial \mathrm{D}_{\mathrm{k}}} \leq \mathbf{0}
$$

Comparando-se as inequações dadas por (4.67) e (4.68), tem-se que:

$$
\dot{\mathrm{D}}_{\mathrm{k}} \geq \mathbf{0}
$$

ou seja, a variável de dano não diminui com o tempo. Esta é a condição necessária para que uma variável que represente o dano seja considerada uma variável termodinâmica interna. Esta condição deverá, então, ser satisfeita toda vez que se derivar equações constitutivas a partir de potenciais termodinâmicos. (BUSSAMRA, 1993).

\subsubsection{Variáveis de Dano}

Seja um elemento de volume representativo de um sólido danificado. Este elemento é considerado de tamanho tal, de forma a ser interpretado como um ponto material do contínuo e suficientemente grande de maneira a conter as imperfeições e o material constitutivo do meio (fig. 4.6). 
Nesta figura $\mathbf{S}$ é a área total de uma seção deste elemento; $\mathbf{S}_{\mathbf{o}}$ é a área com defeitos e $\overrightarrow{\mathbf{n}}$ o versor que define a área $\mathbf{S}$ no ponto. Denominando-se $\overline{\mathbf{S}}$ a área resistente efetiva tem-se, para a área com defeitos $\mathbf{S}_{\mathbf{0}}$, que:

$$
\mathbf{S}_{\mathbf{o}}=\mathbf{S}-\overline{\mathbf{S}}
$$

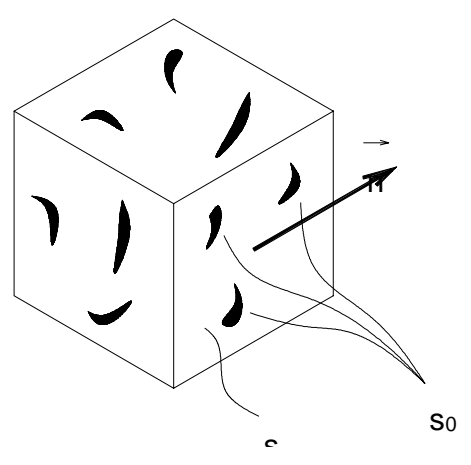

\section{FIGURA 4.6. Elemento de volume danificado}

Uma medida mecânica do dano local, admitindo-se a hipótese de continuidade e representando o caso de dano anisotrópico, é dada por:

$$
D_{n}=\lim _{s \rightarrow 0} \frac{S_{0}}{S}
$$

Como pode ser observado, $\mathbf{0} \leq \mathbf{D}_{\mathbf{n}} \leq \mathbf{1}$. O caso particular de $\mathbf{D}_{\mathbf{n}}=\mathbf{0}$ representa um estado de dano inexistente ou material íntegro; $\mathbf{D}_{\mathbf{n}}=\mathbf{1}$ representa o estado limite local de fraturamento (material totalmente deteriorado).

Uma outra situação possível, é considerar a danificação isotrópica, denominada dano isótropo ou escalar. Neste caso, considera-se que os microdefeitos têm uma distribuição uniforme independente da orientação da normal n. Assim, uma variável escalar caracteriza completamente o estado de dano local e pode ser definido como:

$$
D_{n}=D \quad v \quad \vec{n}
$$




\subsubsection{Deformação Equivalente}

Seja, no caso de dano isótropo, um corpo em equilíbrio estático. Seja $\mathbf{F}$ a força agindo numa seção $\mathbf{S}$ de um elemento de volume e $\mathbf{\sigma}=\mathbf{F} / \mathbf{S}$ a distribuição de tensões que satisfaça a equação de equilíbrio. A variável de dano isótropo é dada por:

$$
\mathbf{D}=\frac{\mathbf{S}_{\mathbf{o}}}{\mathbf{S}}
$$

Considerando-se as eqs.(4.70) e (4.73) pode-se expressar a área efetiva por:

$$
\overline{\mathbf{S}}=\mathbf{S}(\mathbf{1}-\mathbf{D})
$$

e a tensão efetiva, aplicada à parte resistente da seção fica expressa por:

$$
\bar{\sigma}=\frac{F}{\bar{S}}=\frac{\sigma S}{S(1-D)}=\frac{\sigma}{(1-D)}
$$

onde os casos particulares de $\bar{\sigma}=\sigma$ e $\bar{\sigma} \rightarrow \infty$ representam as condições de material íntegro e de um estado limite de deterioração, respectivamente.

Como no caso tridimensional de dano isótropo a relação $\mathbf{S} / \mathbf{S}_{\mathbf{0}}$ independe da normal e o fator (1-D) altera todas as componentes do tensor de tensões $\underset{\boldsymbol{\sigma}}{\boldsymbol{\sigma}}$, tem-se que:

$$
\bar{\sigma}=\underset{\approx}{\sigma}(1-D)
$$

onde $\underset{\approx}{\sigma}$ é o tensor de tensões efetivo.

A hipótese de deformação equivalente dada por LEMAITRE, CHABOCHE (1985) ${ }^{⿴}$ apud ÁLVARES (1993) ${ }^{\text {耳 }}$ desempenha papel fundamental na formulação de uma relação constitutiva para um material danificado tratado como um contínuo. Tal hipótese é dada por: "O mesmo estado de deformação de um material com dano pode

\footnotetext{
${ }^{4}$ LEMAITRE, J.; CHABOCHE, J.C. Mécanique des matériaux solides. Paris, 1985. Dunod Bordas.

${ }^{5}$ ÁLVARES, M. S. Estudo de um modelo de dano para o concreto: formulação, identificação paramétrica e aplicação com o emprego do Método dos Elementos Finitos. Dissertação (Mestrado). São Carlos, 1993. Escola de Engenharia de São Carlos, Universidade de São Paulo.
} 
ser derivado do material íntegro onde a tensão usual é substituída pela tensão efetiva." Esta hipótese é ilustrada na fig. 4.7.

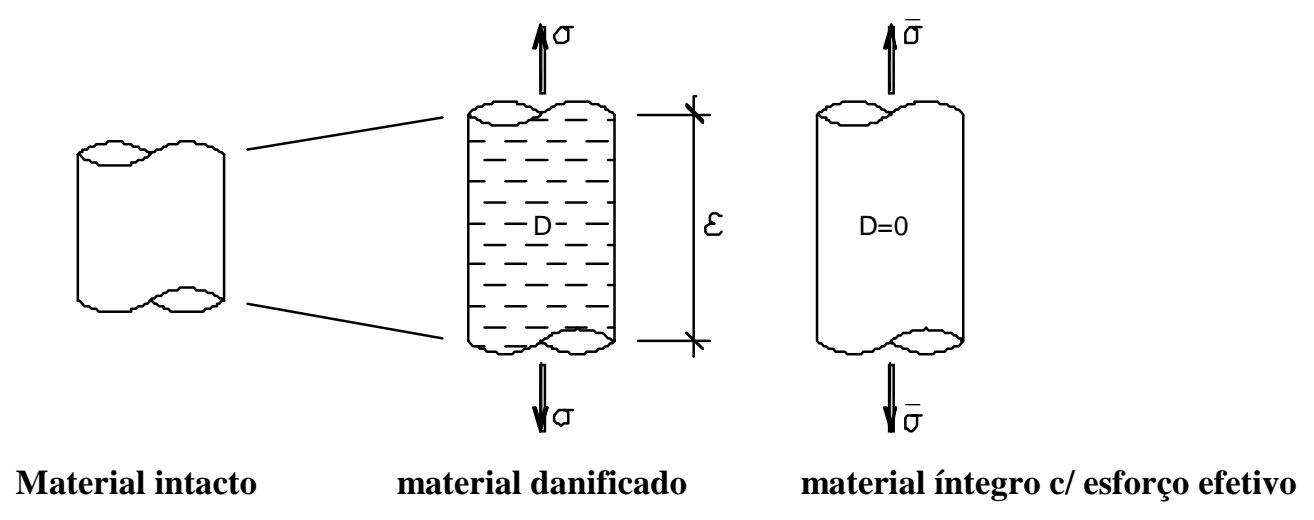

\section{FIGURA 4.7. Hipótese de deformação equivalente}

Com isso, a deformação elástica no material com dano pode ser descrita em função da deformação de um material íntegro de seguinte forma:

$$
\varepsilon=\frac{\bar{\sigma}}{E}=\frac{\sigma}{E(1-D)}=\frac{\sigma}{\bar{E}}
$$

onde $\overline{\mathbf{E}}$ é o módulo de elasticidade do material danificado.

\subsubsection{Modelo Constitutivo de Mazars}

Apresenta-se a seguir o modelo de dano proposto por MAZARS (1984). Este modelo é apropriado para o concreto submetido a um carregamento proporcional crescente sendo, portanto, utilizado no programa computacional ora desenvolvido. 


\section{a) Hipóteses básicas}

As hipóteses básicas nas quais o modelo se fundamenta são:

- Em processo de dano evolutivo, o concreto apresenta comportamento elástico desprezando-se, portanto, as deformações permanentes observadas, numa situação de descarregamento;

- O dano é causado somente pela existência de extensões (alongamentos), que ocorrerão ao longo de uma ou mais direções principais de deformação;

- O dano é isótropo, ou seja, o estado de dano num ponto é representado por uma grandeza escalar;

- Representa-se o dano local por uma variável local escalar $\mathbf{D}(\mathbf{0} \leq \mathbf{D} \leq \mathbf{1})$, cuja evolução ocorre quando for superado um certo valor de referência para o alongamento equivalente.

\section{b) deformações principais}

A relação tensão - deformação, segundo a lei de Hooke, é dada pela eq.(2.7). As componentes de tensão dadas por aquela equação e expressas nas direções principais, podem ser escritas como:

$$
\sigma_{i}=\sigma_{i}^{+}+\sigma_{i}^{-}
$$

$$
\begin{array}{llll}
\text { sendo que: } & \text { se } \sigma_{i}>\mathbf{0}, & \sigma_{i}^{+}=\sigma_{i} ; & \sigma_{i}^{-}=\mathbf{0} \\
& \text { se } \sigma_{i}<0, & \sigma_{i}^{+}=0 ; & \sigma_{i}^{-}=\sigma_{i}
\end{array}
$$

Assim, as deformações $\boldsymbol{\varepsilon}_{\mathbf{T i}}$ devidas às tensões de tração e as deformações $\boldsymbol{\varepsilon}_{\mathbf{C i}}$ devidas às tensões de compressão, em cada direção principal é obtida, segundo a lei de Hooke, por:

$$
\begin{aligned}
& \varepsilon_{\mathrm{Ti}}=\frac{1+v}{E} \sigma_{i}^{+}-\frac{v}{E} \sum_{i=1}^{3} \sigma_{i}^{+} \\
& \varepsilon_{\mathrm{Ci}}=\frac{1+v}{E} \sigma_{i}^{-}-\frac{v}{E} \sum_{i=1}^{3} \sigma_{i}^{-}
\end{aligned}
$$


e a deformação total $\varepsilon_{\mathbf{i}}$ fica expressa por:

$$
\varepsilon_{i}=\varepsilon_{T i}+\varepsilon_{C i}
$$

\section{c) Deformação equivalente $\bar{\varepsilon}$}

Obtidas as deformações principais dadas pela eq.(4.80), obtém-se os alongamentos $\boldsymbol{\varepsilon}_{\mathbf{i}}^{+}$nas direções principais como:

$$
\varepsilon_{i}^{+}=\frac{1}{2}\left(\varepsilon_{i}+\left|\varepsilon_{i}\right|\right)
$$

onde:

$$
\begin{array}{ll}
\varepsilon_{i}^{+}=\varepsilon_{i} & \text { se } \varepsilon_{i}>0 \\
\varepsilon_{i}^{+}=0 & \text { se } \varepsilon_{i} \leq 0
\end{array}
$$

Com os valores dos alongamentos $\varepsilon_{\mathbf{i}}^{+}$, a deformação equivalente $\bar{\varepsilon}$, dada por:

$$
\bar{\varepsilon}=\sqrt{\left(\varepsilon_{1}^{+}\right)^{2}+\left(\varepsilon_{2}^{+}\right)^{2}+\left(\varepsilon_{3}^{+}\right)^{2}}
$$

traduz o estado local de extensão do material. 


\section{d) Critério de dano}

Admite-se que o processo de danificação do material tem início quando a deformação equivalente $\overline{\boldsymbol{\varepsilon}}$ atinge o valor $\overline{\boldsymbol{\varepsilon}}=\boldsymbol{\varepsilon}_{\mathbf{d o}}$ onde $\boldsymbol{\varepsilon}_{\mathbf{d o}}$ é a deformação correspondente ao máximo esforço num teste uniaxial de tração, como ilustra a fig.(4.8).

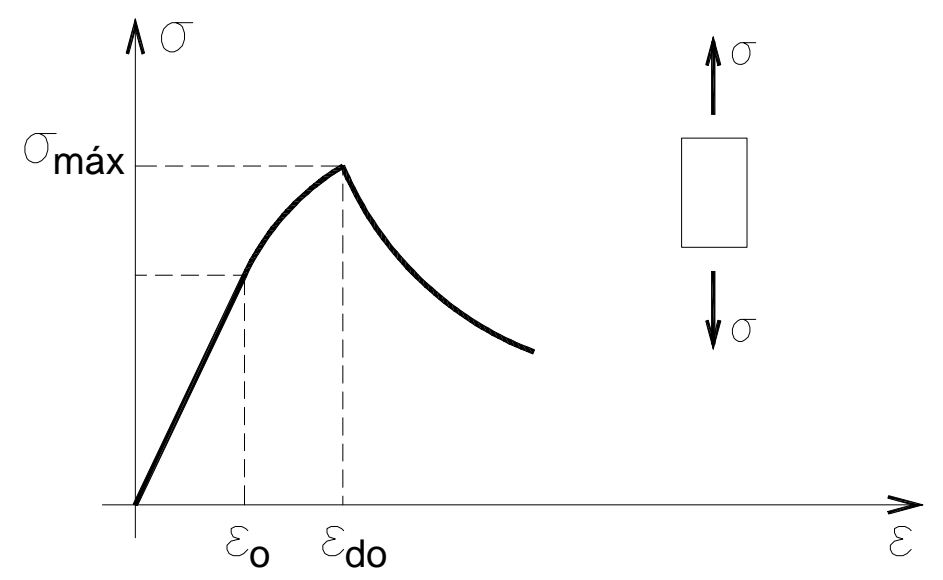

FIGURA 4.8. Diagrama $\sigma$ x $\varepsilon$ - teste uniaxial de tração: representação de $\varepsilon_{\mathrm{do}}$ •

Assim, o critério de dano fica expresso por:

$$
\mathbf{f}(\bar{\varepsilon}, \mathbf{D})=\bar{\varepsilon}-\mathbf{S}(\mathbf{D}) \leq \mathbf{0}
$$

onde $\mathbf{S}_{\mathbf{o}}=\mathbf{S}(\mathbf{D}=\mathbf{0})=\boldsymbol{\varepsilon}_{\mathbf{d o}}$ é o limite elástico inicial. Fazendo-se $\mathbf{f}(\overline{\boldsymbol{\varepsilon}}, \mathbf{D})=\mathbf{0}$ e substituindo-se o valor de $\boldsymbol{\varepsilon}_{\mathrm{do}}$ dado pela eq.(4.82), obtém-se a função $\mathbf{S}(\mathbf{D})$, ou seja:

$$
\mathbf{S}(\mathbf{D})=\bar{\varepsilon}=\sqrt{\left(\varepsilon_{1}^{+}\right)^{2}+\left(\varepsilon_{2}^{+}\right)^{2}+\left(\varepsilon_{3}^{+}\right)^{2}}
$$

Considerando-se o espaço das tensões principais, a função $\mathbf{S}(\mathbf{D})$ representa uma superfície de um quarto de uma esfera de raio $\mathbf{S ( D ) , ~ c o m o ~ m o s t r a ~ a ~ f i g . ( 4 . 9 ) . ~}$ 


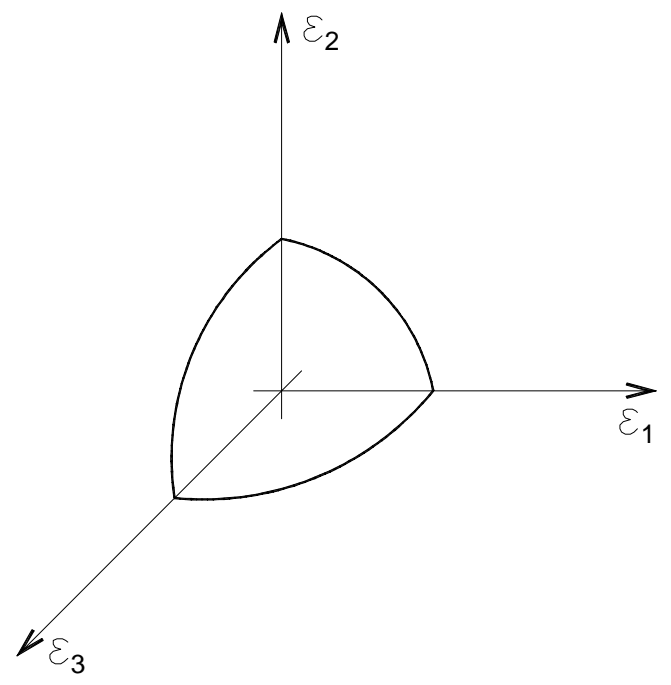

FIGURA 4.9. Superfície de ruptura no espaço das deformações principais.

Um ponto dentro da superfície significa que o estado de alongamento $\bar{\varepsilon}$ é menor que o limite elástico $\mathbf{S}(\mathbf{D})$, não havendo evolução do dano. Um ponto fora da superfície, ou seja, $\overline{\boldsymbol{\varepsilon}}$ maior que $\mathbf{S}(\mathbf{D})$, caracteriza evolução do dano.

A forma da superfície inicial $\mathbf{f}$, no espaço das tensões principais $\boldsymbol{\sigma}_{\mathbf{1}}$ e $\boldsymbol{\sigma}_{\mathbf{2}}$, está representada na fig.4.10. Compara-se, na mesma figura, esta superfície com a superfície obtida por KUPFER (1969). Representa-se também a superfície corrigida, que consiste em multiplicar $\overline{\boldsymbol{\varepsilon}}$ por um coeficiente $\boldsymbol{\gamma} \leq \mathbf{1}$. Assim,:

$\bar{\varepsilon}_{\text {corrig }}=\gamma\left(\sigma_{\mathbf{i}}\right) \bar{\varepsilon}$
onde: $\quad \gamma\left(\sigma_{i}\right)=\frac{\sqrt{\sum_{i}\left(\sigma_{i}^{-}\right)^{2}}}{\sum_{i} \sigma_{i}}$

sendo $\sigma_{\mathbf{i}}^{-}$a parte negativa da tensão principal $\boldsymbol{\sigma}_{\mathbf{i}}$. 


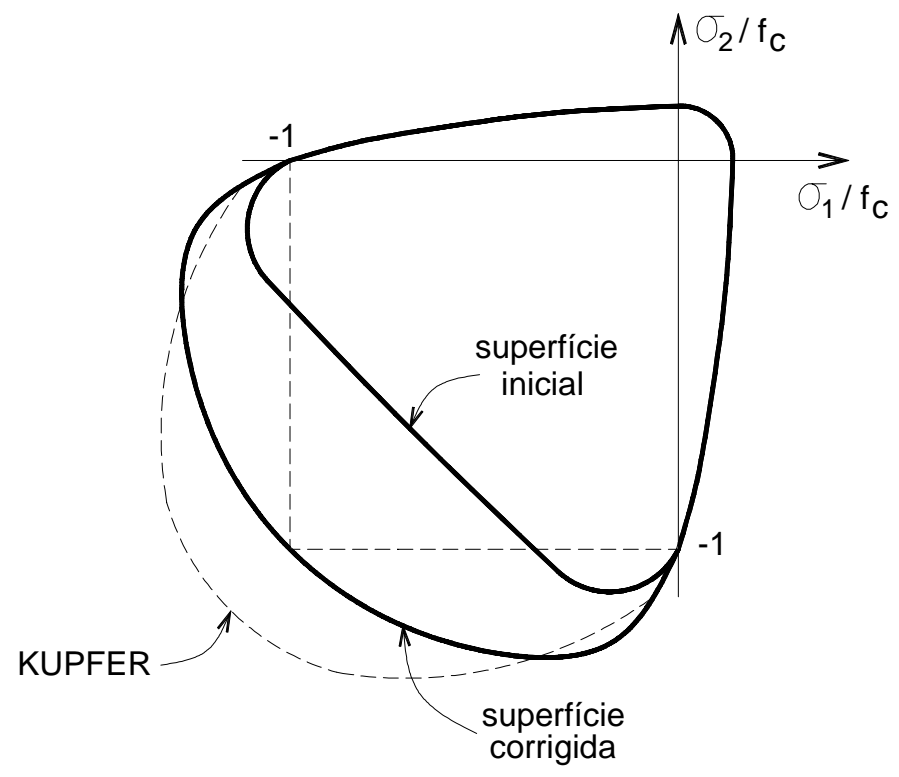

FIGURA 4.10. Superfícies de ruptura no espaço das tensões principais $\sigma_{1}$ e $\sigma_{2}$.

A lei de evolução do dano $\mathbf{D}$, que atende aos princípios da termodinâmica dos processos irreversíveis é dada por:

$\dot{\mathbf{D}}=\mathbf{0} \quad$ se $\quad \mathbf{f}(\bar{\varepsilon}, \mathbf{D}) \leq \mathbf{0} \quad$ e $\quad \dot{\mathbf{f}}(\overline{\boldsymbol{\varepsilon}}, \mathbf{D})<\mathbf{0}$ (caso de descarregamento)

$\dot{\mathbf{D}}>\mathbf{0} \quad$ se $\quad \mathbf{f}(\bar{\varepsilon}, \mathbf{D})=\mathbf{0} \quad$ e $\quad \dot{\mathbf{f}}(\bar{\varepsilon}, \mathbf{D})=\mathbf{0}$ (caso de carregamento)

Se $\dot{\mathbf{D}}=\mathbf{0}$ e $\quad \dot{\mathbf{f}}(\overline{\boldsymbol{\varepsilon}}, \mathbf{D})=\mathbf{0}$ tem-se o caso de carregamento neutro.

Não se admite $\mathbf{f}>\mathbf{0}$; quando isso ocorre, há evolução da variável de dado e o novo limite elástico passa a ser $\mathbf{S}(\mathbf{D})$, definido pelo estado de alongamento atual.

\section{e) Determinação da variável de dano}

Dado o comportamento não simétrico do concreto à tração e à compressão uniaxiais, segue-se uma diferença no processo de evolução da microfissuração. Enquanto em tração as microfissuras se desenvolvem perpendicularmente à direção do esforço, em compressão elas se desenvolvem paralelamente ao esforço. Isto leva à definição de duas variáveis escalares de dano: uma para o caso de tração, $\mathbf{D}_{\mathbf{T}}$, e outra 
para o caso de compressão, $\mathbf{D}_{\mathbf{C}}$. O processo de evolução de dano para estes dois casos é governado por leis independentes.

Como no caso multiaxial cada componente de tensão pode contribuir para a evolução da danificação do material, a variável de dano fica determinada por uma combinação linear de $\mathbf{D}_{\mathbf{T}}$ e $\mathbf{D}_{\mathbf{C}}$, definidas de modo a bem representar as curvas experimentais de tração e compressão uniaxiais, para o caso de carregamento proporcional. Logo:

$$
\mathrm{D}=\alpha_{\mathrm{T}} \mathbf{D}_{\mathrm{T}}+\alpha_{\mathrm{C}} \mathbf{D}_{\mathrm{C}}
$$

onde: $\quad 0 \leq \alpha_{\mathrm{T}} \leq 1 ; \quad 0 \leq \alpha_{\mathrm{C}} \leq 1 \quad$ e $\quad \alpha_{\mathrm{T}}+\alpha_{\mathrm{C}}=1$

As expressões para $\mathbf{D}_{\mathbf{T}}$ e $\mathbf{D}_{\mathbf{C}}$, são dadas em função da deformação equivalente $\overline{\boldsymbol{\varepsilon}}$, por:

$$
\begin{aligned}
& D_{\mathrm{T}}(\bar{\varepsilon})=1-\frac{\varepsilon_{\mathrm{do}}\left(1-\mathbf{A}_{\mathrm{T}}\right)}{\bar{\varepsilon}}-\frac{\mathbf{A}_{\mathrm{T}}}{\exp \left[\mathrm{B}_{\mathrm{T}}\left(\bar{\varepsilon}-\varepsilon_{\mathrm{do}}\right)\right]} \\
& \mathrm{D}_{\mathrm{C}}(\bar{\varepsilon})=1-\frac{\varepsilon_{\mathrm{do}}\left(1-\mathbf{A}_{\mathrm{C}}\right)}{\bar{\varepsilon}}-\frac{\mathbf{A}_{\mathrm{C}}}{\exp \left[\mathrm{B}_{\mathrm{C}}\left(\bar{\varepsilon}-\varepsilon_{\mathrm{do}}\right)\right]}
\end{aligned}
$$

sendo:

$$
\alpha_{\mathrm{T}}=\frac{\sum_{\mathrm{i}=1}^{3} \varepsilon_{\mathrm{Ti}}^{+}}{\varepsilon_{\mathrm{v}}^{+}} ; \quad \alpha_{\mathrm{C}}=\frac{\sum_{\mathrm{i}=1}^{3} \varepsilon_{\mathrm{Ci}}^{+}}{\varepsilon_{\mathrm{v}}^{+}}
$$

e:

$$
\varepsilon_{\mathrm{v}}^{+}=\sum_{\mathrm{i}=1}^{3} \varepsilon_{\mathrm{Ti}}^{+}+\varepsilon_{\mathrm{Ci}}^{+}
$$

sendo $\boldsymbol{\varepsilon}_{\mathbf{T}}^{+}$e $\boldsymbol{\varepsilon}_{\mathrm{C}}^{+}$as componentes positivas dos tensores $\boldsymbol{\varepsilon}_{\mathbf{T}}$ e $\boldsymbol{\varepsilon}_{\mathbf{C}}$, os quais são obtidos pelas expressões (4.79.a,b); $\boldsymbol{\varepsilon}_{\mathbf{T}}^{+}$e $\boldsymbol{\varepsilon}_{\mathbf{C}}^{+}$são determinadas considerando-se a eq.(4.81). 
$\mathbf{A}_{\mathrm{T}}, \mathbf{B}_{\mathrm{T}}, \mathbf{A}_{\mathrm{C}}, \mathbf{B}_{\mathrm{C}} \mathbf{e} \boldsymbol{\varepsilon}_{\mathrm{do}}$ são parâmetros característicos do material.

ÁLVARES (1993) descreve a metodologia de identificação paramétrica, tendo obtido os seguintes resultados, para corpos de prova com resistência média aos 21 dias de 25,51 Mpa:

- Módulo de elasticidade do concreto: $\mathbf{E}=29200 \mathrm{MPa}$;

- Parâmetros relativos a tração:

$$
\mathrm{A}_{\mathrm{T}}=\mathbf{0 . 9 9 5 ;} \quad \mathrm{B}_{\mathrm{T}}=\mathbf{8 0 0 0} ; \quad \varepsilon_{\mathrm{do}}=\mathbf{0 . 0 0 0 0 7}
$$

- Parâmetros relativos à compressão:

$$
\mathrm{A}_{\mathrm{C}}=\mathbf{0 . 8 5} ; \quad \mathrm{B}_{\mathrm{C}}=\mathbf{1 6 2 0}
$$

Nas figs. 4.11 e 4.12 mostram-se como o modelo apresentado reproduz aproximadamente, o comportamento real.

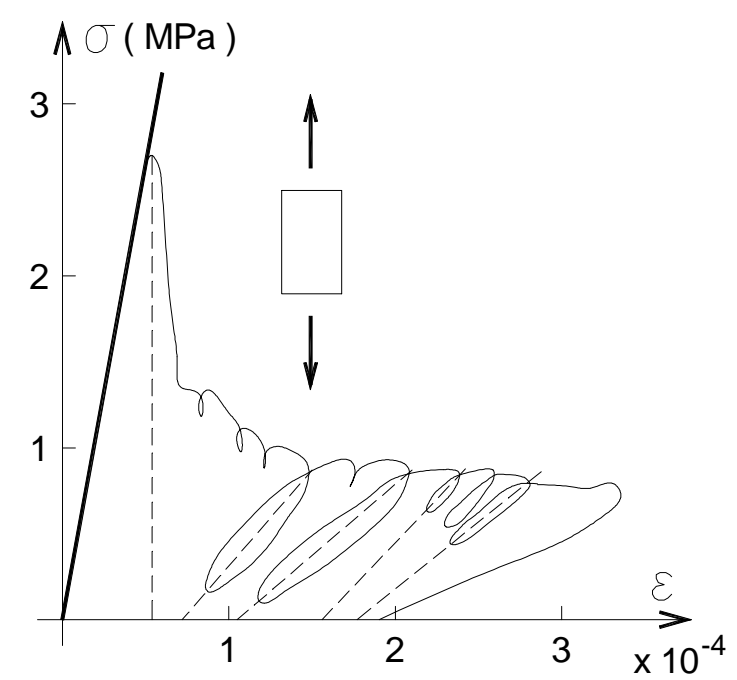

a) Curva experimental

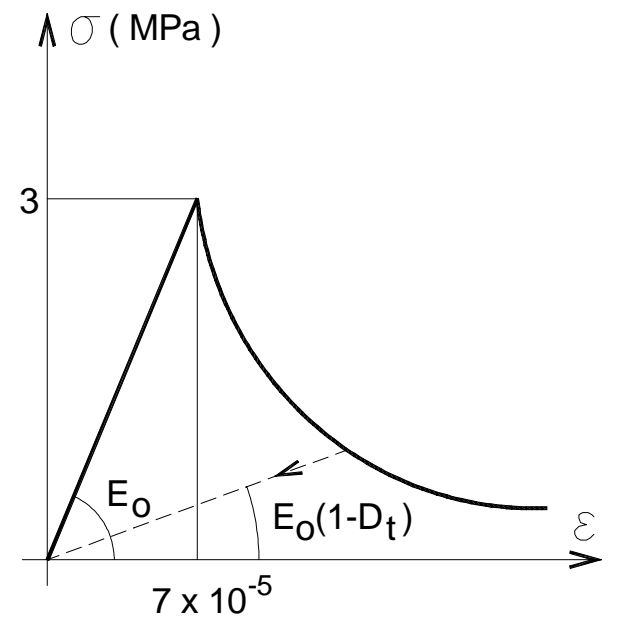

b)relação constitutiva

FIGURA 4.11. tração uniaxial (PEREGO, 1990) 


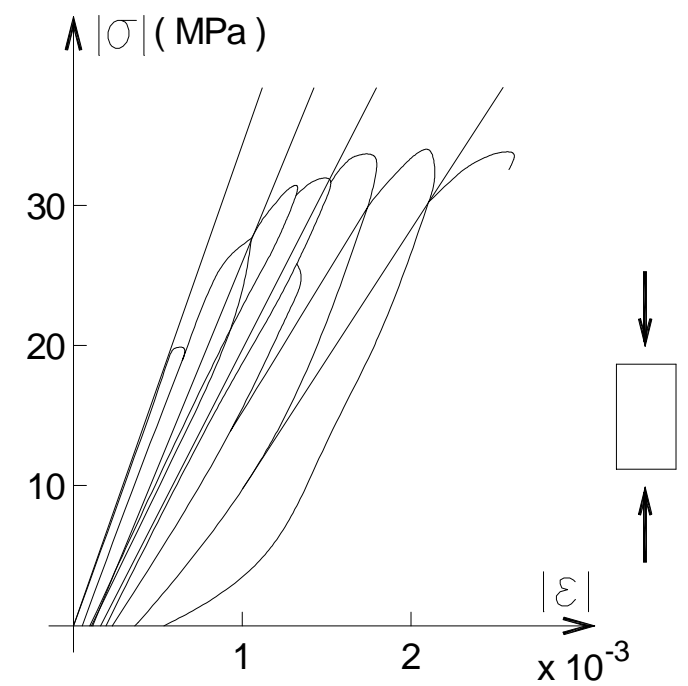

a) curva experimental

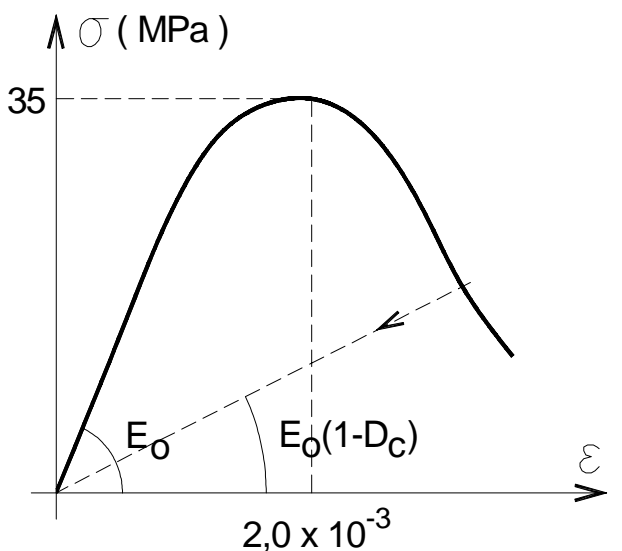

b) relação constitutiva

FIGURA 4.12. compressão uniaxial (PEREGO, 1990) 


\section{CAPÍt́tulO V}

\section{SOLUÇÃO DO PROBLEMA NÃO LINEAR}

\subsection{INTRODUÇÃO}

ZIENKIEWICS et al.(1969) apresentaram um processo denominado processo das tensões iniciais, voltado para o método dos elementos finitos, no qual a solução de um problema não-linear é obtida através de incrementos sucessivos de carregamento.

Assim, baseado no processo referido no parágrafo anterior e com as formulações para placas com campo de momentos iniciais e placas enrijecidas, utilizando-se as teorias de análise não linear apresentadas no capítulo IV, é possível obter-se a solução numérica para o problema de pavimentos, como é visto nos próximos itens.

Dada a relação tensão - deformação não linear, tanto do concreto como do aço, o carregamento sobre a placa é aplicado em incrementos. Utiliza-se, então, um algoritmo incremental - iterativo, sem atualização das matrizes envolvidas, semelhante àquele originalmente apresentado por OWEN \& HINTON (1980) para o Método dos Elementos Finitos e adaptado para o Método dos Elementos de Contorno por RIBEIRO (1992).

O problema é resolvido uma vez para o valor total do carregamento, admitindo-se comportamento elástico linear. Os momentos são, então, calculados para os pontos da placa através da eq.(2.100). Obtém-se, assim, o vetor de momentos elásticos $\underset{\approx}{\mathbf{M}}$ para o carregamento atuante. Não ocorrendo, para o carregamento real 
atuante, efeitos de campos de momentos iniciais, obtém-se a solução elástica para os momentos por:

$$
\underset{\approx}{\mathbf{M}^{\mathrm{e}}}=\underset{\mathrm{N}}{\mathrm{N}}
$$

O valor de incremento de carga é tomado como uma fração do carregamento para o qual foi determinada a solução elástica $\underset{\approx}{\mathbf{M}^{\mathbf{e}}}$. Assim, para um incremento de carga genérico $\mathbf{m}$, a resposta elástica em termos de momentos $\left(\Delta \mathbf{M}^{\mathbf{e}}\right)_{\mathbf{m}}$ é:

$$
\left.\underset{\approx}{\left(\Delta \mathbf{M}^{\mathrm{e}}\right.}\right)_{\mathrm{m}}=\beta_{\mathrm{m}} \underset{\approx}{\mathbf{M}^{\mathrm{e}}}
$$

onde $\beta_{m}$ é o fator de carga.

Se o incremento de momentos não for totalmente elástico, processam-se novas iterações, até que se encontre um estado de momentos internos aproximadamente igual ao estado de momentos externos. Este processo iterativo é abordado no item 5.5.

Para as demais iterações do incremento, o incremento de momentos elásticos é obtido por:

$$
\left.\underset{\approx}{\left(\Delta \mathbf{M}^{\mathrm{e}}\right.}\right)_{\mathbf{m}}=\underset{\approx}{\mathbf{T}} \underset{\approx}{\Delta \mathbf{M}^{\mathbf{0}}}
$$

sendo $\underset{\approx}{\mathbf{T}}$ determinado pela eq.(2.99) e $\underset{\approx}{\Delta \mathbf{M}^{\mathbf{0}}}$, o vetor de momentos residuais, interpretado como um campo de momentos iniciais.

O critério de convergência deste processo é dado pela relação entre o módulo de incremento de alongamento equivalente $\Delta \overline{\boldsymbol{\varepsilon}}$ e o limite elástico $\mathbf{S}(\mathbf{D})$, ou pela relação entre o módulo de incremento de tensão efetiva, suposto elástico, e a tensão de escoamento $\boldsymbol{\sigma}_{\mathbf{y}}^{\mathbf{n}}$ da iteração $\mathbf{n}$ em consideração, para pontos pertencentes ao concreto ou à armadura longitudinal, respectivamente. Assim:

$$
\frac{|\Delta \bar{\varepsilon}|}{\mathrm{S}(\mathrm{D})} \leq \text { toler } \quad \text { ou } \quad \frac{|\Delta \bar{\sigma}|}{\sigma_{\mathrm{y}}^{\mathrm{n}}} \leq \text { toler }
$$

sendo toler a tolerância de cálculo. 
Quanto aos modelos constitutivos, a laje é dividida em camadas de concreto e aço, conforme descrito a seguir. Para a verificação do comportamento do concreto, adotou-se o modelo de dano de Mazars considerando-se, também, as deformações associadas ao cisalhamento. Para a armadura longitudinal foi adotado um modelo elastoplástico uniaxial.

\subsection{MODELO ESTRATIFICADO}

Este modelo permite acompanhar o processo de danificação ao longo da espessura da placa. Cada camada pode ter espessura e propriedades diferentes. Em cada camada, porém, as propriedades são consideradas constantes.

Considere-se que, para um determinado nível de carregamento, a distribuição real de tensões em uma seção da placa seja aquela indicada na fig.5.1.b. Com a aproximação adotada, admite-se que cada camada possua um ponto de tensão no seu plano médio. Calculam-se as componentes de tensão para estes pontos as quais são consideradas constantes ao longo da espessura $\mathbf{h}_{\mathbf{i}}$ de cada camada. Assim, a distribuição de tensões é discretizada como indica a fig.5.1.c.

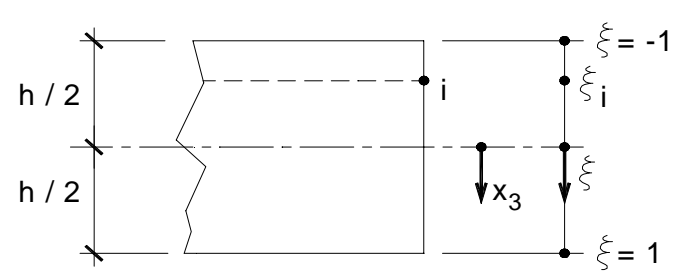

a) seção transversal

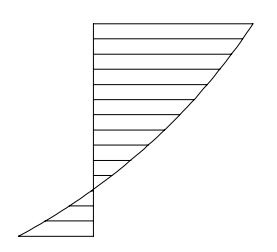

b) distribuição real de tensões

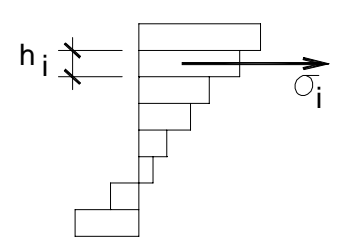

c) distribuição em camadas

\section{FIGURA 5.1. Distribuição de tensões em uma seção da placa}

Assim, em função do estado de deformação, verificam-se as condições de danificação em cada ponto e os momentos resultantes nas seções são obtidos pela integração das respectivas componentes de tensão ao longo da espessura.

Num modelo que melhor representa a realidade do concreto armado, a espessura é dividida em camadas de concreto e as armaduras são distribuídas em camadas de espessuras equivalentes, como mostra a fig.5.2. Entende-se por espessura 
equivalente de uma camada de aço, aquela cuja área correspondente da armadura permanece inalterada.
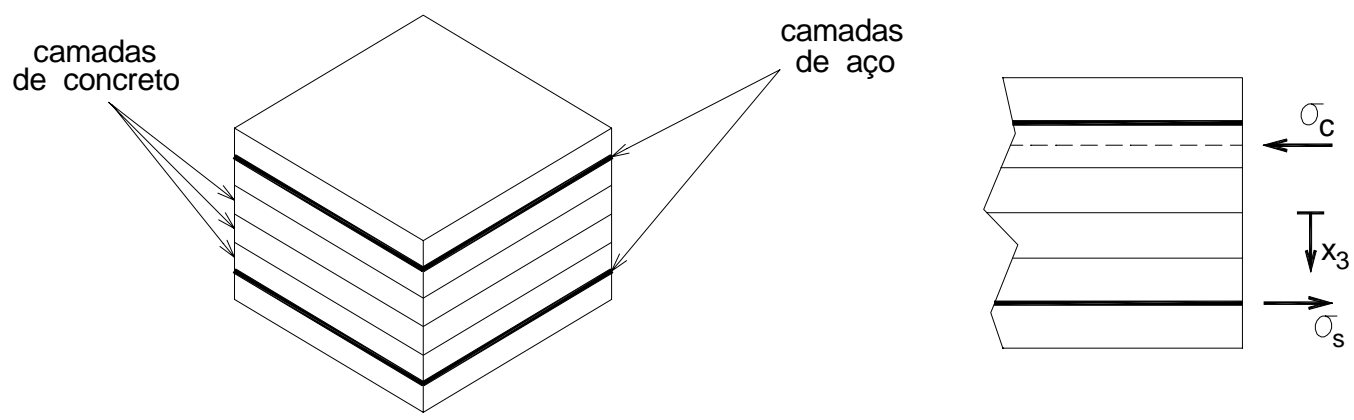

\section{FIGURA 5.2. Modelo estratificado para o concreto armado}

Assim, os momentos resultantes na seção transversal e os esforços cortantes no concreto são dados, respectivamente, por:

$$
\begin{aligned}
& M_{\alpha \beta}=\int_{-h / 2}^{h / 2} \sigma_{\alpha \beta}^{i} x_{3} d x_{3}+\sum_{i=1}^{\text {ns }} \sigma_{\alpha \beta}^{\text {is }} \delta_{\alpha \beta} A_{s}^{\text {is }} x_{3}^{\text {is }} \\
& Q_{\alpha 3}=\int_{-h / 2}^{h / 2} \tau_{\alpha 3}^{i} d x_{3}
\end{aligned}
$$

calculados numericamente pela quadratura de Gauss,

onde:

$\sigma_{\alpha \beta}^{\mathrm{i}}, \tau_{\alpha 3}^{\mathrm{i}}$ são as componentes de tensão normal e tangencial, respectivamente, na camada i;

$\mathbf{A}_{\mathbf{S}}^{\text {is }}, \mathbf{x}_{3}^{\text {is }}$ são a área da armadura e a posição da camada is, respectivamente; ns é o número de camadas de armadura. 
Os pontos ao longo da espessura são definidos em função da coordenada homogênea $\xi$, como indica a fig.5.1.a e são coincidentes com os pontos utilizados na integração numérica. Assim, a relação entre as coordenadas de um ponto será:

$$
\begin{gathered}
x_{3}=\xi \frac{h}{2} \\
d x_{3}=\frac{h}{2} d \xi
\end{gathered}
$$

Fazendo-se a mudança da coordenada $\mathbf{x}_{3}$ para a coordenada homogênea $\boldsymbol{\xi}$, pode-se escrever:

$$
\begin{aligned}
& \mathbf{M}_{\alpha \beta}=\frac{\mathbf{h}^{2}}{4} \sum_{\mathrm{ig}=1}^{\mathrm{ng}} \sigma_{\alpha \beta}^{\mathrm{i}(\mathrm{ig})} \xi_{\mathrm{ig}} \mathbf{W}_{\mathrm{ig}}+\sum_{\mathrm{i}=1}^{\mathrm{ns}} \sigma_{\alpha \beta}^{\mathrm{is}} \delta_{\alpha \beta} \mathbf{A}_{\mathrm{s}}^{\mathrm{is}} \mathbf{x}_{3}^{\mathrm{is}} \\
& \mathbf{Q}_{\alpha 3}=\frac{\mathbf{h}}{2} \sum_{\mathrm{ig}=1}^{\mathrm{ng}} \tau_{\alpha 3}^{\mathrm{i}(\mathrm{ig})} \mathbf{W}_{\mathrm{ig}}
\end{aligned}
$$

Observe-se a necessidade de um número adequado de camadas, a fim de que se obtenha uma boa discretização das tensões e esforços resultantes satisfatórios.

\subsection{PROCEDIMENTO PARA ANULAR O ESFORÇO NORMAL}

Para elementos de concreto armado submetidos à flexão simples, tem-se a posição da linha ou superfície neutra não coincidente com linha ou superfície média da peça. Assim, para que se tenha uma avaliação mais realista das tensões e deformações ao longo da seção transversal, há que se determinar a posição correta da linha neutra (L.N.). Isto é feito impondo-se a condição de esforço normal nulo.

Ao se alterar a posição da L.N. na direção $\mathbf{x}_{\mathbf{1}}$ alteram-se, também, as posições da L.N. nas direções $\mathbf{x}_{\mathbf{2}}$ e $\mathbf{x}_{\mathbf{1 2}}$. Assim, utiliza-se um processo iterativo que consiste em anular o esforço normal na direção $\mathbf{x}_{1}$ e estimando-se a posição da L.N. em $\mathbf{x}_{2}$; novamente verifica-se o valor do esforço normal na direção $\mathbf{x}_{\mathbf{1}}$. Repete-se o processo até que se tenha esforço normal nulo nestas duas direções. A seguir, estima-se a 
posição da L.N. na direção $\mathbf{x}_{12}$, verificando-se o valor do esforço normal nas direções $\mathbf{x}_{\mathbf{1}}$ e $\mathbf{x}_{\mathbf{2}}$. Repete-se o processo até que se tenha esforço normal nulo nas três direções.

\subsubsection{Nova posição da linha neutra}

A estimativa da nova posição $\mathbf{Z}_{\boldsymbol{\alpha} \boldsymbol{\beta}}$ da L.N. na direção $\alpha \beta$ é feita por interpolação linear em função dos valores das normais $\mathbf{N}_{\alpha \beta}^{\mathbf{1}}, \mathbf{N}_{\alpha \boldsymbol{\beta}}^{\mathbf{2}}$ calculados previamente para as posições $\mathbf{Z}_{\alpha \beta}^{1}, \mathbf{Z}_{\alpha \beta}^{2}$. Como se verifica à partir da fig. 5.3., temse que:

$$
Z_{\alpha \beta}=Z_{\alpha \beta}^{2}+N_{\alpha \beta}^{2} \frac{Z_{\alpha \beta}^{1}-Z_{\alpha \beta}^{2}}{N_{\alpha \beta}^{2}-N_{\alpha \beta}^{1}}
$$

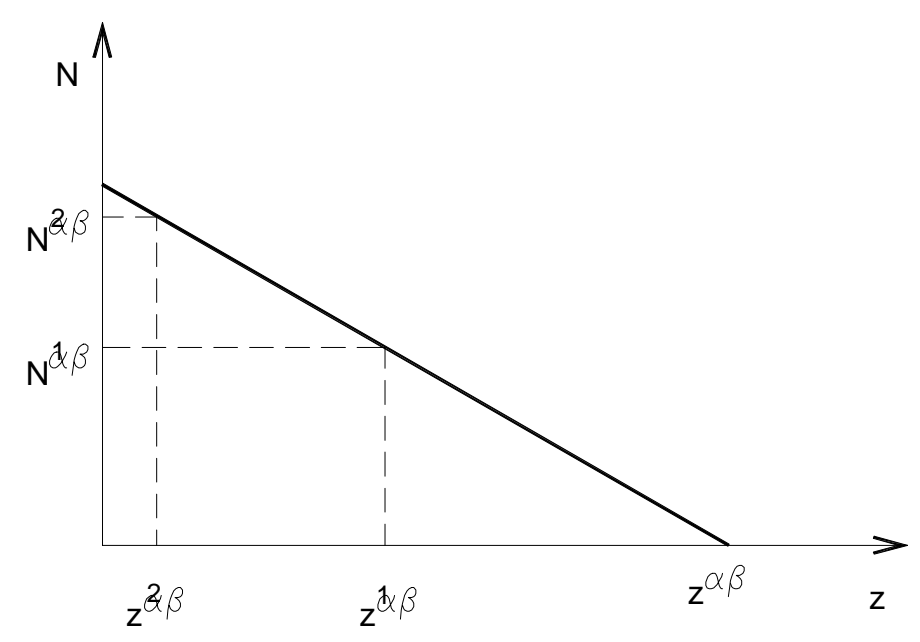

FIGURA 5.3. Estimativa da nova posição da L.N.

Como na primeira estimativa de $\mathbf{Z}_{\alpha \beta}$ não se tem o segundo ponto $\mathbf{Z}_{\alpha \beta}^{\mathbf{2}}$ para se proceder a interpolação, adota-se o mesmo de tal forma que a seção fique inteiramente comprimida. Obtém-se o valor da normal $\mathbf{N}_{\alpha \beta}$ relativo a esta posição. Assim, a partir da segunda estimativa, ter-se-ão três valores para as normais: $\mathbf{N}_{\boldsymbol{\alpha} \boldsymbol{\beta}}^{\mathbf{1}} \mathrm{e}$ $\mathbf{N}_{\alpha \boldsymbol{\beta}}^{\mathbf{2}}$ da interpolação anterior e $\mathbf{N}_{\boldsymbol{\alpha} \boldsymbol{\beta}}$ da interpolação atual. Para a próxima interpolação tem-se: $\mathbf{N}_{\alpha \beta}^{2}=\mathbf{N}_{\alpha \beta}$ e para $\mathbf{N}_{\alpha \beta}^{1}$ tem-se: 


$$
\mathbf{N}_{\alpha \beta}^{1}=\mathbf{N}_{\alpha \beta}^{\prime 2} \text { se }\left|\mathbf{Z}_{\alpha \beta}^{\prime 2}-\mathbf{Z}_{\alpha \beta}\right|<\left|\mathbf{Z}_{\alpha \beta}^{\prime 1}-\mathbf{Z}_{\alpha \beta}\right|
$$

ou:

$$
\mathbf{N}_{\alpha \beta}^{1}=\mathbf{N}_{\alpha \beta}^{\prime 1} \text { se }\left|\mathbf{Z}_{\alpha \beta}^{\prime 1}-\mathbf{Z}_{\alpha \beta}\right|<\left|\mathbf{Z}_{\alpha \beta}^{\prime 2}-\mathbf{Z}_{\alpha \beta}\right|
$$

Seja a fig.5.4. Considerando-se o processo incremental iterativo, no início da iteração n, a posição do ponto $\mathrm{G}$ em relação à L.N. atual é dada por:

$$
Z_{\alpha \beta}^{o(G)}=\frac{\varepsilon_{\alpha \beta}^{n}}{w_{, \alpha \beta}^{n}}
$$

em que $\mathbf{w}_{, \alpha \beta}^{\mathbf{n}}$ é o vetor das curvaturas.

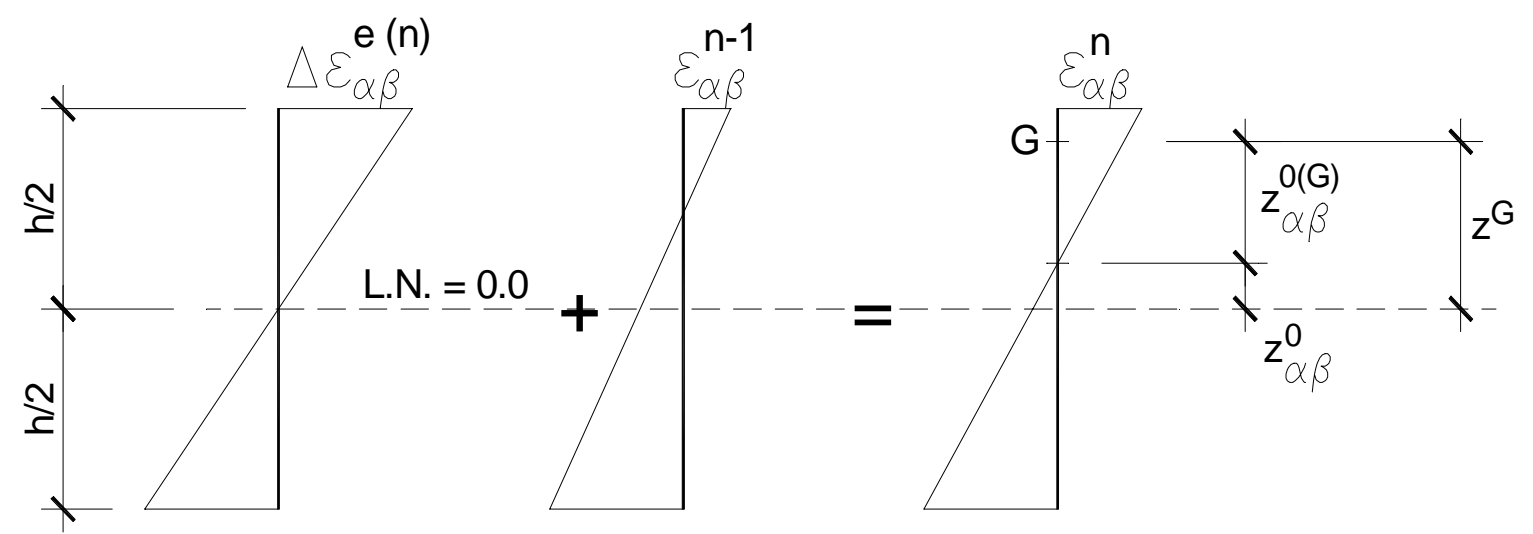

FIGURA 5.4. Distribuição das deformações ao longo da seção transversal

A posição do ponto $\mathbf{G}$ em relação à L.N. na posição 0.0 é dada por:

$$
\mathbf{Z}^{\mathbf{G}}=\frac{\mathbf{h}_{\mathbf{2}}}{\mathbf{2}} \xi_{\mathrm{ig}}
$$

Assim, a posição inicial da L.N. fica expressa por: 


$$
\mathbf{Z}_{\alpha \beta}^{\mathbf{o}}=\mathbf{Z}^{\mathbf{G}}-\mathbf{Z}_{\alpha \beta}^{\mathbf{o}(\mathbf{G})}
$$

\subsubsection{Incrementos de deformação e de tensão}

O incremento de deformação $\Delta \varepsilon_{\alpha \beta}^{\mathbf{L N}}$ relativo à mudança da L.N. é dado por:

$$
\Delta \varepsilon_{\alpha \beta}^{\mathrm{LN}}=\left(\mathbf{Z}_{\alpha \beta}^{\mathbf{0}}-\mathbf{Z}_{\alpha \beta}\right) \mathbf{w}_{, \alpha \beta}
$$

sendo $\mathbf{Z}_{\boldsymbol{\alpha} \beta}^{\mathbf{o}}$ determinado segundo a eq.(5.12).

$O$ vetor das deformações totais, $\varepsilon_{\alpha \beta}^{\mathbf{n}}$, é obtido, como mostra a fig.(5.5), somando-se os incrementos de deformações $\Delta \boldsymbol{\varepsilon}_{\alpha \boldsymbol{\beta}}^{\mathbf{L N}}$ ao incremento de deformações elástico, $\Delta \varepsilon_{\alpha \beta}^{\mathrm{e}}$, devido ao carregamento, acrescido do vetor das deformações verdadeiras, $\boldsymbol{\varepsilon}_{\alpha \beta}^{\mathrm{n}-1}$, da iteração anterior.

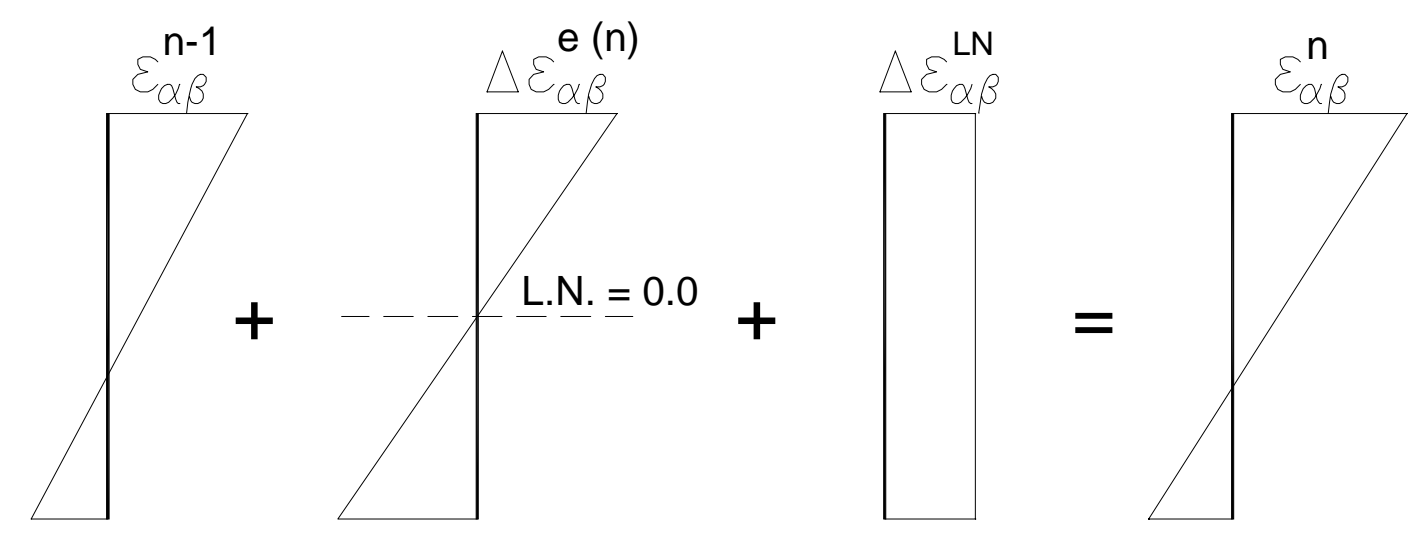

\section{FIGURA 5.5. Incrementos de deformações e deformações totais}

Obtidas as deformações totais, calculam-se as respectivas componentes de tensão elástica. Para os pontos de Gauss, utilizam-se as eqs.(2.7). Para as armaduras, os incrementos de tensão devidos à mudança na posição da L.N. são obtidos por:

$$
\Delta \sigma_{\alpha \beta}^{S(L N)}=E_{S} \Delta \varepsilon_{\alpha \beta}^{L N} \delta_{\alpha \beta}
$$


Com estes valores, verificam-se os modelos constitutivos em todos os pontos ao longo da espessura e determina-se o valor da nova normal resultante obtido, através da integração numérica, por:

$$
N_{\text {C.A. }}=\frac{h}{2} \sum_{i g=1}^{n g} \sigma_{\alpha \beta}^{i(i g)} W_{i g}+\sum_{i=1}^{n s} \sigma_{\alpha \beta}^{i s} \delta_{\alpha \beta} A_{s}^{\text {is }}
$$

\subsubsection{Tolerância}

A posição da L.N. numa determinada direção será considerada correta para valores do esforço normal que satisfaçam a:

$$
\mathbf{N}_{\text {C.A. }} \leq \text { tol. } \mathbf{f}_{\mathrm{ck}} \cdot \mathbf{h}
$$

onde tol é a tolerância de cálculo e $\mathbf{f}_{\mathbf{c k}}$ a resistência característica à compressão do concreto.

\subsection{CONSIDERAÇÃO DAS TENSÕES TANGENCIAIS}

$\mathrm{Na}$ análise de lajes cogumelos, verifica-se uma influência significativa das tensões tangenciais ou de cisalhamento. O modelo aqui utilizado para a consideração destas tensões é baseado na idealização da treliça clássica de Ritter e Mörsch para vigas de concreto armado.

As solicitações tangenciais são absorvidas pelo concreto e pelas armaduras transversais, no caso, estribos a $90^{\circ}$ em relação à superfície média da laje. A proposta aqui, é de um modelo linear de análise. A parcela do esforço cortante resistida pelo concreto fica determinada por:

$$
\mathbf{V}_{\text {conc }}=\frac{\mathbf{h}}{2} \sum_{\mathrm{ig}=1}^{\mathrm{ng}} \tau^{\mathrm{i}(\mathrm{ig})}(\xi) \mathrm{W}_{\mathrm{ig}}
$$

sendo $\tau^{\mathrm{i}(\mathbf{i g})}(\xi)$ a tensão de cisalhamento atuante na camada $\mathbf{i}$ e determinada segundo o critério de dano adotado.

A parcela resistida pela armadura, $\mathrm{V}_{\text {aço }}$, é dada pela diferença: 


$$
\mathbf{V}_{\mathrm{aco}}=\mathrm{V}_{\mathrm{apl}}-\mathrm{V}_{\text {conc }}
$$

onde $\mathbf{V}_{\text {apl }}$ é o esforço cortante atuante na seção transversal.

Assim, está-se admitindo que as armaduras transversais só serão solicitadas após a danificação de algum ponto ao longo da seção transversal. Tal hipótese é respaldada por resultados de ensaios experimentais onde se verifica que as deformações nos estribos passam a ser expressivas após o início do processo de fissuração do concreto.

Segundo este modelo, uma vez ultrapassado o limite elástico do aço, deve-se aumentar a taxa de armadura transversal.

\subsection{ABORDAGEM DO PROCESSO DE RESOLUÇÃO}

Considere-se a iteração $\mathbf{n}$ do processo incremental iterativo comentado no item 5.1. Para cada ponto do contorno e do domínio da laje, tem-se os seguintes passos de resolução do problema:

Passo 1: Para cada ponto ao longo da seção transversal da laje calcula-se o incremento de deformações elásticas, $\Delta \varepsilon_{\mathbf{n}}^{\mathrm{e}}$, devido ao carregamento, somando-o ao vetor das deformações verdadeiras da iteração anterior. Obtém-se, assim, o vetor das deformações totais $\boldsymbol{\varepsilon}_{\mathbf{n}}$.

Passo 2: Utilizando-se a lei de Hooke, calculam-se as tensões elásticas, $\boldsymbol{\sigma}_{\mathbf{n}}^{\mathbf{e}}$.

Passo 3: Através da eq.(4.84), para pontos pertencentes ao concreto, calculase o alongamento equivalente $\overline{\boldsymbol{\varepsilon}}_{\mathbf{n}}$; verifica-se, então, o critério:

- Se $\bar{\varepsilon}_{\mathbf{n}} \leq \boldsymbol{\varepsilon}_{\mathbf{d o}}$, o ponto não está danificado; passa-se ao próximo ponto de Gauss;

- Se $\overline{\boldsymbol{\varepsilon}}_{\mathbf{n}}>\boldsymbol{\varepsilon}_{\mathbf{d o}}$, o ponto está danificado. Compara-se $\overline{\boldsymbol{\varepsilon}}_{\mathbf{n}}$ com a deformação equivalente da iteração anterior $\mathbf{S}(\mathbf{D})=\overline{\boldsymbol{\varepsilon}}_{\mathbf{n - 1}}$;

- Se $\mathbf{f}=\overline{\boldsymbol{\varepsilon}}_{\mathbf{n}}-\overline{\boldsymbol{\varepsilon}}_{\mathbf{n}-\mathbf{1}}>\mathbf{0}$, tem-se o caso de carregamento e a variável de dano é atualizada através da eq.(4.89). O estado de tensão verdadeiro é dado pela eq.(4.78). 
- Se $\mathbf{f}=\overline{\boldsymbol{\varepsilon}}_{\mathbf{n}}-\overline{\boldsymbol{\varepsilon}}_{\mathbf{n - 1}} \leq \mathbf{0}$, tem-se o caso de descarregamento. Esta situação é possível dado o procedimento utilizado para anular o esforço normal. Neste caso não se atualiza a variável de dano, uma vez que o incremento é elástico.

- $\quad$ Passo 4: Para os pontos relativos à armadura, utilizando-se a lei de Hooke, determinam-se os incrementos de tensões e tensões totais. Adotando-se o procedimento descrito em OWEN \& HINTON (1980), obtém-se a tensão verdadeira $\boldsymbol{\sigma}_{\mathbf{i}}^{\mathbf{v}(\mathbf{n})}$ e o incremento de tensão verdadeiro $\Delta \sigma_{i}^{\mathbf{v}(\mathbf{n})}$. Com isso, fica determinada a nova distribuição de tensões ao longo da seção transversal.

Passo 5: Segundo o processo dado no item 5.3, verifica-se o esforço normal nas direções $\mathbf{x}_{1}, \mathbf{x}_{2}$ e $\mathbf{x}_{1} \mathbf{x}_{2}$. Estima-se a nova posição da L.N., obtendo-se o incremento de deformações devido a esta nova posição, segundo a eq. (5.13). calculam-se as deformações totais e verificam-se os modelos constitutivos em todos os pontos ao longo da seção transversal calculando-se, a seguir, os novos esforços normais. Ao se obterem esforços nulos nas três direções, tem-se obtida a real distribuição de tensões ao longo da seção transversal.

Passo 6: A partir da eq.(5.7.a) obtém-se o vetor de momentos verdadeiros $\left\{\mathbf{M}_{\mathbf{v e r}}\right\}$ e o vetor de incremento de momentos verdadeiros $\left\{\Delta \mathbf{M}_{\mathbf{v e r}}\right\}$ relativos ao incremento em curso. $\mathrm{O}$ vetor de momentos residuais, a ser reaplicado na próxima iteração é determinado por:

$$
\left\{\Delta M^{0}\right\}^{n+1}=\left\{\Delta M^{e}\right\}^{\mathbf{n}}-\left\{\Delta M_{\text {ver }}\right\}^{n}
$$

Passo 7: Verifica-se o critério de convergência dado no item 5.1. Se tal critério não for verificado, aplica-se o vetor de momentos residuais dado na eq.(5.19) na forma de um campo de momentos iniciais, utilizando-se a eq.(5.2.b).

Passo 8: Calcula-se o esforço cortante no concreto, através da eq. (5.17), e o cortante na armadura transversal, através da eq. (5.18). 
Ao final de cada incremento, ter-se-ão obtidos os esforços, rotações e deslocamentos nos pontos do domínio e do contorno.

Passo 9: Passa-se ao incremento seguinte, repetindo-se todo o processo.

O fluxograma do programa principal encontra-se no apêndice D. Maiores detalhes quanto ao programa desenvolvido podem ser obtidos junto à biblioteca do Departamento de Estruturas da Escola de Engenharia de São Carlos, Universidade de São Paulo.

\subsection{APLICAÇÕES}

\subsubsection{ANÁLISE LINEAR}

\section{a. Exemplo 1.}

Analisa-se, neste exemplo, uma placa retangular de dimensões $300 \mathrm{~cm}$ x $600 \mathrm{~cm}$ simplesmente apoiada nas bordas com a condição "Soft”, conforme ilustra a fig.5.6.

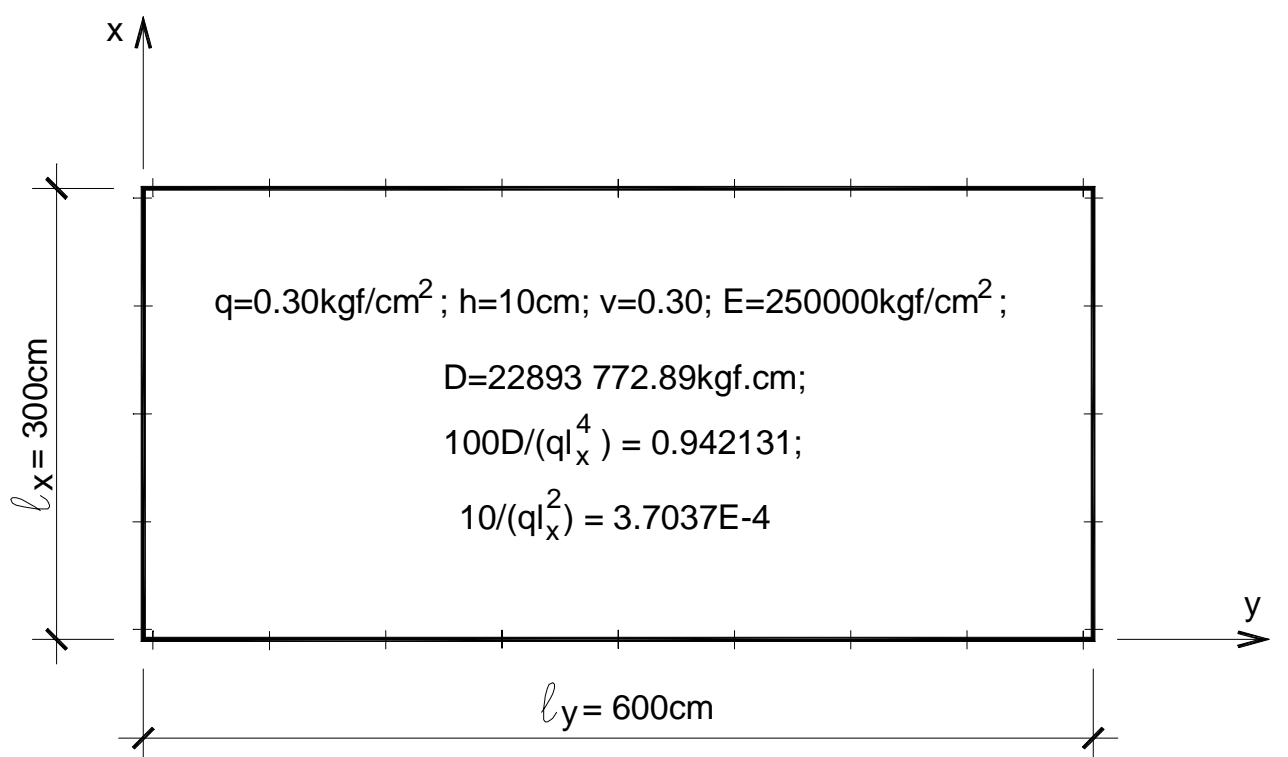

FIGURA 5.6. Placa retangular simplesmente apoiada nas bordas ( SOFT CONDITION) 
Neste caso, o contorno foi dividido em 12 elementos. Outros dados de interesse são dados na própria figura.

Os resultados obtidos para este exemplo são dados nas tabelas a seguir.

a.1. Deslocamento transversal $\mathrm{w}_{\mathrm{x}}$ sobre a linha de centro $\left(l_{\mathrm{y}} / 2\right)$.

\begin{tabular}{|c|c|c|c|c|}
\hline \multicolumn{5}{|c|}{$-\mathrm{w}_{\mathrm{x}}\left(100 \mathrm{D} /\left(\mathrm{q} l_{\mathrm{x}}^{4}\right)\right)$} \\
\hline & $0.125 l_{\mathrm{x}}$ & $0.25 l_{\mathrm{x}}$ & $0.375 l_{\mathrm{x}}$ & $0.50 l_{\mathrm{x}}$ \\
\hline PRESENTE ESTUDO & 0.4007 & 0.7332 & 0.9508 & 1.0263 \\
\hline TIMOSHENKO (1959) & & & & 1.0128 \\
\hline
\end{tabular}

a.2. Deslocamento transversal $\mathrm{w}_{\mathrm{x}}$ em $l_{\mathrm{y}} / 4$.

\begin{tabular}{|c|c|c|c|}
\hline \multicolumn{4}{|c|}{$-\mathrm{w}_{\mathrm{x}}\left(100 \mathrm{D} /\left(\mathrm{ql}_{\mathrm{x}}{ }^{4}\right)\right)$} \\
\hline $0.125 l_{\mathrm{x}}$ & $0.25 l_{\mathrm{x}}$ & $0.375 l_{\mathrm{x}}$ & $0.50 l_{\mathrm{x}}$ \\
\hline 0.3013 & 0.5363 & 0.7342 & 0.7919 \\
\hline
\end{tabular}

a.3. Deslocamento transversal $\mathrm{w}_{\mathrm{y}}-$ linha de centro $\left(l_{\mathrm{x}} / 2\right)$.

\begin{tabular}{|r|c|c|c|c|c|c|}
\hline & $-\mathrm{w}_{\mathrm{x}}\left(100 \mathrm{D} /\left(\mathrm{q}_{\mathrm{x}}{ }^{4}\right)\right)$ \\
\hline & $0.083 l_{\mathrm{y}}$ & $0.166 l_{\mathrm{y}}$ & $0.249 l_{\mathrm{y}}$ & $0.332 l_{\mathrm{y}}$ & $0.415 l_{\mathrm{y}}$ & $0.50 l_{\mathrm{y}}$ \\
\hline PRESENTE ESTUDO & 0.3234 & 0.5926 & 0.7919 & 0.9257 & 1.0017 & 1.0263 \\
\hline TIMOSHENKO (1959) & & & & & & 1.0128 \\
\hline
\end{tabular}

a.4. Momento fletor $\mathrm{M}_{\mathrm{xx}}$ sobre a linha de centro $\left(l_{\mathrm{y}} / 2\right)$.

\begin{tabular}{|c|c|c|c|c|}
\hline & $-\mathrm{M}_{\mathrm{xx}}\left(10 /\left(\mathrm{q} l_{\mathrm{x}}^{2}\right)\right)$ \\
\hline & $0.125 l_{\mathrm{x}}$ & $0.25 l_{\mathrm{x}}$ & $0.375 l_{\mathrm{x}}$ & $0.50 l_{\mathrm{x}}$ \\
\hline PRESENTE ESTUDO & 0.4654 & 0.7811 & 0.9650 & 1.025 \\
\hline TIMOSHENKO (1959) & & & & 1.017 \\
\hline
\end{tabular}

a.5. Momento fletor $\mathrm{M}_{\mathrm{xx}}$ em $l_{\mathrm{y}} / 4$.

\begin{tabular}{|c|c|c|c|}
\hline \multicolumn{5}{|c|}{$-\mathrm{M}_{\mathrm{xx}}\left(10 /\left(\mathrm{ql}_{\mathrm{x}}{ }^{2}\right)\right)$} \\
\hline $0.125 l_{\mathrm{x}}$ & $0.25 l_{\mathrm{x}}$ & $0.375 l_{\mathrm{x}}$ & $0.50 l_{\mathrm{x}}$ \\
\hline 0.3820 & 0.6305 & 0.7696 & 0.8143 \\
\hline
\end{tabular}

a.6. Momento fletor $\mathrm{M}_{\mathrm{yy}}$ sobre a linha de centro $l_{\mathrm{x}} / 2$.

\begin{tabular}{|c|c|c|c|c|c|c|}
\hline \multicolumn{7}{|c|}{$-\mathrm{M}_{\mathrm{yy}}\left(10 /\left(\mathrm{ql}_{\mathrm{x}}^{2}\right)\right)$} \\
\hline & $0.083 l_{\mathrm{y}}$ & $0.166 l_{\mathrm{y}}$ & $0.249 l_{\mathrm{y}}$ & $0.332 l_{\mathrm{y}}$ & $0.415 l_{\mathrm{y}}$ & $0.50 l_{\mathrm{y}}$ \\
\hline PRESENTE ESTUDO & 0.2955 & 0.4182 & 0.4600 & 0.4697 & 0.4685 & 0.4670 \\
\hline TIMOSHENKO (1959) & & & & & & 0.4640 \\
\hline
\end{tabular}




\section{b. Exemplo 2.}

Analisa-se aqui, a mesma placa do exemplo 1, agora com uma viga interna de seção transversal $10 \mathrm{~cm}$ x $30 \mathrm{~cm}$ na posição $l_{\mathrm{y}} / 2$, dividida em 4 barras, como mostra a fig.5.7.

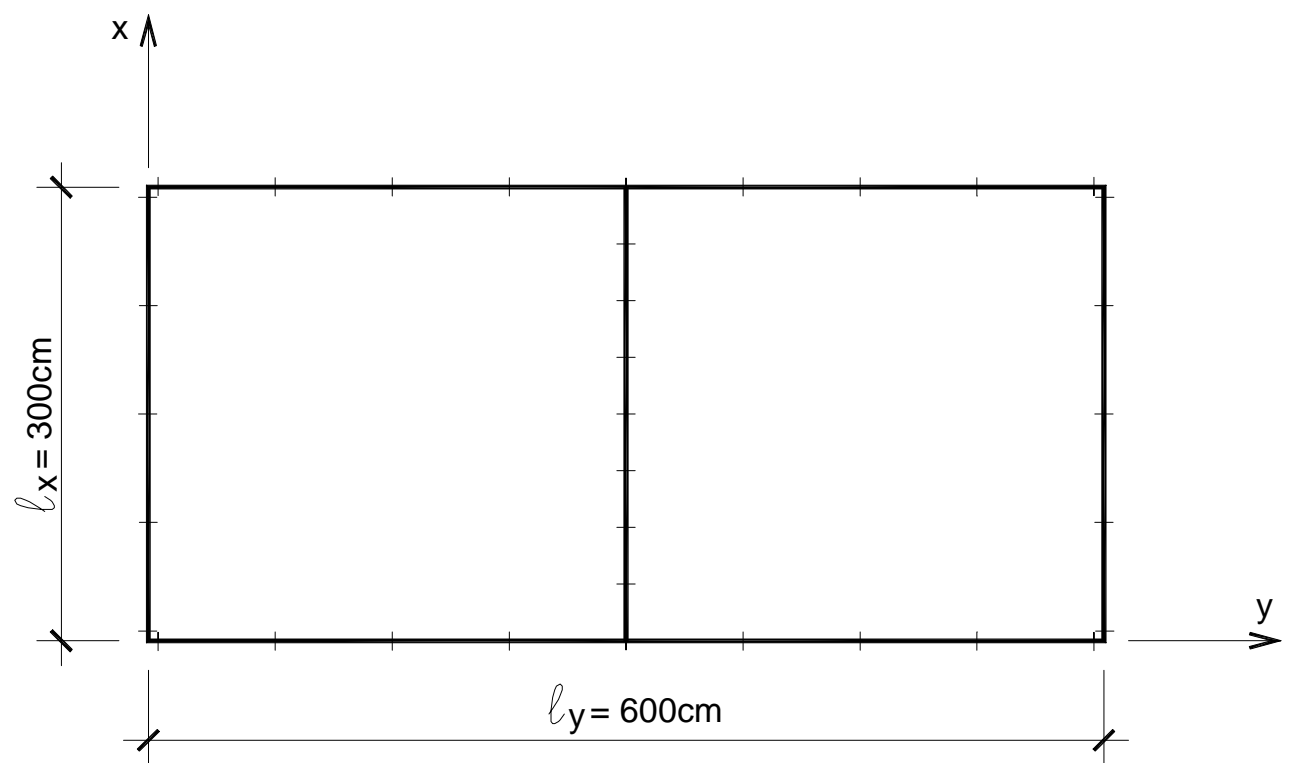

FIGURA 5.7. Placa retangular com viga interna

Nesta condição, os resultados obtidos foram:

b.1. Deslocamento transversal $\mathrm{w}_{\mathrm{x}}$ sobre a linha de centro $l_{\mathrm{y}} / 2$. (sobre a viga)

\begin{tabular}{|l|l|r|c|}
\hline \multicolumn{4}{|c|}{$-\mathrm{w}_{\mathrm{x}}\left(100 \mathrm{D} /\left(\mathrm{ql}_{\mathrm{x}}{ }^{4}\right)\right)$} \\
\hline $0.125 l_{\mathrm{x}}$ & $0.25 l_{\mathrm{x}}$ & $0.375 l_{\mathrm{x}}$ & $0.50 l_{\mathrm{x}}$ \\
\hline 0.058 & 0.1149 & 0.1564 & 0.1715 \\
\hline
\end{tabular}

b.2. Deslocamento transversal $\mathrm{w}_{\mathrm{x}}$ sobre a linha de centro $l_{\mathrm{y}} / 4$.

\begin{tabular}{|c|c|c|c|c|}
\hline \multicolumn{5}{|c|}{$-\mathrm{w}_{\mathrm{x}}\left(100 \mathrm{D} /\left(\mathrm{ql}_{\mathrm{x}}^{4}\right)\right)$} \\
\hline & $0.125 l_{\mathrm{x}}$ & $0.25 l_{\mathrm{x}}$ & $0.375 l_{\mathrm{x}}$ & $0.50 l_{\mathrm{x}}$ \\
\hline PRESENTE ESTUDO & 0.1465 & 0.2646 & 0.3398 & 0.3654 \\
\hline TIMOSHENKO (1959) (*) & & & & 0.2800 \\
\hline
\end{tabular}


b.3. Deslocamento transversal $\mathrm{w}_{\mathrm{y}}-$ linha de centro $l_{\mathrm{x}} / 2$.

\begin{tabular}{|r|c|c|c|c|c|c|}
\hline \multicolumn{1}{|c}{$-\mathrm{w}_{\mathrm{x}}\left(100 \mathrm{D} /\left(\mathrm{ql}_{\mathrm{x}}{ }^{4}\right)\right)$} \\
\hline & $0.083 l_{\mathrm{y}}$ & $0.166 l_{\mathrm{y}}$ & $0.249 l_{\mathrm{y}}$ & $0.332 l_{\mathrm{y}}$ & $0.415 l_{\mathrm{y}}$ & $0.50 l_{\mathrm{y}}$ \\
\hline PIMOSHENTE ESTUDO & 0.1952 & 0.3246 & 0.3654 & 0.3234 & 0.2323 & 0.1715 \\
\hline TIMOSHO (1959) (*) & & & 0.2800 & & & \\
\hline
\end{tabular}

b.4. Momento fletor $\mathrm{M}_{\mathrm{xx}}$ sobre a linha de centro $l_{\mathrm{y}} / 2$.

\begin{tabular}{|c|c|c|c|}
\hline \multicolumn{4}{|c|}{$-\mathrm{M}_{\mathrm{xx}}\left(10 /\left(\mathrm{ql}_{\mathrm{x}}{ }^{2}\right)\right)$} \\
\hline $0.125 l_{\mathrm{x}}$ & $0.25 l_{\mathrm{x}}$ & $0.375 l_{\mathrm{x}}$ & $0.50 l_{\mathrm{x}}$ \\
\hline-0.0777 & -0.0553 & 0.0207 & 0.0098 \\
\hline
\end{tabular}

b.5. Momento fletor $\mathrm{M}_{\mathrm{xx}}$ sobre a linha de centro $l_{\mathrm{y}} / 4$.

\begin{tabular}{|c|c|c|c|c|}
\hline & $\mathrm{M}_{\mathrm{xx}}\left(10 /\left(\mathrm{ql}_{\mathrm{x}}{ }^{2}\right)\right)$ \\
\hline & $0.125 l_{\mathrm{x}}$ & $0.25 l_{\mathrm{x}}$ & $0.375 l_{\mathrm{x}}$ & $0.50 l_{\mathrm{x}}$ \\
\hline PIMOSHENKO $(1959)(*)$ & & & & 0.3400 \\
\hline
\end{tabular}

b.6. Momento fletor na viga

Em kN.m

\begin{tabular}{|r|c|c|c|c|}
\hline & $0.125 l$ & $0.25 l$ & $0.375 l$ & $0.50 l$ \\
\hline PRESENTE ESTUDO & -19.8 & -23.1 & -32.8 & -42.5 \\
\hline VALOR APROXIMADO (**) & & & & -50.6 \\
\hline
\end{tabular}

b.7. Momento fletor $\mathrm{M}_{\mathrm{yy}}$ sobre a linha de centro $l_{\mathrm{x}} / 2$.

\begin{tabular}{|c|c|c|c|c|c|c|}
\hline & $0.083 l_{\mathrm{y}}$ & $0.166 l_{\mathrm{y}}$ & $0.249 l_{\mathrm{y}}$ & $0.332 l_{\mathrm{y}}$ & $0.415 l_{\mathrm{y}}$ & $0.50 l_{\mathrm{y}}$ \\
\hline PRESENTE ESTUDO & 0.2990 & 0.4118 & 0.4031 & 0.2776 & -0.0188 & -0.5618 \\
\hline $\begin{array}{c}\text { TIMOSHENKO } \\
(1959)(*)\end{array}$ & & & 0.3900 & & & -0.8400 \\
\hline
\end{tabular}

(*) tomou-se como referência os valores máximos relativos a uma placa de dimensões $300 \mathrm{~cm} \times 300 \mathrm{~cm}$ simplesmente apoiada em três lados e engastada no outro.

(**) Carregamento sobre a viga estimado pela teoria das charneiras plásticas (linhas de ruptura).

Os exemplos 1 e 2 permitem-nos concluir, como era de se esperar, que a placa com a viga interna (exemplo 2) tornou-se mais rígida. $\mathrm{O}$ deslocamento relativo no centro da placa (em $l_{\mathrm{y}} / 2$ ), para o exemplo 1 foi de 1.0263. No mesmo ponto, porém, no exemplo 2, o deslocamento verificado foi de 0.1715 .

Para o exemplo 2 observou-se que o deslocamento relativo em $l_{\mathrm{y}} / 4$, no centro da linha, foi maior do que o verificado em $l_{\mathrm{y}} / 2$ (0.3654 contra 0.1715$)$, o que está absolutamente coerente. 
Quanto aos momentos fletores $\mathbf{M}_{\mathbf{y y}}$, observa-se que, com a presença da viga interna, houve mudança de sinal entre os pontos $0.332 l_{\mathrm{y}}$ e $0.415 l_{\mathrm{y}}$ (ver tab. item b.7).

\section{c. Exemplo 3.}

Esta análise foi realizada considerando-se uma placa quadrada de dimensões $600 \mathrm{~cm}$ x $600 \mathrm{~cm}$ engastada nos quatro lados. Os dados adicionais encontram-se na fig.5.8. Neste caso, o contorno foi dividido em 16 elementos.

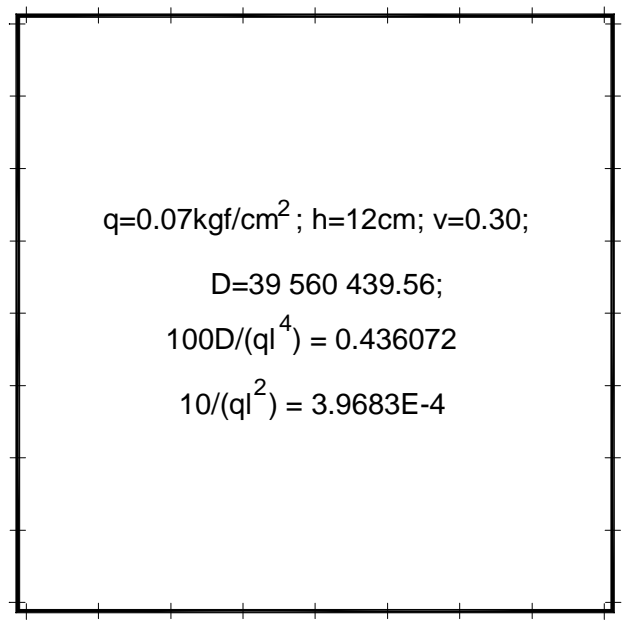

FIGURA 5.8. Placa quadrada engastada nos quatro lados

Os resultados dessa análise foram:

c.1. Deslocamento transversal w sobre a linha de centro

\begin{tabular}{|c|c|c|c|}
\hline$-\mathrm{w}\left(100 \mathrm{D} /\left(\mathrm{q} l^{4}\right)\right)$ \\
\hline & $0.1667 l$ & $0.3333 l$ & $0.50 l$ \\
\hline PRESENTE ESTUDO & 0.0442 & 0.1038 & 0.1276 \\
\hline TIMOSHENKO $(1959)$ & & & 0.1260 \\
\hline
\end{tabular}

c.2. Momento fletor sobre a linha de centro

\begin{tabular}{|c|c|c|c|}
\hline$-\mathrm{M}\left(10 /\left(\mathrm{q} l^{2}\right)\right)$ \\
\hline & $0.1667 l$ & $0.3333 l$ & $0.50 l$ \\
\hline PRESENTE ESTUDO & -0.0132 & 0.1812 & 0.2294 \\
\hline TIMOSHENKO $(1959)$ & & & 0.2310 \\
\hline
\end{tabular}




\section{d. Exemplo 4.}

Analisa-se, agora, a mesma placa do exemplo anterior, porém, apoiada sobre quatro vigas elásticas com seção transversal de $10 \mathrm{~cm}$ x $50 \mathrm{~cm}$ com quatro apoios rígidos nos cantos, como ilustra a fig.5.9.

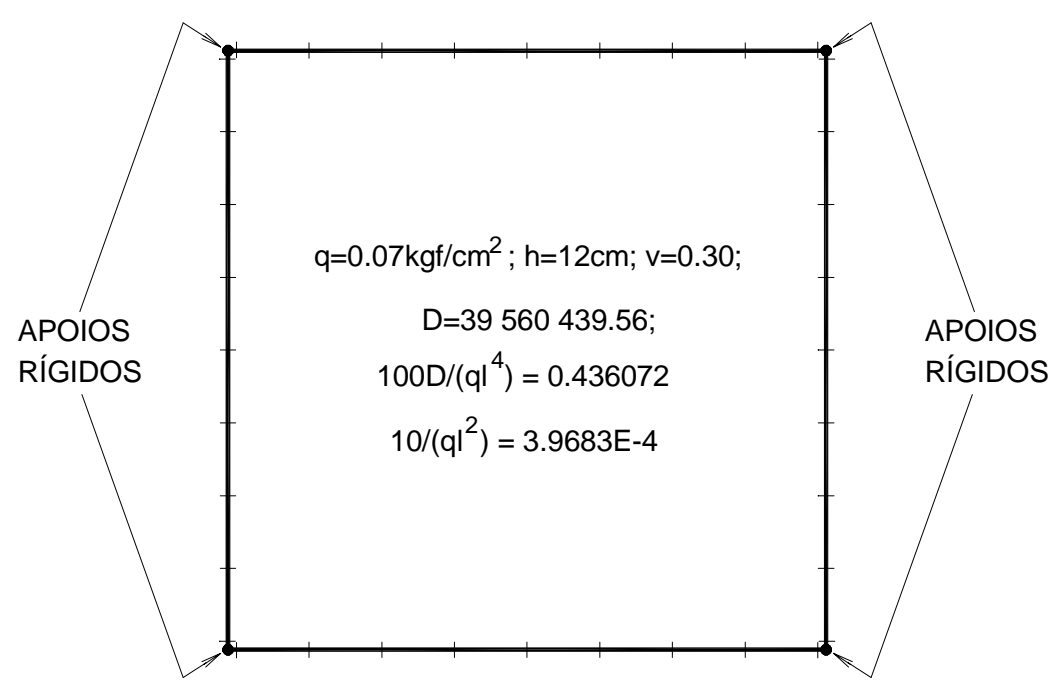

FIGURA 5.9. Placa quadrada sobre quatro vigas elásticas

Como resultado, foram obtidos os seguintes valores:

d.1. Deslocamento transversal w sobre a linha de centro

\begin{tabular}{|c|c|c|c|}
\hline$-\mathrm{w}\left(100 \mathrm{D} /\left(\mathrm{q} l^{4}\right)\right)$ & \\
\hline & $0.1667 l$ & $0.3333 l$ & $0.50 l$ \\
\hline PRESENTE ESTUDO & 0.6150 & 0.7983 & 0.8649 \\
\hline TIMOSHENKO (1959) $(*)$ & & & 0.4060 \\
\hline
\end{tabular}

d.2. Momento fletor sobre a linha de centro

\begin{tabular}{|c|c|c|c|}
\hline \multicolumn{4}{|c|}{$-\mathrm{M}\left(10 /\left(\mathrm{q} l^{2}\right)\right)$} \\
\hline & $0.1667 l$ & $0.3333 l$ & $0.50 l$ \\
\hline PRESENTE ESTUDO & -0.0074 & 0.1877 & 0.2492 \\
\hline TIMOSHENKO (1959) (*) & & & 0.4790 \\
\hline
\end{tabular}

(*) Estes resultados foram obtidos para uma placa simplesmente apoiada nos quatro lados. 
Dos exemplos 3 e 4, pode-se observar que a placa apoiada sobre quatro vigas elásticas (exemplo 4) tornou-se mais flexível em relação àquela engastada dos quatro lados (exemplo 3). O deslocamento relativo observado no primeiro caso foi de 0.8649 no centro da placa, contra o valor de 0.1276 para a placa engastada.

Apesar do fato de se estar comparando placas com condições de contorno diferentes no exemplo 4 (presente estudo: placa apoiada sobre vigas elásticas; TIMOSHENKO(1959), placa simplesmente apoiada nos quatro lados), os resultados mostram-se plenamente compatíveis. Senão vejamos: no presente estudo obteve-se um deslocamento relativo de 0.8649, superior a 0.4060 de TIMOSHENKO(1959). Em contrapartida, o momento fletor no presente estudo mostrou-se inferior àquele verificado por TIMOSHENKO (1959). Absolutamente coerente, portanto.

\subsubsection{ANÁLISE NÃO LINEAR}

\section{a. Exemplo 1}

Considere-se a laje esquematizada na fig. (5.10). Este exemplo foi analisado por NEVES (2000) via Método dos Elementos Finitos. Trata-se de uma análise não linear, utilizando-se a teoria de vigas de Timoshenko. Considera, portanto, as

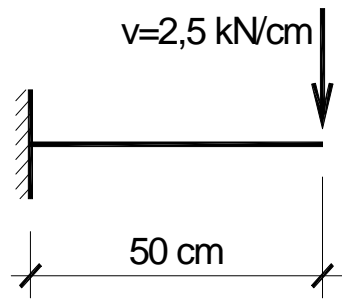

a) esquema estático

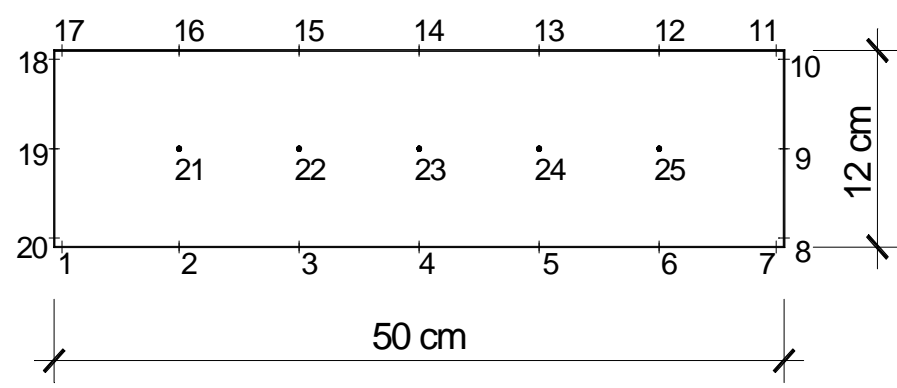

b) discretização do contorno

\section{FIGURA 5.10. Viga em balanço}

deformações devidas ao cisalhamento. Os resultados dos exemplos obtidos no referido trabalho se mostraram bem próximos daqueles verificados experimentalmente. 
Dados: Módulo de elasticidade longitudinal do concreto: $2500 \mathrm{kN} / \mathrm{cm}^{2}$; coeficiente de Poisson: 0,25 ; tensão de escoamento do aço: $50 \mathrm{kN} / \mathrm{cm}^{2}$; Módulo de elasticidade longitudinal do aço: $20000 \mathrm{kN} / \mathrm{cm}^{2}$; parâmetro de endurecimento do aço: $2222,22 \mathrm{kN} / \mathrm{cm}^{2}$; armadura longitudinal: 2 camadas a $12,5 \mathrm{~cm}$ e $-12,5 \mathrm{~cm}$ da superfície média, com área de seção transversal de $2,5 \mathrm{~cm}^{2}$ cada; número de elementos de contorno: 8; número de incrementos:10; número máximo de iterações: 600; tolerâncias: 0,01\%; número de pontos de Gauss:12; altura da seção transversal: $30 \mathrm{~cm}$.

$\mathrm{Na}$ fig.5.11. apresentam-se as curvas cargas-deslocamentos para os pontos internos.

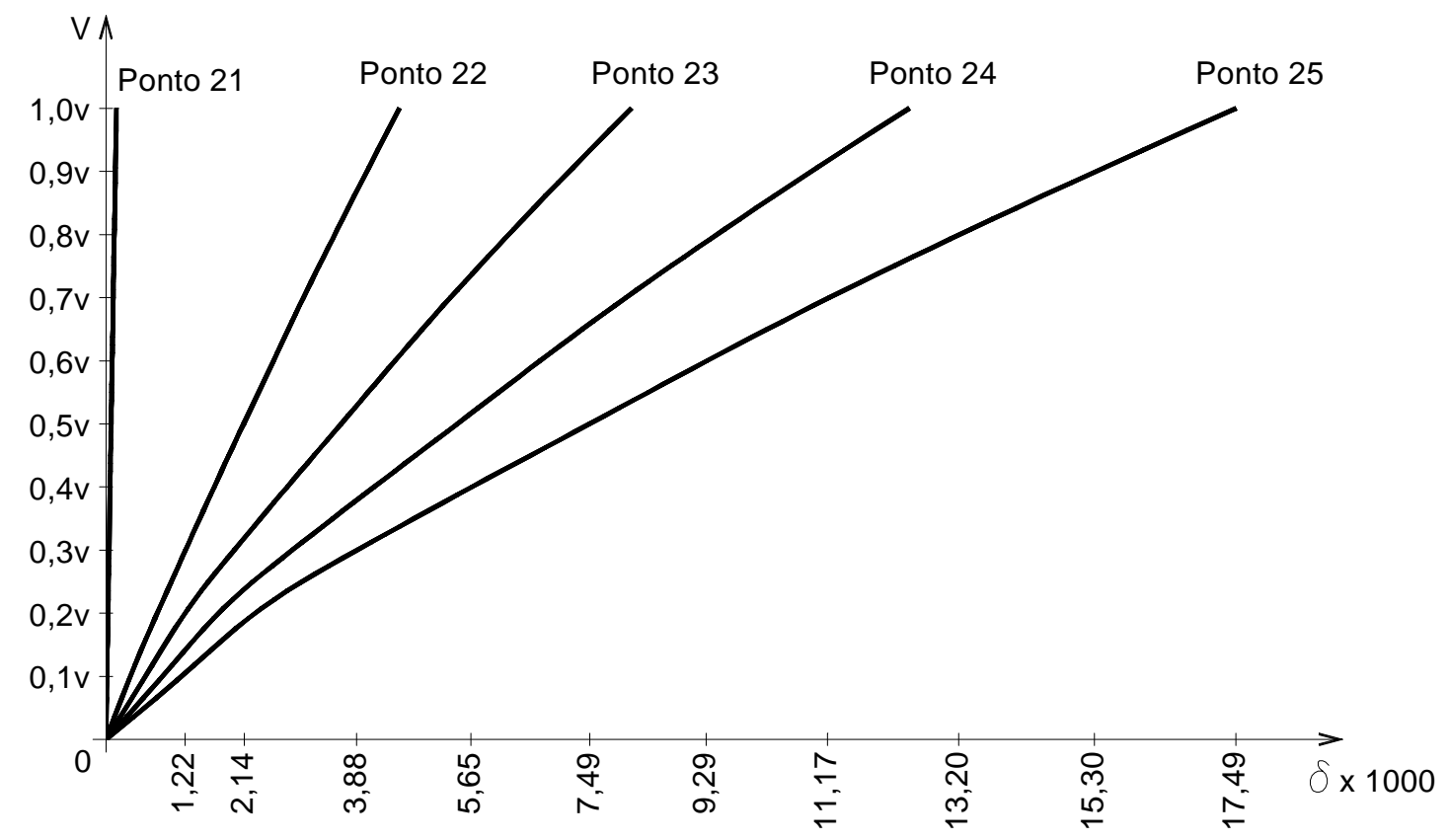

FIGURA 5.11. Curvas cargas-deslocamentos para os pontos internos

O deslocamento final para o ponto 9 obtido no presente trabalho foi de $0,02278 \mathrm{~cm}$. NEVES (2000) obteve o valor de $0,02785 \mathrm{~cm}$. Utilizando-se o programa computacional desenvolvido por FERNANDES (1998), encontrou-se o valor de 0,01591cm. Ali utilizou-se a teoria clássica de Kirchhoff. Como pode ser observado, a não consideração das deformações provocadas pelo cisalhamento, no 
exemplo em questão, fornece resultado bem inferior, quando comparado com o resultado obtido por NEVES (2000). O resultado obtido no presente estudo mostrase bastante satisfatório. 


\section{b. Exemplo 2}

Analisa-se a seguir uma laje quadrada de vão $l=400 \mathrm{~cm}$ e altura $h=7 \mathrm{~cm} \mathrm{e}$ altura útil $d=6,1 \mathrm{~cm}$, simplesmente apoiada. CAMPOS (2000) realizou diversos ensaios com lajes de mesma dimensão variando, ora a taxa de armadura longitudinal, ora a resistência do concreto, ensaiadas até um valor pré determinado de carregamento uniformemente distribuído. Naquele trabalho, tinha-se como objetivo impor às lajes um carregamento tal que a mesma atingisse um estado limite de utilização sem, todavia, chegar à ruptura, reforçando-as, posteriormente. Como referência, apenas uma laje foi ensaiada até a ruptura.

A primeira laje apresentou os seguintes dados: armadura longitudinal: $\Phi=$ $5 \mathrm{~mm}$ a cada $20 \mathrm{~cm}$ nas duas direções; os diagramas tensão-deformação do aço obtido experimentalmente e o adotado no presente estudo estão representados na fig.5.12; Módulo de elasticidade: $20.530 \mathrm{kN} / \mathrm{cm}^{2}$; parâmetro de endurecimento adotado no presente estudo: $2.000 \mathrm{kN} / \mathrm{cm}^{2}$; tensão inicial de escoamento adotada: $70 \mathrm{kN} / \mathrm{cm}^{2}$; concreto: resistência característica no dia do ensaio: $\mathrm{f}_{\mathrm{c}}=20,7 \mathrm{MPa}$; módulo de elasticidade: $1.730 \mathrm{kN} / \mathrm{cm}^{2}$; coeficiente de Poisson: 0,25 (adotado). O carregamento total aplicado foi de $6,50 \mathrm{kN} / \mathrm{m}^{2}$, desconsiderando-se o peso próprio da laje.

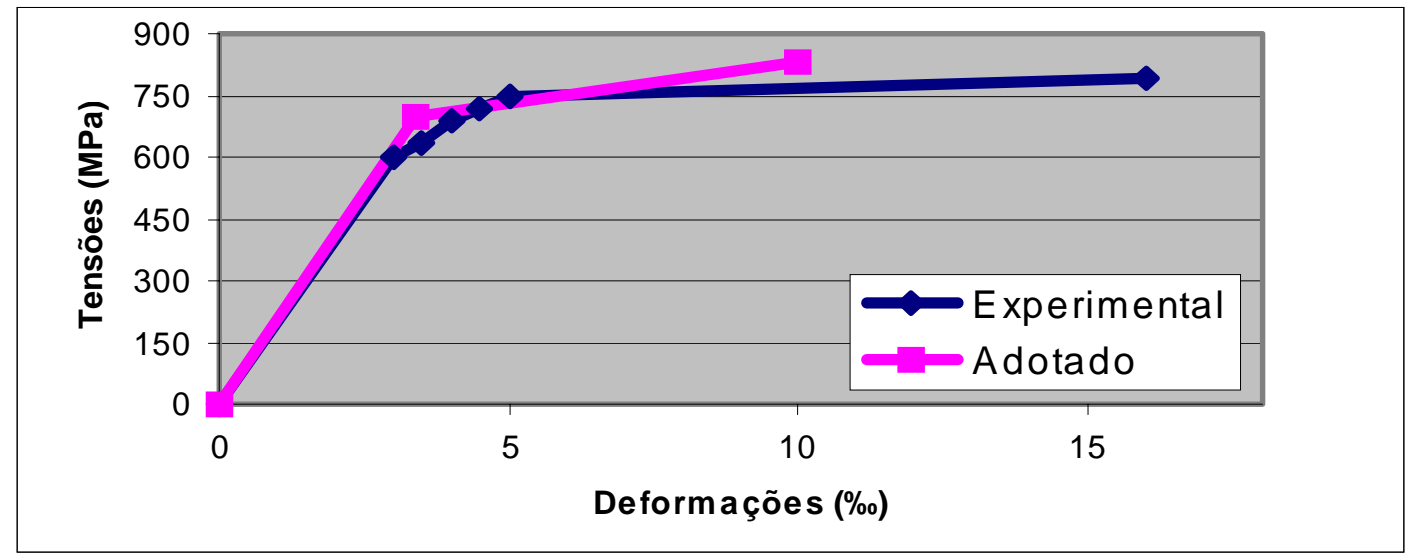

FIGURA 5.12. Curvas tensão - Deformação para o aço

No presente estudo, o contorno da laje foi discretizado em 8 elementos e o domínio em 88, 120 e 144 células. Tolerância de convergência do processo iterativo: 
$0,01 \%$. Os resultados obtidos para a flecha no centro da laje são dados na tabela a seguir.

\begin{tabular}{|c|c|c|c|c|}
\hline \multicolumn{5}{|c|}{ Flechas (mm) } \\
\hline $\operatorname{Carga}\left(\mathrm{kN} / \mathrm{m}^{2}\right)$ & Experimental & 88 células & 120 células & 144 células \\
\hline $\begin{array}{c}0.00 \\
\end{array}$ & 0.00 & 0.00 & 0.00 & $\mathbf{0 . 0 0}$ \\
\hline 0.25 & 0.71 & 0.61 & 0.60 & 0.60 \\
\hline 0.50 & 1.35 & 1.22 & 1.21 & 1.20 \\
\hline 0.75 & 1.93 & 1.85 & 1.88 & 1.87 \\
\hline 1.00 & 2.63 & 2.55 & 2.66 & 2.66 \\
\hline 1.25 & 3.35 & 3.32 & 3.58 & 3.63 \\
\hline 1.50 & 3.99 & 4.15 & 4.66 & 4.79 \\
\hline 1.75 & 4.82 & 5.07 & 6.00 & 6.18 \\
\hline 2.00 & 5.82 & 6.03 & 7.54 & 8.04 \\
\hline 2.25 & 8.20 & 7.09 & 9.50 & 10.54 \\
\hline 2.50 & 20.85 & 8.43 & 11.59 & 14.77 \\
\hline 2.75 & 24.96 & 10.03 & 14.91 & 19.83 \\
\hline 3.00 & 31.35 & 11.42 & 17.12 & 22.33 \\
\hline 3.50 & 40.85 & 14.64 & 21.97 & 31.84 \\
\hline 4.00 & 45.99 & 20.68 & 31.58 & 44.43 \\
\hline 4.50 & 51.57 & 26.30 & 36.78 & 55.46 \\
\hline 5.00 & 57.24 & 28.88 & 41.43 & 65.24 \\
\hline 5.50 & 63.16 & $\mathbf{3 4 . 4 7}$ & 50.23 & 76.39 \\
\hline 6.00 & 70.57 & 39.40 & 56.46 & 88.34 \\
\hline 6.25 & 73.73 & 41.48 & 59.23 & 96.08 \\
\hline 6.50 & 75.05 & 44.34 & 63.39 & 103.32 \\
\hline
\end{tabular}

As curvas carga - deslocamentos estão representadas na fig.5.13. 


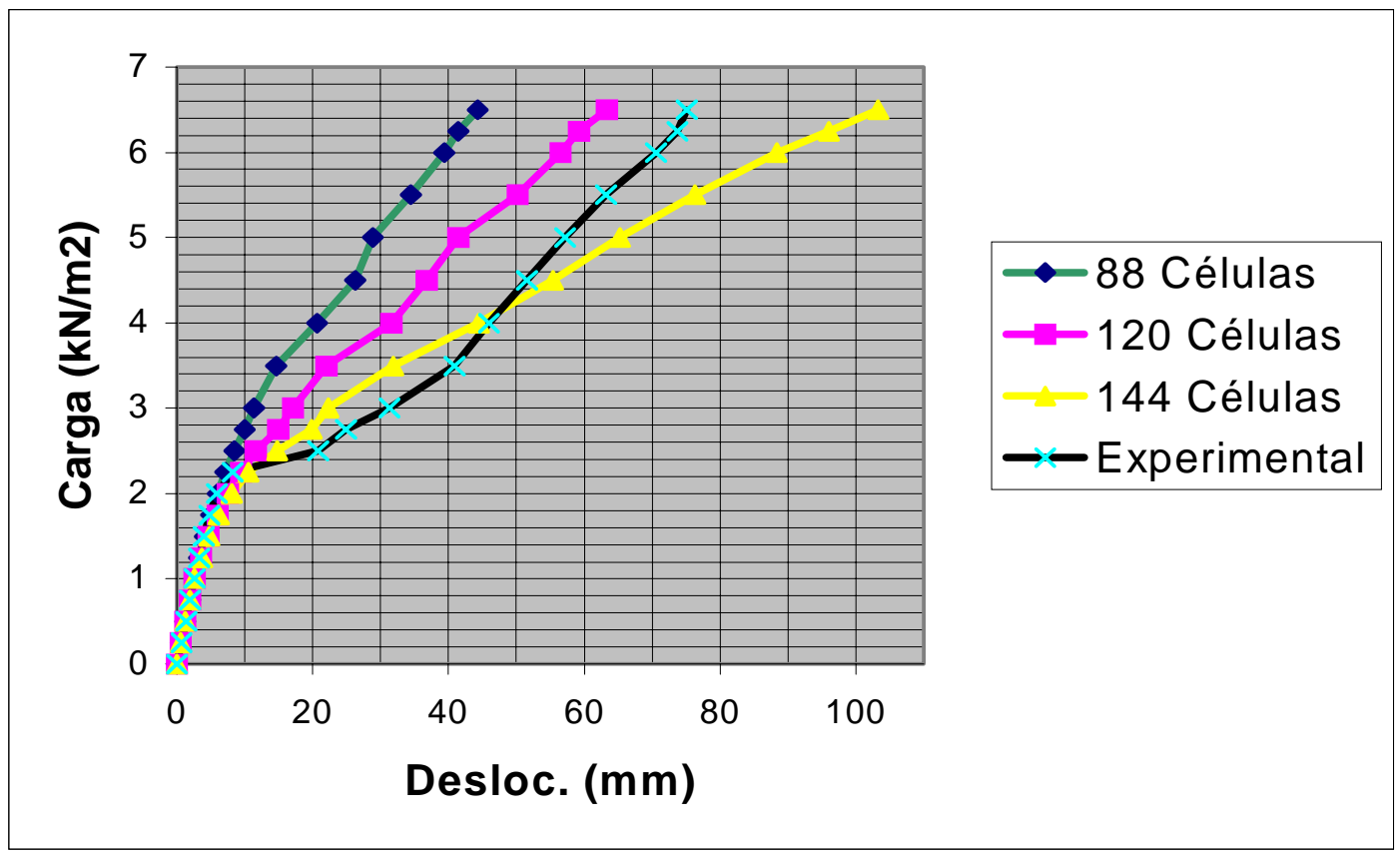

FIGURA 5.13. Curvas Cargas - Deslocamentos 1.

Como pode ser observado, até o carregamento de $2,0 \mathrm{kN} / \mathrm{m}^{2}$, o comportamento do material é próximo do elástico linear, ficando os resultados obtidos com 88, 120 e 144 células mais próximos dos resultados experimentais. À medida que o carregamento aumenta, intensifica-se a formação de fissuras na laje, havendo maiores acréscimos nos deslocamentos. Veja-se, por exemplo, que entre os carregamentos de $2,25 \mathrm{kN} / \mathrm{m}^{2}$ e $2,5 \mathrm{kN} / \mathrm{m}^{2}$, o deslocamento no centro da laje obtido experimentalmente passou de $8,2 \mathrm{~mm}$ para $20.85 \mathrm{~mm}$ ( $154 \%$ de acréscimo). Para a análise procedida com 144 células, o deslocamento no mesmo ponto passou de $10,54 \mathrm{~mm}$ para $14,77 \mathrm{~mm}$ (40\% de acréscimo).

Para o carregamento de serviço $\left(3,5 \mathrm{kN} / \mathrm{m}^{2}\right)$, observa-se uma discrepância de $28 \%$ entre os valores experimental $(40.85 \mathrm{~mm})$ e da análise com 144 células (31.84mm).

Para fins comparativos, CAMPOS (2000) ensaiou outra laje até a ruptura, com a mesma armadura e dimensões em planta e com altura útil $d=7,1 \mathrm{~cm}$. O concreto apresentou as seguintes características: $\mathbf{f}_{\mathbf{c}}=20,8 \mathrm{MPa}$, módulo de elasticidade $\mathbf{E}=1.606 \mathrm{kN} / \mathrm{cm}^{2}$. Altura total $\mathbf{h}=7,5 \mathrm{~cm}$ (adotado). Carregamento total aplicado: $9,25 \mathrm{kN} / \mathrm{m}^{2}$. 
Para a análise da laje, o contorno da mesma foi discretizado em 8 elementos e o domínio em 120 e 144 células. Os outros dados foram mantidos. Os resultados obtidos para a flecha no centro da laje são dados a seguir.

\begin{tabular}{|c|c|c|c|}
\hline \multicolumn{4}{|c|}{ Flechas (mm) } \\
\hline Carga $\left(\mathrm{kN} / \mathrm{m}^{2}\right)$ & Experimental & 120 Células & 144 Células \\
\hline 0.00 & 0.00 & 0.00 & 0.00 \\
\hline 0.50 & 0.84 & 1.06 & 1.05 \\
\hline 1.00 & 1.95 & 2.30 & 2.30 \\
\hline 1.50 & 3.15 & 3.99 & 4.08 \\
\hline 2.00 & 5.04 & 6.39 & 6.64 \\
\hline 2.50 & 29.39 & 9.76 & 10.67 \\
\hline 3.00 & 39.41 & 14.88 & 22.07 \\
\hline 3.50 & 44.98 & 21.47 & 34.41 \\
\hline 4.00 & 51.16 & 25.12 & 42.75 \\
\hline 4.50 & 58.56 & 33.22 & 48.50 \\
\hline 5.00 & 64.84 & 38.94 & 56.49 \\
\hline 5.50 & 70.34 & 46.14 & 67.67 \\
\hline 6.00 & 77.94 & 53.06 & 79.19 \\
\hline 6.50 & 91.81 & 57.12 & 90.26 \\
\hline 7.00 & 94.31 & 60.98 & 103.24 \\
\hline 7.50 & 99.11 & 66.04 & 117.79 \\
\hline 8.00 & 116.32 & 71.50 & 130.72 \\
\hline 8.50 & 121.14 & 78.83 & 143.18 \\
\hline 9.00 & 124.70 & 84.80 & 155.9 \\
\hline 9.25 & RUPTURA & - & \\
\hline 9.50 & - & 87.36 & 162.24 \\
\hline
\end{tabular}

As curvas cargas - deslocamentos para este caso estão representadas na fig. 5.14 . 


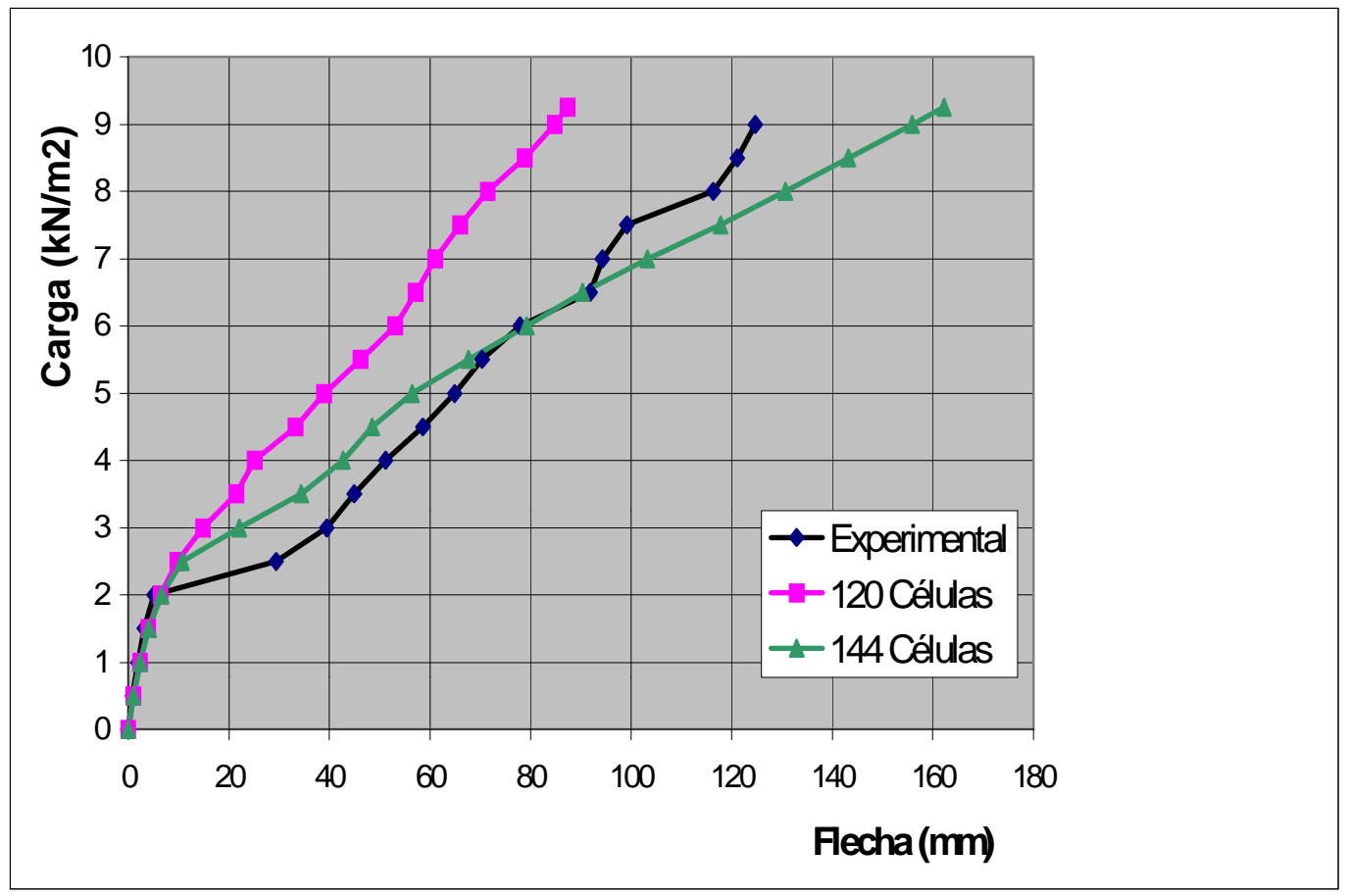

FIGURA 5.14. Curvas carga - deslocamentos 2.

Veja-se que, a partir do $5^{\circ}$ incremento de carga (carregamento de $2,5 \mathrm{kN} / \mathrm{m}^{2}$ ), os resultados numéricos que mais se aproximaram dos resultados experimentais foram aqueles relativos à maior discretização do domínio (144 células).

O comportamento da laje entre os carregamentos de $2,0 \mathrm{kN} / \mathrm{m}^{2}$ e $3,0 \mathrm{kN} / \mathrm{m}^{2}$, observado na curva experimental (fig. 5.14), demonstra uma considerável transferência de esforços de tração do concreto para o aço (fig. 5.16), devida ao processo de fissuração; após esta etapa inicial de formação de fissuras, houve uma “estabilização da rigidez”. O modelo de análise utilizado no presente estudo simula em parte este comportamento. Veja-se, considerando a discretização em 144 células (fig. 5.14), que a partir do carregamento de $2,0 \mathrm{kN} / \mathrm{m}^{2}$, há um decréscimo da rigidez, porém, de forma mais "suave" quando comparada à curva experimental.

Para o carregamento de $9,0 \mathrm{kN} / \mathrm{m}^{2}$, imediatamente anterior à ruptura da peça, o deslocamento relativo à análise com 144 células foi $19 \%$ superior ao deslocamento experimental.

Outros dados de interesse são aqueles relativos ao processo de danificação no concreto e às deformações nas armaduras durante a aplicação dos incrementos de 
carga. Para a verificação do dano, foram adotados 8 pontos de Gauss ao longo da espessura da laje, cujas ordenadas têm como referencial a linha média da seção transversal e sentido positivo para cima. Seus valores são:

\begin{tabular}{|c|c|c|c|c|c|c|c|}
\hline \multicolumn{7}{|c|}{ ORDENADAS DOS PONTOS DE GAUSS (cm) } \\
\hline \multicolumn{7}{|c|}{ PONTOS } \\
\hline $\mathbf{1}$ & 2 & 3 & 4 & 5 & 6 & 7 & 8 \\
\hline 0.6879 & $-\mathbf{- 0 . 6 8 7 9}$ & 1.9707 & -1.9707 & 2.9875 & -2.9875 & $\mathbf{3 . 6 0 1 1}$ & $\mathbf{- 3 . 6 0 1 1}$ \\
\hline
\end{tabular}

Os resultados para o ponto central, relativos à discretização do domínio em 144 células, estão tabelados a seguir e representados na fig. 5.15.

\begin{tabular}{|c|c|c|c|c|c|c|c|c|}
\hline \multicolumn{10}{|c|}{ Dano } \\
\hline $\begin{array}{c}\text { Carga } \\
\text { KN/ } \mathbf{m}^{2}\end{array}$ & $\mathbf{1}$ & $\mathbf{2}$ & $\mathbf{3}$ & $\mathbf{4}$ & $\mathbf{5}$ & $\mathbf{6}$ & $\mathbf{7}$ & $\mathbf{8}$ \\
\hline $\mathbf{0 . 0 0}$ & $\mathbf{0 . 0 0 0 0}$ & $\mathbf{0 . 0 0 0 0}$ & $\mathbf{0 . 0 0 0 0}$ & $\mathbf{0 . 0 0 0 0}$ & $\mathbf{0 . 0 0 0 0}$ & $\mathbf{0 . 0 0 0 0}$ & $\mathbf{0 . 0 0 0 0}$ & $\mathbf{0 . 0 0 0 0}$ \\
\hline $\mathbf{0 . 5 0}$ & $\mathbf{0 . 0 0 0 0}$ & $\mathbf{0 . 0 0 0 0}$ & $\mathbf{0 . 0 0 0 0}$ & $\mathbf{0 . 0 0 0 0}$ & $\mathbf{0 . 0 0 0 0}$ & $\mathbf{0 . 0 0 0 0}$ & $\mathbf{0 . 0 0 0 0}$ & $\mathbf{0 . 0 0 0 0}$ \\
\hline $\mathbf{1 . 0 0}$ & $\mathbf{0 . 0 0 0 0}$ & $\mathbf{0 . 0 0 0 0}$ & $\mathbf{0 . 0 0 0 0}$ & $\mathbf{0 . 0 0 0 0}$ & $\mathbf{0 . 0 0 0 0}$ & $\mathbf{0 . 0 7 7 2}$ & $\mathbf{0 . 0 0 0 0}$ & $\mathbf{0 . 1 8 6 1}$ \\
\hline $\mathbf{1 . 5 0}$ & $\mathbf{0 . 0 0 0 0}$ & $\mathbf{0 . 0 0 0 0}$ & $\mathbf{0 . 0 0 0 0}$ & $\mathbf{0 . 1 2 9 7}$ & $\mathbf{0 . 0 0 0 0}$ & $\mathbf{0 . 3 8 1 6}$ & $\mathbf{0 . 0 0 5 3}$ & $\mathbf{0 . 4 9 4 7}$ \\
\hline $\mathbf{2 . 0 0}$ & $\mathbf{0 . 0 0 0 0}$ & $\mathbf{0 . 1 8 1 6}$ & $\mathbf{0 . 0 0 0 0}$ & $\mathbf{0 . 5 7 8 8}$ & $\mathbf{0 . 0 2 8 7}$ & $\mathbf{0 . 7 4 4 4}$ & $\mathbf{0 . 0 8 9 6}$ & $\mathbf{0 . 8 0 8 2}$ \\
\hline $\mathbf{2 . 5 0}$ & $\mathbf{0 . 0 0 0 0}$ & $\mathbf{0 . 5 1 1 3}$ & $\mathbf{0 . 0 0 0 0}$ & $\mathbf{0 . 8 2 5 4}$ & $\mathbf{0 . 1 6 0 5}$ & $\mathbf{0 . 9 1 3 1}$ & $\mathbf{0 . 2 4 2 2}$ & $\mathbf{0 . 9 3 9 1}$ \\
\hline $\mathbf{3 . 0 0}$ & $\mathbf{0 . 7 6 0 4}$ & $\mathbf{0 . 9 5 2}$ & $\mathbf{0 . 0 8 7 5}$ & $\mathbf{0 . 9 8 0 7}$ & $\mathbf{0 . 2 7 1 1}$ & $\mathbf{0 . 9 8 7 8}$ & $\mathbf{0 . 3 7 2 0}$ & $\mathbf{0 . 9 9 0 5}$ \\
\hline $\mathbf{3 . 5 0}$ & $\mathbf{0 . 9 7 7 8}$ & $\mathbf{0 . 9 9 2 2}$ & $\mathbf{0 . 7 2 9 7}$ & $\mathbf{0 . 9 9 6 7}$ & $\mathbf{0 . 4 3 4 7}$ & $\mathbf{0 . 9 9 8 3}$ & $\mathbf{0 . 5 6 1 4}$ & $\mathbf{0 . 9 9 8 8}$ \\
\hline $\mathbf{4 . 0 0}$ & $\mathbf{0 . 9 7 7 8}$ & $\mathbf{0 . 9 9 2 3}$ & $\mathbf{0 . 7 2 9 7}$ & $\mathbf{0 . 9 9 7 4}$ & $\mathbf{0 . 4 3 4 7}$ & $\mathbf{0 . 9 9 8 9}$ & $\mathbf{0 . 5 6 1 4}$ & $\mathbf{0 . 9 9 9 2}$ \\
\hline $\mathbf{4 . 5 0}$ & $\mathbf{0 . 9 7 7 8}$ & $\mathbf{0 . 9 9 3 0}$ & $\mathbf{0 . 7 2 9 7}$ & $\mathbf{0 . 9 9 7 9}$ & $\mathbf{0 . 4 9 4 8}$ & $\mathbf{0 . 9 9 9 1}$ & $\mathbf{0 . 6 4 7 6}$ & $\mathbf{0 . 9 9 9 4}$ \\
\hline $\mathbf{5 . 0 0}$ & $\mathbf{0 . 9 7 7 8}$ & $\mathbf{0 . 9 9 3 9}$ & $\mathbf{0 . 7 2 9 7}$ & $\mathbf{0 . 9 9 8 5}$ & $\mathbf{0 . 6 3 6 6}$ & $\mathbf{0 . 9 9 9 4}$ & $\mathbf{0 . 7 4 9 8}$ & $\mathbf{0 . 9 9 9 6}$ \\
\hline $\mathbf{5 . 5 0}$ & $\mathbf{0 . 9 7 7 8}$ & $\mathbf{0 . 9 9 4 9}$ & $\mathbf{0 . 7 2 9 7}$ & $\mathbf{0 . 9 9 9 0}$ & $\mathbf{0 . 7 5 5 1}$ & $\mathbf{0 . 9 9 9 6}$ & $\mathbf{0 . 8 3 9 2}$ & $\mathbf{0 . 9 9 9 7}$ \\
\hline $\mathbf{6 . 0 0}$ & $\mathbf{0 . 9 7 7 8}$ & $\mathbf{0 . 9 9 5 6}$ & $\mathbf{0 . 7 2 9 7}$ & $\mathbf{0 . 9 9 9 3}$ & $\mathbf{0 . 8 2 3 5}$ & $\mathbf{0 . 9 9 9 7}$ & $\mathbf{0 . 8 9 0 2}$ & $\mathbf{0 . 9 9 9 8}$ \\
\hline $\mathbf{6 . 5 0}$ & $\mathbf{0 . 9 7 7 8}$ & $\mathbf{0 . 9 9 6 3}$ & $\mathbf{0 . 7 2 9 7}$ & $\mathbf{0 . 9 9 9 5}$ & $\mathbf{0 . 8 7 4 3}$ & $\mathbf{0 . 9 9 9 7}$ & $\mathbf{0 . 9 2 6 2}$ & $\mathbf{0 . 9 9 9 8}$ \\
\hline $\mathbf{7 . 0 0}$ & $\mathbf{0 . 9 7 7 8}$ & $\mathbf{0 . 9 9 6 8}$ & $\mathbf{0 . 7 5 2 3}$ & $\mathbf{0 . 9 9 9 6}$ & $\mathbf{0 . 9 1 1 6}$ & $\mathbf{0 . 9 9 9 8}$ & $\mathbf{0 . 9 5 1 2}$ & $\mathbf{0 . 9 9 9 8}$ \\
\hline $\mathbf{7 . 5 0}$ & $\mathbf{0 . 9 7 7 8}$ & $\mathbf{0 . 9 9 7 4}$ & $\mathbf{0 . 8 1 4 3}$ & $\mathbf{0 . 9 9 9 7}$ & $\mathbf{0 . 9 4 1 2}$ & $\mathbf{0 . 9 9 9 8}$ & $\mathbf{0 . 9 6 9 6}$ & $\mathbf{0 . 9 9 9 8}$ \\
\hline $\mathbf{8 . 0 0}$ & $\mathbf{0 . 9 7 7 8}$ & $\mathbf{0 . 9 9 7 8}$ & $\mathbf{0 . 8 5 7 0}$ & $\mathbf{0 . 9 9 9 7}$ & $\mathbf{0 . 9 5 9 4}$ & $\mathbf{0 . 9 9 9 8}$ & $\mathbf{0 . 9 8 0 1}$ & $\mathbf{0 . 9 9 9 9}$ \\
\hline $\mathbf{8 . 5 0}$ & $\mathbf{0 . 9 7 7 8}$ & $\mathbf{0 . 9 9 8 2}$ & $\mathbf{0 . 8 8 8 1}$ & $\mathbf{0 . 9 9 9 8}$ & $\mathbf{0 . 9 7 1 2}$ & $\mathbf{0 . 9 9 9 8}$ & $\mathbf{0 . 9 8 6 4}$ & $\mathbf{0 . 9 9 9 9}$ \\
\hline $\mathbf{9 . 0 0}$ & $\mathbf{0 . 9 7 7 8}$ & $\mathbf{0 . 9 9 8 4}$ & $\mathbf{0 . 9 1 1 9}$ & $\mathbf{0 . 9 9 9 8}$ & $\mathbf{0 . 9 7 9 3}$ & $\mathbf{0 . 9 9 9 9}$ & $\mathbf{0 . 9 9 0 5}$ & $\mathbf{0 . 9 9 9 9}$ \\
\hline $\mathbf{9 . 5 0}$ & $\mathbf{0 . 9 7 7 8}$ & $\mathbf{0 . 9 9 8 5}$ & $\mathbf{0 . 9 2 2 2}$ & $\mathbf{0 . 9 9 9 8}$ & $\mathbf{0 . 9 8 2 5}$ & $\mathbf{0 . 9 9 9 9}$ & $\mathbf{0 . 9 9 2 0}$ & $\mathbf{0 . 9 9 9 9}$ \\
\hline
\end{tabular}




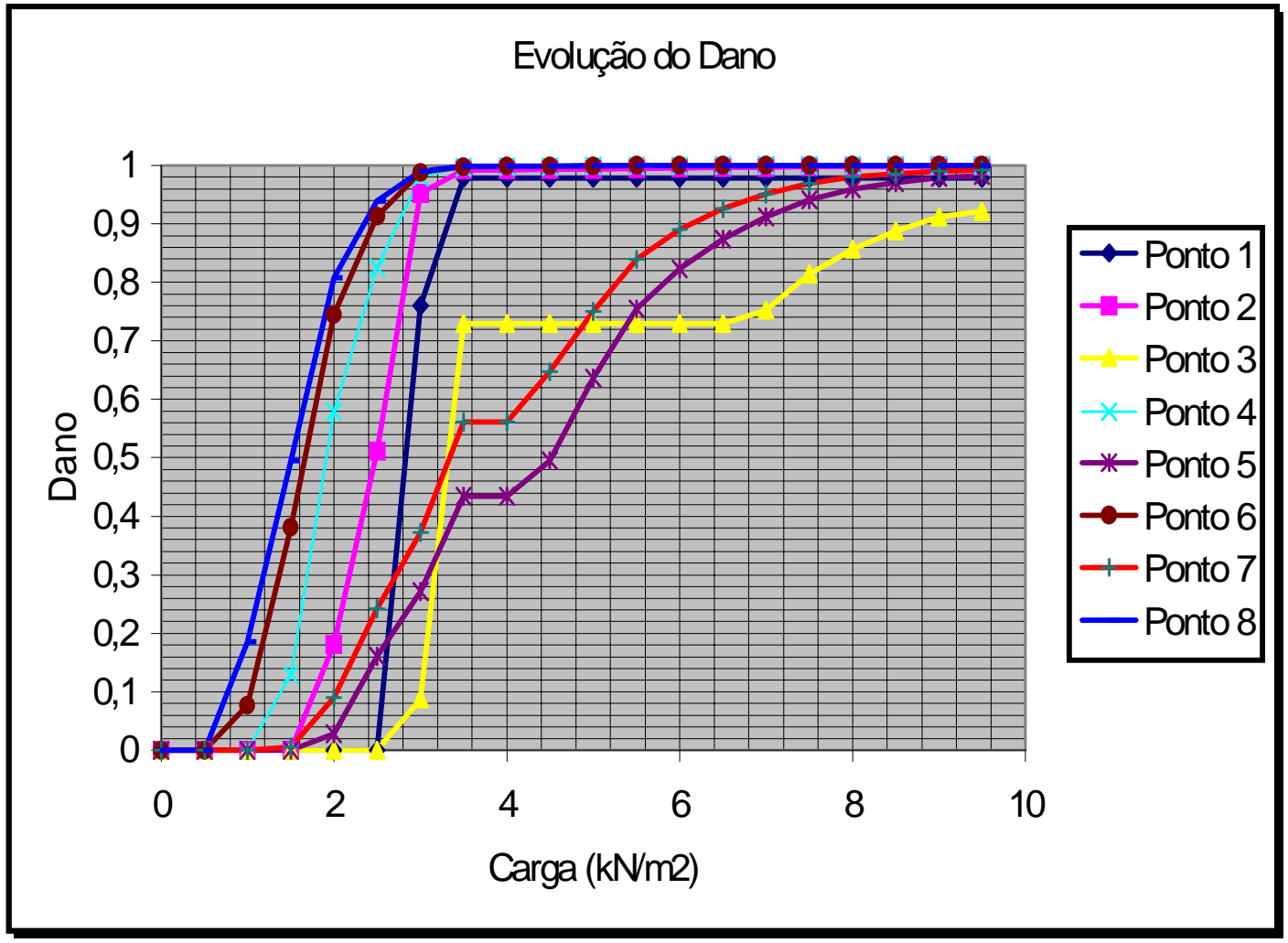

FIGURA 5.15. Evolução do dano nos pontos de Gauss Ex. 2

Da fig. 5.15 observa-se que o processo de danificação na seção transversal inicia-se pelo ponto de Gauss número 8 (ponto mais solicitado à tração), estendendose para os pontos $6,4,2,7,5,1$ e 3 , à medida que o carregamento se aproxima do valor máximo $\left(9,5 \mathrm{kN} / \mathrm{m}^{2}\right)$.

Entre 0 e 2,0kN/m ${ }^{2}$ os pontos de Gauss abaixo da superfície média da laje (pontos 2, 4, 6 e 8) têm todos dano inferiores a 0,81 e os pontos acima daquela superfície (pontos $1,3,5$ e 7) têm dano inferiores a 0,1 (praticamente íntegros). Da fig. 5.14, observa-se ser este intervalo correspondente ao trecho aproximadamente linear.

Entre $2,0 \mathrm{kN} / \mathrm{m}^{2}$ e $3,0 \mathrm{kN} / \mathrm{m}^{2}$ os pontos $2,4,6$ e 8 tendem para dano iguais a 1.0 e os pontos 3,5 e 7 têm todos dano inferiores a 0,4. Da fig. 5.14 observa-se que neste intervalo a tangente à curva carga-deslocamento para a discretização em 144 células sofre grande variação. Entre $3,0 \mathrm{kN} / \mathrm{m}^{2}$ e $9,0 \mathrm{kN} / \mathrm{m}^{2}$ não há grande variação 
desta tangente e os pontos $1,3,5$ e 7 têm danos próximos de 1,0, indicativo de que a estrutura está próxima do colapso.

Acima de $9,0 \mathrm{kN} / \mathrm{m}^{2}$, todos os pontos de Gauss têm dano superiores a 0,9. Da experimentação realizada por CAMPOS (2000), quando tentou-se impor à laje o carregamento de $9,25 \mathrm{kN} / \mathrm{m}^{2}$, ocorreu a ruptura. Assim, para a análise numérica, pode-se estabelecer que quando todos os pontos de Gauss alcançarem o valor de dano 0,9 , tem-se esgotada toda a capacidade resistente da seção transversal.

Quanto às armaduras, as deformações relativas ao ponto central, no ponto mais próximo da borda inferior da laje estão tabelados a seguir. Como no caso experimental o extensômetro já registrava uma deformação inicial de $0.0070 \%$ para o carregamento nulo, devida ao peso próprio, tentou-se, no presente estudo, computá-la aplicando-se 4 incrementos de carga iniciais de $0.4688 \mathrm{kN} / \mathrm{m}^{2}$ relativos ao peso próprio e a seguir, mais 19 incrementos relativos ao carregamento externo.

Os valores obtidos são dados na tabela a seguir e representados na fig. 5.16.

\begin{tabular}{|c|c|c|}
\hline \multicolumn{3}{|c|}{ Deformações na armadura mais próxima da borda inferior $\left(\% \%_{00}\right)$} \\
\hline Carga $\left(\mathrm{Kn} / \mathbf{m}^{2}\right)$ & Experimental & M.E.C. (144 Células) \\
\hline 0.00 & 0.0070 & 0.091 \\
\hline 0.50 & $\mathbf{0 . 0 3 0 0}$ & 0.180 \\
\hline 1.00 & $\mathbf{0 . 0 8 5 0}$ & 0.425 \\
\hline 1.50 & 0.1520 & 0.672 \\
\hline 2.00 & 0.2810 & 1.25 \\
\hline 2.50 & 1.7030 & 1.45 \\
\hline $\mathbf{3 . 0 0}$ & 2.0960 & 1.57 \\
\hline 3.50 & 2.4220 & 2.25 \\
\hline 4.00 & 2.7900 & 2.41 \\
\hline 4.50 & 3.2840 & 2.66 \\
\hline 5.00 & $\mathbf{3 . 8 3 7 0}$ & 2.92 \\
\hline 5.50 & 4.3410 & 3.15 \\
\hline 6.00 & 5.2180 & 3.48 \\
\hline 6.25 & 5.5680 & - \\
\hline 6.50 & - & 3.74 \\
\hline 7.00 & - & 3.99 \\
\hline 7.50 & - & 4.27 \\
\hline 8.00 & - & 4.52 \\
\hline 8.50 & - & 4.76 \\
\hline 9.00 & - & 4.89 \\
\hline
\end{tabular}




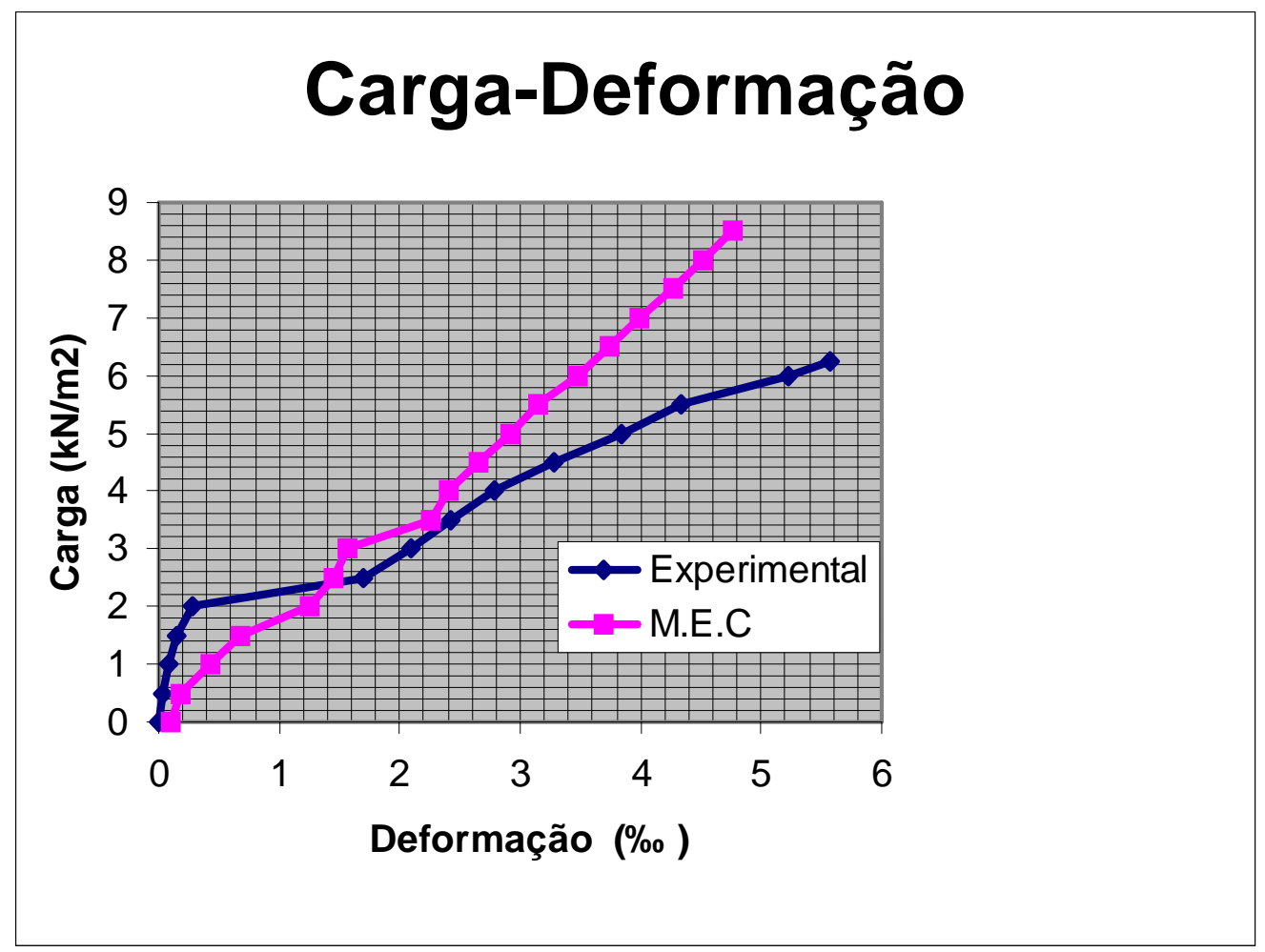

FIGURA 5.16. Curvas Carga - Deformação na armadura

Veja-se que até o carregamento de $2,5 \mathrm{kN} / \mathrm{m}^{2}$ as deformações na armadura pelo presente estudo foram maiores do que aquelas verificadas experimentalmente; acima deste valor de carregamento, as deformações obtidas experimentalmente foram maiores. Esta inversão deve-se ao fato de que o processo de fissuração do concreto e conseqüente transferência de esforços deste para o aço acarreta maior solicitação (e deformação) na armadura, o que, segundo a formulação aqui desenvolvida não ocorre de forma tão acentuada; perceba-se que na curva experimental, entre $2,0 \mathrm{kN} / \mathrm{m}^{2}$ e $2,5 \mathrm{kN} / \mathrm{m}^{2}$ há um patamar que não fica evidente na curva relativa ao presente estudo. Perceba-se, ainda, que para o carregamento de serviço $\left(3,5 \mathrm{kN} / \mathrm{m}^{2}\right)$ a deformação na armadura obtida no presente estudo foi $7 \%$ inferior àquela verificada experimentalmente. Já para o carregamento de $6,0 \mathrm{kN} / \mathrm{m}^{2}$ esta diferença subiu para $33 \%$.

Os resultados experimentais relativos à deformação na armadura só foram tabelados por CAMPOS (2000) até o valor de $6,25 \mathrm{kN} / \mathrm{m}^{2}$, quando a armadura alcançou a tensão de escoamento (relativa à deformação permanente de 0.002). 


\section{c. Exemplo 3.}

Analisa-se a seguir uma laje cogumelo de dimensões $1200 \mathrm{~cm} \times 1200 \mathrm{~cm}$ apoiada sobre nove pilares de seções transversais $20 \mathrm{~cm} \times 20 \mathrm{~cm}$, como ilustra a fig.5.17. A fim de se evitar prolixidade, o estudo foi dirigido para a região do pilar central, P5. Altura da laje: $h=20 \mathrm{~cm}$; altura útil $d=18,5 \mathrm{~cm}$.

Dados relativos ao Concreto: $\mathbf{f}_{\mathbf{c k}}=37,3 \mathrm{MPa}$; Módulo de Elasticidade: 42.160MPa; Coeficiente de Poisson: 0,20.

Dados relativos à armadura longitudinal: Módulo de elasticidade: 210.000MPa; Parâmetro de endurecimento: 21.000MPa; tensão de escoamento: 670MPa.

A distribuição da armadura longitudinal está apresentada na tabela a seguir.

\begin{tabular}{|l|l|c|c|c|}
\hline & Região do pilar central & $\begin{array}{c}\text { Região dos pilares P2, } \\
\text { P4, P6 e P8 }\end{array}$ & $\begin{array}{c}\text { Região dos pilares P1, } \\
\text { P3, P7 e P9 }\end{array}$ & Demais regiões \\
\hline Armadura de tração & $31 \phi 16 \mathrm{~mm} \mathrm{c} / 10 \mathrm{~cm}$ & $15 \phi 16 \mathrm{~mm} \mathrm{c} / 20 \mathrm{~cm}$ & $10 \phi 16 \mathrm{~mm} \mathrm{c} / 25 \mathrm{~cm}$ & $\phi 16 \mathrm{~mm} \mathrm{c} / 20 \mathrm{~cm}$ \\
\hline Armad. de compressão & $21 \phi 8 \mathrm{~mm} \mathrm{c} / 15 \mathrm{~cm}$ & $21 \phi 8 \mathrm{~mm} \mathrm{c} / 15 \mathrm{~cm}$ & $21 \phi 8 \mathrm{~mm} \mathrm{c} / 15 \mathrm{~cm}$ & - \\
\hline
\end{tabular}

Armadura transversal:

Na região do pilar central: $\phi 10 \mathrm{~mm}$ a cada $10 \mathrm{~cm}$; tensão de escoamento: 430MPa;

Tanto as armaduras longitudinais quanto as armaduras transversais têm mesmos valores nas direções $\mathbf{x}$ e $\mathbf{y}$.

Carregamento: tentou-se impor à estrutura um carregamento total de $15.0 \mathrm{kN} / \mathrm{m}^{2}$, em 15 incrementos de carga de $1.0 \mathrm{kN} / \mathrm{m}^{2}$. Tolerância de convergência do processo iterativo: $0,01 \%$.

O contorno foi discretizado em 48 elementos e o domínio em 272 células triangulares. 


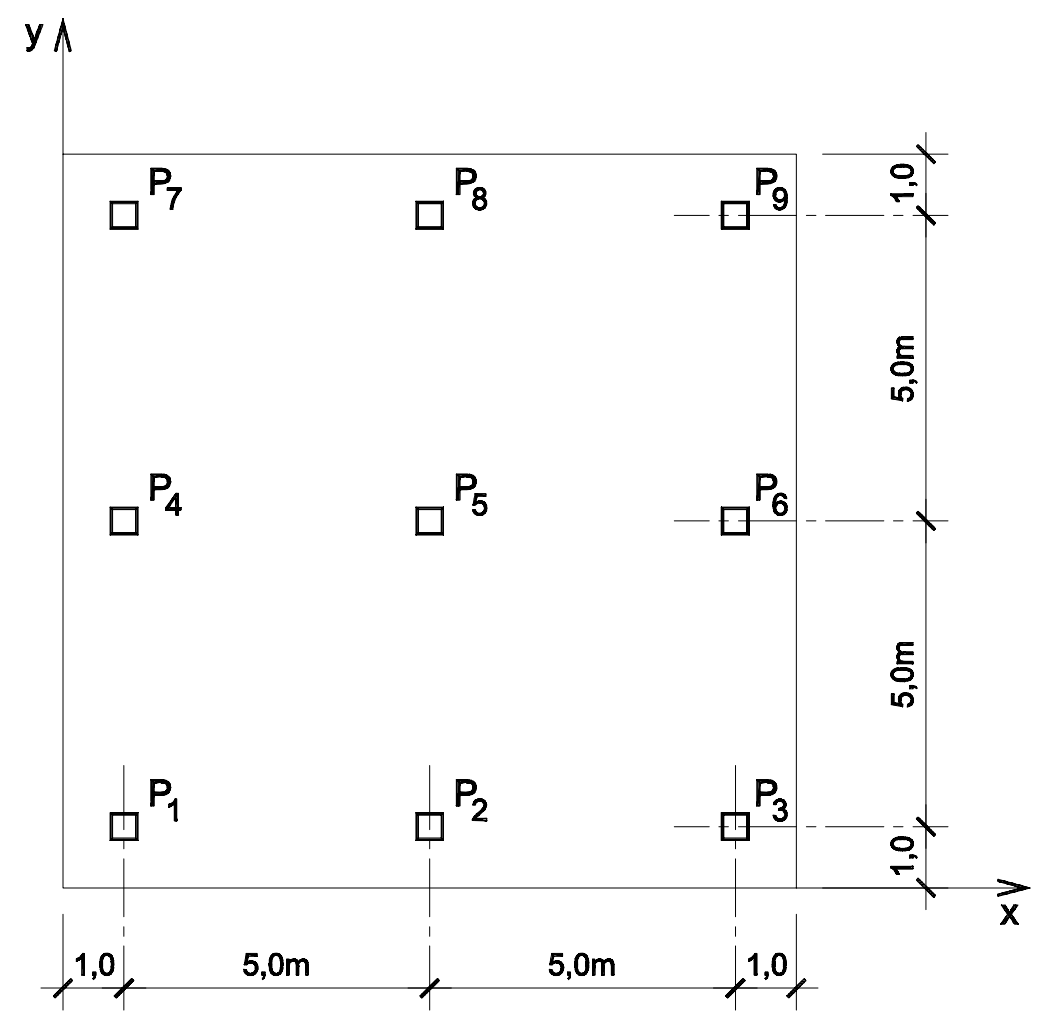

FIGURA 5.17. Laje Cogumelo sobre 9 pilares

A seguir apresentam-se os resultados obtidos no presente estudo:

- Momentos até o carregamento de $10 \mathrm{kN} / \mathrm{m}^{2}$ no ponto central da laje:

\begin{tabular}{|c|c|c|}
\hline \multirow{2}{*}{ Incremento } & \multicolumn{2}{|c|}{ Momentos $(\mathrm{kNxcm})$} \\
\cline { 2 - 3 } & $\mathrm{M}_{\mathrm{xx}}$ (elástico) & $\mathrm{M}_{\mathrm{xx}}$ (não linear) \\
\hline 1 & 12.716 & 12.179 \\
\hline 2 & 25.432 & 19.317 \\
\hline 3 & 38.148 & 23.570 \\
\hline 4 & 50.864 & 26.263 \\
\hline 5 & 63.580 & 28.110 \\
\hline 6 & 76.296 & 29.921 \\
\hline 7 & 89.012 & 31.530 \\
\hline 8 & 101.72 & 32.968 \\
\hline 9 & 114.44 & 34.247 \\
\hline 10 & 127.16 & 35.384 \\
\hline
\end{tabular}

Na fig.5.18 está representada a evolução dos momentos em função dos incrementos de carga. 


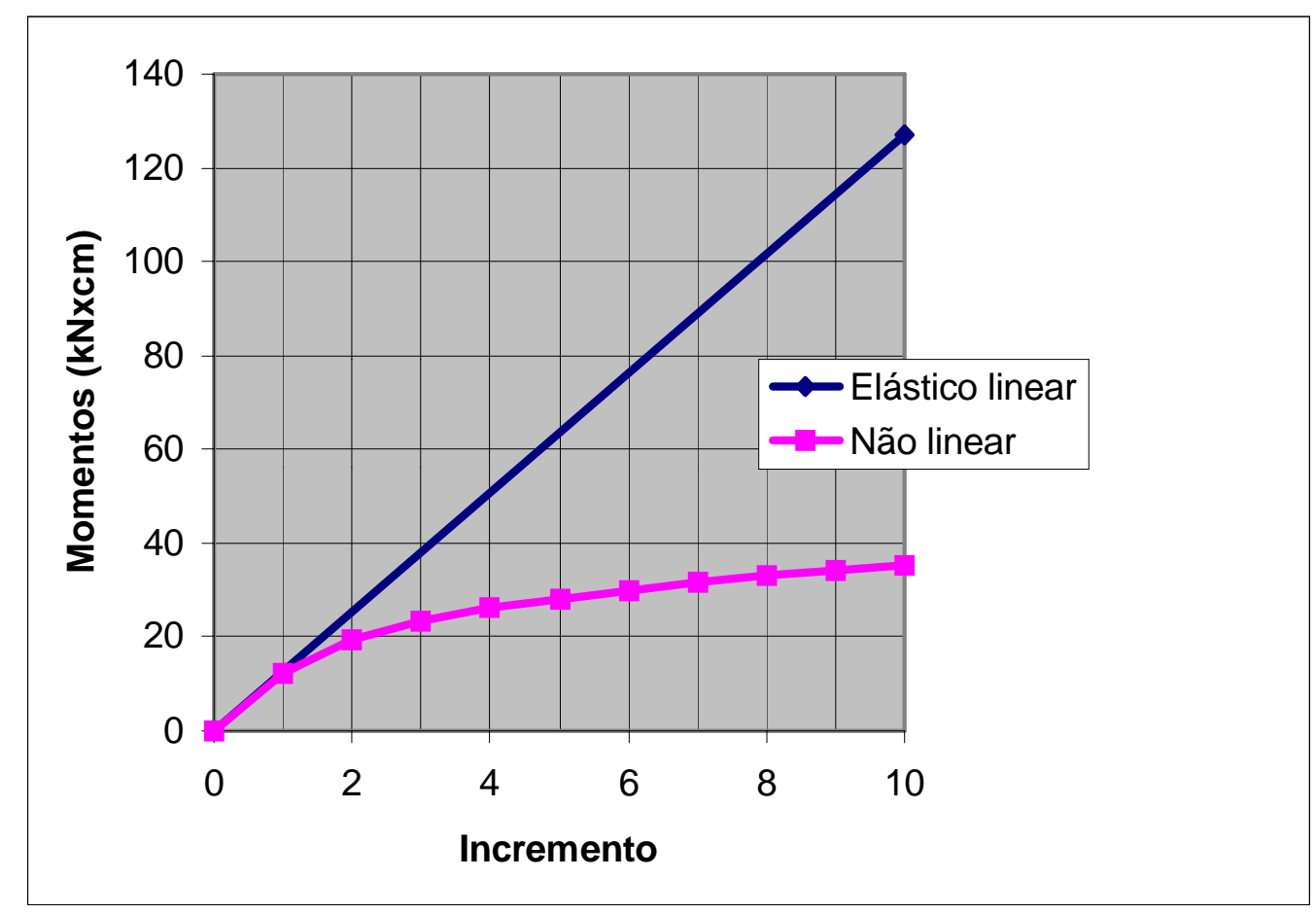

FIGURA 5.18. Curvas Incrementos - Momentos

- Reações elásticas nos pilares para o carregamento de $10 \mathrm{kN} / \mathrm{m}^{2}$ :

\begin{tabular}{|c|c|c|c|c|c|c|c|c|}
\hline \multicolumn{10}{|c|}{ Reações (kN) } \\
\hline P1 & P2 & P3 & P4 & P5 & P6 & P7 & P8 & P9 \\
\hline 28.91 & 229.36 & 28.91 & 229.36 & 443.29 & 229.36 & 28.91 & 229.36 & 28.91 \\
\hline
\end{tabular}

- Evolução do dano no ponto central da laje até o incremento 14:

Foram adotados 12 pontos de Gauss ao longo da espessura da laje, cujas ordenadas tem os seguintes valores:

\begin{tabular}{|c|c|c|c|c|c|c|c|c|c|c|c|}
\hline \multicolumn{10}{|c|}{ ORDENADAS DOS PONTOS DE GAUSS (cm) } \\
\hline \multicolumn{10}{|c|}{ PONTOS } \\
\hline 1 & 2 & 3 & 4 & 5 & 6 & 7 & 8 & 9 & 10 & 11 & 12 \\
\hline 1.25 & -1.25 & $\mathbf{3 . 6 8}$ & $\mathbf{- 3 . 6 8}$ & $\mathbf{5 . 8 7}$ & $\mathbf{- 5 . 8 7}$ & $\mathbf{7 . 7 0}$ & $-\mathbf{7 . 7 0}$ & $\mathbf{9 . 0 4}$ & $\mathbf{- 9 . 0 4}$ & $\mathbf{9 . 8 2}$ & $\mathbf{- 9 . 8 2}$ \\
\hline
\end{tabular}

Para estes pontos, tem-se a evolução do dano conforme a tabela seguinte. 


\begin{tabular}{|c|c|c|c|c|c|c|c|c|c|c|c|c|}
\hline \multicolumn{13}{|c|}{ Dano } \\
\hline \multirow{2}{*}{$\begin{array}{c}\text { Increme } \\
\text { nto }\end{array}$} & \multicolumn{12}{|c|}{ Pontos de Gauss } \\
\hline & 1 & 2 & 3 & 4 & 5 & 6 & 7 & 8 & 9 & 10 & 11 & 12 \\
\hline 1 & 0.0000 & 0.0000 & 0.0000 & 0.0000 & 0.0000 & 0.0000 & 0.1227 & 0.0000 & 0.2186 & 0.0000 & 0.2687 & 0.0000 \\
\hline 2 & 0.0000 & 0000 & 0.0954 & $\overline{0000}$ & 0.3786 & 0.0000 & 0.5421 & 0.0000 & 0.6325 & .0469 & 0.6757 & 0.0754 \\
\hline 3 & $\overline{1000}$ & 0000 & 0.3393 & $\overline{0000}$ & 0.6178 & 0.0376 & 0.7529 & 0.1323 & 0.8182 & 1898 & 0.8467 & 0.2196 \\
\hline 4 & 0000 & 0000 & 0.5161 & 0.0000 & 0.7614 & 0.1392 & 0.8615 & .2368 & 0.9040 & .2950 & 0.9211 & .3256 \\
\hline 5 & 071 & 0000 & 0.6437 & 0.0542 & 0.8475 & 0.2205 & 0.9178 & 0.3193 & 0.9443 & 0.3779 & 0.9543 & 0.4075 \\
\hline 6 & 115 & $\overline{0000}$ & 0.7358 & 0.1189 & 0.8992 & 0.2880 & 0.9475 & 3866 & 0.9641 & $\overline{4441}$ & 0.9701 & 04729 \\
\hline 7 & 0.2043 & 0.0000 & 0.8023 & 0.1747 & 0.9307 & 0.3452 & 0.9637 & 0.4456 & 0.9745 & 0.4985 & 0.9784 & 0.5264 \\
\hline 8 & 873 & 0000 & 0.8505 & 2239 & 0.9501 & 0.3944 & 0.9731 & 0.4900 & 0.9805 & .5443 & 0.9833 & 0.3524 \\
\hline 9 & 3613 & $\overline{0000}$ & 0.8854 & 0.2676 & 0.9624 & 0.4373 & 0.9789 & 0.5307 & $\overline{0.9844}$ & .5834 & 0.9866 & 0.6094 \\
\hline 10 & 0.4273 & $\overline{0000}$ & 0.9108 & 0.3068 & 0.9705 & 0.4750 & 0.9828 & 0.5661 & 0.9872 & 0.6173 & $\mathbf{0 . 9 8 9 0}$ & 0.6425 \\
\hline 11 & 0.4862 & 0.0000 & 0.9293 & 0.3423 & 0.9759 & 0.5083 & 0.9856 & 0.5973 & 0.9893 & 0.6470 & 0.9909 & 0.6716 \\
\hline 12 & 0.5387 & 0.0000 & 0.9430 & 0.3745 & 0.9798 & 0.5381 & 0.9877 & 0.6250 & 0.9910 & 0.6735 & 0.9924 & 0.6974 \\
\hline 13 & 0.5856 & 0.0177 & 0.9532 & 0.4039 & 0.9827 & 0.5650 & 0.9895 & 0.6499 & 0.9924 & 0.6973 & 0.9936 & 0.7207 \\
\hline 14 & 0.6274 & 0.0437 & 0.9608 & 0.4309 & 0.9850 & 0.5893 & 0.9910 & 0.6724 & 0.9936 & $\mathbf{0 . 7 1 8 8}$ & 0.9947 & 0.7216 \\
\hline
\end{tabular}

Estes resultados estão representados na fig. 5.19:

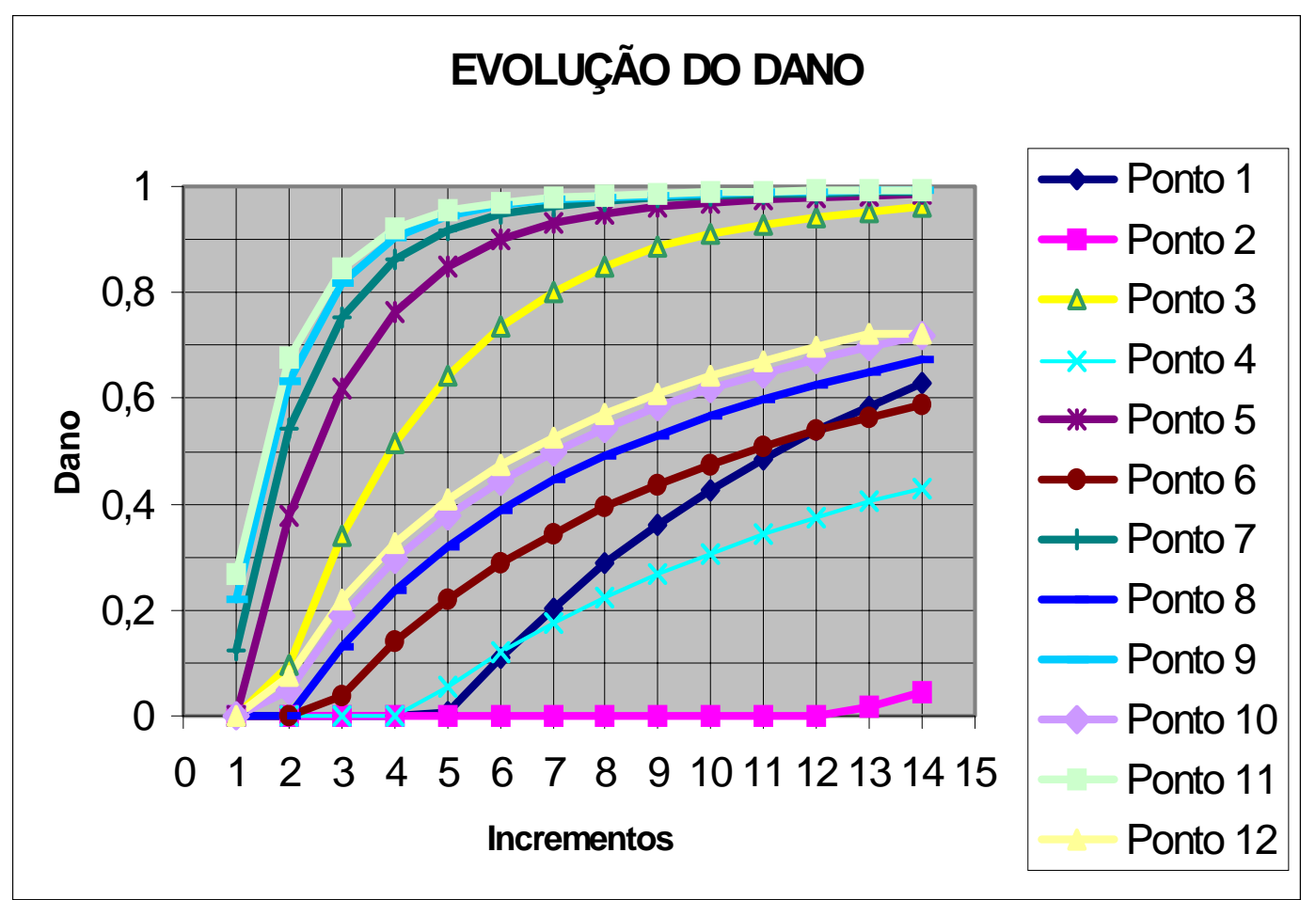

FIGURA 5.19. Evolução do dano nos pontos de Gauss - ex. 3

Perceba-se que neste caso os pontos de Gauss onde inicia-se o processo de danificação são aqueles acima da superfície média. 
- Deformações nas armaduras longitudinais de tração (cota 5,9cm) e de compressão (cota $-8,5 \mathrm{~cm})$ no ponto central da laje:

\begin{tabular}{|c|c|c|}
\hline \multicolumn{3}{|c|}{ Deformações $(\%)$} \\
\hline Incremento & Armad . compressão & Armad. tração \\
\hline 1 & -0.031 & 0.021 \\
\hline 2 & -0.062 & 0.043 \\
\hline 3 & -0.093 & 0.064 \\
\hline 4 & -0.124 & 0.086 \\
\hline 5 & -0.155 & 0.108 \\
\hline 6 & -0.187 & 0.130 \\
\hline 7 & -0.219 & 0.152 \\
\hline 8 & -0.251 & 0.174 \\
\hline 9 & -0.283 & 0.196 \\
\hline 10 & -0.316 & 0.219 \\
\hline 11 & -0.348 & 0.242 \\
\hline 12 & -0.381 & 0.265 \\
\hline 13 & -0.414 & 0.288 \\
\hline 14 & -0.447 & 0.310 \\
\hline
\end{tabular}

$\mathrm{Na}$ fig. 5.20 encontram-se esquematizadas as curvas incremento deformação relativas à tabela anterior.

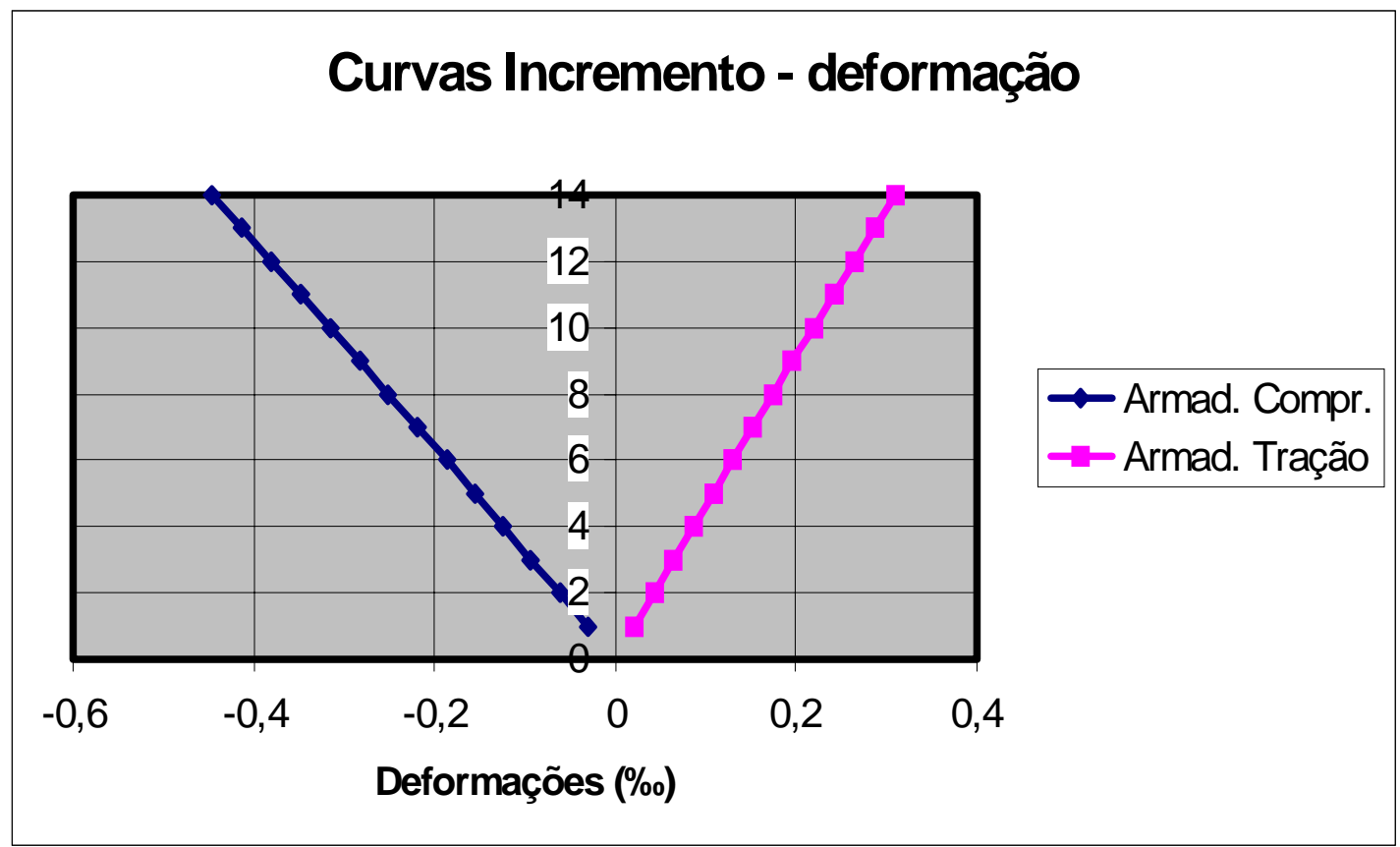

FIGURA 5.20. Curvas incremento - Deformação nas armaduras 
- Tensão normal na armadura de cisalhamento (estribo):

Os valores tabelados a seguir são relativos ao ponto distante $8 \mathrm{~cm}$ da face do pilar central, na cota $(582 \mathrm{~cm} ; 600 \mathrm{~cm})$, ponto este onde as tensões cisalhantes, na direção x, apresentam maiores valores. Tentou-se aplicar 15 incrementos de carga mas não foi possível, uma vez que a tensão no estribo ultrapassou o limite elástico no último incremento (incremento 15).

\begin{tabular}{|c|c|c|c|c|c|c|c|c|c|c|c|c|c|}
\hline \multicolumn{14}{|c|}{ Tensão na armadura Transversal - $\sigma_{\mathrm{xx}}(\mathrm{MPa})$} \\
\hline \multicolumn{14}{|c|}{ Incrementos } \\
\hline 1 & 2 & 3 & 4 & 5 & 6 & 7 & 8 & 9 & 10 & 11 & 12 & 13 & 14 \\
\hline $\mathbf{0 . 0 3}$ & 4.36 & 15.70 & 33.81 & $\mathbf{5 5 . 6 3}$ & 80.16 & 111.0 & 150.3 & 191.7 & 235.0 & 279.8 & 325.9 & 373.1 & 421.2 \\
\hline
\end{tabular}

A fig. 5.21 ilustra o crescimento da tensão normal no estribo, com o aumento da carga.

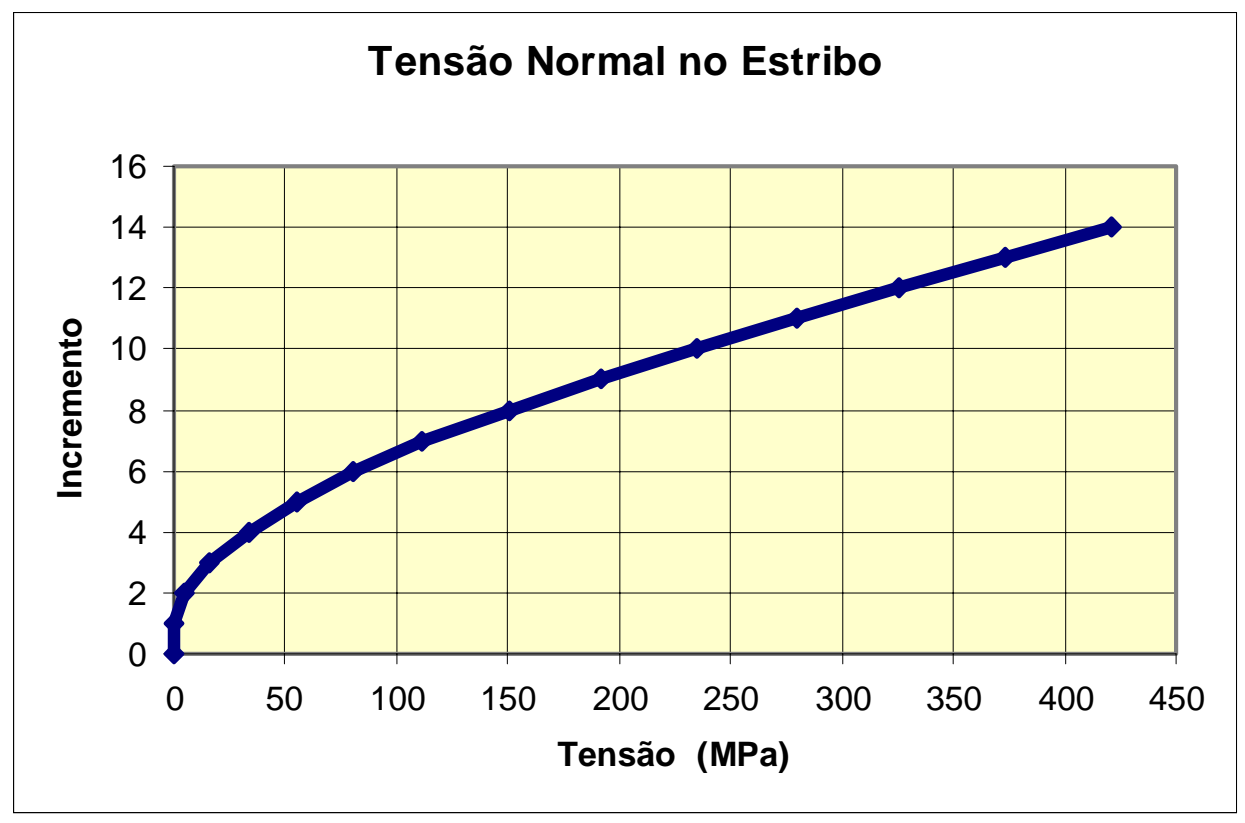

FIGURA 5.21. Tensão normal no estribo 
- Esforço Cortante no concreto e na armadura ( dir. x):

Os resultados estão na tabela a seguir onde: Inc.=incremento; Con= concreto; Arm $=$ armadura

\begin{tabular}{|c|c|c|c|c|c|c|c|c|c|c|c|c|c|c|}
\hline & \multicolumn{10}{|c|}{ Esforço cortante $(\mathrm{kN})$} \\
\hline Inc. & 1 & 2 & 3 & 4 & 5 & 6 & 7 & 8 & 9 & 10 & 11 & 12 & 13 & 14 \\
\hline Con. & 0.38 & 0.73 & 1.03 & 1.28 & 1.50 & 1.70 & 1.86 & 1.95 & 2.02 & 2.09 & 2.14 & 2.18 & 2.21 & 2.24 \\
\hline Arm. & 0.00 & 0.03 & 0.12 & 0.25 & 0.41 & 0.59 & 0.82 & 1.11 & 1.42 & 1.74 & 2.07 & 2.41 & 2.76 & 3.11 \\
\hline
\end{tabular}

Como pode-se observar das fig. 5.19 e 5.22, à medida em que o processo de danificação no concreto aumenta, diminui sua capacidade de absorção do esforço cortante, e aumenta a parcela deste esforço absorvida pelo estribo.

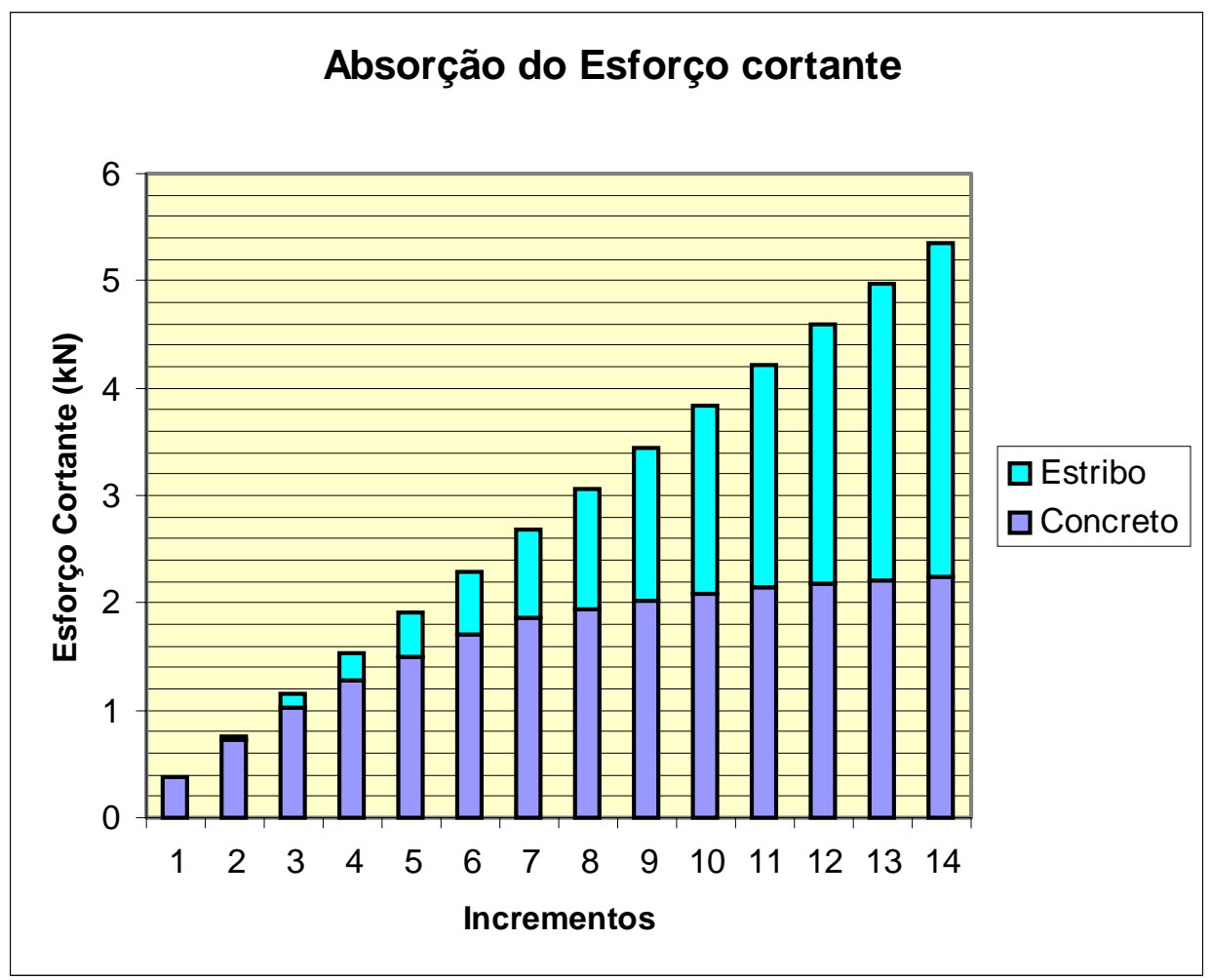

FIGURA 5.22. Absorção do esforço cortante (dir. $x)$

Como pode-se observar da fig. 5.19, no incremento 14, os pontos de Gauss acima da superfície média, com exceção do ponto 1 , têm todos danos superiores a 0,9 ; os pontos abaixo desta superfície, têm danos inferiores a 0,80 ; ademais, as armaduras longitudinais estão em regime elástico linear, com deformações abaixo de 
0,5\%o (fig. 5.20); após o incremento 14, a armadura transversal ultrapassou o limite elástico; por inferência, deduz-se que, em se aumentando a área da armadura transversal, aumenta-se a capacidade resistente da seção transversal em análise. Pode-se, ainda mantendo-se aquela área de armadura transversal, reduzir a área da seção transversal da armadura longitudinal até que se obtenham deformações próximas de $-3,5 \%$ e 10,0\%o para as armaduras de compressão e tração, respectivamente; com isto, no incremento 14 (correspondente a $14 \mathrm{kN} / \mathrm{m}^{2}$ ), ter-se-ia chegado à capacidade resistente da seção transversal em análise. 


\section{CAPÍtULO Vi}

\section{CONCLUSÕES}

O presente trabalho tratou de uma formulação utilizando-se o Método dos Elementos de Contorno para a análise de pavimentos, com ênfase na análise de lajes cogumelo. Utilizou-se a teoria de placas de Reissner, a qual mostrou-se eficiente, tanto para a análise de placas esbeltas quanto para placas moderadamente espessas, uma vez que considera as deformações por cisalhamento transversal.

A formulação desenvolvida possibilitou a consideração de carregamento uniformemente distribuído em todo o domínio, ou parte dele, cargas transversais e momentos distribuídos em linha e cargas concentradas. Considerou-se, ainda, a possibilidade de ocorrência de um campo de momentos iniciais, o que tornou possível a análise não linear (não linearidade física), imprescindível à análise de estruturas de concreto.

A formulação de placas com enrijecedores permitiu a consideração de apoios pontuais, em áreas discretas e em linhas. Este último baseado numa combinação com o Método dos Elementos Finitos. Este modelo proporcionou uma avaliação precisa dos momentos e forças cortantes nas conexões entre placa e elementos lineares (vigas).

Quanto ao ponto de colocação (ponto fonte), foi adotada a formulação regular, evitando possíveis erros na determinação dos valores principais de integrais onde ocorrem singularidades.

Para a avaliação das tensões e deformações ao longo da altura das seções transversais, adotou-se um modelo estratificado. Com isso pôde-se proceder uma análise distinta para o concreto e o aço. Para o concreto, adotou-se o modelo de dano de Mazars, considerando-se, também, a influência das deformações tangenciais. Para o aço foi adotado um modelo elastoplástico uniaxial com endurecimento isotrópico. 
Quanto à absorção das tensões tangenciais, o modelo foi baseado na analogia da treliça clássica de Mörsch. Isto permitiu a quantificação das parcelas de tensões absorvidas pelo concreto e pelas armaduras transversais.

Com os exemplos ilustrativos, fica comprovada a adequação da formulação à análise de estruturas de pavimentos de edifícios e, em particular, à análise de lajes cogumelo onde há efeito considerável de concentração de tensões. Puderam ser avaliados a resposta carga-deslocamento nos pontos críticos da estrutura; o processo de danificação ao longo dos pontos de Gauss; as deformações nas armaduras longitudinais de tração e compressão; a evolução na absorção do esforço cortante pelo concreto e pelo aço, dentre outros. A eficiência da formulação proposta pôde ser verificada, também, quando se compararam alguns resultados com os resultados obtidos com outras formulações, e em ensaios experimentais.

Quanto ao prosseguimento da pesquisa, algumas das medidas que podem ser implementadas são:

- atualização das matrizes envolvidas, a cada iteração, visando-se maior rapidez quanto ao processo de convergência;

- integração sobre o contorno das células, proporcionando maior exatidão dos cálculos envolvidos na consideração de placas com campo de momentos iniciais;

- consideração da não linearidade física sobre os elementos de viga; isto possibilita uma análise mais completa de pavimentos convencionais em concreto armado;

- utilização de outros modelos constitutivos para o aço e o concreto;

- cálculo das reações nos pilares quando se considera a não linearidade física, no caso de lajes cogumelo;

- análise experimental de lajes cogumelo, podendo-se melhor situar as respostas obtidas na presente formulação. 


\section{BIBLIOGRAFIA}

ABRAMOWITZ, M.; STEGUN, I.A. Handbook of Mathematical Functions. N. Y.. Dover Publications, 1965.

ALIABADI, M.H.; BREBBIA,C.A.; RASHED, Y.F. On the evaluation of the stresses in the BEM for Reissner plate-bending problems. Appl. Math. Modelling. v. 21, p.155-163, March, 1997.

ALTIERO, N.J.; SIKARSKIE, D.L. A boundary integral method applied to plates of arbitrary plan form. Computers \& Structures. v.9, p.163-168, 1978.

ÁLVARES, M.S. Contribuição ao estudo e emprego de modelos simplificados de dano e plasticidade para a análise de estruturas de barras em concreto armado. Tese(Doutorado). São Carlos, 1999. Escola de Engenharia de São Carlos, Universidade de São Paulo.

ÁLVARES, M.S. Estudo de um modelo de dano para o concreto: formulação, identificação paramétrica e aplicação com emprego do Método dos Elementos Finitos. Dissertação (mestrado). São Carlos, 1993. Escola de Engenharia de São Carlos, Universidade de São Paulo.

ARGYRIS, J.H.; KELSET, S. Energy theorems and structural analysis. London, Butteworths, 1960.

BARCELLOS, C.S.; SILVA, L.H.M. A boundary element formulation for de Mindlin's plate model; in: BREBBIA,C.A.; VENTURINI, W.S. eds. Boundary Element Techniques. Applications in Stress Analysis and Heat Transfer. Southampton. C.M. Publ., 1987.

BARRET, K.E.; ELLIS, S. An exact theory of elastic plates. Int. J. Solids Struct. v.24(9), p.859-880, 1988.

BECKER, A.A. The Boundary Element Method in Engineering. England, McGRAW-HILL Book Conpany. Europe, 1992.

BETTY, E. Teoria dell'elasticità. Il Nuovo Ciemento. p. 7-10, 1872.

BEZINE, G.P.; GAMBY, D.A. A new integral equation formulation for plate bending problems. In: Brebbia, C.A. ed. Recent advances in BEM. p. 327-342, Pentech Press, 1978.

BUSSAMRA, F. L. S. Equações constitutivas do concreto baseadas na mecânica do dano contínuo, 1993. Dissertação (Mestrado). Escola Politécnica da Universidade de São Paulo.

CAMP, C.V.; GIPSON, G.S. Biharmonic analysis of rectilinear plates by Boundary Element Method. Int. J. Num. Meth. Engng. n.30, p.517-539.

CAMPOS, C. O. Análise experimental de lajes de concreto armado reforçadas pela face superior, 2000. Dissertação (Mestrado). Universidade Federal de Goiás. Escola de Engenharia Civil.

CERVENKA, V. Inelastic Finite Element analysis of reinforced concrete panels under in plane loads. Ph.D thesis. University of Colorado, 1970.

CHEN, A.C.T.; CHEN, W.F. Constitutive Relations for concrete. Journal of Engineering Mechanics Division, ASCE, v.101, n. n.EM4, p. 465-481, August, 1975.

CHEN, W.F.; HAN, D.J. A nonuniform hardening plasticity model for concrete Materials. Mech. of Mat. v.4, p.283-302, 1985. 
CHEN, W.F.; HAN, D.J. Plasticity for Structural Engineers. Springer - Verlag, N. Y., 1988.

CHUEIRI, L.H.M. Formulação do Método dos Elementos de Contorno para análise elastoplástica de placas. São Carlos, 1994. Tese (doutorado) - Escola de Engenharia de São Carlos, Universidade de São Paulo.

DAMJANIC, F.; FIGUEIRAS, J. A.; OWEN, D.R.J. Finite Element analysis of reinforced and prestressed concrete structures including thermal load. Computer Methods in applied Mechanics and Engineering. v. 41, p.323-366, 1983.

DEBBIH, M.; EL-ZAFRANY, A.; FADHIL, S. An efficient approach for Boundary Element bending analysis of thin and thick plates. Computers \& Structures, v. 56, p.565-576, 1995.

DEBBIH, M.; EL-ZAFRANY, A.; FADHIL, S. Boundary Element analysis of thick Reissner plates in bending. Engineering Analysis. v. 14, p.159-169, 1995.

EL-ZAFRANY, A. Boundary Element analysis of thich Reissner plates in bending. Ed. M.H. Aliabadi, Computational Mechanics Publications, London, 1998.

FERNANDES, G. R. O método dos elementos de contorno aplicado à análise nãolinear de placas. Dissertação (Mestrado). São Carlos, 1998. Escola de Engenharia de São Carlos, Universidade de São Paulo.

FIGUEIRAS, J.A. Ultimate Load analysis of anisotropic and reinforced concrete plates and shells. Tese (Doutorado). Swansea, 1983. University College of Swansea.

FLÓREZ-LÓPEZ, J. Modelos de daño concentrado para la simulation numerica del colapso de pórticos planos. Revista Internacional de Métodos Numéricos para Cálculo y Diseno en Ingenieria, v.9, n.2, p. 123-129, 1993.

GUO-SHU, S. MUKERJEE, S. Boundary Element Method analysis of bending plates of arbitrary chape with general boundary conditions. Engng. Analysis. v.3(1), p.36-44, 1986.

HAND, F.R.; PECKNOID, D.A.; SCHNOBRICH, W.C. Nonlinear layered analysis of RC plates and shells. J. Struct. Div., ASCE, v. 99, n.ST7, p. 1491-1505, July, 1973.

HANSEN, E.B. Numerical solution of integro-differential and singular integral equations for plate bending problems. J. of Elasticity. v.6(1), p.39-56, 1976.

HARTLEY, G.A.; ABDEL-AKHER, A. Analytic ingration procedures for plate bending analysis, in: BREBBIA, C.A. ed.. Boundary elements X. C.M. Publ. v.3, p. 391-405, 1988.

HARTLEY, G.A.; ABDEL-AKHER, A. Boundary integration and interpolation procedures for plate bending. Int. Num. Meth. Engng. v.28(6), p.1389-1408, 1989.

HARTLEY, G.A.; AHMED, A.A. Evaluation of boundary integrals for plate bending. Int. Num. Meth. Engng. v.28(2), p.75-93, 1989.

HARTMANN, F.; ZOTEMANTEL, R. The direct Boundary Element Method in plate bending. Int. J. Num. Meth. Engng. v.23(11), p.2049-2069, 1986.

HU, T.H.; SCHNOBRICH, W.C. Constitutive modeling of concrete by using nonassociated plasticity. Journal of Materials in Civil Engineering. v.1, n.4, p. 199-216, November, 1989.

HU, T.H.; SCHNOBRICH, W.C. Non linear analysis of cracked reinforced concrete. ACI Structural Journal, v.87, n.2, p.199-207, March-April, 1990.

JASWON, M.A.; MAITI, M.; SYMM, G.J. Numerical biharmonic analysis and some applications. Int. J. Solids Struct. v.3, p.309-332, 1967. 
JOFRIET, J.C.; McNEICE, G.M. Finite Element analysis of reinforced concrete slabs. J. Struct. Div., ASCE, n. 97, p. 785-806, MAR., 1971.

KACHANOV, L.M. Time of the rupture process under creep conditions. T.V.Z. Akad. S.S.R. Tech. Nauk., 8, p.26-31, 1958.

KANE, J.H. Boundary Element analysis in engineering continuun mechanics. Englewood Cliffs, N. J.: Prentice-Hall, 1994.

KARAMI, G.; ZARRINCHANG, J.; FOROUGHI, B. et al. An efficient analytical treatment of boundary integrals in direct Boundary Element analysis of plate bending problems. In: BREBBIA C.A.; DOMINGUES, J.; PARIS, F. eds. Boundary Elements XIV, v.2, 1992.

KARAN, V.J. Análise de flexão de placas pelo MEC incluindo não-linearidade física. Rio de Janeiro, 1992. Tese (Doutorado) - Universidade Federal do Rio de Janeiro, COPPE.

KARAN, V.J. Aplicação do Método dos Elementos de Contorno à Teoria de Reissner para flexão de placas. Rio de Janeiro, 1986. Dissertação (Mestrado) Universidade Federal do Rio de Janeiro, COPPE.

KATSIKADELIS, J.T.; ARMENAKAS, A.E. A new boundary equation solution to the plate problem. J. Appl. Mech. v.56(2), p.364-374, 1989.

KIRCHHOFF, G. Uber das gleichgewicht und die bewegung einer elastischen scleibe. J. Math., v.40, p.51-58, 1850.

KRAJCINOVIC, D.; FONSEKA, G.Y. The continuous damage theory of brittle materials. J. Appl. Mech., v.48, p. 816-824, December, 1981.

KUPRADZE, V.D. Potential methods in the theory of elasticity. Israel Progr. Sci. Transl., 1965.

LADEVÈSE, P.; PECASTAINGS, F. The optimal version of Reissner's theory. J. Appl. Mech. v.55(2), p.413-418, 1988.

LADEVÈZE, P. Sur une théorie de l'endomagement anisotrope, Int. Report n.34, laboratoire de Mécanique et technologie, Cachan, France, 1983.

LEVINSON, M. An accurate, simple theory of statics and dynamics of elastic plates. Mech. Res. Comm. v.7(6), p. 343-350, 1980.

MAZARS, J. Application de la mécanique de l'endommagement au comportement non lineaire et à la rupture du béton de structure. Thèse de Doctorat ès sciences, Université Paris 6, 1984.

MIKHLIN, S.G. Integral equations. London, Pergamon Press, 1957.

MINDLIN, R.D. Influence of rotatory inertia and shear on flexural motions of isotropic, elastic plates. J. Appl. Mech., v.13, p.31-38, 1951.

MOSHAIOV, A.; VORUS, W.S. Elasto-plastic bending analysis by a Boundary Element Method with initial plastic moments. Int. J. Solids Struct. v.22(11), p.1213-1229, 1986.

MUSKHELISHVILI, N. I. Some basic problems of the mathematical theory of elasticity. Noordhoff, Gronigen Holand, 1953.

NAYAK, G.C.; ZIENKIEVICZ, O.C. Elastoplastic stress analysis. A generalization for various constitutive relations including strain softening. Int. J. for Num. Meth. in Engng. v.5, p. 113-135, 1972.

NEVES, R.A. Cálculo de esforços e deslocamentos em estruturas de pisos de edifícios, considerando-se a influência das tensões cisalhantes. Dissertação (Mestrado). São Carlos, 2000. Escola de Engenharia de São Carlos, Universidade de São Paulo. 
NORDGREN, R.P. A bound on the error in plates theory. Quart. Appl. Math. V.28(4), p.587-595, 1971.

NORDGREN, R.P. A bound on the error in Reissner's theory of plates. Quart. Appl. Math. v.29, p. 551-556, 1972.

OLIVEIRA NETO, L. Uma formulação do Método dos Elementos de Contorno com três parâmetros nodais para placas delgadas e suas aplicações a problemas de engenharia estrutural. Tese (doutorado). São carlos, 1998. Escola de Engenharia de São Carlos, Universidade de São Paulo.

OLIVEIRA, A.L. Análise elastoplástica de placas de concreto armado pelo Método dos Elementos de Contorno utilizando a teoria de Reissner. Dissertação (Mestrado). Belo Horizonte, 1999. Escola de Engenharia da Universidade Federal de Minas Gerais.

ORTIZ, M. A constitutive theory for the inelastic behavior of concrete. Mech. Of Mat., v.4, p. 67-93, 1985.

ORTIZ, M. A continuum theory of inelasticity and failure of concrete under general states of stress. Brown University, 1985.

OWEN, D.R.J.; HINTON, E. Finite Element in Plasticity: Theory and Practice. Pineridge Press Limited. Swansea, U. K., 1980.

PAIVA, J.B. Formulação do Método dos Elementos de Contorno para flexão de placas e suas aplicações em engenharia de estruturas. São Carlos, 1987. Tese (Doutorado) - Escola de Engenharia de São Carlos, Universidade de São Paulo.

PALERMO JUNIOR, L. A análise de placas e o Método dos Elementos de Contorno. Campinas, 2000. Tese de Livre Docência - Universidade Estadual de Campinas.

PAPA, E. Sulla meccanica del danneggiamento con particolare riferimento alle murature. Tesi di Dottorato, Politecnico di Milano, 1990.

PEREGO, M. A. Danneggiamento dei materiali lapidei: leggi constitutive, analisi per elementi finiti ed applicazioni, 1989/90. Tesi de Laurea. Politecnico di Milano. Facoltà di Ingegneria. Dipartimento di ingegneria strutturale.

POPOVICS, S. A review of stress-strain relationships for concrete. ACI Journal. n.67-14, p.243-248, March, 1970.

PROENÇA, S.P.B. Notas sobre análise não-linear física de estruturas. Parte I: Teoria da plasticidade e técnicas numéricas. São Carlos, 1989. Escola de Engenharia de São Carlos, Universidade de São Paulo.

PROENÇA, S.P.B. Sobre modelos matemáticos do comportamento não-linear do concreto: análise crítica e contribuições. São Carlos, 1988. Tese (doutorado) Escola de Engenharia de São Carlos, Universidade de São Paulo.

RAO, P.S.; SUBRAHMANYAN, B.V. Trisegmental moment-curvature relations for reinforced concrete members. Proc. of the American Concrete Institute, n.70, p.346-351, May, 1973.

RASHID, V.R. Analysis of prestressed concrete pressure vessels, Nucl. Engng. Design, 7, 1968.

REISSNER, E. On a generalization of some formulas of the theory of moderately thich elastic plates. Int. J. Solids Struct., v.23(6), p.711-717, 1987.

REISSNER, E. On asymptotic Expansions for the sixth-order theory problem of transverse bending of orthotropic elastic plates. Comput. Meth. Appl. Mech. Engng., v.85(1), p.75-88, 1991. 
REISSNER, E. On bending of elastic plates. Quart. Appl. Math. v.5(1), p. 55-68, 1947.

REISSNER, E. On small deflections of sheardeformable elastic plates. Comput. Meth. Mech. Engng., v.59, p.227-233, 1986.

REISSNER, E. On the theory of bending of elastic plates. J. Math. Physics. v.23, p.184-191, 1944.

REISSNER, E. On the theory of transverse bending of elastic plates. Int. J. Solids Struct., v.12(8-A), p.545-554, 1976.

REISSNER, E. The effect of transverse shear deformation on the bending of elastic plates. J. Appl. Mech. v.12, p.A-69 - A-77.

RIBEIRO, G. O. Sobre a formulação do Método dos Elementos de Contorno para a flexão de placas usando as hipóteses de Reissner. São Carlos, 1992. Tese (Doutorado) - Escola de Engenharia de São Carlos, Universidade de São Paulo.

RIBEIRO, G.O.; VENTURINI, W.S. Aplicação do Método dos Elementos de Contorno ao cálculo de placas através da teoria de Reissner. MECOM-89. Anais do $\mathrm{X}$ Congresso Ibero-Latino-Americano sobre métodos computacionais em engenharia - II Encontro Nacional de Mecânica Computacional. Porto, Portugal, 1989.

RIBEIRO, G.O.; VENTURINI, W.S. Elastoplastic analysis of Reissner's plates using the Boundary Element Method. Ed. M.H. Aliabadi, Computational Mechanics Publications, London, 1998.

RIZZO, F.J. An integral approach to boundary value problems of classical elastostatics. Quart. Appl. Math.., v.25, n.1, p. 83-92, 1967.

ROCHA, F.S. Análise de descontinuidades pelo Método dos Elementos de Contorno. São Carlos, 1988. Tese (Doutorado) - Escola de Engenharia de São Carlos, Universidade de São Paulo.

RYCHTER, Z. A sixth-order plate theory - derivation and error estimates. J. Appl. Mech. v.54(2), p. 275-279, 1987.

RYCHTER, Z. An improved error estimate for Reissner's plate theory. Int. J. Solids Struct. V.24(5), p.537-544, 1988.

SALERNO, V.L.; GOLDBERG, M.A. Effect of shear deformations on the bending of retangular plates. J. Appl. Mech. p. 54-58, 1960.

SILVA, N.A. Aplicação do Método dos Elementos de Contorno à análise de placas sobre fundações elásticas. São Carlos, 1986. Dissertação (Mestrado) - Escola de Engenharia de São Carlos, Universidade de São Paulo.

SILVA, N.A. Aplicação do Método dos Elementos de Contorno às placas com enrijecedores utilizando a Teoria de Reissner. São Carlos, 1996. Tese (Doutorado)

- Escola de Engenharia de São Carlos, Universidade de São Paulo.

SOUTHWELL, R.V. Relaxation methods in theoretical physics. Oxford University Press. London, 1946.

STERN, M.A. A general boundary integral formulation for the numerical solution of plate bending problems. Int. J. Solids Struct. v.15, p.769-782, 1979.

SUIDAN, M.; SCHNOBRICH, W.C. Finite Element analysis of reinforced concrete. J. Struct. Div. ASCE, v. 99, p. 2109-2122. Oct., 1973.

TOTTENHAN, H. The Boundary Element Method for plates and shells, in: Banerjee, P.K.; Butterfield, R. eds. Developments in Boundary Element Methods -1. Applied Science Publ. London, p.173-205, 1979. 
TIMOSHENKO, S. P.: WOINOWSKY-KRIEGER, S. Theory of plates and shells. Mcgraw-hill Book Company. New York, N.Y. 1959.

TURNER, M.J. et al. Stiffnes and deflection analysis of complex structures. J. Aero. Science, v.23, p.805-823, 1956.

VABLE, M.; ZHANG,Y. A Boundary Element Method for plate bending problems. Int. J. Solids Struct., v.29, n.3, p.345-361, 1992.

VALLIAPPAN, S. Continuun mechanics fundamentals, Rotterdan. A. A. Balkema, 1981.

VENTURINI, W.S. Application of the boundary element formulation to solve geomechanical problems. Southampton, 1982. Thesis (Ph. D) - University of Southampton

WEEËN, F.V. Aplication of Boundary Integral Equation Method to Reissner's plate model. Int. J. Num. Meth. Engng., v.18(1), p.1-10, 1982.

WEEËN, F.V. Aplication of the Direct Boundary Element Method to Reissner's plate model. Boundary Element Method in Engineering, Procedings of the $4^{\text {th }}$. International Seminar. Southampton, 1982.

WESTPHAL JR., T.; BARCELLOS, C.S. Applications of the Boundary

Element Method to Reissner's and Mindlin's plate models; in: TANAKA, M.; BREBBIA, C.A.; HONMA, T., eds. Boundary Elements XII, 1989.

WU, B.C.; ALTIERO, N.J. A boundary integral method applied to plates of arbitrary plan form and arbitrary boundary conditions.. Computers \& Structures. v.10, p.703-707, 1979.

XIAO-YAN, L.; KUANG-MAO, H; XIUXI, W. Geometrically nonlinear analysis of a Reissner type plate by the Boundary Element Method. Computers \& Structures, v.37(6), p. 911-916, 1990.

ZIENKIEWICZ, O.C.; VALLIAPAN, S.; KING, J.P. Elasto-plastic solutions of engineering problems 'initial stress', Finite Element Approach. Int. J. Num. Meth. Engng. v.1, p.75-100, 1969. 


\section{APÊNDICE A SOLUÇÕES FUNDAMENTAIS}

$$
\begin{aligned}
& u_{\alpha \beta}^{*}=\frac{1}{8 \pi D(1-v)}\left\{[8 B-(1-v)(2 \ln z-1)] \delta_{\alpha \beta}-[8 A+2(1-v)] r_{, \alpha} r_{, \beta}\right\} \\
& u_{\alpha 3}^{*}=-u_{3 \alpha}^{*}=\frac{1}{8 \pi D}(2 \ln z-1) r r_{, \alpha} \\
& u_{33}^{*}=\frac{1}{8 \pi D(1-v) \lambda^{2}}\left[(1-v) z^{2}(\ln z-1)-8 \ln z\right]
\end{aligned}
$$

onde A e B são funções de $\mathrm{z}$ dadas por:

$$
\begin{aligned}
& A(z)=K_{0}(z)+\frac{2}{z}\left[K_{1}(z)-\frac{1}{z}\right] \\
& B(z)=K_{0}(z)+\frac{1}{z}\left[K_{1}(z)-\frac{1}{z}\right]
\end{aligned}
$$

sendo $\mathbf{K}_{\mathbf{0}}(\mathbf{z})$ e $\mathbf{K}_{\mathbf{1}}(\mathbf{z})$ funções de Bessel modificadas de ordem inteira, podendo ser calculadas através de expansões polinomiais dadas por ABRAMOWITZ E STEGUN (1965), incuídas no apêndice C. A expansão de A(z) para pequenos argumentos mostra que esta função é contínua, enquanto a expansão de $\mathbf{B}(\mathbf{z})$ apresenta singularidade do tipo $\ln \mathbf{z}$. $\mathbf{r}$ é a distância entre o ponto fonte $\xi$ e o ponto de deslocamento $\mathbf{x}$. 


$$
\begin{aligned}
& \mathbf{u}_{\alpha \beta, \gamma}^{*}=\frac{1}{4 \pi \mathrm{rD}(1-v)}\left[\left(4 \mathrm{~A}+4 \mathrm{zK}_{1}+1-\mathrm{v}\right) \mathrm{r}_{, \gamma} \delta_{\alpha \beta}-\right. \\
& \left.+2\left(2 \mathrm{zK}_{1}+8 \mathrm{~A}+1-\mathrm{v}\right) \mathrm{r}_{, \alpha} \mathbf{r}_{, \beta} \mathbf{r}_{, \gamma}+(4 \mathrm{~A}+1-\mathrm{v})\left(\mathbf{r}_{, \alpha} \delta_{\beta \gamma}+\mathbf{r}_{, \beta} \delta_{\alpha \beta}\right)\right] \\
& \mathbf{u}_{\alpha \beta, \beta}^{*}=-\frac{\mathbf{r}_{, \alpha}}{2 \pi \mathbf{r D}} \\
& u_{\alpha 3, \beta}^{*}=-u_{3 \alpha, \beta}^{*}=\frac{1}{8 \pi D}\left[\delta_{\alpha \beta}(2 \ln z-1)+2 r_{\alpha} r_{, \beta}\right] \\
& u_{\alpha 3, \alpha}^{*}=-u_{3 \alpha, \alpha}^{*}=\frac{\ln \mathrm{z}}{2 \pi \mathrm{D}} \\
& \mathbf{u}_{33, \alpha}^{*}=-\frac{r_{, \alpha}}{8 \pi r^{2} D(1-v)}\left[(1-v) z^{2}(2 \ln z-1)-8\right] \\
& p_{\alpha \beta}^{*}=-\frac{1}{4 \pi r}\left[\left(4 A+2 z K_{1}+1-v\right)\left(\delta_{\alpha \beta} r_{, n}+r_{, \beta} n_{\alpha}\right)+(4 A+1+v) r_{, \alpha} n_{\beta}-\right. \\
& \left.+2\left(8 \mathrm{~A}+2 \mathrm{zK}_{1}+1-v\right) \mathbf{r}_{, \alpha} \mathbf{r}_{, \beta} \mathbf{r}_{, \mathrm{n}}\right] \\
& \mathbf{p}_{\alpha 3}^{*}=\frac{\lambda^{2}}{2 \pi}\left(B \mathbf{n}_{\alpha}-A \mathbf{r}_{, \alpha} \mathbf{r}_{, n}\right) \\
& p_{3 \alpha}^{*}=-\frac{(1-v)}{8 \pi}\left[\left(2 \frac{(1+v)}{(1-v)} \ln z-1\right) n_{\alpha}+2 r_{, \alpha} r_{, n}\right] \\
& \mathbf{p}_{33}^{*}=-\frac{1}{2 \pi \mathbf{r}} \mathbf{r}_{, \mathbf{n}}
\end{aligned}
$$

onde $\mathbf{r}_{\mathbf{n}}$ é a derivada de $\mathbf{r}$ na direção normal no ponto $\mathbf{x}$.

$$
\begin{aligned}
& \mathbf{u}_{\alpha \beta \gamma}^{*}=\frac{1}{4 \pi r}\left[\left(4 \mathrm{~A}+2 \mathrm{zK} \mathrm{K}_{1}+1-\mathrm{v}\right)\left(\delta_{\beta \gamma} \mathbf{r}_{, \alpha}+\delta_{\alpha \gamma} \mathbf{r}_{, \beta}\right)+\right. \\
& \left.-\left(16 \mathrm{~A}+4 \mathrm{zK} \mathrm{K}_{1}+2-2 \mathrm{v}\right) \mathbf{r}_{, \alpha} \mathbf{r}_{, \beta} \mathbf{r}_{, \gamma}+(4 \mathrm{~A}+1+\mathrm{v}) \delta_{\alpha \beta} \mathbf{r}_{, \gamma}\right] \\
& \mathbf{u}_{\alpha \beta 3}^{*}=-\frac{(1-v)}{8 \pi}\left\{\left[2 \frac{(1+v)}{(1-v)} \ln \mathrm{z}-1\right] \delta_{\alpha \beta}+2 \mathbf{r}_{, \alpha} \mathbf{r}_{, \beta}\right. \\
& \mathbf{u}_{3 \beta \gamma}^{*}=\frac{\lambda^{2}}{2 \pi}\left(B \delta_{\gamma \beta}-\mathrm{Ar}_{, \gamma} \mathbf{r}_{, \beta}\right) \\
& \mathbf{u}_{3 \beta 3}^{*}=\frac{1}{2 \pi r} \mathbf{r}_{, \beta}
\end{aligned}
$$




$$
\begin{aligned}
& \mathbf{p}_{\alpha \beta \gamma}^{*}=\frac{D(1-v)}{4 \pi r^{2}}\left\{\left(4 A+2 z K_{1}+1-v\right)\left(\delta_{\alpha \gamma} n_{\beta}+\delta_{\gamma \beta} n_{\alpha}\right)+(4 A+1+3 v)\left(\delta_{\alpha \beta} n_{\gamma}\right)-\right. \\
& +\left(16 \mathrm{~A}+6 \mathrm{zK}_{1}+\mathbf{z}^{2} \mathbf{K}_{\mathrm{o}}+2-2 v\right)\left[\left(\mathbf{n}_{\alpha} \mathbf{r}_{, \beta}+\mathbf{n}_{\beta} \mathbf{r}_{, \alpha}\right) \mathbf{r}_{, \gamma}+\left(\delta_{\gamma \alpha} \mathbf{r}_{, \beta}+\delta_{\gamma \beta} \mathbf{r}_{, \alpha}\right) \mathbf{r}_{, \mathrm{n}}\right]- \\
& +\left(16 \mathrm{~A}+4 \mathrm{zK}_{1}+2+2 \mathrm{v}\right)\left(\delta_{\alpha \beta} \mathrm{r}_{, \gamma} \mathbf{r}_{, \mathrm{n}}+\mathbf{n}_{\gamma} \mathbf{r}_{, \alpha} \mathbf{r}_{, \beta}\right)+\left(96 \mathrm{~A}+32 \mathrm{zK}_{1}+4 \mathrm{z}^{2} \mathrm{~K}_{\mathrm{o}}+\right. \\
& \left.+8-8 v)) \mathbf{r}_{, \alpha} \mathbf{r}_{\beta} \mathbf{r}_{\gamma} \mathbf{r}_{, n}\right\} \\
& \mathbf{p}_{\alpha \beta 3}^{*}=\frac{\mathrm{D}(1-\mathrm{v}) \lambda^{2}}{4 \pi \mathbf{r}}\left[\left(2 \mathrm{~A}+\mathrm{zK}_{1}\right)\left(\mathbf{n}_{\alpha, \beta} \mathbf{r}_{, \beta}+\mathbf{n}_{\beta} \mathbf{r}_{, \alpha}\right)-\left(8 \mathrm{~A}+2 \mathrm{zK}_{1}\right)\right) \mathbf{r}_{, \alpha} \mathbf{r}_{, \beta} \mathbf{r}_{, \mathrm{n}}+ \\
& \left.+2 \mathrm{~A} \delta_{\alpha \beta} \mathrm{r}_{, \mathrm{n}}\right] \\
& \mathbf{p}_{3 \beta \gamma}^{*}=-\frac{D(1-v) \lambda^{2}}{4 \pi r}\left[\left(2 A+z K_{1}\right)\left(\delta_{\alpha \beta} r_{, n}+n_{\beta} r_{, \gamma}\right)+2 A n_{\gamma} r_{, \beta}-\left(8 A+2 z K_{1}\right)\right. \\
& ) \mathbf{r}_{, \gamma} \mathbf{r}_{, \beta} \mathbf{r}_{, \mathbf{n}}\right] \\
& \mathbf{p}_{3 \beta 3}^{*}=\frac{D(1-v) \lambda^{2}}{4 \pi r^{2}}\left[\left(z^{2} B+1\right) n_{\beta}-\left(z^{2} A+2\right) r_{\beta} r_{, n}\right] \\
& r_{\alpha \beta}^{*}=-\frac{(1-v)}{8 \pi}\left\{\delta_{\alpha \beta}\left[\frac{2(1+v)}{(1-v)} \ln z-1\right]+2 r_{, \alpha} r_{, \beta}+\right. \\
& \left.-\frac{4 v}{(1-v) \lambda^{2} \mathbf{r}^{2}}\left(2 \mathbf{r}_{, \alpha} \mathbf{r}_{\beta}-\delta_{\alpha \beta}\right)\right\} \\
& \mathbf{r}_{3 \beta}^{*}=\frac{\mathbf{r}_{, \beta}}{2 \pi \mathbf{r}} \\
& \mathbf{t}_{\alpha \beta \gamma}^{*}=\frac{1}{8 \pi \mathrm{r}}\left\{\left[8 \mathrm{~A}+4 \mathrm{zK} \mathrm{K}_{1}+2(1-v)\right]\left(\delta_{\alpha \gamma} \mathbf{r}_{, \beta}+\delta_{\beta \gamma} \mathbf{r}_{, \alpha}\right)+\right. \\
& \left.+2 r_{, \gamma} \delta_{\alpha \beta}(4 A+1+v)-2 r_{, \alpha} r_{, \beta} r_{, \gamma}\left[16 A+4 z K_{1}+2(1-v)\right]\right\} \\
& \mathbf{t}_{3 \beta \gamma}^{*}=\frac{\lambda^{2}}{2 \pi}\left(\mathbf{B} \delta_{\beta \gamma}-\mathbf{A r}_{, \beta} \mathbf{r}_{, \gamma}\right) \\
& \mathrm{v}_{\alpha, \beta}^{*}=\frac{\mathbf{r}^{2}}{128 \pi \mathrm{D}}\left[\delta_{\alpha \beta}(4 \ln \mathrm{z}-5)+2(4 \ln \mathrm{z}-3) \mathrm{r}_{, \alpha} \mathrm{r}_{, \beta}\right] \\
& v_{3, \beta}^{*}=\frac{r r_{, \beta}}{128 \pi D(1-v) \lambda^{2}}\left[32(2 \ln z-1)-z^{2}(1-v)(4 \ln z-5)\right]
\end{aligned}
$$




$$
\begin{aligned}
& w_{\alpha \beta}^{*}=-\frac{r}{64}\left\{( 4 \operatorname { l n } z - 3 ) \left[(1-v)\left(r_{, \beta} n_{\alpha}+r_{, \alpha} n_{\beta}\right)+(1-3 v) \delta_{\alpha \beta} r_{, n}+\right.\right. \\
& \left.+4\left[(1-v) \mathbf{r}_{, \alpha} \mathbf{r}_{, \beta}+v \delta_{\alpha \beta}\right] \mathbf{r}_{, \mathrm{n}}\right\}-\frac{v}{(1-v) \lambda^{2}} \mathbf{u}_{\alpha \beta \gamma}^{*} \mathbf{n}_{\gamma} \\
& \mathbf{w}_{3 \beta}^{*}=\frac{1}{8 \pi}\left[(2 \ln z-1) n_{\beta}+2 r_{, \beta} r_{, n}\right]-\frac{v}{(1-v) \lambda^{2}} u_{3 \beta \gamma}^{*} n_{\gamma} \\
& \lim _{\varepsilon \rightarrow 0} \int_{\Gamma_{\varepsilon}} t_{111}^{*}(\xi, x) d \bar{\Gamma}_{\varepsilon}(x)=\frac{1}{2 \pi}\left[(1-v) \operatorname{sen} \varphi+2(1+v)\left(\operatorname{sen} \varphi-\frac{\operatorname{sen}^{3} \varphi}{3}\right)\right] \\
& \lim _{\varepsilon \rightarrow 0} \int_{\Gamma_{\varepsilon}} t_{112}^{*}(\xi, x) d \bar{\Gamma}_{\varepsilon}(x)=\frac{1}{2 \pi}\left[(1-v) \cos \varphi-\frac{2}{3}(1+v) \cos ^{3} \varphi\right] \\
& \lim _{\varepsilon \rightarrow 0} \int_{\Gamma_{\varepsilon}} t_{121}^{*}(\xi, x) d \bar{\Gamma}_{\varepsilon}(x)=\frac{1}{2 \pi}\left[-(1-v) \cos \varphi-\frac{2}{3}(1+v) \cos ^{3} \varphi\right] \\
& \lim _{\varepsilon \rightarrow 0} \int_{\Gamma_{\varepsilon}} t_{122}^{*}(\xi, x) d \bar{\Gamma}_{\varepsilon}(x)=\frac{1}{2 \pi}\left[(1-v) \operatorname{sen} \varphi+\frac{2}{3}(1+v) \operatorname{sen}^{3} \varphi\right] \\
& \lim _{\varepsilon \rightarrow 0} \int_{\Gamma_{\varepsilon}} t_{221}^{*}(\xi, x) d \bar{\Gamma}_{\varepsilon}(x)=\frac{1}{2 \pi}\left[-(1-v) \operatorname{sen} \varphi+\frac{2}{3}(1+v) \operatorname{sen}^{3} \varphi\right] \\
& \lim _{\varepsilon \rightarrow 0} \int_{\Gamma_{\varepsilon}} t_{222}^{*}(\xi, x) d \bar{\Gamma}_{\varepsilon}(x)=\frac{1}{2 \pi}\left[-(1-v) \cos \varphi-2(1+v)\left(\cos \varphi-\frac{\cos ^{3} \varphi}{3}\right)\right] \\
& \mathrm{g}_{\alpha \beta \gamma \theta}^{*}(\xi)=\frac{1}{8}\left[(3-v)\left(\delta_{\alpha \gamma} \delta_{\theta \beta}+\delta_{\beta \gamma} \delta_{\theta \alpha}\right)-(1-3 v) \delta_{\alpha \beta} \delta_{\gamma \beta}\right] \\
& \mathbf{e}_{\alpha \beta \gamma \theta}^{*}=-\frac{1}{8 \pi r^{2}}\left\{\left[16 A+12 z K_{1}+2(1-v)+4 z^{2} K_{0}\right]\left(r_{, \theta} r_{, \beta} \delta_{\alpha \gamma}+r_{, \theta} r_{, \alpha} \delta_{\beta \gamma}\right)+\right. \\
& {\left[16 A+4 z K_{1}+2(1-v)\right]\left(2 \mathbf{r}_{, \beta} \mathbf{r}_{, \gamma} \delta_{\alpha \theta}+2 \mathbf{r}_{, \alpha} \mathbf{r}_{, \beta} \delta_{\gamma \theta}+2 \mathbf{r}_{, \gamma} \mathbf{r}_{, \theta} \delta_{\alpha \beta}+2 \mathbf{r}_{, \alpha} \mathbf{r}_{, \gamma} \delta_{\beta \theta}+\right.} \\
& \left.+\mathbf{r}_{, \alpha} \mathbf{r}_{, \theta} \delta_{\beta \gamma}+\mathbf{r}_{, \beta} \mathbf{r}_{, \theta} \delta_{\alpha \gamma}\right)-(4 A+1-v)\left(2 \delta_{\gamma \theta} \delta_{\alpha \beta}+\delta_{\alpha \theta} \delta_{\gamma \beta}+\delta_{\beta \theta} \delta_{\gamma \alpha}\right)- \\
& +\left(4 \mathrm{~A}+4 \mathrm{zK} \mathrm{K}_{1}+1-\mathrm{v}\right)\left(\delta_{\alpha \theta} \delta_{\beta \gamma}+\delta_{\alpha \gamma} \delta_{\beta \theta}\right)-\left[96 \mathrm{~A}+32 \mathrm{zK} \mathrm{K}_{1}+8(1-\mathrm{v})+\right. \\
& \left.\left.+4 z^{2} K_{0}\right]\left(2 r_{, \alpha} r_{, \beta} r_{, \gamma} r_{\theta}\right)+4 v \delta_{\alpha \beta}\left(2 r_{, \gamma} r_{, \theta}-\delta_{\gamma \theta}\right)\right\}
\end{aligned}
$$




$$
\begin{aligned}
& \mathbf{e}_{3 \beta \gamma \theta}^{*}=-\frac{\lambda^{2}}{2 \pi r}\left[\left(A+z K_{1}\right) r_{, \theta} \delta_{\beta \gamma}-\left(4 A+z K_{1}\right) r_{, \beta} \mathbf{r}_{, \gamma} \mathbf{r}_{, \theta}+\right. \\
& \left.+\mathbf{A}\left(\mathbf{r}_{, \gamma} \delta_{\beta \theta}+\mathbf{r}_{, \beta} \delta_{\gamma \theta}\right)\right] \\
& I_{\alpha \beta}^{*}=-\frac{R}{48 \pi}\left\{4 r_{, \alpha} r_{, \beta} r_{, n}\left(1-v-\frac{12 v}{z^{2}}\right)+\left(2 r_{, n} \delta_{\alpha \beta}+r_{, \alpha} n_{\beta}+r_{, \beta} n_{\alpha}\right)\right. \\
& \left.\left[(1-v)\left(2 \ln z-\frac{5}{3}\right)+\frac{12 v}{z^{2}}\right]+6 v r_{, n} \delta_{\alpha \beta}\left[2 \ln z-1+\frac{4 v}{(1-v) z^{2}}\right]\right\}- \\
& \left(A_{1} \cos \theta+B_{1} \operatorname{sen} \theta\right) \frac{R^{2}}{64 \pi}\left\{2 r_{, \alpha} r_{, \beta} r_{, n}\left[(1-v)\left(2 \ln z+\frac{1}{2}\right)-\frac{8 v}{z^{2}}\right]+\right. \\
& \left(2 r_{, n} \delta_{\alpha \beta}+r_{, \alpha} n_{\beta}+r_{, \beta} n_{\alpha}\right)\left[(1-v)\left(2 \ln z+\frac{3}{2}\right)+\frac{8 v}{z^{2}}\right]+ \\
& \left.8 v r_{, n} \delta_{\alpha \beta}\left[2 \ln z-1+\frac{4 v}{(1-v) z^{2}}\right]\right\} \\
& I_{3 \beta}^{*}=-\frac{1}{48 \pi}\left\{r_{, \beta} r_{, n}\left[z^{2} \frac{(1-v)}{2}\left(\ln z-\frac{13}{12}\right)+6(v+2)\right]-\right. \\
& 3 n_{, \beta}\left[z^{2} \frac{(1-v)}{4}\left(\ln z-\frac{5}{4}\right)+2(v-2)\left(\ln z-\frac{1}{2}\right)\right\}+ \\
& \left(A_{1} \cos \theta+B_{1} \operatorname{sen} \theta\right) \frac{R}{64 \pi}\left\{r _ { , \beta } r _ { , n } \left[z^{2} \frac{(1-v)}{50}(29-20 \ln z)-\frac{16}{3}(v-2)\left(\ln z-\frac{1}{3}\right)+\right.\right. \\
& \left.\left.\frac{8}{3}(v+4)\right]-4 n_{\beta}\left[\frac{(1-v)}{5} z^{2}\left(\ln z-\frac{6}{5}\right)+\frac{4}{3}(v-2)\left(\ln z-\frac{1}{3}\right)\right]\right\}
\end{aligned}
$$




\section{APÊNDICE B}

\section{FUNDAMENTOS MATEMÁTICOS}

\section{B.1. INTRODUÇÃO}

Este apêndice é destinado à apresentação de alguns conceitos matemáticos que facilitarão o entendimento dos capítulos que compõem o presente trabalho, principalmente àqueles que não estão muito familiarizados com o tema, evitando-se assim, consultas à bibliografias complementares.

\section{B.2. NOTAÇÃO INDICIAL}

A notação indicial é uma forma compacta ou abreviada usada convenientemente para a manipulação de grupos de símbolos.

Considere-se a equação algébrica seguinte:

$$
\mathbf{a}_{1} \mathbf{x}_{1}+\mathbf{a}_{2} \mathbf{x}_{2}+\mathbf{a}_{3} \mathbf{x}_{3}+\mathbf{a}_{4} \mathbf{x}_{4}=b
$$

Esta mesma equação pode ser escrita usando-se o símbolo do somatório, como segue: 


$$
\sum_{i=1}^{4} a_{i} x_{i}=b
$$

Seja o sistema de 3 equações a 4 incógnitas $\mathrm{x}_{\mathrm{j}}$ :

$$
\begin{aligned}
& a_{11} x_{1}+a_{12} x_{2}+a_{13} x_{3}+a_{14} x_{4}=b_{1} \\
& a_{21} x_{1}+a_{22} x_{2}+a_{23} x_{3}+a_{24} x_{4}=b_{2} \\
& a_{31} x_{1}+a_{32} x_{2}+a_{33} x_{3}+a_{34} x_{4}=b_{3} \\
& a_{41} x_{1}+a_{42} x_{2}+a_{43} x_{3}+a_{44} x_{4}=b_{4}
\end{aligned}
$$

O mesmo sistema pode ser representado por:

$$
a_{i 1} x_{1}+a_{i 2} x_{2}+a_{i 3} x_{3}+a_{i 4} x_{4}=b_{i} \quad(i=1,2,3)
$$

ou ainda:

$$
\sum_{j=1}^{4} a_{i j} x_{j}=b_{i} \quad(i=1,2,3)
$$

Observe-se que há um índice repetido, j, do lado esquerdo de (b.2.b), que é somado no intervalo de 1 a 4. Há também um índice i, que não é repetido e que, portanto, não está envolvido no processo do somatório. Isto significa que durante o processo do somatório envolvendo o índice $\mathbf{j}$, o índice $\mathbf{i}$ permanece o mesmo em cada termo da soma. O índice $\mathbf{j}$ é chamado índice mudo e o índice $\mathbf{i}$, índice livre.

\section{b.2.1. Convenção de somatório}

A convenção de somatório estabelece a soma sem o uso explícito do símbolo. Se um índice ocorre duas vezes em um termo, está implícito que este índice deve ser somado para todos os valores no intervalo deste índice.

Assim, a expressão (b.2.b) pode ser escrita da seguinte forma: 


$$
\mathbf{a}_{\mathrm{ij}} \mathbf{x}_{\mathbf{j}}=\mathbf{b}_{\mathbf{i}}
$$

tomando-se o intervalo dos índices do contexto do problema em análise.

\section{b.2.2. Delta de Kronecker}

O uso do Delta de Kronecker em determinadas equações possibilita uma forma de expressar variáveis que existem apenas sob certas combinações. A sua definição é:

$$
\delta_{i j}= \begin{cases}1 ; & \text { se } \mathbf{i}=\mathbf{j} \\ 0 ; & \text { se } \mathbf{i} \neq \mathbf{j}\end{cases}
$$

\section{b.2.3. Permutações}

Para os problemas da Engenharia, os índices relacionados às coordenadas cartesianas assumirão valores 1,2 ou 3 nos problemas tri-dimensionais e 1 ou 2 nos problemas bi-dimensionais.

Assim, no primeiro caso a notação:

$\mathbf{u}_{\mathbf{i}}$ indica 3 variáveis;

$\mathbf{U}_{\mathbf{i j}}$ indica 9 variáveis;

$\mathbf{D}_{\mathbf{i j k}}$ indica 27 variáveis;

$\delta_{\text {ijkl }}$ indica 81 variáveis.

\section{b.2.4. Diferenciação}

A notação de diferenciação numa determinada direção é caracterizada por uma vírgula antes do índice. Os exemplos a seguir são esclarecedores.

$$
\mathbf{f}_{, \mathbf{i}}=\frac{\partial \mathbf{f}}{\partial \mathbf{x}_{\mathbf{i}}}
$$




$$
\begin{aligned}
& \mathbf{U}_{\mathbf{i j}, \mathbf{j}}=\frac{\partial \mathbf{U}_{\mathrm{ij}}}{\partial \mathbf{x}_{\mathbf{j}}}=\frac{\partial \mathbf{U}_{11}}{\partial \mathbf{x} 1}+\frac{\partial \mathbf{U}_{12}}{\partial \mathbf{x} 2}+\frac{\partial \mathbf{U}_{13}}{\partial \mathbf{x} 3} \quad(\mathrm{p} / \mathrm{i}=1) \\
& =\frac{\partial \mathbf{U}_{21}}{\partial \mathbf{x} 1}+\frac{\partial \mathbf{U}_{22}}{\partial \mathbf{x} 2}+\frac{\partial \mathbf{U}_{23}}{\partial \mathbf{x} 3} \quad(\mathrm{p} / \mathrm{i}=2) \\
& =\frac{\partial \mathbf{U}_{31}}{\partial \mathbf{x} 1}+\frac{\partial \mathrm{U}_{32}}{\partial \mathbf{x} 2}+\frac{\partial \mathbf{U}_{33}}{\partial \times 3}
\end{aligned}
$$

\section{B.3. OPERADOR DIFERENCIAL $\nabla$ (GRADIENTE)}

Seja $\mathbf{f}$ um campo escalar de sorte que $\mathbf{f}=\mathbf{f}(\mathbf{x}, \mathbf{y}, \mathbf{z})$, definida num certo domínio do espaço. Se existem as derivadas primeiras de $\mathbf{f}$ nesse domínio, então elas formam as componentes do vetor gradiente de f. Assim:

$$
\nabla \mathbf{f}=\frac{\partial \mathbf{f}}{\partial \mathbf{x}} \mathbf{i}+\frac{\partial \mathbf{f}}{\partial \mathbf{y}} \mathbf{j}+\frac{\partial \mathbf{f}}{\partial \mathbf{z}} \mathbf{k}
$$

\section{B.4. DIVERGENTE DE UM VETOR ( $\nabla . v)$}

Sejam as componentes de um campo vetorial $\mathbf{v}$ dadas por $\mathbf{v}_{\mathbf{x}}, \mathbf{v}_{\mathbf{y}}$ e $\mathbf{v}_{\mathbf{z}}$. Se estas componentes possuírem derivadas parciais primeiras num domínio $\mathbf{D}$, então o escalar $\operatorname{div} \mathbf{v}$ é dado por:

$$
\operatorname{div} \mathbf{v}=\nabla \cdot \mathbf{v}=\frac{\partial \mathbf{v}_{x}}{\partial \mathbf{x}}+\frac{\partial \mathbf{v}_{\mathbf{y}}}{\partial y}+\frac{\partial \mathbf{v}_{z}}{\partial z}
$$

\section{B.5. OPERADOR LAPLACIANO $\nabla^{2}$}

Outro operador diferencial muito utilizado é o operador laplaciano $\nabla^{2}$, assim definido:

$$
\nabla^{2} f=\nabla \cdot(\nabla \mathbf{f}) \frac{\partial^{2} f}{\partial x^{2}}+\frac{\partial^{2} f}{\partial y^{2}}+\frac{\partial^{2} f}{\partial z^{2}}
$$




\section{B.6. JACOBIANO}

O Jacobiano é utilizado para mudar as variáveis de integração ou diferenciação de um sistema para outro. Seja, então, transformar a variável $\mathbf{x}$, na integral a seguir, na variável $\zeta$ :

$$
\int_{x 1}^{\mathrm{x} 2} \mathbf{f}(\mathbf{x}) \mathrm{dx}=\int_{\zeta 1}^{\zeta 2} \mathbf{f}[\mathbf{x}(\zeta)] \mathbf{J}(\zeta) \mathrm{d} \zeta
$$

Neste caso, o Jacobiano $\mathbf{J}(\zeta)$ é definido por:

$$
\mathbf{J}(\zeta)=\frac{\partial \mathbf{x}(\zeta)}{\partial \zeta}
$$

No caso de integrais duplas, a mudança das variáveis é dada por:

$$
\int_{y 1}^{y 2} \int_{x 1}^{x 2} f(x, y) d x d y=\int_{v 1}^{v 2} \int_{u 1}^{u 2} f(x(u, v), y(u, v)) J(u, v) d u d v
$$

onde o Jacobiano é assim definido:

$$
J(u, v)=\frac{\partial(x, y)}{\partial(u, v)}=\left|\begin{array}{ll}
\frac{\partial x}{\partial u} & \frac{\partial x}{\partial v} \\
\frac{\partial y}{\partial u} & \frac{\partial y}{\partial v}
\end{array}\right|=\frac{\partial x}{\partial u} \frac{\partial y}{\partial v}-\frac{\partial y}{\partial u} \frac{\partial x}{\partial v}
$$

\section{B.7. TRANSFORMAÇÃO DE TENSÃO DE CAUCHY}

Considere-se um corpo deformável em equilíbrio, sujeito à ação de um sistema de forças e com as devidas condições de apoio. Para tal caso qualquer pedaço do objeto também estará em equilíbrio. Considere-se ainda, o tetraedro removido deste corpo, como indicado na figura b.1. 


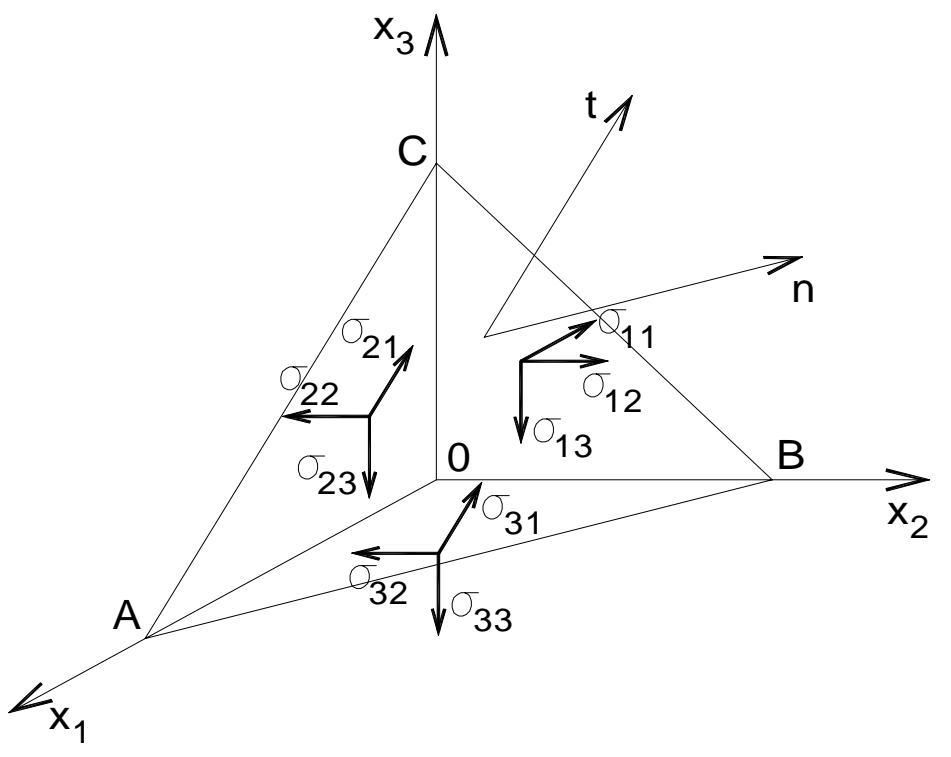

FIGURA B.1. Equilíbrio de um tetraedro

Sejam:

$\sigma_{11}, \sigma_{12}, \sigma_{13}$ as componentes de tensão sobre o plano $\mathbf{O B C}$;

$\boldsymbol{\sigma}_{22}, \boldsymbol{\sigma}_{21}, \boldsymbol{\sigma}_{23}$ as componentes de tensão sobre o plano $\mathbf{O A C}$;

$\sigma_{33}, \sigma_{31}, \sigma_{32}$ as componentes de tensão sobre o plano $\mathbf{O A B}$;

$\mathbf{t}_{1}, \mathbf{t}_{\mathbf{2}}, \mathbf{t}_{3} \quad$ as componentes de tensão sobre o plano de normal $\mathbf{n}$.

A transformação de tensão de Cauchy estabelece que as componentes do vetor de tensão sobre um plano com normal $\mathbf{n}$ estão relacionadas com as componentes sobre os planos $\mathbf{O B C}, \mathbf{O A C}$ e $\mathbf{O A B}$ da seguinte forma:

$$
\begin{aligned}
& \mathbf{t}_{1}=\sigma_{11} \mathbf{n}_{1}+\sigma_{12} \mathbf{n}_{2}+\sigma_{13} \mathbf{n}_{3} \\
& \mathbf{t}_{2}=\sigma_{21} \mathbf{n}_{1}+\sigma_{22} \mathbf{n}_{2}+\sigma_{23} \mathbf{n}_{3} \\
& \mathbf{t}_{3}=\sigma_{31} \mathbf{n}_{1}+\sigma_{32} n_{2}+\sigma_{33} n_{3}
\end{aligned}
$$

ou, em notação indicial:

$$
\mathbf{t}_{\mathbf{i}}=\sigma_{\mathrm{ij}} \mathbf{n}_{\mathbf{j}}
$$




\section{B.8. FUNÇÃO DELTA DE DIRAC}

Considere-se a função $\mathbf{F}(\mathbf{x}, \mathbf{d}, \mathbf{a})$ mostrada na fig. b.2, de tal forma que sua integral seja igual à unidade sobre o domínio onde a função existe.

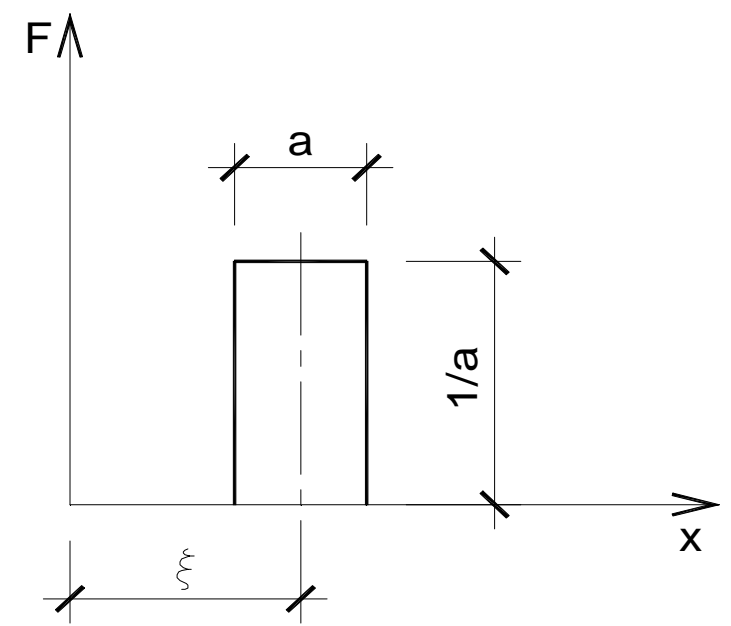

FIGURA B.2. Definição da função Delta de Dirak. Função F(x,d,a).

Então:

$$
F(x, d, a)=\left\{\begin{array}{lc}
\frac{1}{a} & \text { para } d-\frac{a}{2} \leq x \leq d+\frac{a}{2} \\
0 & \text { nos outros casos }
\end{array}\right.
$$

A função Delta é definida como o limite da função $\mathbf{F}(\mathbf{x}, \mathbf{d}, \mathbf{a})$, quando a tende a zero:

$$
\delta(\mathbf{x}-\mathbf{d})=\lim _{\mathbf{a} \rightarrow \mathbf{0}} \mathrm{F}(\mathbf{x}, \mathbf{d}, \mathbf{a})
$$

Isto significa que quando a região de domínio da função torna-se menor, o valor da função aumenta de tal forma que a resultante (efeito integrado) da função permanece constante. 
A propriedade seletiva da função Delta envolve a integral do produto da função Delta por outra função g. A fim de obter-se esta propriedade, considere-se a integração do produto de $\mathbf{g}$ pela função Delta, no limite quando a largura a torna-se extremamente pequena.

$$
I(d, a)=\int_{-\infty}^{+\infty} g(x) F(x, d, a) d x=\int_{d-a / 2}^{d+a / 2} g(x) F(x, d, a) d x
$$

O Teorema do Valor Médio do cálculo integral estabelece que o valor de uma integral definida sobre um dado intervalo é igual ao valor do integrando avaliado em algum ponto $\boldsymbol{\alpha}$ dentro daquele intervalo vezes a largura do intervalo. No limite, quando a tende a zero, $\boldsymbol{\alpha}$ deve assumir o valor $\mathbf{d}$. Então:

$$
\lim _{\mathbf{a} \rightarrow 0} I(d, a)=\left\{g(\alpha) \frac{1}{a}\right\} a=g(d)=\int_{-\infty}^{+\infty} g(x) \delta(x-d) d x
$$

Assim, a função Delta quando envolvida em um processo de integração com outra função, seleciona o valor da outra função no ponto onde a função Delta existe.

\section{B.9. VALOR PRINCIPAL DE CAUCHY}

Considere-se a integral de uma função $\phi(t)$, sendo $t$ um ponto de um contorno $\Gamma$, não definida num ponto $\mathbf{t}_{\mathbf{0}}$. Para a definição de tal integral, altera-se o trecho $\Gamma$ para $\Gamma-\bar{\Gamma}$, onde $\bar{\Gamma}$ é um segmento da curva $\Gamma$, determinado por uma circunferência de raio $\boldsymbol{\varepsilon}$ e com centro em $\mathbf{t}_{\mathbf{0}}$ (fig. b.3). 


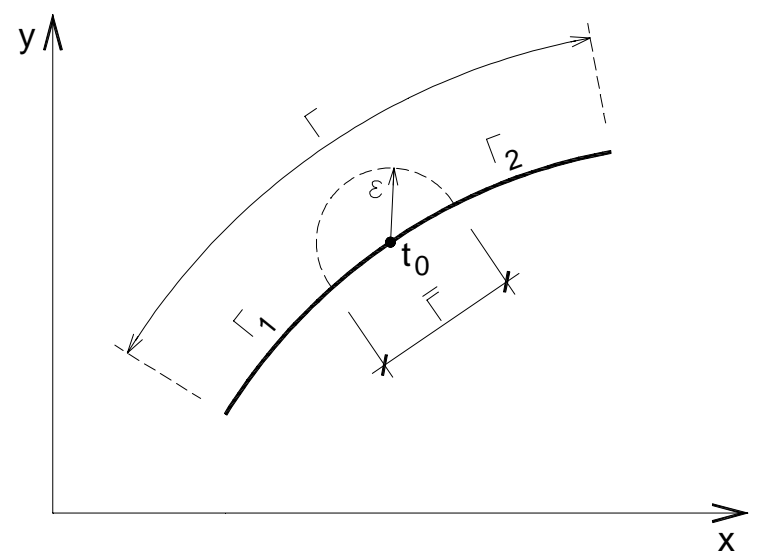

FIGURA B.3. Definição de uma integral de contorno. (ROCHA, 1988)

Assim, diz-se que a integral

$$
\int_{\Gamma} \phi(\mathbf{t}) \mathbf{d} \Gamma
$$

existe, quando existir o limite

$$
\lim _{\varepsilon \rightarrow 0} \int_{\Gamma-\Gamma} \phi(t) d \Gamma
$$

denominado 'integral singular' da função $\phi(t)$ ao longo do contorno $\Gamma$.

Considerando-se que o ponto $\mathrm{t}_{0}$ não esteja contido nas extremidades do segmento da curva $\Gamma$, o trecho $\Gamma-\bar{\Gamma}$ fica dividido em dois trechos $\Gamma_{1}$ e $\Gamma_{2}$. Logo, o limite dado pela eq.(b.20) torna-se:

$$
\lim _{\varepsilon \rightarrow 0} \int_{\Gamma_{1}} \phi(t) d \Gamma+\lim _{\varepsilon \rightarrow 0} \int_{\Gamma_{2}} \phi(t) d \Gamma
$$


Em existindo o limite de cada uma das integrais expressas na eq.(b.21), diz da integral dada na eq.(b.19) não apresentar 'especial singularidade' e o seu limite pode ser calculado normalmente. Por outro lado, se aqueles limites isoladamente não existirem, usa-se o seguinte recurso:

$$
\lim _{\varepsilon \rightarrow 0}\left(\int_{\Gamma_{1}} \phi(t) d \Gamma+\int_{\Gamma_{2}} \phi(t) d \Gamma\right)
$$

Se os termos integrais da eq.(b.22) se cancelarem, antes de se fazer $\boldsymbol{\varepsilon}$ tender a zero, o limite dado pela eq.(b.22) existe e, consequentemente, a integral dada na eq.(b.19). Aos limites dados em (b.21) e (b.22) denominam-se valor principal de Cauchy, da integral dada na eq.(b.19).

Com isso pode-se escrever:

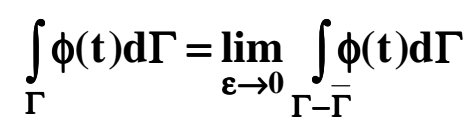

Caso a integral dada em (b.19) não apresente especial singularidade, o ponto $\mathbf{t}_{\mathbf{0}}$ pode pertencer a uma das extremidades do segmento da curva.

Obviamente, a existência dos limites (b.21) e (b.22) depende do tipo de função $\phi(t)$ e do tipo de curva $\Gamma$.

\section{B.10. CONDIÇÃO DE HÖLDER}

Considere-se a fig. b.4, onde se define um segmento de curva pelos pontos $\mathbf{t}_{\mathbf{1}}$ e $\mathbf{t}_{\mathbf{2}}$, de comprimento $\mathbf{S}_{\mathbf{2}}-\mathbf{s}_{\mathbf{1}}$. Os parâmetros $\mathbf{s}_{\mathbf{1}}$ e $\mathbf{s}_{\mathbf{2}}$ são coordenadas curvilíneas.

Uma função $\phi(\mathbf{t})$, definida e contínua nos pontos dessa curva, satisfaz a condição de Hölder quando:

$$
\left|\phi\left(\mathbf{t}_{2}\right)-\phi\left(\mathbf{t}_{1}\right)\right| \leq \mathbf{A}\left|\mathbf{t}_{2}-\mathbf{t}_{1}\right|^{\mu}
$$




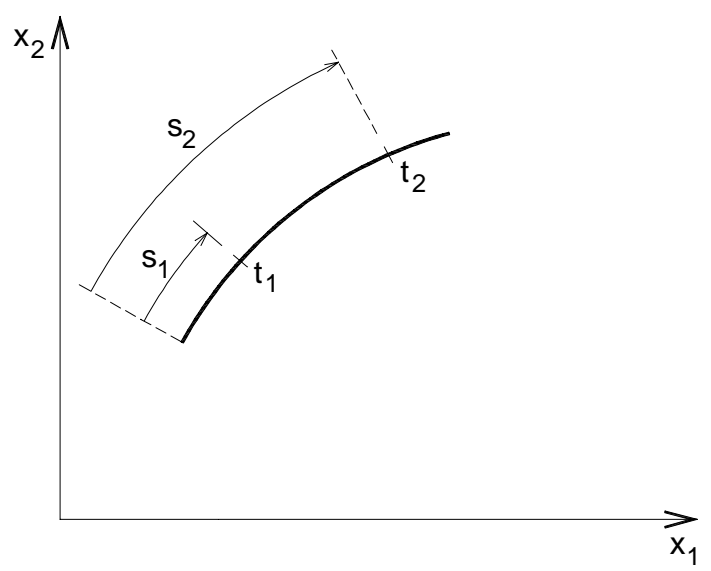

FIGURA B.4. curva genérica para definição da condição de Hölder (ROCHA, 1988).

onde A e $\boldsymbol{\mu}$ são constantes positivas, denominadas constante de Hölder e índice de Hölder, respectivamente.

Da condição de Hölder, conclui-se que o valor absoluto da diferença entre os valores da função $\phi$ em dois pontos da curva é menor ou igual a A pela potência do valor absoluto da distância entre esses pontos elevado a $\mu$. As constantes $\mathbf{A}$ e $\boldsymbol{\mu}$ devem ser, para cada caso, convenientemente escolhidas. Ressalta-se que a função $\phi$, cuja variável dependente caracteriza a posição de um ponto genérico da curva, em geral, não é a representação analítica da curva.

O índice de Hölder $\boldsymbol{\mu}$, para problemas usuais, não é maior que um. Assim, sempre será suposto que $\mathbf{0}<\boldsymbol{\mu} \leq \mathbf{1}$ (ROCHA, 1988). 


\section{APÊNDICE C}

\section{FUNÇÕES DE BESSEL MODIFICADAS}

As funções de Bessel modificadas de ordem inteira $\mathbf{k}_{\mathbf{0}}(\mathbf{z})$ e $\mathbf{k}_{\mathbf{1}}(\mathbf{z})$ podem ser calculadas pelas expansões polinomiais seguintes, dadas por ABRAMOWITZ (1965), sendo $\mathbf{z}$ um argumento real:

a) para $0<\mathrm{z} \leq 2$ :

$$
\begin{aligned}
& k_{o}(z)=-\ln \left(\frac{z}{2}\right)_{0}(z)-0,57721566+0,4227842\left(\frac{z}{2}\right)^{2}+0,23069756\left(\frac{z}{2}\right)^{4}+ \\
& +0,0348859\left(\frac{z}{2}\right)^{6}+0,00262698\left(\frac{z}{2}\right)^{8}+0,0001075\left(\frac{z}{2}\right)^{10}+0,0000074\left(\frac{z}{2}\right)^{12}+ \\
& +\varepsilon \\
& \mid \varepsilon<10^{-8}
\end{aligned}
$$




$$
\begin{aligned}
& k_{1}(z)=\frac{1}{z}\left[z \ln \left(\frac{z}{2}\right) I_{1}(z)+1+0,15443144\left(\frac{z}{2}\right)^{2}-0,67278579\left(\frac{z}{2}\right)^{4}+\right. \\
& -0,18156897\left(\frac{z}{2}\right)^{6}-0,01919402\left(\frac{z}{2}\right)^{8}-0,00110404\left(\frac{z}{2}\right)^{10}+ \\
& \left.-0,00004686\left(\frac{z}{2}\right)^{12}+\varepsilon\right] \\
& \mid \varepsilon<8.10^{-8}
\end{aligned}
$$

sendo:

$$
\begin{aligned}
& I_{0}(z)=1+3,5156229 t^{2}+3,0899424 t^{4}+1,2067492 t^{6}+ \\
& +0,2659732 t^{8}+0,0360768 t^{10}+0,0045813 t^{12}+\varepsilon \\
& |\varepsilon|<1,6.10^{-7} \\
& I_{1}(z)=z\left[0,5+0,87890594 t^{2}+0,51498869 t^{4}+0,15084934 t^{6}+\right. \\
& \left.+0,02658733 t^{8}+0,00301532 t^{10}+0,00032411 t^{12}+\varepsilon\right] \\
& \mid \varepsilon<8.10^{-9}
\end{aligned}
$$

com:

$$
\mathrm{t}=\frac{\mathrm{z}}{3,75}
$$


b) para $z \geq 2$ :

$$
\begin{aligned}
& k_{0}(z)=\frac{1}{\sqrt{z} \mathrm{e}^{\mathrm{z}}}\left[1,25331414-0.07832358\left(\frac{2}{\mathrm{z}}\right)+0,02189568\left(\frac{2}{\mathrm{z}}\right)^{2}+\right. \\
& -0,01062446\left(\frac{2}{\mathrm{z}}\right)^{3}+0,00587872\left(\frac{2}{\mathrm{z}}\right)^{4}-0,0025154\left(\frac{2}{\mathrm{z}}\right)^{5}+0,00053208\left(\frac{2}{\mathrm{z}}\right)^{6}+ \\
& +\varepsilon] \\
& |\varepsilon|<1,9.10^{-7}
\end{aligned}
$$

$$
\begin{aligned}
& k_{1}(z)=\frac{1}{\sqrt{z} \mathrm{e}^{\mathrm{z}}}\left[1,25331414+0,23498619\left(\frac{2}{\mathrm{z}}\right)-0,0365562\left(\frac{2}{\mathrm{z}}\right)^{2}+\right. \\
& +0,01504268\left(\frac{2}{\mathrm{z}}\right)^{3}-0,00780353\left(\frac{2}{\mathrm{z}}\right)^{4}+0,00325614\left(\frac{2}{\mathrm{z}}\right)^{5}-0,00068245\left(\frac{2}{\mathrm{z}}\right)^{6} \\
& +\varepsilon] \\
& |\varepsilon|<2,2.10^{-7}
\end{aligned}
$$

Definem-se ainda:

$$
A(z)=k_{0}(z)+\frac{2}{z}\left[k_{1}(z)-\frac{1}{z}\right] ; \quad B(z)=k_{0}(z)+\frac{1}{z}\left[k_{1}(z)-\frac{1}{z}\right]
$$

Derivando-se as expressões (c.8) e rearranjando-se os termos, obtém-se:

$$
\begin{aligned}
& A^{\prime}(z)=-k_{1}(z)-\frac{2}{z}\left[k_{0}(z)+\frac{2}{z} k_{1}(z)-\frac{2}{z^{2}}\right] \\
& B^{\prime}(z)=-k_{1}(z)-\frac{1}{z}\left[k_{0}(z)+\frac{2}{z} k_{1}(z)-\frac{2}{z^{2}}\right]
\end{aligned}
$$

Substituindo-se a primeira das equações (c.8) nas eqs.(c.9), vem:

$$
A^{\prime}(z)=-\frac{1}{z}\left[z k_{1}(z)+2 A(z)\right] ; \quad B^{\prime}(z)=-\frac{1}{z}\left[z k_{1}(z)+2 A(z)\right]
$$




\section{APÊNDICE D \\ FLUXOGRAMA DO PROGRAMA PRINCIPAL}

\section{D.1. Diagrama N-S}

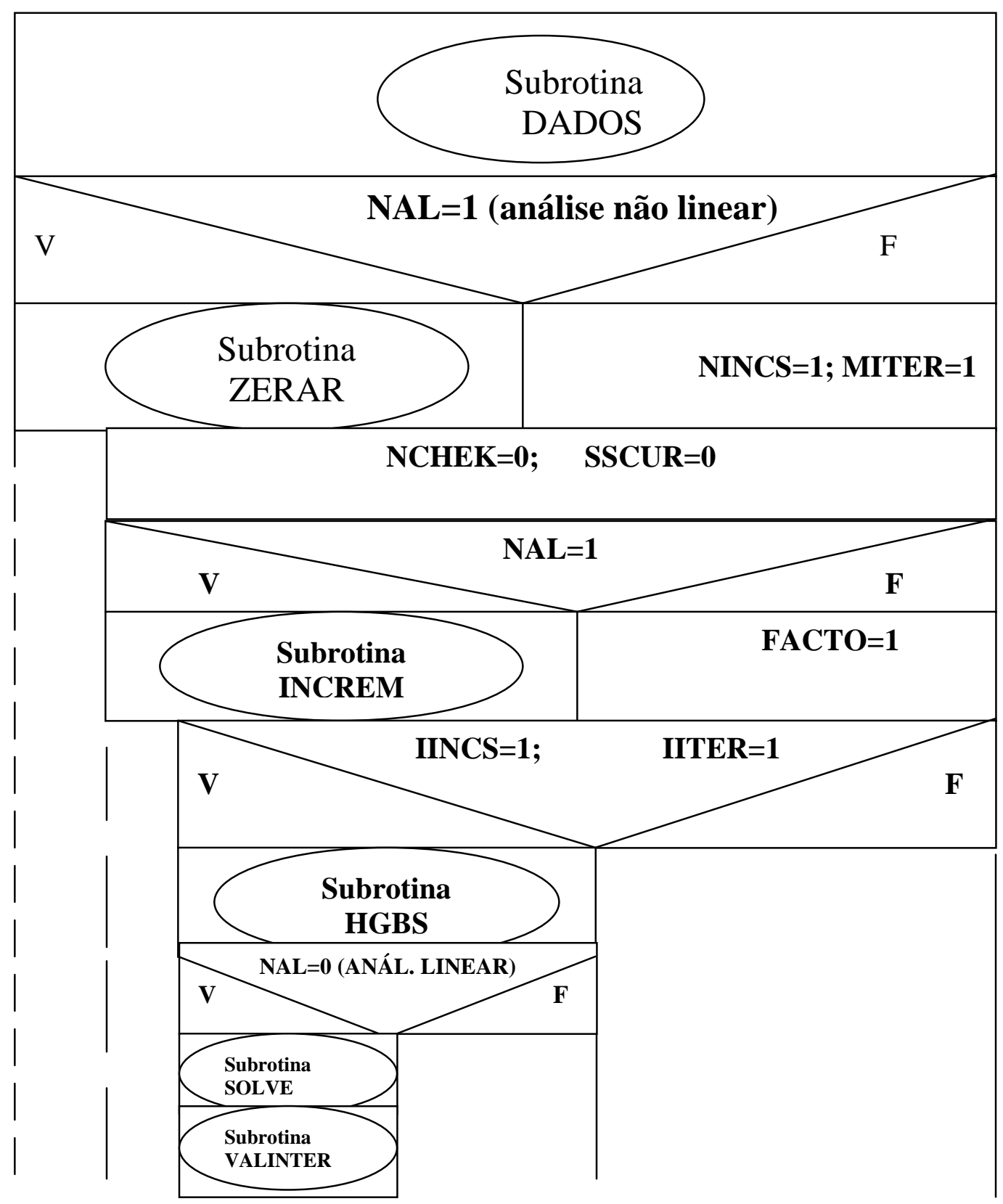




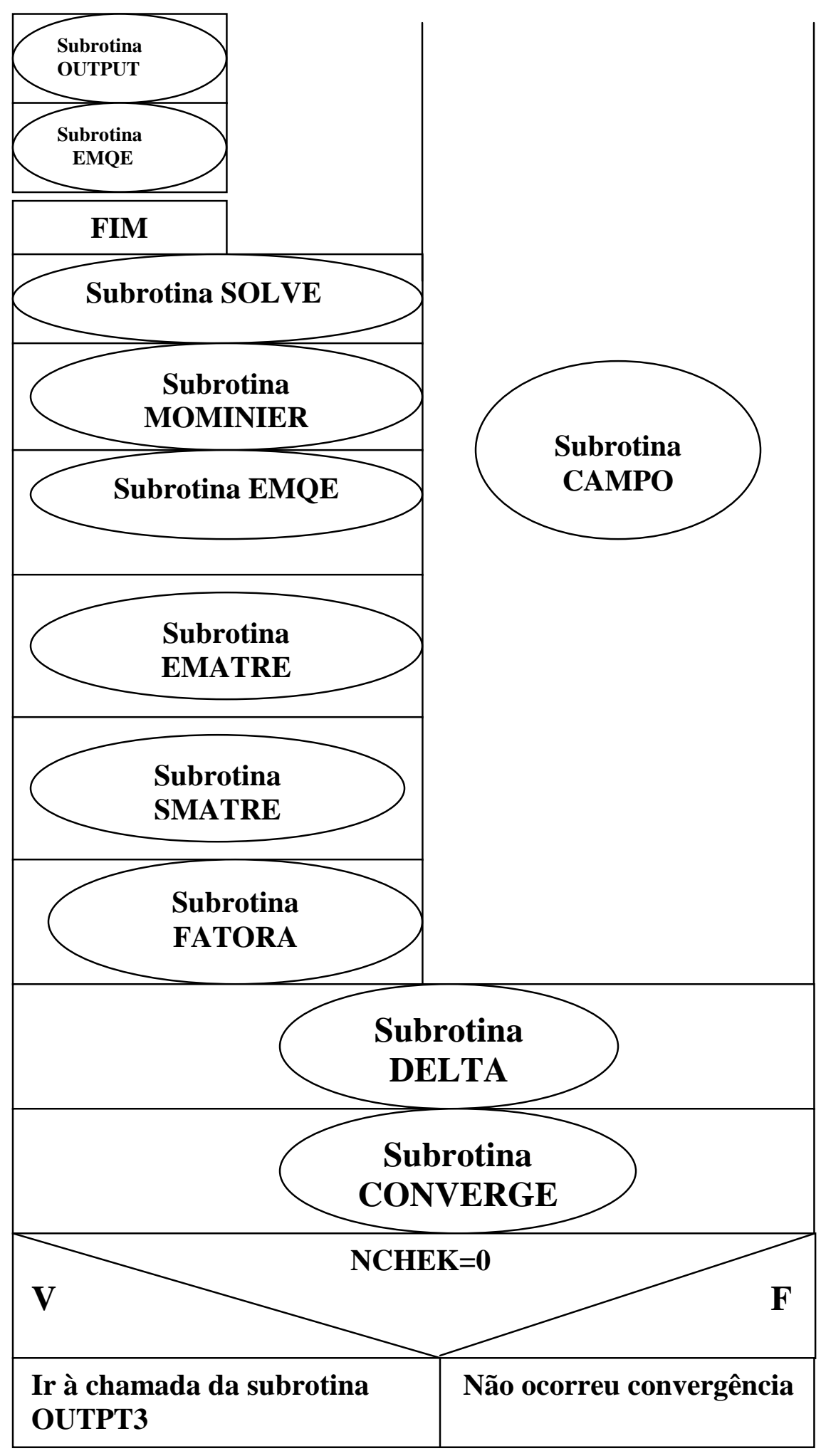

Repetir até IITER = MITER 


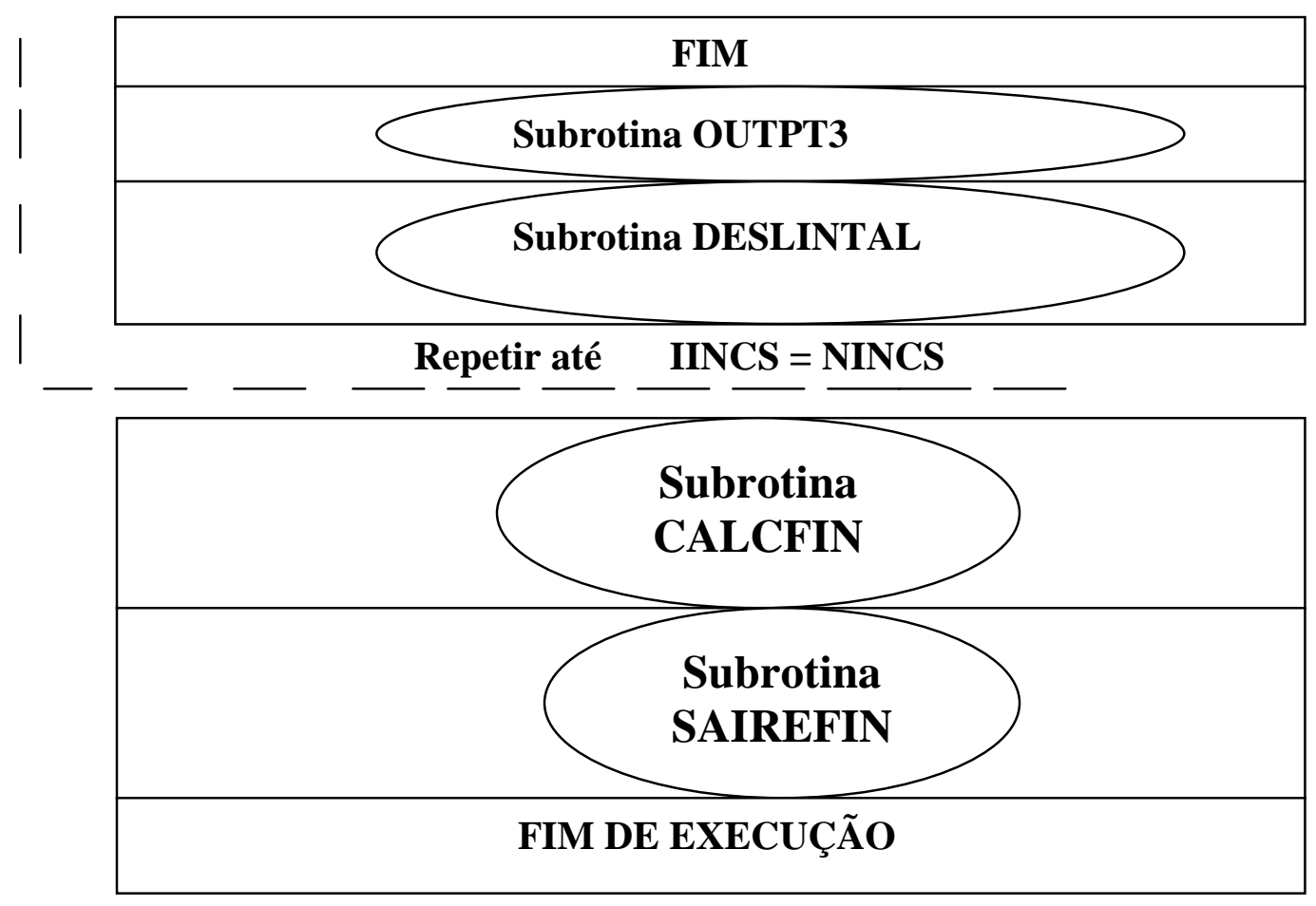

\section{D.2. Variáveis}

NAL: Variável que caracteriza o tipo de análise $(\mathrm{NAL}=0$ análise linear; $\mathrm{NAL}=1$ análise não linear);

NINCS: Número total de incrementos;

MITER: Número máximo de iterações;

NCHEK; SSCUR: : Variáveis que verificam a convergência do processo incremental iterativo;

FACTO: Fator que especifica a magnitude do incremento de carga;

IINCS; IITER: Incremento e iteração em curso, respectivamente;

\section{D.3. Subrotinas:}

ZERAR: atribui valor zero às variáveis pertinentes;

INCREM: leitura de dados dos incrementos de carga e tolerância para o esforço normal;

HGBS: Montagem das matrizes H G B S;

SOLVE: Resolve o sistema de equações; 
VALINTER: cálculo dos valores internos (deslocamentos e reações);

OUTPUT: Saída de resultados;

EMQE: Cálculo dos esforços nos pontos internos;

MOMINIER: Cálculo da matriz cujos coeficientes representam a influência do campo de momentos iniciais nos valores de contorno;

EMATRE: Formação da matriz $\mathbf{S}$ (início) cujos coeficientes representam a influência do campo de momentos iniciais nos valores dos esforços nos pontos internos;

SMATRE: Formação da matriz $\mathbf{S}$ (término);

FATORA: Cálculo dos esforços fatorados;

CAMPO: Cálculo dos momentos em cada incremento considerando-se os momentos residuais;

DELTA: Cálculo dos momentos residuais;

CONVERGE: Verifica a convergência do processo incremental iterativo;

OUTPT3: Saída de resultados após a convergência em cada incremento;

DESLINTAL: Cálculo do deslocamento total ao final de cada incremento;

CALCFIN: Cálculo dos esforços finais devidos à carga total aplicada;

SAIREFIN: Saída dos resultados (deslocamentos internos e reações) finais. 